\title{
APPLICATION OF AN INVERSE MODEL IN THE COMMUNITY MODELING EFFORT RESULTS
}

by

\author{
Huai-Min Zhang \\ M.S. in Physical Oceanography, MIT/WHOI Joint Program, 1991 \\ Submitted in partial fulfillment of the \\ requirements for the degree of \\ DOCTOR OF PHILOSOPHY \\ at the \\ MASSACHUSETTS INSTITUTE OF TECHNOLOGY \\ and the \\ WOODS HOLE OCEANOGRAPHIC INSTITUTION
}

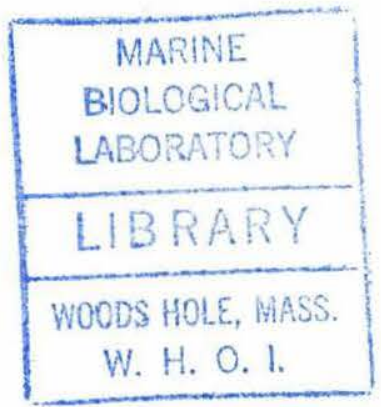

February 1995

(C) Huai-Min Zhang 1994

The author hereby grants to MIT and to WHOI permission to reproduce and to distribute copies of this thesis document in whole or in part.

Signature of Author

Joint Program in Physical Oceanography Massachusetts Institute of Technology

Woods Hole Oceanographic Institution

December 14, 1994

Certified by

Nelson G. Hogg

$\Omega_{1}, 1$ Thesis/Supervisor

Accepted by . .

Carl Wunsch

Chairman, Joint Committee for Physical Oceanography Massachusetts Institute of Technology Woods Hole Oceanographic Institution 


\title{
APPLICATION OF AN INVERSE MODEL IN THE COMMUNITY MODELING EFFORT RESULTS
}

\author{
by \\ Huai-Min Zhang \\ Submitted in partial fulfillment of the requirements for the degree of \\ Doctor of Philosophy at the Massachusetts Institute of Technology \\ and the Woods Hole Oceanographic Institution
}

Dec. 12, 1994

\begin{abstract}
Inverse modeling activities in oceanography have recently been intensified, aided by the oncoming observational data stream of WOCE and the advance of computer power. However, interpretations of inverse model results from climatological hydrographic data are far from simple. This thesis examines the behavior of an inverse model in the WOCE CME (Community Modeling Effort) results where the physics and the parameter values are known. The ultimate hypotheses to be tested are whether the inferred circulations from a climatological hydrographic data set (where limited time means and spatial smoothing are usually used) represent the climatological ocean general circulations, and what the inferred "diffusion" coefficients really are.
\end{abstract}

The inverse model is first tested in a non-eddy resolving numerical GCM ocean. Numerical/scale analyses are used to test whether the inverse model properly represents the GCM ocean. Experiments show how biased answers could result from an incorrect model, and how a correct model must produce the right answers.

When the inverse model is applied to the time-mean hydrographic data of an eddy-resolving GCM ocean in the fine grid resolution of the GCM, the estimated horizontal circulation is statistically consistent with the EGCM time means in both patterns and values. Although the flow patterns are similar, the uncertainties for the GCM time means and the inverse model estimates are different. The former are very large, such that the GCM time-mean circulation has no significance in the deep ocean. The latter are much smaller, and with them the estimated circulations are well defined. This is consistent with the concept that ocean motions are very energetic, while variations of tracers (temperature, salinity) are low frequency. The inverse model succeeded in extracting the ocean general circulation from the "climatological" hydrographic data.

The estimated vertical velocities are also statistically indistinguishable from the GCM time means. However, significant differences between the estimated "dif- 
fusion" coefficients and the EGCM eddy diffusion coefficients are found at certain locations. These discrepancies are attributed to the differences in physics of the inverse model and the EGCM ocean. The "diffusion" coefficients from the inversion parameterize not only the eddy fluxes, but also (part of) the temporal variation and biharmonic terms which are not explicitly included in the inverse model.

Given the essentially red spectrum of the ocean, it makes sense to look for smooth solutions. Aliasing due to subsampling on a coarse grid and the effects of spatial smoothing are addressed in the last part of this thesis. It is shown that this aliasing could be greatly reduced by spatial smoothing. The estimated horizontal circulation from the spatially smoothed time-mean EGCM hydrographic data with a coarse grid resolution $\left(2.4^{\circ}\right.$ longitude by $2.0^{\circ}$ latitude) is generally consistent with the spatially smoothed EGCM time means. Significant differences only occur at some grid points at great depths, where the GCM circulations are very weak.

The conclusions of this study are different from some previous studies. These discrepancies are explained in the concluding chapter.

Finally, it should be pointed out that the issue of properly representing a $\mathrm{GCM}$ ocean by an inverse model is not identical to the issue of representing the real ocean by the same inverse model, since the GCM ocean is not identical to the real ocean. Numerical calculations show that both the non-eddy resolving and the eddy-resolving GCM oceans used in this work are evolving towards a statistical equilibrium. In the real ocean, the importance of temporal variation terms in the property conservation equations should also be analyzed when a steady inverse model is applied to a limited time-mean (the climatological) data set.

Thesis Supervisor:

Dr. Nelson G. Hogg

Senior Scientist

Woods Hole Oceanographic Institution 


\section{Table of Contents}

$\begin{array}{ll}\text { Abstract } & 3\end{array}$

1. INTRODUCTION 9

1.1 INVERSE MODELING IN PHYSICAL OCEANOGRAPHY $\ldots . \quad 9$

1.2 EXAMINATION OF INVERSE MODELS . . . . . . . . . 15

1.3 THE APPROACH OF THIS WORK . . . . . . . . . . . . . 22

2. THE MODELS 28

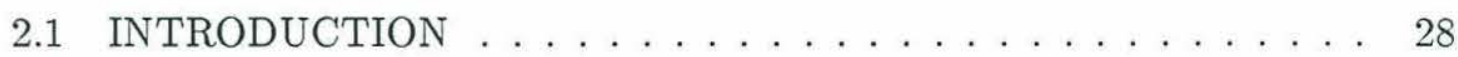

2.2 THE NUMERICAL OCEAN GENERAL CIRCULATION MODEL 29

2.3 THE INVERSE MODEL $\ldots \ldots \ldots \ldots \ldots \ldots$

2.3 .1 Introduction $\ldots \ldots \ldots \ldots \ldots \ldots \ldots \ldots$

2.3.2 Formulation of the Equations . . . . . . . . . . . 35

2.3.3 Surface Layer Model . . . . . . . . . . . . . . . . . . . . . . 38

2.3.4 Finite Difference Formulation . . . . . . . . . . . . 40

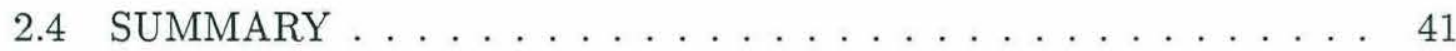

3. APPLICATION OF THE INVERSE MODEL IN A NON-EDDY RESOLVING NUMERICAL GCM OCEAN 44

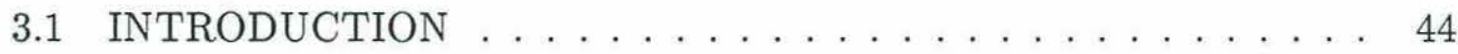

3.2 ACCURACY OF THE INVERSE MODEL ASSUMPTIONS IN THE NON-EDDY RESOLVING GCM OCEAN . . . . . . . . . . . . 46

3.2 .1 Dynamic Equation . . . . . . . . . . . . . . . 46

3.2.2 Conservation Equation for Water Properties . . . . . . . . . 50

3.3 INVERSE MODEL RESULTS $\ldots \ldots \ldots \ldots \ldots \ldots \ldots$ 
3.3 .1 Introduction $\ldots \ldots \ldots \ldots \ldots \ldots \ldots$

3.3.2 Upper-Layer Model Results Without Surface Frictional Layer

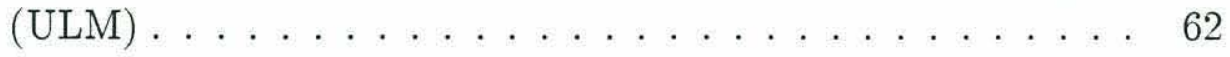

3.3.3 Experiments on the Ekman Pumping Velocity Constraint . . 69

3.3.4 Model with Frictional Surface Layer-Determine the Air-Sea Fluxes . . . . . . . . . . . . . . . . . 83

3.3.5 Deep-Layer Model (DLM) . . . . . . . . . . . . . . . . . . . 91

3.3.6 A Full-Layer Model—Can We Make Consistent Estimates? . 93

3.4 EXPERIMENTS ON THE PARAMETERIZATION . . . . . . . 110

3.4 .1 Introduction . . . . . . . . . . . . . . 110

3.4.2 A,K,w and Air-Sea Fluxes as $3^{\text {rd }}$ Order Polynomials-PAR I 112

3.4.3 $A, K$ and Air-Sea Fluxes as $3^{\text {rd }}$ Order Polynomials while $w$ is a Point-wise Unknown-PAR II . . . . . . . . . . . . . . 120

3.4.4 Discussion . . . . . . . . . . . . . . . . 125

3.5 SUMMARY AND CONCLUDING REMARKS . . . . . . . . . . 126

\section{APPLICATION OF THE INVERSE MODEL IN AN EDDY-RESOLVING} NUMERICAL GCM OCEAN 132

4.1 INTRODUCTION . . . . . . . . . . . . . . . . . . . . . 132

4.2 THE NUMERICAL GCM OCEAN . . . . . . . . . . . . . . . 135

4.3 INVERSION OF THE TIME-MEAN FIELDS . . . . . . . . . . 145

4.3 .1 Introduction $\ldots \ldots \ldots \ldots \ldots \ldots$

4.3.2 Accuracy of the Inverse Model in the Time Mean Fields of the EGCM Ocean . . . . . . . . . . . . . . . 147

4.3.3 Inverse Model Results of the Time-Mean Fields . . . . . . . 153

4.3.4 Summary and Discussion . . . . . . . . . . . 177 
4.4 APPLICATION OF THE INVERSE MODEL IN THE SPATIALLY SMOOTHED TIME-MEAN FIELDS OF THE EDDY-RESOLVING GCM OCEAN . . . . . . . . . . . . . . . . . 180

4.4 .1 Introduction . . . . . . . . . . . . . . . 180

4.4.2 Subsampling Aliasing - the Accuracy of the Inverse Model in a Coarse-grid Resolution . . . . . . . . . . . . . . . . 182

4.4.3 Inverse Model Results in the Spatially Smoothed Time-Mean Ocean . . . . . . . . . . . . . . . . . 196

4.4.4 Sensitivity of the Inverse Model Results on the Vertical Level (Layer) Number . . . . . . . . . . . . . . . . 218

4.4.5 Summary and Discussion . . . . . . . . . . . . 221

5. CONCLUSION AND DISCUSSION

225

APPENDIX A: SPATIAL SMOOTHING AND FINITE-DIFFERENCE GRADIENT

246

$\begin{array}{ll}\text { Acknowledgments } & 253\end{array}$

$\begin{array}{ll}\text { References } & 255\end{array}$ 


\section{Chapter 1. INTRODUCTION}

This thesis extensively examines one of the many inverse models used in the field of physical oceanography in the context of the WOCE CME results (Community Modeling Effort of the World Ocean Circulation Experiment). This chapter describes the motivations, background, and methodology as well as the organization of this thesis.

\subsection{INVERSE MODELING IN PHYSICAL OCEANOG- RAPHY}

Inverse methods have been widely used in many fields of science and engineering. As one example, the use of inverse methods in medical imaging has recently been reviewed by Louis (1992). Their use in geophysics was described by, for example, Backus and Gilbert (1967), and was summarized in a textbook by Menke (1984). The introduction and systematic study of inverse methods in physical oceanography has been carried out by Wunsch (e.g., Wunsch, 1977, 1978, 1984, 1988a, 1994) and other physical oceanographers (e.g., Bennett, 1992).

Inverse models in physical oceanography address of the inadequacies of traditional methods (the descriptive method and the dynamic method) for determining the ocean circulations. One of the major goals of physical oceanography is to describe the large-scale time-mean circulation in the world oceans. Determining the general ocean circulation is an important step toward understanding the global climate system (e.g., the global budget of heat, fresh water, $\mathrm{CO}_{2}$, etc.), the distri- 
bution of water properties (temperature, salinity, etc.) and chemical tracers as well as biological nutrients and sediment movement in the oceans.

Unfortunately, compared to atmospheric observations, direct measurements of the circulation in the world oceans are much more difficult and costly. Typically available are the in situ measurements of hydrographic data and chemical tracers from individual cruises. The two traditional means to deduce the oceanic circulation from these available information are the so called descriptive method and dynamic method. The descriptive method uses the spatial distribution of water properties to "draw" the directions of the water movement qualitatively (e.g., Wüst's, 1935, core-layer analysis; Montgomery's, 1938, isentropic analysis). No quantitative computations can be made from these methods. In addition, as the water property distributions involve both advective and diffusive processes, the "arrows" drawn along a water "tongue" can be incorrectly interpreted. Examples of similar property fields resulting from different physical processes were found in Zhang and Hogg's (1992) inversion in the Brazil Basin. Although coincidence of flows along temperature (and salinity) tongues on the isopycnals was found in one region, cases of flows along isotherms were also found in other regions.

The dynamic method utilizes the density field of observations to calculate the vertical shear of the horizontal velocities through the geostrophic and hydrostatic balances (the thermal wind relation). Both theory (e.g., Pedlosky, 1987) and observations (e.g., Bryden, 1977) show that the large scale oceanic circulations are mainly in geostrophic balance, although there are violations of this assumption in some regions of the ocean (e.g., in the boundary layers). But there is a difficult issue to deal with in this method. Intrinsically the dynamic method can only give us the vertical shears or differences of the lateral velocities between two 
surfaces. To determine the absolute velocities in the water column (assuming the geostrophic assumption is valid), the absolute velocity at a certain depth must also be prescribed. This traditionally involves the so-called "level-of-no-motion" or the velocity at the "reference level". Although various ways to determine the "level of no motion" have been proposed (e.g., the ocean bottom or at certain deep depth, or an interface of two different water masses which appear to be flowing in opposite directions), there are neither theoretical, nor observational justification for the existence of a level of no motion. Small errors in the reference velocities can produce large errors in calculating the heat and other water property transports across a basin scale hydrographic section.

The determination of the "reference level" velocity stimulated the growth of inverse models in physical oceanography (Wunsch, 1977, 1978; Stommel and Schott, 1977; Schott and Stommel, 1978). The velocities calculated from the dynamic method alone (from the thermal wind relation) do not ensure flows consistent with the distribution of tracer fields. Inverse models sought to remove these inconsistencies by requiring the circulation to simultaneously satisfy a variety of constraints deduced from the observed property distributions. Multiple conservation equations to determine water transports were also used in earlier work by Hidaka (1940), Riley (1951) and Wright (1969).

Since the pioneering work of Wunsch (1978) and Schott and Stommel (1978), a variety of inverse models have been developed with differing degrees of complexity applied mainly in the North Atlantic (due to the relatively dense coverage of hydrographic sections in this ocean basin), and a few of other basins. Wunsch (1980) described the problem of combining hydrography with marine geodesy and satellite altimetry for the purpose of determining the general circulation of the oceans, defin- 
ing the eddy field, and improving the marine geoid. In early box inverse models, it appears that the problem is underdetermined, leading to a range of circulation patterns compatible with geostrophic balance and the conservation of mass (e.g., Wunsch and Grant, 1982). In this case one usually gets an infinite set of solutions instead of getting the solution. Wunsch (1984) argued that the range of possible oceanic solutions could be narrowed by adding to the inversion information derived from other data sets (direct velocity or tracer measurements). Using an eclectic model, Wunsch (1984) succeed in putting useful bounds on the meridional flux of heat in the North Atlantic ocean. Olbers et al. (1985) applied the beta-spiral method to the North Atlantic data and, in addition to the determination of the absolute flow field, showed that the method could be used to infer diffusion rates. The method appeared to work well in areas where diffusion was a dominant process in the tracer balance, but the results were less compelling where this was not the case. In Joyce et al.'s (1986) work, data from a shipborne acoustic profiling device have been combined with hydrographic sections across the Gulf Stream and are used to estimate the absolute flow fields. The inverse results for the Gulf Stream transports are plausibly close to previous calculations. Inversions were also done individually on the Doppler data and the hydrographic data. They concluded that the inversion of the combined data sets produces results much improved over those using either acoustic or hydrographic constraints in isolation.

The more complicated inverse models are designed to infer not only the absolute velocities, but also the mixing rates in the ocean. Mixing has been proven to be an important process in water property balances, especially in the deep ocean where advection is weak. Using simple models, Tziperman (1987) also showed the importance of mixing processes in driving the deep thermohaline circulation and for 
determining the basic vertical density stratification of the wind driven circulation. Knowledge of mixing rates are also important for (forward) numerical ocean modeling, such as the CME, as experiments have shown that GCM results are sensitive to the specific parameterization of the eddy diffusivity (Bryan, 1987). However, in practice the eddy diffusion coefficients from the inversions are very sensitive to data noise (e.g., Olbers, 1989). Oxygen, nutrients, tritium, radiocarbon and other tracers have all been used to constrain the inverse model solutions for these parameters (Wunsch, 1984; Olbers et al, 1985; Hogg, 1987; Jenkins, 1987; Schlitzer, 1987; Spitzer et al, 1989). Their usefulness is usually determined by adequacy of knowledge of the measurement errors (e.g., instrumental and sampling problems) and of the sources and sinks for the tracers. The use of transient tracers for determining ocean transport is a more difficult problem. A first step towards making inference from sparse transient tracer information was taken by Wunsch (Wunsch, 1988a). Later he treated this problem using control theory (Wunsch, 1988b). These approaches are reviewed, together with other inverse problems and techniques, in a lecture in the NATO-Advanced Study Institute (Wunsch, 1989). A later application in the North Atlantic ocean was carried out by Mémery and Wunsch (1990).

Development of inverse models in oceanography has taken more complicated forms. An example of the use of linear programming methods in the context of oceanographic tracer models in studying nutrient and carbon cycles in the North Atlantic is shown in Schlitzer (1989). Linear programming appears to be a powerful tool to examine the whole range of possible solutions. The method provides diagnostics to identify how well the model parameters are determined and which parts of the data provide important/redundant information. It is often possible to determine what additional information is needed to improve the solutions for cer- 
tain parameters. Taking account the errors associated with each data set explicitly (by allowing the density field to be adjusted by the inverse model), Mercier (1986, 1989, 1993) formulated nonlinear inverse problems. Nonlinear optimization was also used by Wunsch more recently in the North Atlantic (Wunsch, 1994). Methods based on adjoint equations are not new but have recently attracted much interest as they become feasible to large systems. They are particularly appropriate for time-dependent systems with a large numbers of variables (e.g., initial or boundary conditions) for which optimum values are sought, and are widely used in data assimilation. Examples are Tziperman et al (1989), Marotzke (1992), Marotzke and Wunsch (1993), Schlitzer (1993), Schiller and Willebrand (1994), and Schiller (1994).

The recent increase of inverse model applications in oceanography is linked to the availability of more accurate data to constrain the parameter solutions. In the early stages of inverse modeling in physical oceanography, extensively used were the "box" inverse models due to the fact that the observations in the ocean were rarely adequate to compute the gradients needed in the "finite difference" models. WOCE provides the opportunity to utilize the finite difference inverse models more efficiently in the ocean (one of the recent examples was Martel and Wunsch, 1993a,b). In fact, in addition to the observations, ocean modeling is another goal of WOCE. The two key modeling objectives of WOCE are to develop ocean models for predicting climate change and to develop methods for analyzing the WOCE field data. The analyzed WOCE data sets will be used to initialize and test models and to study long term changes in the ocean circulation. (WCRP, WOCE Report No. $112,1994)$. 
In WOCE, temperature and salinity fields are measured to give information on the large scale baroclinic (vertically varying) velocity field. Surface drifters and other floats are being released to obtain direct information about the absolute velocity field. Satellites are being used to measure the wind stress on the ocean and also the ocean surface topography (giving the surface geostrophic velocity field). The data sets anticipated from WOCE will come close to defining the physical and chemical state of the ocean. A combination of in situ observations of hydrography and chemistry with altimetry, windfields (from both scatterometry and conventional analyses), float trajectories (e.g., Owens, 1991), current meter measurements, and direct estimates of water mass fluxes across various straits and sills (e.g., Bryden et al, 1994) ought to vastly reduce the existing uncertainty over the state of the ocean circulation. This will result in a global dataset of unprecedented scope. Given the oncoming data stream, an important issue is how to use these data to understand the climate state of the ocean and its physics (e.g., the general circulations, mixing rates, etc.).

\subsection{EXAMINATION OF INVERSE MODELS}

Although inverse models are widely used in physical oceanography, the interpretation of the inverse model results is far from simple, and the issue of the "validation" of the inverse models, i.e. whether the inverse model results represent the real ocean, is still not resolved. There are two aspects in this issue: first, the

physics of the inverse model are not the same as those of the ocean; and secondly, inverse model solutions are not unique. The inverse model physics are usually much simpler than the ocean physics. Also, instead of getting the solution from an in- 
verse model, one usually get $a$ solution based on one's own criteria. For example, a box inverse model usually results in an underdetermined system, and the parameter norm, or the kinetic energy at the reference level, is minimized in solving the equations (e.g., Wunsch, 1978). Some finite difference inverse models result in overdetermined systems (e.g., Hogg, 1987), and the residual norm of the equations is minimized to obtain the parameter solutions. In forward modeling, there are also subjective factors. First, different numerical models have different physics, and they are simplified versions of the physics in the real ocean. Secondly, there are various parameters which must be subjectively chosen. For instance, atmosphere forcing (wind stress, heat flux, etc) has large statistical uncertainties. Any values within the statistical errors are valid and there is no significant difference among them. In this sense, the forward modeling results are also not unique.

The essential question here is to what extent the inverse model results resemble reality. For the same reason that inverse models in physical oceanography were developed, the lack of direct observations of the circulations in the oceans makes it difficult to test the inverse models in the real ocean. Direct measurements of velocity in the ocean are rare, and one may doubt the representativeness of the comparisons of the measurements with inverse model results. One can argue that even if the comparisons show consistency at the very few measurement "spots", in terms of large scale ocean circulation, it still may not be consistent in the vast unmeasured area; and vice versa. Also, current meter measurements are usually taken over a very limited time period, while the estimated circulations by inverse models from hydrographic data are intended to be climatological. Reid et al (1977) reported deep/abyssal current meter measurements whose daily means have large variations within two months in the vicinity of the Vema Channel, although the measurements 
are quite steady within the channel. More recent example are Schmitz and Hogg (1983), Tarbell et al. (1994). The strong eddy fields make the extraction of the weak ocean general circulation difficult.

A natural choice for testing the inverse models is to apply them in (forward) numerical model results. Fiadeiro and Veronis (1984) tested two inverse models in a highly idealized channel model. With the advance in computer power, more and more sophisticated (forward) numerical models have been/are being developed, with the aim to simulate the physical processes in the real ocean. Testing the inverse models in these numerical oceans is an important step toward understanding the functioning of the inverse models and the interpretation of the inverse model results.

Historically the numerical general circulation models (GCMs) for the ocean come in two varieties. On one hand are models with active thermodynamics and moderate to high vertical resolution, but low horizontal resolution (the Non-eddy Resolving Models or the so-called OGCMs - the Ocean General Circulation Models). These have been developed in an attempt to represent the large-scale hydrographic structure and climatic properties (water mass formation rates, heat and fresh water transports, sea surface temperature anomalies, etc.) of individual basins or the world ocean. The strong dissipation required to maintain numerical stability in these low resolution models inhibits realistic hydrodynamic instabilities as well. This class of models has been moderately successful in simulating the mean ocean circulation and hydrographic structure of the world ocean (e.g., Bryan, 1979), and the variability of the upper ocean circulation where the variability is primarily wind forced (e.g., Sarmiento, 1986; Philander et al., 1987). The inverse model studied in this thesis will be first examined in one of these numerical OGCM oceans. 
On the other hand, there are models with high horizontal resolution, but low vertical resolution, and generally incomplete treatment of thermodynamic processes (the Eddy-resolving General Circulation Models-EGCMs). These have been developed in order to investigate the dynamics of time-dependent circulation systems including mesoscale eddies and their interactions with the mean flow (e.g., Holland, 1986). Thermohaline processes are difficult to incorporate into these models and they are limited to non-global domains in the early stage.

Advances in computer power are leading to a blurring of the distinction between the OCGMs and the EGCMs, and have facilitated the convergence of these two modeling approaches. Basin- to global-scale simulations which include both representation of thermodynamic processes responsible for water mass formation and sufficient horizontal resolution to allow the hydrodynamic instabilities responsible for eddy formation have become feasible (e.g., Semtner and Chervin, 1988; F. Bryan and Holland, 1989). The inverse model studied in this work will also be tested in one of these GCM oceans (we will label it with EGCM).

Schott and Stommel's original $\beta$-spiral method (without diffusion in the approximate density conservation equation) was tested by Bigg (1985) in a non-eddy resolving numerical GCM ocean. The inverse model estimated beta-spirals are far apart from the GCM "data", with typical differences of $0.5 \mathrm{~cm} / \mathrm{s}$, although the shape of the spirals are qualitatively similar (Fig. 1.1). With diffusion added to the density equation (which was included in the numerical GCM), the model velocities are improved toward the "data", but the offsets are still significantly large. Also, the differences of the diffusivities $A$ (horizontal) and $K$ (vertical) between the inversion and the "data" are quite significant, and they are sensitive to the number of the levels in the inverse model as well as the choice of the reference levels. 
An intercomparison of three inverse models (the box model of Wunsch, 1978; the beta-spiral model of Schott and Stommel, 1978; and the Bernoulli model of Killworth, 1986) was made by Killworth and Bigg (1988) in the domain of a traditional (with highly simplified topography) eddy-resolving general circulation model (EGCM) ocean (Cox, 1985). Two "scores" were defined for the inverse models: one tests point-wise accuracy (the "global" score), and the other tests flux of mass through a section (the "flux" score). They concluded that the Bernoulli method yields accurate global scores except in the homogenized region; the box inversion yields fairly accurate global scores everywhere, and the beta-spiral only gives accurate global scores near the equator. No method gives reliable flux scores, although the box inverse was the least inaccurate. The estimated velocities by the beta-spiral method are different from the GCM data (Fig. 1.2). In this figure, the arrows are the $\beta$-spiral method estimated velocity vectors, whereas the ellipses are the $5 \%$ errors of the corresponding time-mean GCM velocity vectors (from the tails of the shown vectors to the centers of the ellipses). More profoundly, they showed that a hypothesis of no flow at the ocean bottom gives predicted velocity fields (by thermal wind relation) which are closer to the "data" than any of the inversions in most cases.

Although the GCM statistical errors were shown, no estimated error information from the inversions was available, and as a result, we cannot judge the significance of the above velocity differences. Possible reasons for the "failure" of the inverse models in their GCM oceans will be analyzed in Chapter 5, in comparison with our conclusions. 

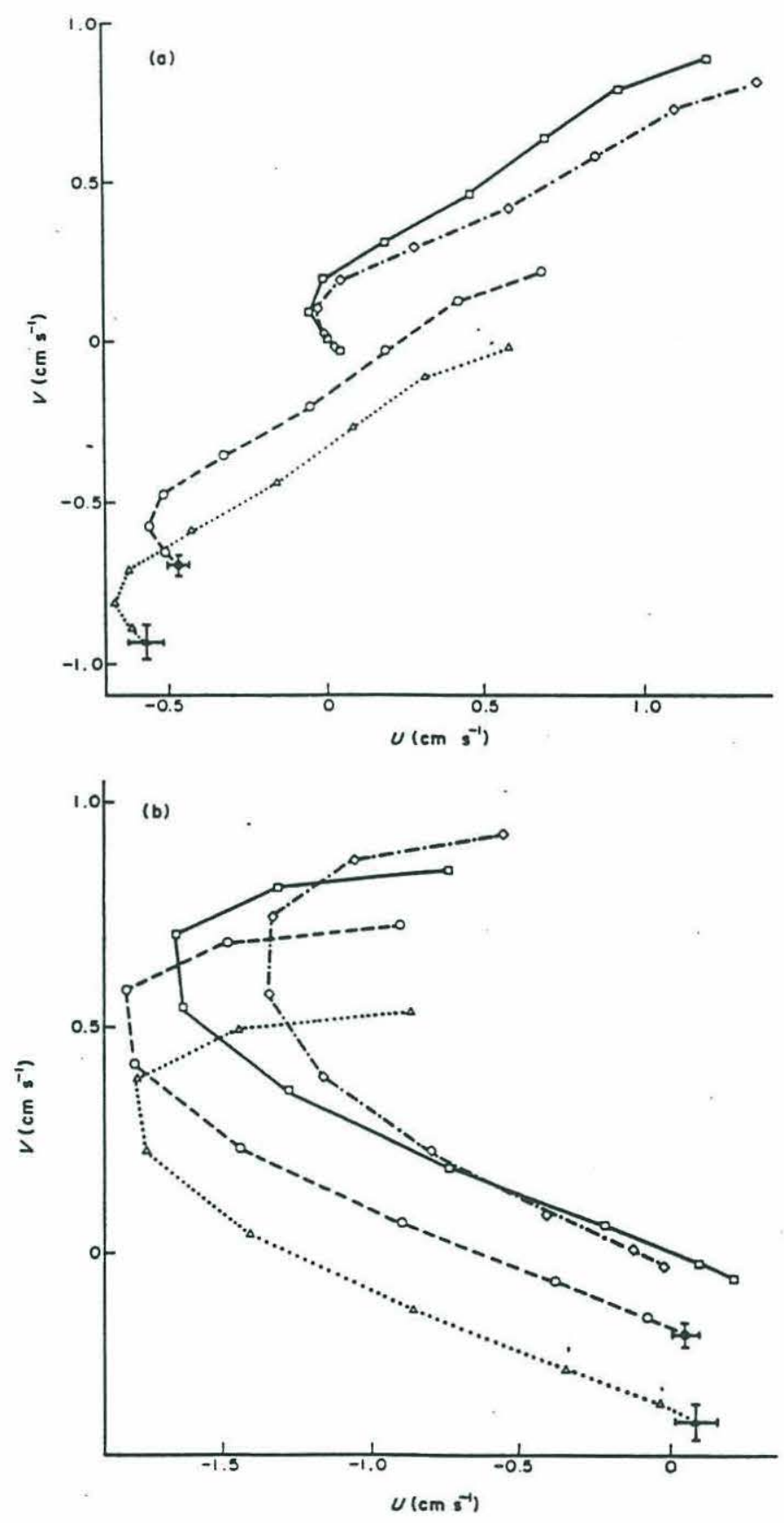

Fig. 1.1. Absolute velocity profiles at two points of a GCM ocean and various $\beta$-spiral inversions.

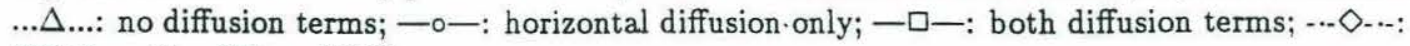
GCM profile. (Bigg, 1985) 

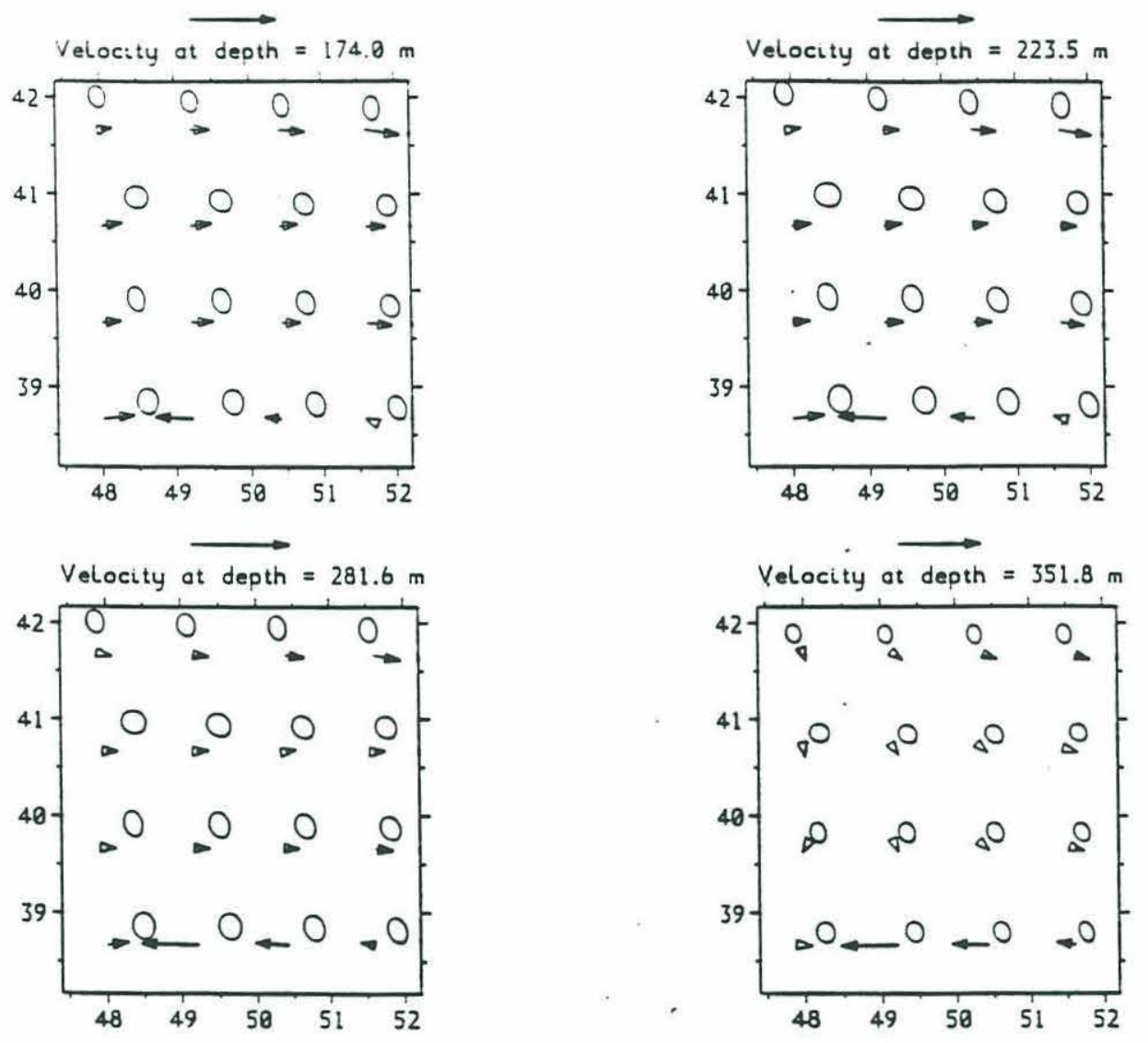

Fig. 1.2. Velocity vectors from a mass-conserving $\beta$-spiral inversion. The scale of $1 \mathrm{~cm} / \mathrm{s}$ is shown. The tail of the vector is at the point concerned; the ellipse shows the $5 \%$ error $(95 \%$ score) boundary. (Killworth and Bigg, 1988) 


\subsection{THE APPROACH OF THIS WORK}

The conclusions of the above two papers raises doubts about the reliability of the inverse models, especially those derived from the beta-spiral method. A complete analysis of inverse model results should include all the error information. Oceanography has reached a stage of maturity such that estimates of parameter values without corresponding estimates of error no longer seem very useful. It should also be pointed out that, first of all, inverse model results to some extent depend on specific inverse techniques (e.g., scalings of the equations-row scaling, and scalings of the unknowns-column scaling). Secondly, the original beta-spiral method does not include the conservation equations of heat and salt as well as other tracers. Adding these conservation constraints will provide more information and the parameter solutions should be improved in terms of statistical closeness (with estimated uncertainties) to their "true" values, and/or in terms of the socalled solution resolution, which indicates how well the parameters are resolved (for detailed discussions, see Wunsch, 1989, or Zhang and Hogg, 1992). The reliability of this kind of inverse model has not been examined, and this is the objective of this work. Also, with all the information available from the numerical GCM results, detailed study of the terms controlling the inverse solutions (the effects of the data "noise") and appropriate interpretation of the estimations will be examined. Experiments on the parameterization of some variables will also be carried out in the hope of getting some guidance in applying the inverse model to the real ocean.

The term "validation" of a model by a dataset must be used with caution. If the data physics is different from that of the model, we can not say that the model is "validated" by the data. Comparing the physics of the data and the model physics 
is the first step toward examining a model. In this study, the analysis involves three "oceans": the real ocean (I), the forward GCM ocean (II) aiming at predicting the evolution of the real ocean, and the inverse model ocean (III) which was originally introduced to reveal the major features of the real ocean from hydrographic data and other observations. The three "oceans" usually do not have exactly the same physics and statistics, and they should be distinguished among each other. The question of whether (II) properly (within the statistics) represents (I) should be separated from whether (III) properly represents (I) or (II).

In testing an inverse model in the domain of the GCM oceans, we should first examine how accurately the inverse model reproduces the GCM oceans, or whether (III) sufficiently represents (II) within the statistical confidence limits. If the answer is yes, we would expect that the statistical inference by (III) from the hydrographic data of (II) should produce/recover the correct answers of (II). Otherwise we must conclude that the inverse model "failed", which is unlikely. On the other hand, if there are some discrepancies between the physics of (III) and (II), not all the parameters could be produced "correctly". The most interesting part in this case is how "well" each parameter is reproduced. In other words, we should ask which parameters are "correctly" produced (i.e. statistically consistent with their "true" values), and which are not. Note that in this case even though the inverse model "failed" to produce the "correct" answers for the GCM ocean, one cannot conclude that the inverse model would also fail to produce the correct answers for the real ocean. As pointed out before, GCM oceans differ from the real ocean. Failure of properly representing (II) by (III) does not necessarily imply failure of properly representing (I) by (III). Consistent statistical inferences can still be achieved for the real ocean if it can be properly represented by the inverse model. 
The inverse model being tested in this work was originally developed by Hogg (1987) in isopycnal (potential density) coordinates. The major assumptions in this model are that the large scale oceanic circulations are in approximate geostrophic and hydrostatic balance, and mass, heat, potential density, and other tracers are approximately conserved in steady state. This model is of finite difference type.

In most traditional inverse models, velocities at the reference level are the unknowns, and the velocities at other depths are computed from the referencelevel velocities after the inversion through the exact satisfaction of thermal wind relation. Hogg's model solves for the absolute velocities on all the depths (levels) simultaneously. Like the conservation equation (of heat, potential density, oxygen) constraints, the dynamic equation (thermal wind relation) is just another constraint (on the absolute lateral circulations), and the equations are solved simultaneously. Residuals are allowed in the dynamic equation as well as in the conservation equations, and exact satisfaction of geostrophy/thermal wind relation is relaxed.

In Zhang and Hogg's (1992) (hereafter as ZH) application of this inverse model in the Brazil Basin, several modifications of the inverse model have been made. In Hogg's (1987) formulation and application in the central North Atlantic ocean, the Montgomery streamfunction, which was formulated for the specific volume or specific volume anomaly surfaces (Montgomery, 1938), was used on isopycnal surfaces in its original form. What is implied in this application is the neglect of the variation of specific volume (anomaly) along isopycnal (potential density) surfaces. In $\mathrm{ZH}$ it was shown that this variation may have dynamic importance in some regions of the ocean. By including the major part of this variation, new streamfunctions for the isopycnal surfaces were proposed. The second modification of the inverse model is in the ways of using the conservation equations. Hogg 
(1987) used a simplified conservation equation for potential density together with the use of conservation equation for heat. The implicit assumptions used were that the thermal/saline expansion/contraction coefficients are constant (independent of temperature and salinity and thus of space), and that both the horizontal and vertical diffusion coefficients for heat and salt (and other tracers like oxygen) are the same. These might not be true in some regions of the ocean, especially where double diffusion occurs (e.g., McDougall, 1987; Schmitt, 1994). In addition, potential density is a derived quantity from potential temperature and salinity through the equation of state. Therefore, to avoid possible errors from the above approximations, $\mathrm{ZH}$ used conservation equations for both heat and salt instead of for heat and the simplified density (among the three only two are independent). In the multilayer model (total of eight vertical layers extending from $250 \mathrm{~m}$ depth to $3500 \mathrm{~m}$ depth) in the Brazil Basin, $\mathrm{ZH}$ found that the inverse model estimated circulations in the upper ocean are consistent with previous studies (e.g., Reid, 1989; Defant, 1941). In the deep ocean, solutions are consistent with some previous work (e.g., Defant, 1941; Fu, 1981), but differ from others in small scale structure (e.g., Reid, 1989).

Experience in using this inverse model in the real oceans (and also in the numerical GCM oceans in the later chapters of this work) shows that this model normally results in an overdetermined system (in the traditional sense) and of full rank. Thus the solutions are obtained by minimizing the equation residual norm (in the least square sense), and no constraints on the parameter (solution) norm are used. In an underdetermined system, or an apparently overdetermined system with deficient rank, the solution (parameter) norm, or a combination of solution norm and equation residual norm is minimized in obtaining the solutions. For the 
problems formulated for reference velocity what is usually minimized is the kinetic energy at the reference level and thus the choice of the depth of the reference level is important in these cases. In this sense Hogg's formulation is more objective but there are still subjective factors as well in this model (like the row scaling).

The thesis is organized in the following way. First, the physics and assumptions used in both the numerical ocean general circulation model and the inverse model are briefly described and compared in Chapter 2 . Then the inverse model is applied to a simple, non-eddy resolving GCM ocean (Chapter 3), to see how well it functions there. Several issues, such as the Ekman pumping velocity constraint, determining the air-sea heat and fresh water fluxes, effects of data "noise" on the solutions, the effects of temporal variation terms, as well as parameterization of diffusive variables, are pursued in this chapter. In Chapter 4, the results of the application of the inverse model in a more recent CME (EGCM) ocean are discussed. The model is first applied to the time-mean fields of the numerical GCM ocean, with the fine grid resolution of the GCM. The inverse model estimated circulations are compared with the time-mean GCM circulations, to see the ability of the inverse model to recover the time-mean circulations from time-mean hydrographic data. Also compared are the "eddy" diffusion coefficients from the inverse and those from direct computations of the eddy fluxes. The next part of this chapter examines effects of spatial smoothing and larger grid spacing, which are usually used in the real ocean, on inverse model solutions. Finally, discussions and conclusions are given in Chapter 5. 


\section{Chapter 2. THE MODELS}

\subsection{INTRODUCTION}

As described in the previous chapter, the main purpose of this work is to examine whether useful inferences of the ocean state can be made by an inverse model from hydrographic data. Because of the lack of the needed information in the real ocean with which to compare the inverse model results, the examination is based on the numerical ocean general circulation model modeling results. In this study, the fields of water properties (potential temperature, salinity etc.) of simplified oceans generated by numerical ocean general circulation models (GCMs) are used as data for the inverse model, and the parameters for horizontal and vertical velocities and horizontal and vertical diffusion coefficients are estimated by solving the inverse model equations. Although these parameters are also known in the idealized numerical oceans, they are not used to constrain the inverse model solutions. Instead, they are used to compare with the inverse model results. This procedure is based on the notion that if the inverse model were applied to the real ocean, such information is generally not available.

As mentioned in Chapter 1, in order to fulfill the above objectives and to make meaningful analyses of the inverse model results, a complete understanding of the physics and assumptions of both the numerical GCM and the inverse model is essential. If the inverse model has the same physics and assumptions as the numerical GCM, or the differences are numerically negligible, we would expect the inverse model estimations to be completely consistent with those of the numerical GCM ones statistically. This total consistency of the inverse model physics with 
those producing the input data is difficult to achieve. For one reason, the GCM oceans are not completely in steady state, while the inverse model is a steady one. For another, the momentum equations in the GCM are unsteady and nonlinear, while the inverse model assumes geostrophic and thermal wind balances. With these subtle differences of the physics and assumptions, the question of whether we have the ability to utilize inverse techniques to make useful parameter estimations for horizontal and vertical velocities, diffusion coefficients, as well as air-sea heat and fresh water fluxes when the surface layer is included, is the main theme of this study.

First the numerical ocean general circulation model will be briefly described. Then the assumptions and formulations of the inverse model will be introduced. Finally, comparison of the two models will be made.

\subsection{THE NUMERICAL OCEAN GENERAL CIRCULA- TION MODEL}

The numerical ocean general circulation model used to simulate the ocean circulations and water property distributions is the three-dimensional primitive equation model of the ocean developed by the Geophysical Fluid Dynamics Laboratory (GFDL) of NOAA at Princeton University (Bryan, 1969; Cox, 1984). The model momentum equations are the simplified non-steady, non-linear, Navier-Stokes equation with three basic assumptions: the Boussinesq approximation, in which density differences are neglected except in the buoyancy term; the hydrostatic assumption, in which the equation of vertical motion is simplified by neglecting local acceleration and other terms of equal or smaller order; and the turbulent viscosity hypothesis 
which is used to parameterize the stresses exerted by sub-grid scale motions. Water property changes (potential temperature, salinity, etc.) are obtained by integrating the non-steady conservation equations forward in time, again utilizing a turbulent mixing hypothesis to represent the sub-grid cell processes. The equations are linked by a simplified, but nonlinear equation of state.

In spherical coordinates, the continuous form of the equations are as follows:

$$
\begin{aligned}
\frac{\partial u}{\partial t}+\frac{m}{R_{e}}\left[\frac{\partial(u u)}{\partial \lambda}+\frac{\partial(v u / m)}{\partial \phi}\right]+\frac{\partial(w u)}{\partial z}-f v & =-\frac{m}{R_{e}} \frac{\partial\left(p / \rho_{0}\right)}{\partial \lambda}+F^{u} \\
\frac{\partial v}{\partial t}+\frac{m}{R_{e}}\left[\frac{\partial(u v)}{\partial \lambda}+\frac{\partial(v v / m)}{\partial \phi}\right]+\frac{\partial(w v)}{\partial z}+f u & =-\frac{1}{R_{e}} \frac{\partial\left(p / \rho_{0}\right)}{\partial \phi}+F^{v} \\
p(z) & =p^{s}+\int_{z}^{0} g \rho d z \\
\frac{m}{R_{e}}\left[\frac{\partial u}{\partial \lambda}+\frac{\partial(v / m)}{\partial \phi}\right]+\frac{\partial w}{\partial z} & =0 \\
\frac{\partial T}{\partial t}+\frac{m}{R_{e}}\left[\frac{\partial(u T)}{\partial \lambda}+\frac{\partial(v T / m)}{\partial \phi}\right]+\frac{\partial(w T)}{\partial z} & =F^{T} \\
\rho & =\rho(\theta, S, z)
\end{aligned}
$$

where $\phi$ is the latitude, $\lambda$ the longitude, $m=\sec \phi, n=\sin \phi, R_{e}$ the radius of the earth, $f$ the Coriolis parameter, $p^{s}$ the pressure at the surface of the ocean, $T$ the concentration of any "tracer" type quantity (like active tracers potential temperature $\theta$ and salinity $S$, and passive tracers tritium and carbon 14, etc.), and $F^{u}, F^{v}$, and $F^{T}$ the dissipation by processes with scales too small to be resolved by the finite difference grid resolutions. These sub-grid scale processes are parameterized by a second order operator in the vertical and a Laplacian operator in the horizontal of the following forms: 


$$
\begin{aligned}
F^{u} & =A_{M H} R_{e}^{-2}\left[\nabla^{2} u+\left(1-m^{2} n^{2}\right) u-2 n m^{2} \frac{\partial v}{\partial \lambda}\right]+A_{M V} \frac{\partial^{2} u}{\partial z^{2}} \\
F^{v} & =A_{M H} R_{e}^{-2}\left[\nabla^{2} v+\left(1-m^{2} n^{2}\right) v+2 n m^{2} \frac{\partial v}{\partial \lambda}\right]+A_{M V} \frac{\partial^{2} v}{\partial z^{2}} \\
F^{T} & =A_{T H} R_{e}^{-2} \nabla^{2} T+\frac{\partial}{\partial z}\left(\frac{A_{T V}}{\delta} \frac{\partial T}{\partial z}\right)
\end{aligned}
$$

where

$$
\begin{aligned}
\nabla^{2} T & =m^{2} \frac{\partial^{2} T}{\partial \lambda^{2}}+m \frac{\partial\left(\frac{\partial T}{\partial \phi} / m\right)}{\partial \phi} \\
\delta & =1, \text { for } \frac{\partial \rho}{\partial z}<0 \\
\delta & =0, \text { for } \frac{\partial \rho}{\partial z}>0
\end{aligned}
$$

and $A_{a b}$ is the mixing coefficient for momentum and tracers (denoted by the first subscript $M$ and $T$ ) and in the horizontal and vertical directions (denoted by the second subscript $H$ and $V$ ). Note that vertical mixing is specified to be uniform under statically stable conditions $\left(\frac{\partial \rho}{\partial z}<0\right)$, and to be infinite under statically unstable conditions $\left(\frac{\partial \rho}{\partial z}>0\right)$. In the eddy-resolving numerical GCM whose results will be used in Chapter 4, the second order Laplacian dissipation described above is replaced by a fourth order biharmonic horizontal dissipation. The equation of state, eq.(2.6), is approximated as a nine-term, third-order polynomial in temperature and salinity (Bryan and Cox, 1972).

The "rigid-lid" assumption of zero vertical velocity at the surface of the ocean and the assumptions of no normal flow and no normal tracer fluxes at solid boundaries are adopted. Specifications of the values of turbulent viscosities $A_{M H}$ and $A_{M V}$, and diffusivities $A_{T H}$ and $A_{T V}$, as well as the surface boundary conditions (wind stress, air-sea heat and fresh water fluxes) will be discussed later together with the analyses of the corresponding numerical GCM results. 
The finite difference formulation and the arrangement of variables within the cells correspond to the "B-grid" configuration of Arakawa and Lamb (1977). Horizontally, the grids for $T$ and the grids for $u, v$ are staggered, with $T$ grids situated in the centers of the cells, and $u, v$ grids placed at the corners of the cells (Fig. 2.1a). Vertically, the grids for $T, u, v$ are located halfway through the vertical dimension of the cells, while the grids for $w$ are located at the horizontal interfaces of the cells (Fig. 2.1b). Two sets of vertical velocities, $w^{T}$ and $w^{u}$, are calculated through the diagnostic continuity equation, at the interfaces of cells, and in the vertical lines of $T$ grids and $u, v$ grids respectively. The quantity $w^{T}$ is used in the tracer conservation equation for computing $T$, while $w^{u}$ is used in the momentum equations for computing $u$ and $v$. In writing the equations in finite difference form, the central-difference scheme is generally used. Further information can be found in the GFDL documentation (Cox, 1984). 


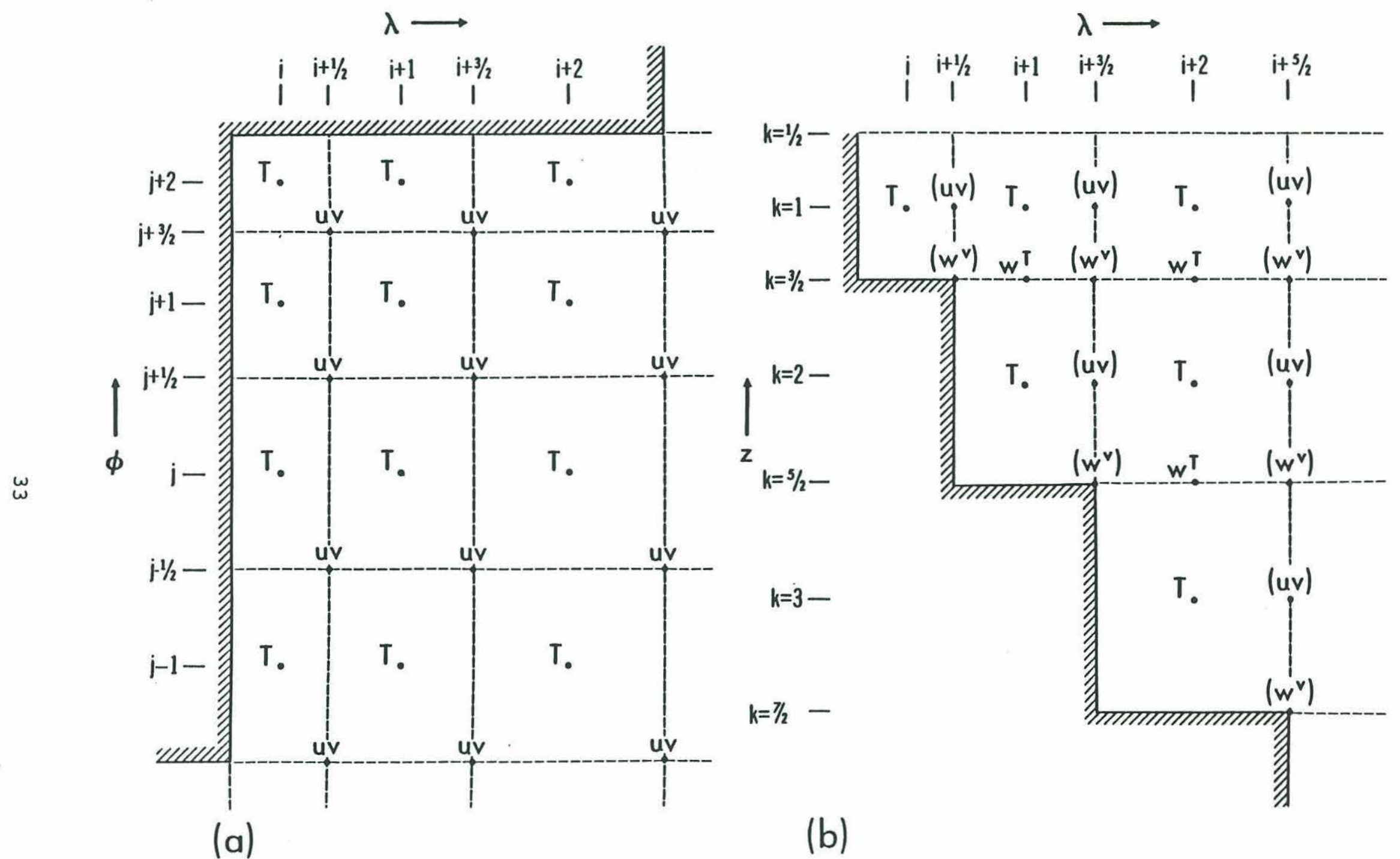

Fig. 2.1. Staggered grids for the centered finite difference scheme for the governing equations in the GCM and the inverse model. (Cox, 1984). 


\subsection{THE INVERSE MODEL}

\subsubsection{Introduction}

The inverse model to be examined is a combination of dynamic relationship and steady state water property conservation laws, written in point-wise finite difference form (Hogg, 1987) (properties are conserved in all the finite difference boxes). The basic assumptions of this model are: approximate geostrophic and hydrostatic balances, mass conservation (continuity equations) and additional conservation laws for tracers (potential temperature, salinity, etc.), and steady state. As in the numerical GCM, processes with scales too small to be resolved by the grid are parameterized as turbulent diffusive processes. The turbulent diffusion coefficients can be constant, as in the numerical GCM used in this work, or functions of space $(x, y, z)$.

This inverse model was originally designed for an isopycnal coordinate system. In this study, the numerical ocean data are generated by numerical GCMs in the geopotential $z$-coordinate. Therefore, to apply the inverse model described above, we either interpolate the numerical GCM data onto the potential density surfaces, if the finite difference resolutions of the GCMs permit, or reformulate the inverse model for the geopotential $z$-coordinate. Limited vertical resolution in the numerical GCM results (especially the non-eddy resolving one used in the next chapter) prevents the construction of reliable potential density surfaces and reliable interpolation of the numerical data. The better choice for this study is to reformulate the inverse model for the $z$-coordinate. In Martel and Wunsch's (1993) finite difference inverse model, the equations were also written in the $z$-coordinate. 


\subsubsection{Formulation of the Equations}

Dynamic Equation. Formulation of the Dynamic Equation starts with the hypothesis of geostrophic balance for horizontal velocities and the hydrostatic approximation (for the vertical momentum equation):

$$
\begin{aligned}
-f v & =\frac{-1}{\rho_{0}} \frac{\partial p}{\partial x} \\
f u & =\frac{-1}{\rho_{0}} \frac{\partial p}{\partial y} \\
\frac{\partial p}{\partial z} & =-g \rho .
\end{aligned}
$$

The vertical difference (shear) of the horizontal velocity at two depths can be then derived as

$$
\begin{aligned}
f\left(v_{1}-v_{2}\right) & =\frac{1}{\rho_{0}} \frac{\partial\left(p_{1}-p_{2}\right)}{\partial x}=\frac{1}{\rho_{0}} \frac{\partial}{\partial x} \int_{p_{2}}^{p_{1}} d p \\
& =\frac{1}{\rho_{0}} \frac{\partial}{\partial x} \int_{z_{2}}^{z_{1}}-g \rho d z=\frac{-g}{\rho_{0}} \frac{\partial}{\partial x} \int_{z_{2}}^{z_{1}} \sigma d z \\
& =\frac{\partial}{\partial x}\left[\frac{-g}{\rho_{0}} \int_{z_{2}}^{z_{1}} \sigma d z\right], \\
f\left(u_{1}-u_{2}\right) & =-\frac{\partial}{\partial y}\left[\frac{-g}{\rho_{0}} \int_{z_{2}}^{z_{1}} \sigma d z\right]
\end{aligned}
$$

where $\sigma=\rho-1000$ is the density anomaly. Define a streamfunction $\psi$ for the quantity $f \vec{u}$ such that

$$
\begin{aligned}
f u & =-\frac{\partial \psi}{\partial y} \\
f v & =\frac{\partial \psi}{\partial x} .
\end{aligned}
$$

From the relations above it is obvious that a natural choice for the streamfunction for $f \vec{u}$ in $z$-coordinate is

$$
\psi=d h \equiv \frac{-g}{\rho_{0}} \int^{z} \sigma d z
$$


In this work, we will call the quantity $d h$ defined above the "dynamic height" for the geopotential $(z)$ surfaces. Physically it is a anomaly pressure surface. Note that this $d h$ is different from the conventional dynamic height $-\int^{p} \delta d p$, which is the streamfunction for the isobaric surfaces.

Continuity and Vorticity Equations. In the mass conservation equation, the compressibility of the sea water is generally much smaller than the velocity divergence term, and therefore can be ignored. Mass conservation is represented by the three-dimensional continuity equation:

$$
\nabla \cdot \vec{u}+\frac{\partial w}{\partial z}=0
$$

where the $\nabla$. is the two-dimensional divergence operator in the horizontal plane.

One additional constraint which could be included in the inverse model equations is the so called integrated vorticity constraint. The equation for this constraint can be derived by integrating the linear vorticity equation. The vertical arrangement of the grid points are such that, as in the numerical GCM (Fig. 2.1), grid points for $T, u, v$ are at halfway through the cell, and grid points for $w$ are at the interfaces of the cells. Let $z w_{k}, z w_{k+1}$ denote the depths of the $k^{\text {th }}$ and $k+1^{\text {th }}$ levels of $w$, and $z T_{k}$ the depth of the $k^{\text {th }}$ level of $T, u, v$. Then $z T_{k}$ is in the middle of $z w_{k}$ and $z w_{k+1}$. The thermal wind relation and hydrostatic approximation result in

$$
v(z)-v\left(z T_{k}\right)=\frac{1}{\rho_{0} f} \frac{\partial}{\partial x}\left[p(z)-p\left(z T_{k}\right)\right]=\frac{-g}{\rho_{0} f} \frac{\partial}{\partial x} \int_{z T_{k}}^{z} \sigma d z .
$$

Using the above relation and integrating the following linear vorticity equation

$$
\beta v=f \frac{\partial w}{\partial z}
$$


from $z w_{k}$ to $z w_{k+1}$ yields the Integrated Vorticity equation:

$$
\begin{aligned}
w\left(z w_{k+1}\right) & -w\left(z w_{k}\right)=\frac{\beta}{f} \int_{z w_{k}}^{z w_{k+1}} v(z) d z \\
& =\frac{\beta}{f} \int_{z w_{k}}^{z w_{k+1}}\left[v\left(z T_{k}\right)+\frac{-g}{\rho_{0} f} \frac{\partial}{\partial x} \int_{z T_{k}}^{z} \sigma d z\right] d z \\
& =\frac{\beta}{f}\left[v\left(z T_{k}\right)\left(z w_{k+1}-z w_{k}\right)+\frac{-g}{\rho_{0} f} \frac{\partial}{\partial x} \int_{z w_{k}}^{z w_{k+1}}\left(\int_{z T_{k}}^{z} \sigma d z\right) d z\right] .
\end{aligned}
$$

Conservation Equation for Tracers. Parameterizing the sub-grid scale processes by Fickian diffusion with isotropic horizontal diffusion coefficients, the steady state conservation equations for water properties can be written as

$$
\nabla \cdot(\vec{u} T)+\frac{\partial(w T)}{\partial z}=\nabla(A \nabla T)+\frac{\partial}{\partial z}\left(K \frac{\partial T}{\partial z}\right)
$$

The horizontal and vertical turbulent diffusion coefficients $A$ and $K$ can be parameterized as constants or functions of space.

In the tracer conservation equations, advection terms are usually much larger than diffusion terms in the ocean. In order to get better estimations for the diffusion coefficients from the inverse model, the tracer concentration $T$ is replaced by its local anomaly $T^{\prime}$, where $T^{\prime}=T-\bar{T}$, and $\bar{T}$ is a constant, taken as the horizontal mean at the depth concerned (see Hogg, 1987, Zhang and Hogg, 1992 for further discussion).

The estimation of the velocity, especially the zonal component, will also benefit from this substitution. In order to explain this point, we decompose the the horizontal tracer flux divergence into two terms for a scale analysis (in the real computation, no decomposition is used):

$$
\begin{aligned}
\nabla(\vec{u} T) & =\vec{u} \cdot \nabla T+T \nabla \cdot \vec{u} \\
& \approx \vec{u} \cdot \nabla T-T \frac{\beta}{f} v
\end{aligned}
$$


where the $\beta$-plane geostrophic approximation has been used in the second step. Note that the coefficient for $u$ only involves the $x$-gradient, $\partial T / \partial x$, while the coefficient for $v$ involves both the gradient, $\partial T / \partial y$, and the tracer concentration $T$ itself. Their ratio can be estimated via scale analysis:

$$
R=\frac{T \beta v / f}{\vec{u} \cdot \nabla T} \sim \frac{T U \cot \phi / R_{e}}{U \Delta_{x y} T /\left(L_{x}, L_{y}\right)}=(\Delta x, \Delta y) \frac{\pi}{180} \cot \phi \cdot \frac{T}{\Delta_{x y} T} \sim .06 \times \frac{T}{\Delta_{x y} T}
$$

where $L_{x}, L_{y}$ are the grid distances and $\Delta x, \Delta y$ are the corresponding grid sizes in degrees. $\Delta_{x y} T$ is the scale of the concentration differences at one-grid size. In the last step the number is estimated by using $2^{\circ}$ grid size and at mid-latitude, $\phi=30^{\circ}$.

For salinity, a typical value of $S$ is $35 p s u$, and a typical value of $\Delta_{x y} S$ is of $0.1 \mathrm{psu}$. These numbers give a typical value of 20 for $R$. For temperature, choices of $T=20^{\circ}$ and $\Delta_{x y} T=0.1$ result in a value of 12 for $R$. In both cases, the coefficient for $v$ is much larger than the coefficient for $u$, which makes accurate estimation for $u$ much more difficult. On the other hand, if $T$ is replaced by $T^{\prime}$, its easy to see the ratio is greatly reduced and typically $R<O(1)$, and the coefficients for $u$ and $v$ are of the same magnitude.

\subsubsection{Surface Layer Model}

The inverse model described above cannot be applied to the surface mixed layer, which is under the direct influence of wind stress and air-sea interactions. Geostrophic balance and the thermal wind relation obviously do not hold in this layer and special treatment is needed.

In one approach, if one is not interested in knowing the circulations in the surface layer and the air-sea heat and fresh water fluxes, one could use the Ekman 
pumping velocity as a constraint on the vertical velocity at the bottom of the Ekman layer. In practice, however, it is difficult to determine the Ekman layer depth at which the Ekman pumping velocity should be specified for the inverse model.

Another approach is to incorporate the Ekman theory into equations of mass and tracer conservations to estimate the circulations in the surface layer and the water property (heat, fresh water, etc.) fluxes through the air-sea interfaces. Vertically integrated horizontal mass transport (the Ekman transport) due to wind stress $\vec{\tau}$ can be derived as (e.g., Pedlosky, 1987)

$$
\vec{M}_{E}=\frac{\vec{\tau} \times \hat{k}}{\rho f} .
$$

If we assume that the horizontal velocity in the surface layer mainly consists of geostrophic velocity and wind driven velocity (this assumption will be examined in the next chapter), the mass conservation in the surface layer can be written as

$$
\Delta z_{1}\left(\frac{\partial u_{g}}{\partial x}+\frac{\partial v_{g}}{\partial y}\right)+\nabla \cdot \vec{M}_{E}+\left(w_{1}-w_{2}\right)=0,
$$

and the tracer conservation equation can be derived as

$$
\begin{aligned}
\nabla\left(\vec{u}_{g} T\right) & +\frac{\nabla\left(\vec{M}_{E} T\right)}{\Delta z_{1}}+\frac{\partial(w T)}{\partial z} \\
& -\nabla(A \nabla T)-\frac{F_{\text {surf }}-K\left(T_{1}-T_{2}\right) /\left(z T_{1}-z T_{2}\right)}{\Delta z_{1}}=0
\end{aligned}
$$

where $F_{\text {surf }}$ is the tracer (heat and fresh water) fluxes at the surface of the ocean. The quantities $w_{1}, w_{2}$ are the vertical velocities at the sea surface and at the bottom of the surface layer (taken as $50 \mathrm{~m}$ in the case in Chapter 3 ) respectively, while $T_{1}, T_{2}$ are the potential temperature at the middle depths of the surface layer $(25 \mathrm{~m}$ in the case in Chapter 3 ) and the second layer (75 meters). In the above equation, the unknowns are the parameters for geostrophic component of the horizontal circulation, vertical velocity (specified as zero at sea surface) and diffusion coefficients. If 
the surface fluxes are known accurately (e.g., from climatological data), they can be used as further constraint on the estimation of velocities and diffusion coefficients. Otherwise, they can be estimated from the above equation as well. Technically, this can be done by adjusting the weighting factors for these constraints.

\subsubsection{Finite Difference Formulation}

In summary, the constraints used in the inverse model are the dynamic equation. continuity equation, integrated vorticity equation and conservation equations for water properties (heat and salt):

$$
\begin{aligned}
& \psi_{k}-\psi_{k+1} \approx d h_{k \rightarrow k+1}=\frac{g}{\rho_{0}} \int_{k}^{k+1} \sigma d z \\
& \frac{\partial u}{\partial x}+\frac{\partial v}{\partial y}+\frac{\partial w}{\partial z} \approx 0 \\
& w\left(z w_{k+1}\right)-w\left(z w_{k}\right)-\frac{\beta}{f} v\left(z T_{k}\right)\left(z w_{k+1}-z w_{k}\right) \approx-\frac{\beta}{f^{2}} \frac{\partial}{\partial x} \frac{g}{\rho_{0}} \int_{z w_{k}}^{z w_{k+1}}\left(\int_{z T_{k}}^{z} \sigma d z\right) d z \\
& \left.\frac{\partial(u T)}{\partial x}+\frac{\partial(v T)}{\partial y}+\frac{\partial(w T)}{\partial z}-\nabla A \nabla T\right)-\frac{\partial}{\partial z}\left(K \frac{\partial T}{\partial z}\right) \approx 0
\end{aligned}
$$

For consistency, these equations are differenced using the same finite difference formulation as in the numerical GCM. For example, the advective flux terms are written as

$$
\begin{aligned}
& \frac{\partial(u T)}{\partial x}+\frac{\partial(v T)}{\partial y}+\frac{\partial(w T)}{\partial z}= \\
& \frac{\left(u_{i, j}+u_{i, j-1}\right)\left(T_{i+1, j}+T_{i, j}\right)-\left(u_{i-1, j}+u_{i-1, j-1}\right)\left(T_{i, j}+T_{i-1, j}\right)}{4 L_{x} \cos \left(\phi_{j}\right)} \\
& +\frac{\left(v_{i, j}+v_{i-1, j}\right)\left(T_{i, j+1}+T_{i, j}\right) \cos \left(\phi_{j+\frac{1}{2}}\right)-\left(v_{i, j-1}+v_{i-1, j-1}\right)\left(T_{i, j}+T_{i, j-1}\right) \cos \left(\phi_{j-\frac{1}{2}}\right)}{4 L_{y} \cos \left(\phi_{j}\right)} \\
& +\frac{w_{k+1}\left(T_{k}+T_{k+1}\right)-w_{k}\left(T_{k}+T_{k-1}\right)}{2\left(z w_{k+1}-z w_{k}\right)} .
\end{aligned}
$$


Note that in computing $T$ on the $w$ surfaces, a simple arithmetic average (instead linear interpolation of the non-constant vertical spacing) is used as in the GCM. In dealing with the real ocean, a linear (or distance-weighted) interpolation may be more realistic.

The unknowns to be estimated are the parameters for circulations (streamfunction), vertical velocity and turbulent diffusion coefficients. Streamfunction $(\psi)$ is an unknown which varies point-wise, and is placed at the $u, v$ grid points as in Fig. 2.1. Vertical velocity $w$ is also kept as point-wise unknown and is placed at the same grid point as in Fig. 2.1 in most of the experiments. Diffusion coefficients can be parameterized as constants, or functions of space.

\subsection{SUMMARY}

For a better understanding of the inverse model results in the domain of the numerical GCM results, we should make clear the major differences in physics of the inverse model and the numerical GCM.

One major difference is that the numerical GCM is a prognostic model while the inverse model is a steady one. In the numerical GCMs, the parameters $u, v$ are predicted through the time-dependent nonlinear momentum equation (the primitive equation), and the tracer concentrations (potential temperature, salinity, etc.) are predicted through the time-dependent tracer conservation equations, with the aid of the diagnostic equations of continuity, hydrostatic assumption, equation of state as well as the specified turbulent viscosity and diffusivities. The vertical velocity is a diagnostic quantity and is computed from the continuity equation. 
In the inverse model, the momentum equation is a much simplified one: the circulation is (approximately) in geostrophic and thermal wind balances (in which the hydrostatic assumption and equation of state have been used). Steady state is assumed for all the parameters, thus reducing this to a diagnostic model. Distributions of sea-water properties are used to estimate the ocean circulations as well as the turbulent diffusion coefficients for the properties.

If the data generated by the numerical GCM satisfy the inverse model equations accurately, we would expect that the inverse model estimates of the parameters should be consistent with the values of the numerical GCM parameters. Any discrepancy in the inverse model results and the corresponding numerical GCM parameters should be accounted by the differences in the physics of the two models. More complete analyses will be seen in the next two chapters. 


\section{Chapter 3. APPLICATION OF THE INVERSE MODEL IN A NON-EDDY RESOLVING NUMERICAL GCM OCEAN}

\subsection{INTRODUCTION}

We will first examine the functioning of the inverse model in a simpler ocean-the ocean generated by a non-eddy resolving numerical GCM in this chapter. With a basic understanding of the inverse model results and its usefulness, the inverse model will be examined in a more realistic numerical ocean-the ocean produced by an eddy-resolving GCM, which is more mimic to the real ocean This will be done in the next chapter.

The non-eddy resolving numerical ocean was generated by running the numerical GCM described in the previous chapter with the following configuration (Spall, 1992). The finite difference grid resolutions are $2^{\circ}$ in latitude and longitude and a maximum of 10 levels in the vertical for tracers and horizontal velocities. The numerical model domain was the North Atlantic Basin, extending from the equator to $64^{\circ} \mathrm{N}$ and from $80^{\circ} \mathrm{W}$ to $10^{\circ} \mathrm{W}$ with "real" topography (Fig. 3.1). The horizontal resolutions were chosen to represent major current features in the North Atlantic Basin (such as the Gulf Stream, North Atlantic Current, deep western boundary current, and the gyre recirculations) while remaining computationally affordable for long time integrations. The depths at which tracers and horizontal velocities are located (the vertical centers of the cells) are at 25, 75, 150,300,500,800, 1250, 1750,2500 and 3500 meters. The interface depths of the cells, at which the vertical velocities are located, are at $0,50,100,200,400,600,1000,1500,2000,3000$ and 4000 meters. 
The turbulent coefficients of horizontal dissipation are $4 \times 10^{8} \mathrm{~cm}^{2} / \mathrm{s}$ (viscosity) and $1 \times 10^{7} \mathrm{~cm}^{2} / \mathrm{s}$ (diffusion), and the coefficients of vertical viscosity and diffusion are $1 \mathrm{~cm}^{2} / \mathrm{s}$. The heat flux at the surface of the ocean was parameterized by a relaxation of the temperature at the uppermost level in the model to the apparent atmospheric temperature. This apparent temperature is similar to the surface temperature of the ocean but takes into account radiative and evaporative effects (Han, 1984). The surface fresh water flux (evaporation minus precipitation) was parameterized by a relaxation of the salinity at the uppermost level of the model to the climatological mean surface salinity of Levitus (1982). The relaxation time constants for both temperature and salinity were chosen to be 100 days. The surface wind forcing was taken from the annual mean winds derived by Hellerman and Rosenstein (1983). The description of the boundary condition and initialization of the model as well as other information can be found in Spall (1992). The numerical ocean used to test the inverse model in this chapter is chosen as the final "equilibrium" state after 400 years of integration.

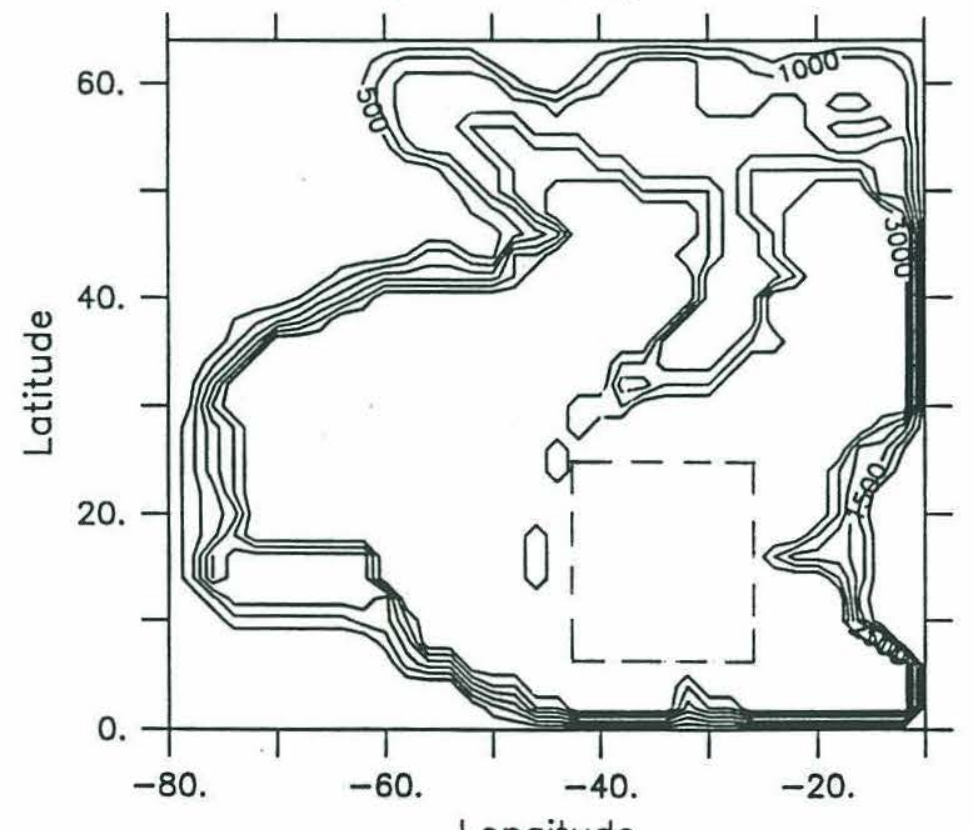

Fig. 3.1. Topography of the non-eddy resolving numerical GCM ocean. The dashed line indicates the inverse model domain. (Spall, 1992) 


\subsection{ACCURACY OF THE INVERSE MODEL ASSUMP- TIONS IN THE NON-EDDY RESOLVING GCM OCEAN}

The inverse model will be tested in a subdomain of the numerical GCM ocean, which extends from $42^{\circ} \mathrm{W}$ to $26^{\circ} \mathrm{W}$ and $7^{\circ} \mathrm{N}$ to $25^{\circ} \mathrm{N}$ (Fig. 3.1). This subdomain is so chosen that the assumptions of the inverse model tend to hold in this region and the topographic effects are minor, and in fact this region has a flat bottom of $4000 \mathrm{~m}$.

As was pointed out before, the numerical GCM data were generated by a full nonlinear prognostic numerical GCM, while the inverse model is a geostrophic and steady (diagnostic) model. We do not expect the numerical GCM data to satisfy the inverse model physics perfectly. We need to first determine how accurate the assumptions of the inverse model are in the numerical GCM ocean in order to fully understand the inverse model results.

\subsubsection{Dynamic Equation}

The dynamic equation in the inverse model was formulated from geostrophic and hydrostatic assumptions with their combination resulting in the thermal wind relation. From the available numerical data, the (vertical) shear of the horizontal velocity can be calculated in two ways: one is the real velocity shear computed directly from the GCM absolute velocities, and the other is the thermal wind shear computed from the the density field of the GCM ocean.

Comparisons of the two sets of calculations in the subdomain of the inverse model are shown in Fig. 3.2. In this figure, the first column (labeled with $u_{r}$ ) is the 
absolute velocity shear between two layer depths, and the second column $\left(u_{r} d y n\right)$ is the thermal wind shear. The third column is the difference (the residual) of the above two columns. Note that the contour intervals for the residuals (in the third column) are half of the contour intervals in columns one and two except in the top layer, in which the contour intervals are the same. The meridional velocity shears have similar features. It can be seen from these figures that the numerical GCM ocean circulations approximately satisfy the thermal wind relation except in the surface layer (at $25 \mathrm{~m}$ ) which is directly under the influence of wind stress. To be more quantitative, area-averaged ratios of the residuals over the shears are computed as

$$
\begin{aligned}
& R_{u}^{2}=\frac{\sum\left(u_{r}-u_{r} d y n\right)^{2}}{\sum u_{r}^{2}} \\
& R_{v}^{2}=\frac{\sum\left(v_{r}-v_{r} d y n\right)^{2}}{\sum v_{r}^{2}} .
\end{aligned}
$$

where the summation is taken over the horizontal inverse model domain.

The values of $R_{u}$ and $R_{v}$ in the inverse model domain and between the 10 model depths are shown in Table 3.1. Although visually (Fig. 3.2) the numerical GCM circulations are in good thermal wind relation, this table shows that the averaged deviation from the thermal wind relation can be as large as $20 \%$ in the zonal velocities. The meridional velocity has a better thermal wind relation, with averaged deviation of around $10 \%$. 
Table 3.1 Relative imbalances between the thermal wind shear and absolute velocity shear in the GCM ocean

$$
\begin{aligned}
& \text { Depth intervals (m) } \\
& 25-75-150-300-500-800-1250-1750-2500-3500 \\
& \begin{array}{llllllllll}
R_{u} & 1.40 & 0.14 & 0.29 & 0.24 & 0.13 & 0.07 & 0.23 & 0.09 & 0.09
\end{array}
\end{aligned}
$$

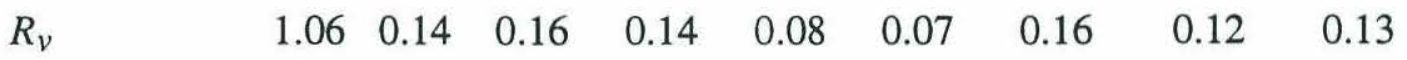

In addition to the intrinsic physical difference of the two kinds of velocity shear in Fig. 3.2, another source for the residuals in the figure and in Table 3.1 are the numerical errors in calculating the "dynamic height". By the definition in chapter 2, the "dynamic height" is an integral of the density anomaly in the vertical direction between two depths. As the data are usually available on the grid points only, the integrations in depth are usually carried out by fitting the data by polynomial functions. Limited vertical resolution of the numerical data will limit the accuracy of the interpolation and thus the integration. We used different orders of polynomial fit to the data in the vertical to calculate the dynamic height, which resulted in different velocity shear residuals defined above, although the variations in the dynamic height and the velocity shear themselves are small. The dynamic height used in Fig. 3.2 and Table 3.1 as well as in the inverse model estimations is calculated from the second order (linear) piece-wise $B$-splines polynomial fit to the data (de Boor, 1978), which yields the closest velocity shears to those in the numerical GCM data (better than higher order interpolations). Direct summation over the discrete GCM vertical levels (without interpolation) gives similar results to those with the 2nd order B-Spline fit. 
From the available numerical GCM data. we can also construct the terms in the momentum equation. Some of these terms are shown in Fig. 3.3 for the zonal velocity (The pressure gradient term is not computed because this computation requires special techniques to construct the sea surface pressure. But we would expect that this term will be similar to the Coriolis term but of opposite sign-no other terms in Fig.3.3 can balance the Coriolis term). From this figure it can be seen that the numerical ocean circulations are approximately in geostrophic balance except in the surface layer, and the ageostrophic part of the circulation mostly comes from the horizontal dissipation term. The area-averaged norm ratios $R_{d h u}$ and $R_{d h v}$ of the horizontal dissipation term over the Coriolis term defined by

$$
\begin{aligned}
R_{d h u}^{2} & =\frac{\sum A_{M H} \nabla^{2} u}{\sum(f v)^{2}} \\
R_{d h v}^{2} & =\frac{\sum A_{M H} \nabla^{2} v}{\sum(f u)^{2}}
\end{aligned}
$$

are shown in Table 3.2. It can be seen that these ratios are generally less than $6 \%$. The nonlinear terms are even smaller and are generally negligible, even in the surface layer.

Table 3.2 Area-averaged ratios of horizontal dissipation over the Coriolis force in the momentum equation.

\begin{tabular}{lllllllllllll} 
Depth (m) & \multicolumn{1}{c}{25} & -75 & -150 & -300 & 500 & -800 & -1250 & -1750 & $-2500-3500$ \\
$R_{d h u}$ & 0.02 & 0.03 & 0.02 & 0.03 & 0.04 & 0.03 & 0.06 & 0.05 & 0.03 & 0.05 \\
$R_{d h v}$ & 0.01 & 0.01 & 0.01 & 0.00 & 0.00 & 0.00 & 0.01 & 0.01 & 0.01 & 0.01 \\
\hline
\end{tabular}

In the surface layer, Ekman transport can be calculated from the wind stress. If we assume the GCM surface layer (from 0 to $50 \mathrm{~m}$ ) represents the Ekman layer, an 
averaged horizontal Ekman transport velocity at $25 \mathrm{~m}$ can be obtained by dividing the Ekman transport by the layer depth $(50 \mathrm{~m})$. At the same time, the geostrophic component in the absolute velocity at $25 \mathrm{~m}$ can be computed using the velocity at $75 \mathrm{~m}$ and the thermal wind relation. These two components compose the total horizontal velocity of the surface layer inverse model proposed in the previous chapter. Comparisons of the sums of the two components with the absolute GCM velocities at $25 \mathrm{~m}$ are shown in Fig. 3.4. The third column in this figure is their difference

(residual), and it can be seen that the residuals are much smaller than the values of the velocities themselves. The area-averaged norm ratios of the residuals over the velocity of the numerical GCM are $5.0 \%$ and $6.6 \%$ for $u$ and $v$ respectively. These numbers show that our model assumptions for the "momentum" equation in the surface layer are reasonably good and they can be used to estimate the surface heat and fresh water fluxes.

\subsubsection{Conservation Equation for Water Properties}

The numerical GCM is a prognostic model for the tracer conservation equations while the inverse model is a steady one. Although the numerical GCM used in this case is non-eddy resolving with steady forcing (steady wind stress and steady surface heat and fresh water fluxes), and the data were obtained after 400 years of integration, the fields were not in complete steady state. Using the numerical GCM data, the terms in the time-dependent temperature conservation equation at one instant (400 years) are shown in Fig. 3.5 at several depths. Terms in the salinity balance have similar patterns. 
In this figure the terms in the conservation equation are calculated for the tracer concentration anomaly $T^{\prime}$ instead of the concentration $T$ itself (see section 2.3.2 for detail). Replacing $T$ by $T^{\prime}$ only affects the sizes of the individual advection terms (namely $\nabla \cdot(\vec{u} T)$ and $(w T)_{z}$, they are smaller for $T^{\prime}$ than for $T$ ), but not their sum $\left(\nabla \cdot(\vec{u} T)+(w T)_{z}\right)$ and the diffusion terms, as the continuity equation is exactly satisfied by the GCM ocean.

It can be seen from this figure that the temporal variation terms are quite large compared to the diffusion terms, especially in the deep layers. When the steady inverse model is applied, these data "noises" will potentially bias the inverse model solutions, especially those for the diffusive parameters.

It should also be pointed out that, in the inverse model domain, the horizontal distributions of the temporal variation terms are much more scattered (although not totally randomly distributed) in space than those of the advection and diffusion terms. This somehow simulates the "randomness" of the data noise in the equations of the steady inverse model, and thus it is still possible to get meaningful spatiallysmoothed estimations for the parameters from the inverse model. It is also noticed that these temporal variation terms have similar horizontal structures on all the 10 vertical levels, and they are similar to the horizontal structures of the vertical advection terms of the deep levels. This is caused by the fact that, on levels 7,8 , and 9 (at the depths of $1250 \mathrm{~m}, 1750 \mathrm{~m}$ and $2500 \mathrm{~m}$ ), the major balance terms (for $T^{\prime}$ ) are the vertical advection and temporal variation variation. Therefore we would expected that neglecting the temporal terms in the inverse model will affect the estimation of the vertical velocity most significantly in the deep water. 


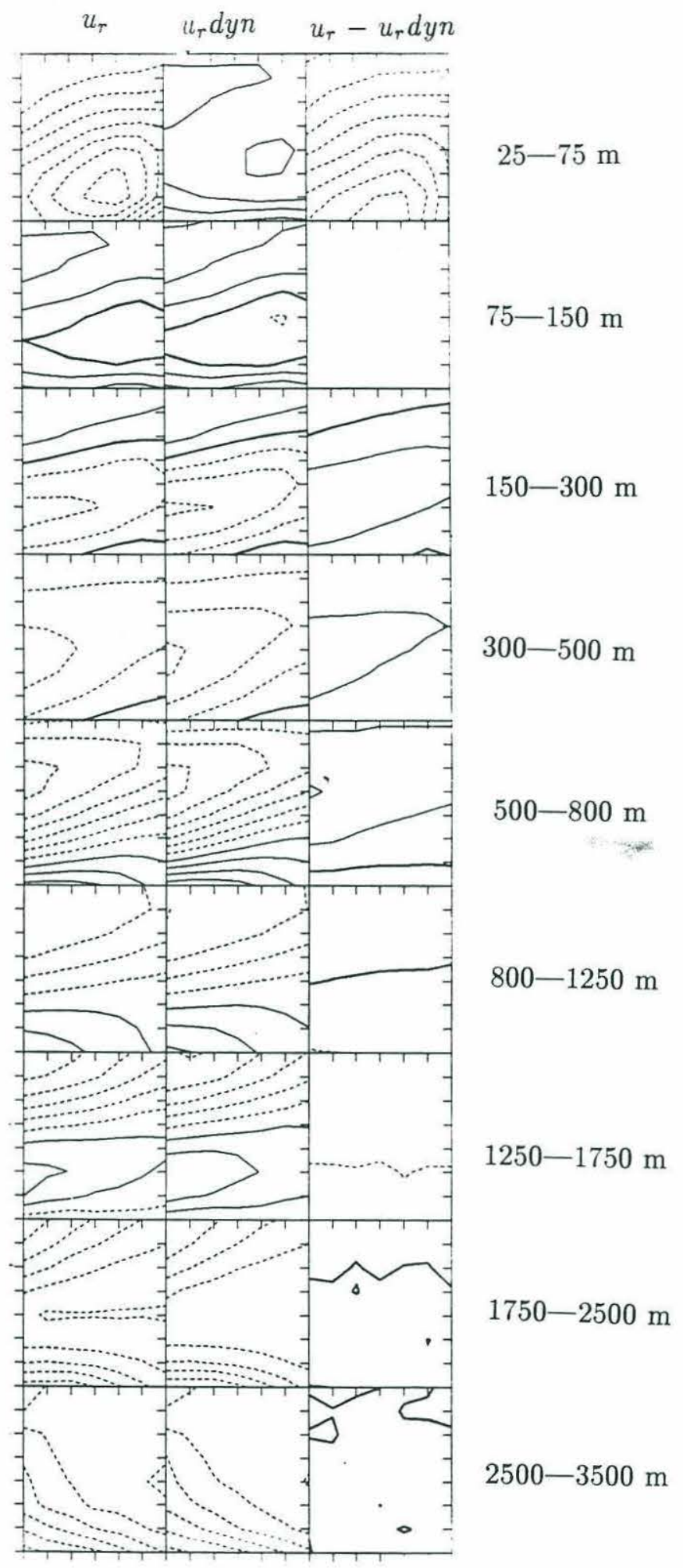

Fig. 3.2. The GCM absolute velocity shears $\left(u_{r}\right.$, the 1st column), thermal wind shears $\left(u_{r} d y n\right.$, the 2 nd column $)$, and their imbalances $\left(u_{r}-u_{r} d y n\right.$, the 3 rd column), between the numerical GCM layers. The contour intervals are $.5, .5, .5, .5, .2, .2, .05, .05 .05 \mathrm{~cm} / \mathrm{s}$ for the 9 layers for $u_{r}$ and $u_{r} d y n$, and half of those for their imbalances except at the top layer which is also .5 $\mathrm{cm} / \mathrm{s}$. 

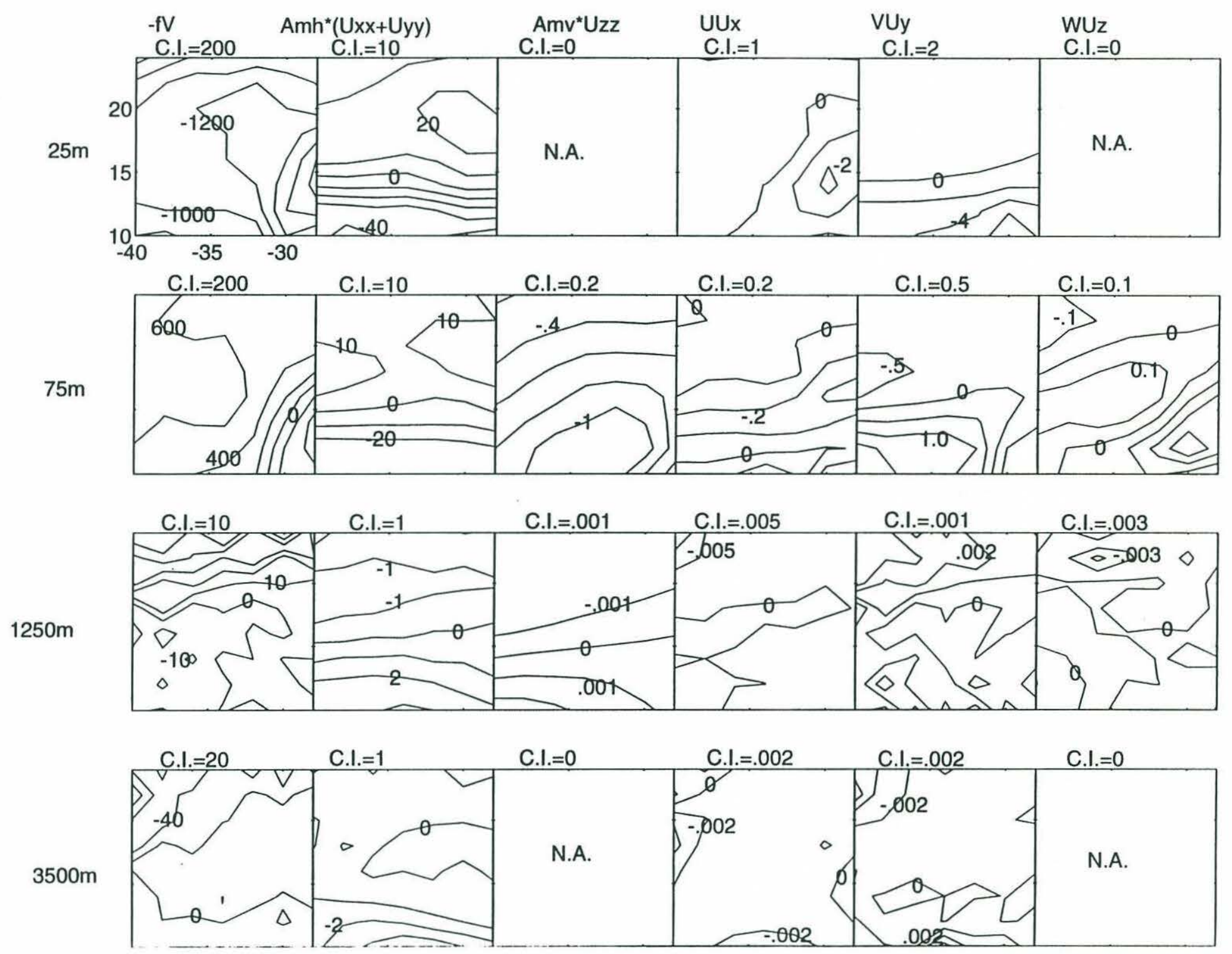

Fig. 3.3. Terms $\left(10^{-9} \mathrm{~m} / \mathrm{s}^{2}\right)$ in the zonal momentum equations at four depths (the pressure gradient term is not shown here). 

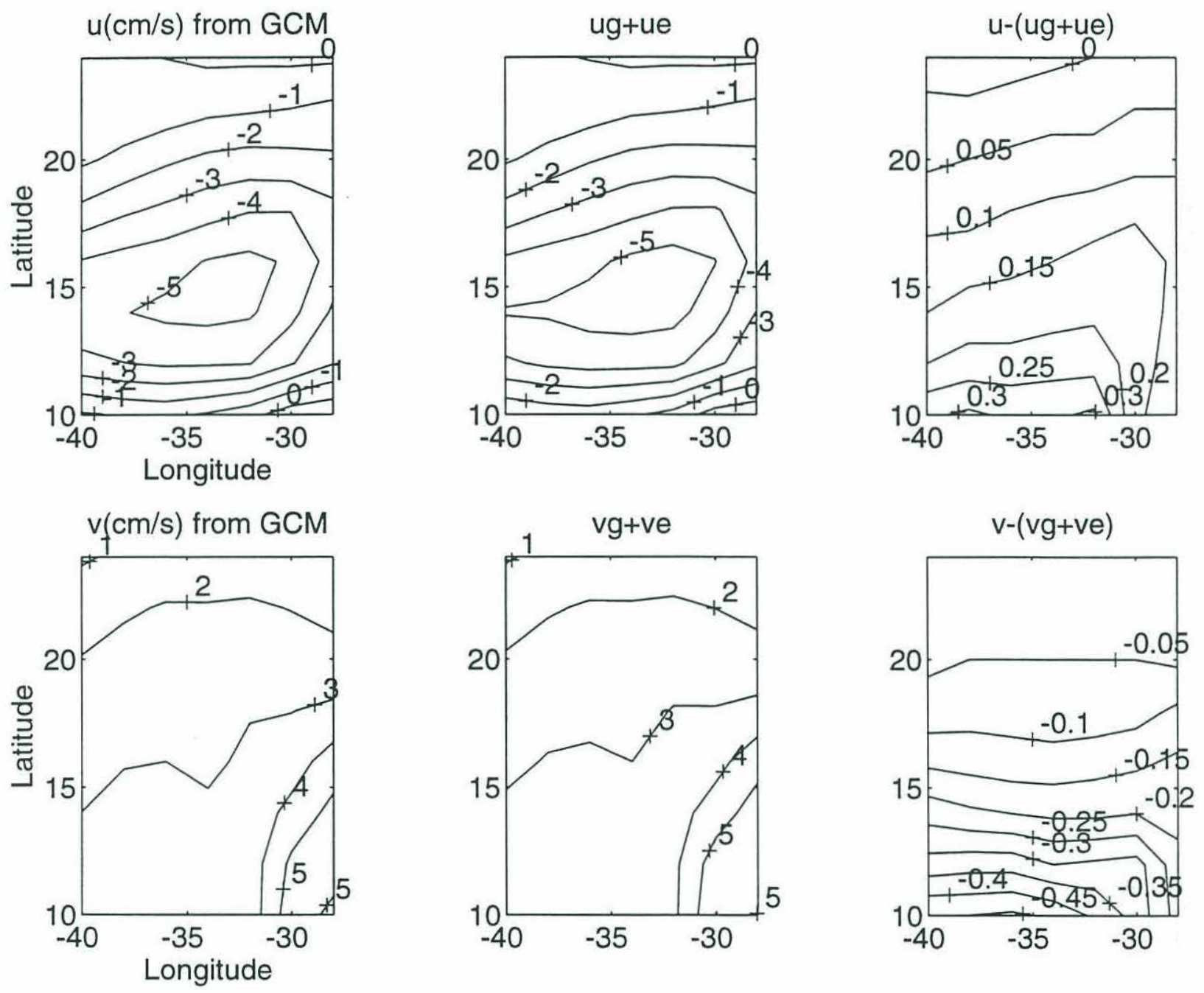

Fig. 3.4. Horizontal velocities ( $\mathrm{cm} / \mathrm{s}$ ) at 25 meter: (1) GCM absolute velocities (the 1st column); (2) sum of geostrophic and wind-driven velocities (the 2 nd column); (3) differences of columns 1 and 2 . 

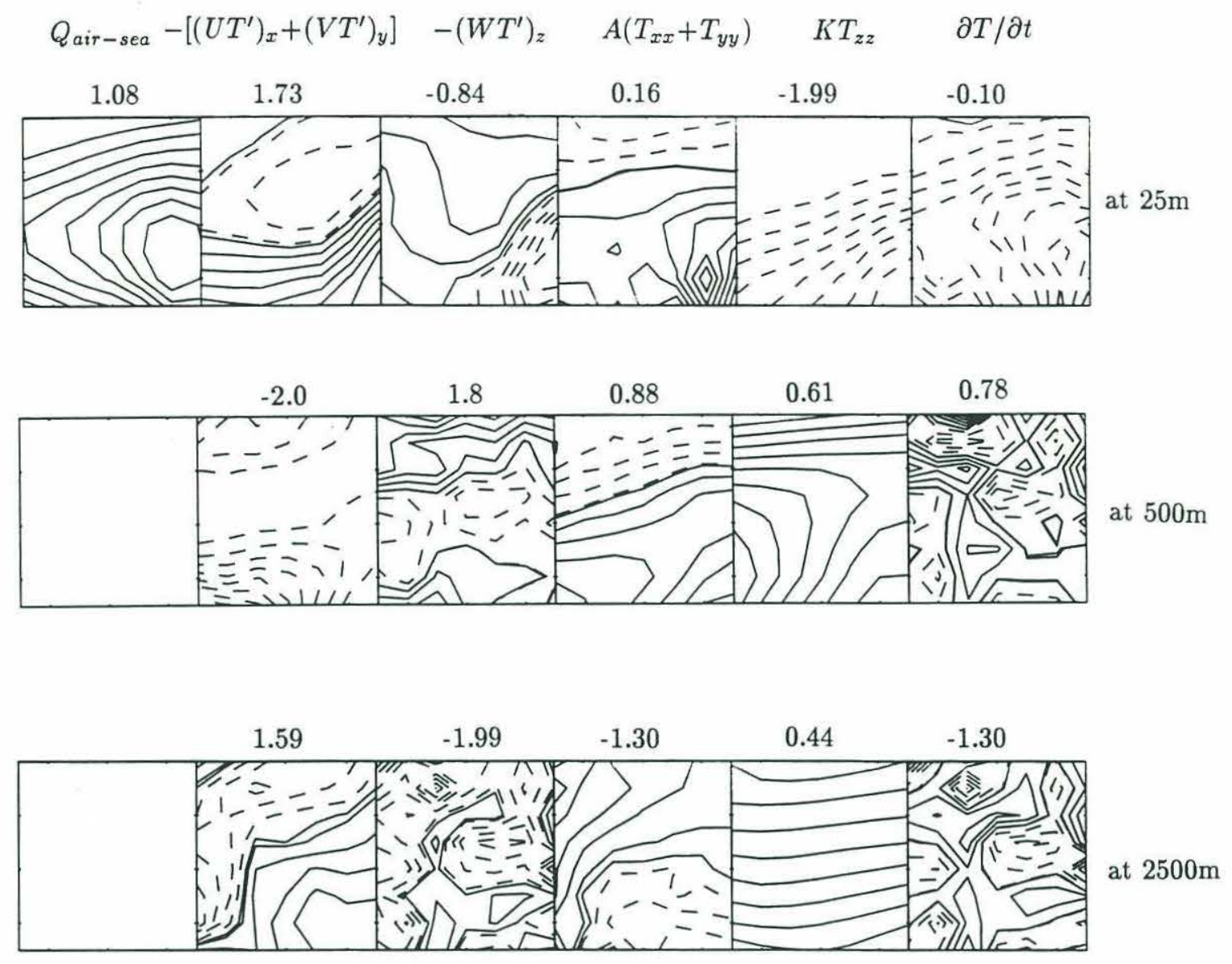

Fig. 3.5. Terms in the heat conservation equations of the non-eddy resolving GCM ocean at $25 \mathrm{~m}$, $500 \mathrm{~m}$, and $2500 \mathrm{~m}$. The numbers are the maximum or minimum contour values which indicate the magnitudes. Units are $10^{-7}, 10^{-8}$ and $10^{-10} \mathrm{~K} / \mathrm{s}$ at $25 \mathrm{~m}, 500 \mathrm{~m}$, and $2500 \mathrm{~m}$ respectively. 


\subsection{INVERSE MODEL RESULTS}

\subsubsection{Introduction}

In this section we will discuss the results of the application of the inverse model to the non-eddy resolving numerical GCM ocean. The strategy is that we will first discuss a simpler case-the upper-layer model results without the surface (frictional) layer. Then the surface layer will be incorporated in the model by using the Ekman theory and we will see how well the circulations in the surface layer and surface heat and fresh water fluxes are determined. Thirdly we will examine how the data "noise" will affect the inverse solutions by applying the inverse model to the deep-level data where the data "noises" are larger than the diffusion terms, and by allowing more freedom on the solutions for the diffusion coefficients (e.g., by allowing the horizontal diffusivity to be different constants on different vertical levels). After these experimentations on the individual aspects of the numerical ocean and the inverse model, more thorough analyses of the inverse model results in the domain of the numerical GCM data will be addressed by running the full-layer model (all 10 levels are included) and utilizing various scaling techniques. Finally, experiments on the parameterization of the diffusive parameters will be carried out with the objective of getting some guidelines for applying them to the real ocean.

The finite difference scheme and configurations of the inverse model are taken to be the same as those of the numerical GCM. The grid resolutions are $2^{\circ}$ in longitude and latitude. This choice of the grid resolutions results in grid points of $9 \times 10$ in the zonal and meridional directions respectively in the inverse model domain. With the special arrangement of the grid points for the horizontal and 
vertical velocities in the GCM scheme, the integrated vorticity equation described in Chapter 2 is actually the finite difference form of continuity equation but with less accuracy (exact geostrophic balance is used in deriving the linearized potential vorticity equation $\beta v=f w_{z}$ from the continuity equation), and it provides no further accurate information in addition to that provided by the continuity equation. Therefore the integrated vorticity constraint in the inverse model is not used in this study.

The inputs for the inverse model are the GCM fields of potential temperature and salinity. The unknown parameters to be estimated are the streamfunctions for horizontal velocities, the vertical velocities, and the horizontal and vertical turbulent diffusion coefficients. When the surface layer is included in the model, air-sea heat and fresh water (evaporation minus precipitation) fluxes are also to be estimated. The constraints of the inverse model are the dynamic equation, the continuity equation, and steady state conservation equations for heat and salt.

In the numerical GCM ocean, all the parameters to be estimated by the inverse model are also known (within the numerical roundoff error) -we call them the numerical GCM parameters. But we do not use them to constraint the inverse model solutions, instead we use them for comparison purposes. Statistical closeness of the estimated parameters to their corresponding numerical data shows the goodness of the inverse model estimation. For this comparison, the values of the GCM parameters in the inverse model domain are shown in Fig. 3.6 (circulation) and Fig. 3.7 (vertical velocity). As mentioned before, the horizontal and vertical turbulent diffusion coefficients are $A=1.00 \times 10^{7} \mathrm{~cm}^{2} / \mathrm{s}$ and $K=1.00 \mathrm{~cm}^{2} / \mathrm{s}$. 
Note that the velocity vector scales in Fig. 3.6 vary with depth. The horizontal mean velocities in the inverse model domain on the ten model levels are 4.30, $2.00,1.81,1.42,0.90,0.32,0.11,0.08,0.05$ and $0.10 \mathrm{~cm} / \mathrm{s}$ respectively. Circulations are stronger on the upper levels than on the deeper levels, except on the bottom level which are stronger than those on the two levels immediately above it (as can also be seen in Fig. 3.6).

It can be seen from Fig. 3.7a that the vertical velocities at depths greater that $400 \mathrm{~m}$ are very scattered in space. Fig. $3.7 \mathrm{~b}$ is a horizontally smoothed version of Fig. 3.7a. The smoothing is taken as a simple nine-point average with the nine points centered at the point at which the smoothed vertical velocity is to be computed (the concerned point itself plus eight more points immediately surrounding it. The inverse model domain is a small part of the GCM ocean domain). The reason for introducing these smoothed versions of the vertical velocity will become apparent later on when the inverse model results are discussed. 

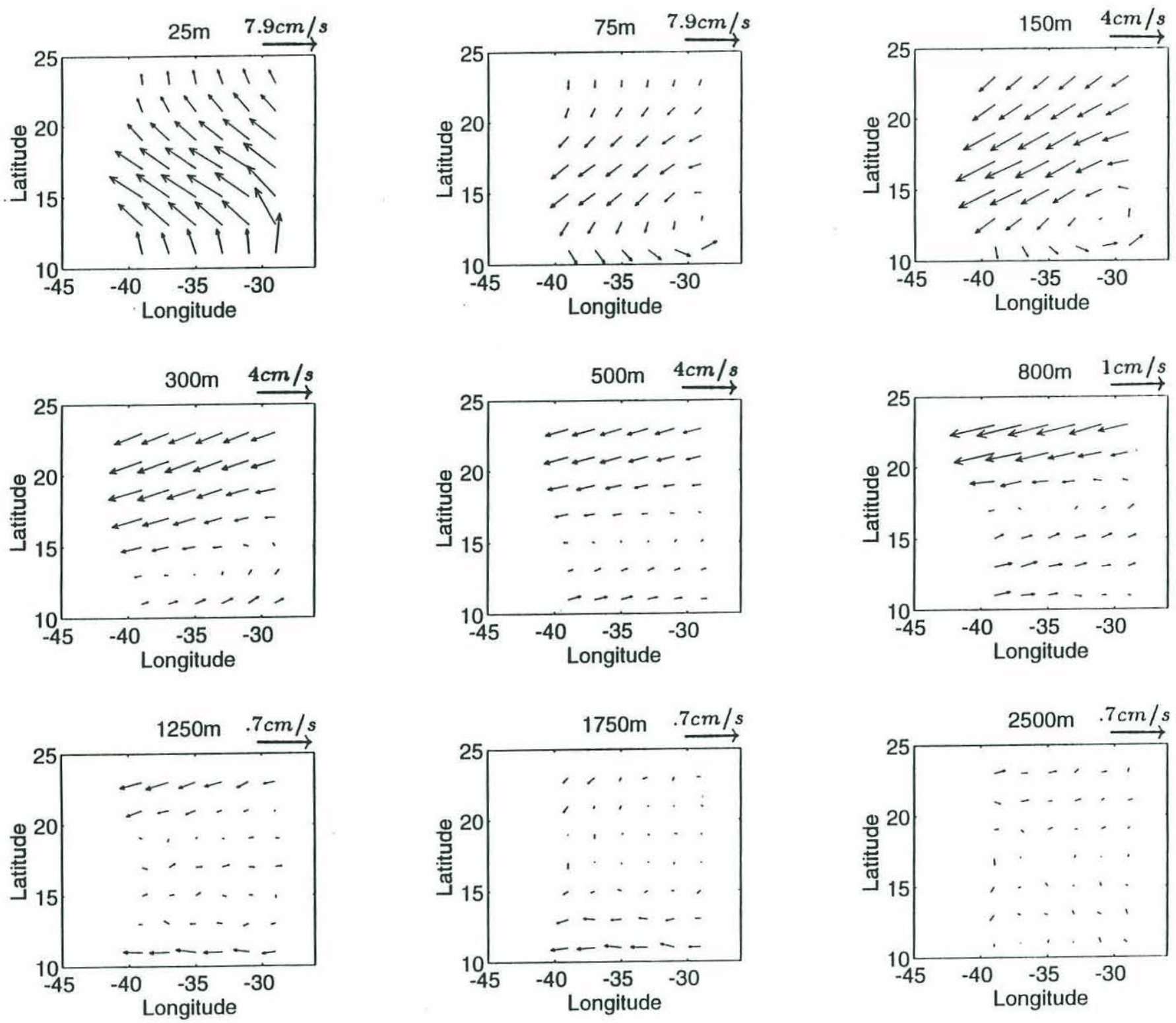

Fig. 3.6. The non-eddy resolving GCM ocean circulations at different depths. 

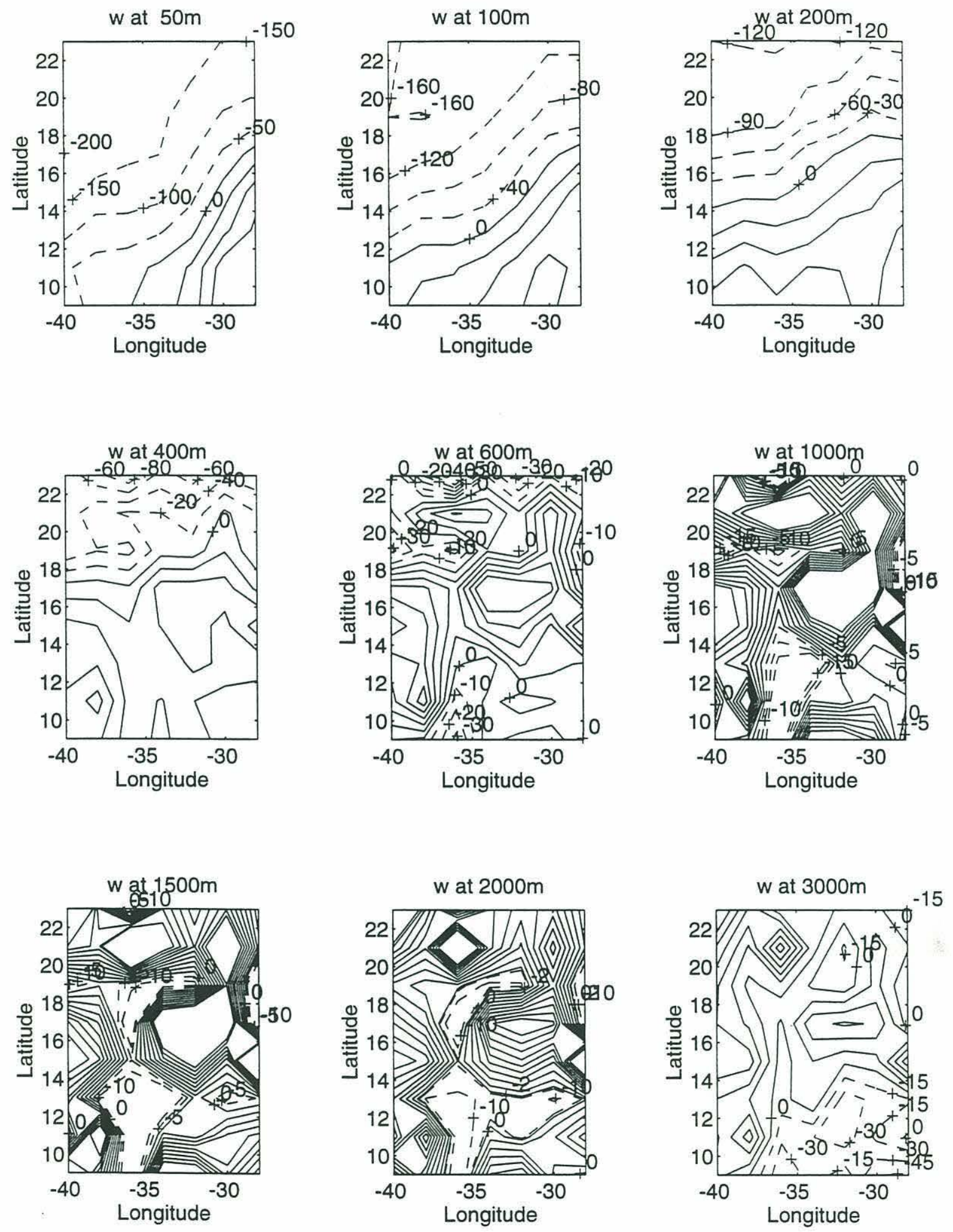

Fig. 3.7a. The non-eddy resolving GCM ocean vertical velocities $\left(10^{-6} \mathrm{~cm} / \mathrm{s}\right)$. 

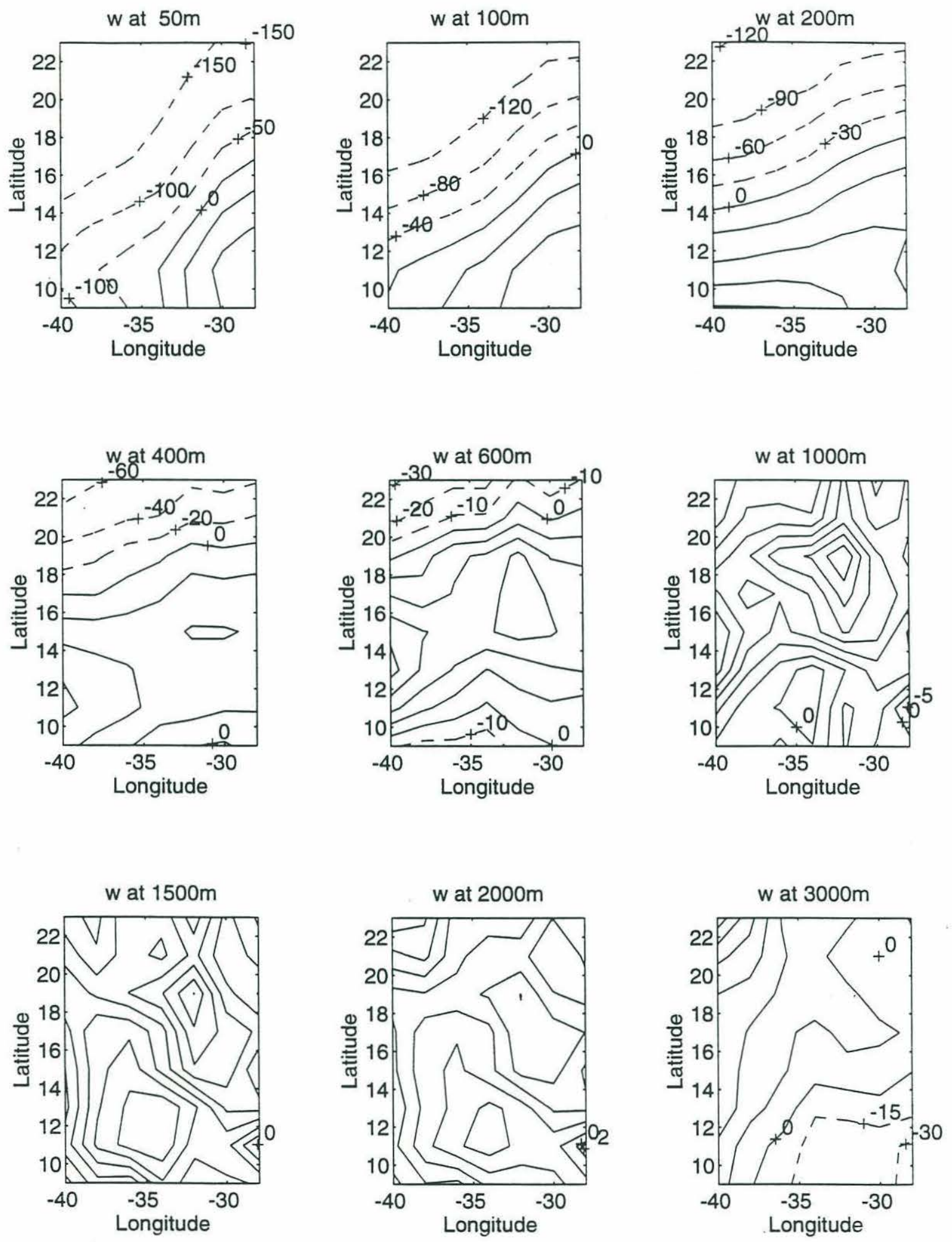

Fig. 3.7b. The 9-point horizontally smoothed GCM vertical velocities $\left(10^{-6} \mathrm{~cm} / \mathrm{s}\right)$. 


\subsubsection{Upper-Layer Model Results Without Surface Fric- tional Layer (ULM)}

As a starting point for testing the inverse model, we would like to apply it in a ocean region where the inverse model assumptions have high accuracy. As pointed out before, geostrophic balance does not hold in the surface frictional layer, and the data "noises" (the temporal variation terms) in the steady inverse model tracer conservation equations are larger than the diffusion terms at the deep levels. Therefore a logical choice for the starting approach is by running the model in the water column which excludes the levels mentioned above. In this case six levels are chosen, with the vertical cells centered at $75,150,300,500,800$, and 1250 meters. The circulation parameters are going to be estimated at these depths. The vertical interfaces of the cells, on which vertical velocities are going to be estimated, run from $50 \mathrm{~m}$ to $1500 \mathrm{~m}$. To be consistent with the numerical GCM, the horizontal diffusivity

$A$ and vertical diffusivity $K$ are taken as two unknown constants (to be estimated) in the whole domain, while the velocity parameters (the streamfunction for the horizontal velocities, and the vertical velocity) are taken as point-wise unknowns.

Equations should be row-scaled by the data noise covariance (Wunsch 1989). As mentioned above, the numerical data of temperature and salinity do not perfectly satisfy the steady conservation equation. The neglected temporal terms act like data "noise" for the inverse model. For simplicity, the conservation equations for potential temperature and salinity are scaled by depth-dependent factors: the horizontally-averaged norm of the numerical GCM temporal variation terms. This assumes that these factors at different depths are independent. In the real ocean, statistics of the data noises are usually unknown. Equation scaling without this 
information was discussed by Zhang and Hogg (1992), and examples will be further shown in section 3.3.6 and chapter 4. The relative weighting factors among the different equations (dynamic equation, conservation equations of mass, heat, and salt) are chosen experimentally so that the scaled residuals are the same magnitude for all the equations. The relative contributions of the individual equations to the solutions can be identified by the so-called data resolution (Wunsch, 1989). This data resolution indicates how the information is extracted from the equations in obtaining the solutions for the parameters.

Writing the constraint equations in the finite difference form on the $9 \times 10 \times 6$ grid points results in an equation system of 820 unknowns in 1363 equations. In order to determine how the solutions should be obtained (for example, should all the eigenvalues and eigenvectors be utilized in obtaining the solutions?), we need first to analyze the singularity of the equation system. Analysis of the singular values in Fig. 3.8a (derived from the singular value decomposition) of the coefficient matrix of this equation system as well as the Levenberg-Marquardt stabilization analysis (Fig. 3.8b) show that the equation system is overdetermined and of full rank. In Fig. 3.8b and in the equations, the estimated parameters are normalized (or nondimensionalized) by their characteristic values. These values for the streamfunction, vertical velocity, horizontal diffusivity and vertical diffusivity are chosen as $\Psi=1 \mathrm{~m}^{2} / \mathrm{s}^{2}, W=10^{-6} L_{y}^{-2} \mathrm{~m} / \mathrm{s}=2.5 \times 10^{-5} \mathrm{~m} / \mathrm{s}, A_{0}=10^{4} \mathrm{~m}^{2} / \mathrm{s}$ and $K_{0}=10^{-8} L_{y}^{-2} \mathrm{~m}^{2} / \mathrm{s}=2.5 \times 10^{-3} \mathrm{~m}^{2} / \mathrm{s}$ respectively, where $L_{y}$ is the grid distance (in meters) in the meridional direction. With these specifications, the nondimensionlized parameters have magnitude of order one $(\mathrm{O}(1))$. Effectively, this treatment is equivalent to the use of column weight factors, although the solutions 
will not be affected by column scaling if the equation system is overdetermined and of full rank. The residual norms are directly calculated from the scaled equations.
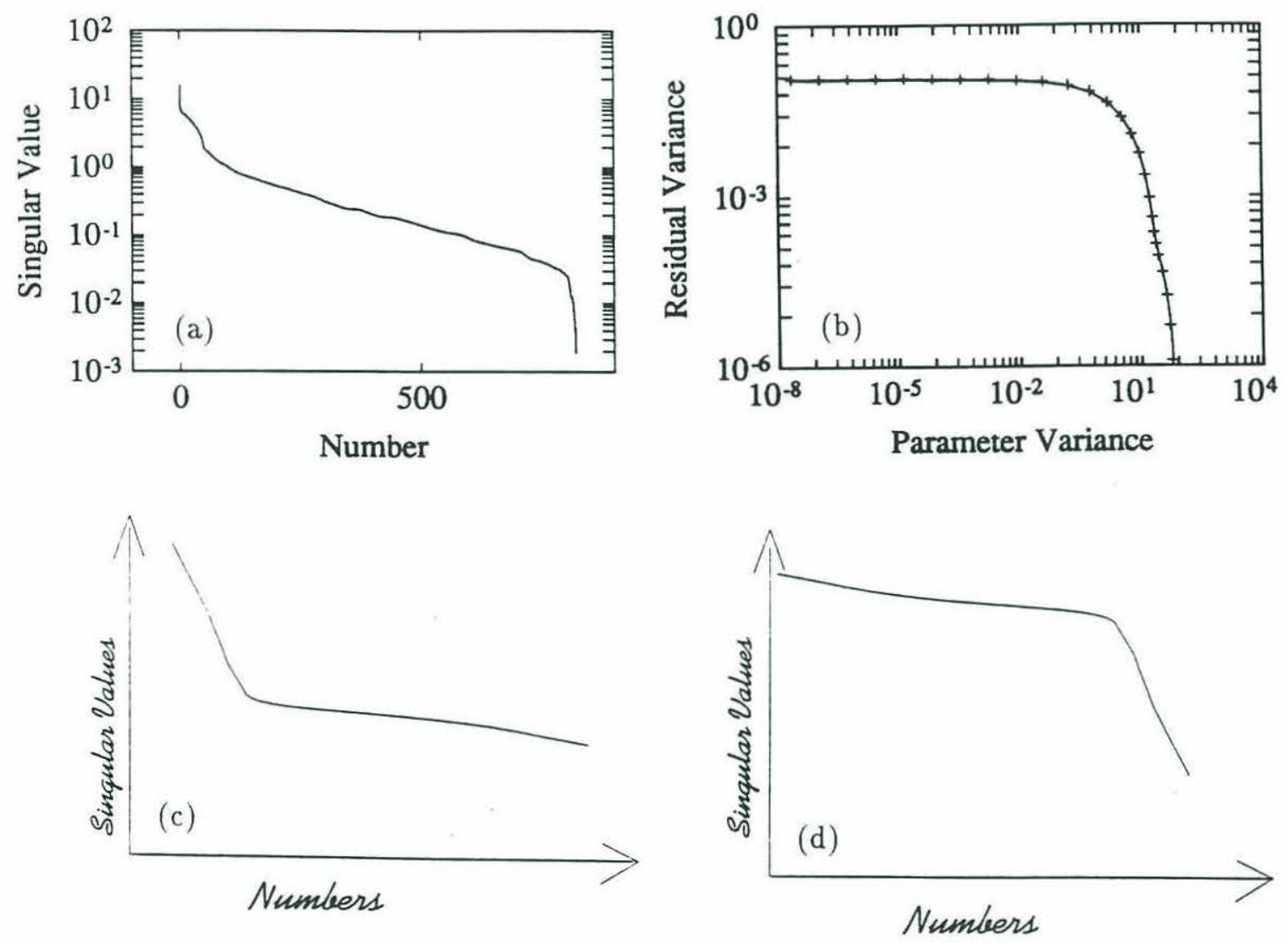

Fig. 3.8. (a) Singular value profile of the equation system in section 3.3. (b) LevenbergMarquardt stabilization diagram. (c) same as in (a) but the dynamic equation is down weighted. (d) same as in (a) but the dynamic equation is highiy weighted. 
A first glance at the singular value profile in Fig. 3.8a might lead one to believe that the system was singular because of the rapid drop of the singular values at the end of the profile. Careful analysis shows that it is not the case. Experiments showed that, due to the proper weightings of the equations, the eigenvalues (singular values) associated with the dynamic equations (the flatter segment of the profile in Fig. 3.8a) are in the (middle) range of the eigen-values associated with the conservation equations of mass, heat and salt (the two segments with large slopes at the beginning and the end of the profile). For example, when the dynamic equations were further down-weighted, their associated singular values (the flatter segment) had migrated (shifted) downward, as shown in Fig. 3.8c, and the two large-slope segments associated with the conservation equations connected with each other at the top of the profile. On the other hand, when the dynamic equations were further highly weighted, their associated singular values had migrated upward, and the two large-slope segments connected again but this time at the end of the singular value profile (Fig. 3.8d).

Just merely looking at the singular value distribution in Fig. 3.8d, one might judge that the equation system was singular, and would cut off the rapid dropping singular values at the end of the profile and their associated eigen-vectors in obtaining the inverse model solutions. From what we have learnt above, we know that this would cut off all the contributions from the conservation equations in determining the solutions. The above assertion can be easily verified by the so called Data Resolution, which signifies the contributions of the equations to the solutions (Wunsch, 1989). When the singular values at the tail in Fig. 3.8d and their associated eigen-vectors were cut off, the data resolutions of the conservation equations are zero, while those associated with the dynamic equations are unity 
(one means full contribution). In this case the solutions were only constrained by the dynamic equations, and no information in the conservation equations was used. The "barotropic" part of the absolute velocities could not be well determined, needless to say the diffusive parameters (which only appear in the the conservation equations).

In a brief summary, we cannot judge the singularity of an equation system by merely looking at the singular value distributions. An abrupt drop in the singular value profile can only be regarded as an indicator of the singularity, and its significance must be analyzed carefully with the aid of other tools. One useful technique to judge the singularity of a particular singular value is the so called Levenberg-Marquardt stabilization analysis. The general idea of this technique and its usage will be further addressed in section 3.3.4, where the drop in the singular value distribution is more profound and there is a big gap between two singular values (which strongly indicates singularity of the equation system).

The inverse model estimated turbulent diffusion coefficients are $A=(1.077 \pm$ $.011) \times 10^{7} \mathrm{~cm}^{2} / \mathrm{s}$ and $K=(1.030 \pm .005) \mathrm{cm}^{2} / \mathrm{s}$. The errors above are the estimated standard errors (deviations) for the estimated parameters. To convert them to $95 \%$ confidence intervals (deviations), a factor of 1.96 should be multiplied. This number is derived from the assumption that the population variance of the residuals is known and normally distributed (or has the student- $t$ distribution with degree of freedom of infinity). Actually the population variance is unknown and the student- $t$ distribution with degree of freedom of 542 ( = equation number - unknown number $-1=1363-820-1$ ) should be used to get this factor, which is very close to 1.96. In terms of the $95 \%$ confidence interval, the solutions for $A$ and $K$ are $A=(1.08 \pm$ 
$.02) \times 10^{7} \mathrm{~cm}^{2} / \mathrm{s}$ and $K=(1.03 \pm .01) \mathrm{cm}^{2} / \mathrm{s}$. Hereafter in this thesis, all the errors for the estimated parameters are based on the $95 \%$ confidence interval.

The estimated diffusive parameters are very close to their numerical GCM values of $1.00 \times 10^{7} \mathrm{~cm}^{2} / \mathrm{s}$ and $1.00 \mathrm{~cm}^{2} / \mathrm{s}$ for $A$ and $K$ respectively. Although the differences are small (relative errors are $8 \%$ and $3 \%$ respectively), they are significant within their expected errors. These discrepancies occur because the numerical GCM data do not satisfy the inverse model physics perfectly. For example, the neglected time dependent terms in the tracer conservation equations have the same or even greater magnitudes than the diffusion terms in the deep layers. These "noises" are not totally white noise, and the systematic offsets can cause systematic biases of the estimations for the parameters. The effects of these data "noises" on the solutions will be further studied in section 3.3.5.

The horizontal circulations estimated from the inverse model are shown in Fig. 3.9. In this figure the vector scales are chosen the same as in Fig. 3.6. Comparison of Fig. 3.9 with Fig. 3.6 shows that the estimated circulations from the inverse model have the same flow patterns as those numerical GCM circulations on all the six levels, although the numerical GCM velocities are not used to constrain the inverse model solutions. The values of the estimated velocity are also consistent with the GCM ones within the estimated errors at most of the grid points on all the levels but the deepest one at $1250 \mathrm{~m}$. At $1250 \mathrm{~m}$, although the estimated circulations have the largest uncertainties (compared to those at upper depths), the velocity values are still significantly different from the GCM ones. These significant differences are due to the fact that the circulations themselves are very weak at and below $1250 \mathrm{~m}$ (note the different vector scales used at different depths in Fig. 3.9 and Fig. 3.6). 
These significant discrepancies can also be explained by the discrepancies between the inverse model physics and the GCM ones. One is that the numerical GCM circulations do not satisfy the thermal wind relation exactly. In section 3.2 we noted that the horizontally averaged relative imbalances between the GCM absolute velocity shears and the thermal wind shears can be as large as $20 \%$ in zonal velocity and $10 \%$ in meridional velocity (Fig. 3.2 and Table 3.1). Although errors (residuals) are allowed in the dynamic equations in the inverse model, the estimated circulations satisfy the thermal wind relation more closely than the numerical GCM ones. A close look at Fig. 3.9 and Fig. 3.6 show that the deviations of the estimated velocities from the numerical GCM ones are mainly in the zonal direction. The estimated meridional velocities are generally consistent with those in the numerical GCM ones within the estimated errors, although the meridional velocities themselves are much smaller than the zonal ones (especially on the deep levels).

The estimated vertical velocities are shown in Fig. 3.10a together with their estimated errors (Fig. 3.10b). On the top four levels at 50, 100, 200 and 400 meters, the estimated vertical velocities have the same distribution patterns in space and the same magnitudes as the numerical GCM vertical velocities (Fig. 3.7a). The estimated $w$ values are consistent with the numerical GCM ones within the estimated errors at most of the grid points.

At greater depths of 600,1000 and 1500 meters, the differences in patterns between the estimates (Fig. 3.10a) and the numerical GCM values (Fig. 3.7a) become apparent, although the differences may not be significant because of the large uncertainties in the estimates. The numerical GCM vertical velocities have a random horizontal distribution, while those from the inversion are much smoother. 
Comparisons of Fig. 3.10a with Fig. 3.7b show that the inverse model solutions are closer to the spatially smoothed fields of the numerical GCM vertical velocity. At the depths of 600, 1000 and 1500 meters, the estimates of $w$ in Fig. 3.10a are closer to the GCM values in Fig. 3.7b, the nine-point smoothed version of Fig. 3.7a, and they are numerically consistent within the estimated errors. More analyses on the estimation of the vertical velocity will be found in section 3.3.5.

\subsubsection{Experiments on the Ekman Pumping Velocity Con- straint}

Ekman pumping velocity (EPV) can be computed from climatological wind stress data if it is known. If the depth of the Ekman layer is also known, the above information can be used to constrain the vertical velocities in the inverse model. In reality, determining the depth at which the EPV constraint should be applied is difficult (for example, the viscosity is usually unknown). Also, Ekman theory is not exactly satisfied in the ocean. All these uncertainties make it difficult to determine how the EPV velocity constraint should be utilized. In this section, taking the advantage of the known physics and parameter values in the GCM ocean, we will examine how different uses of the EPV constraint affect the inverse model estimations. We will see an example of how an incorrect model could lead to "incorrect" answers. 

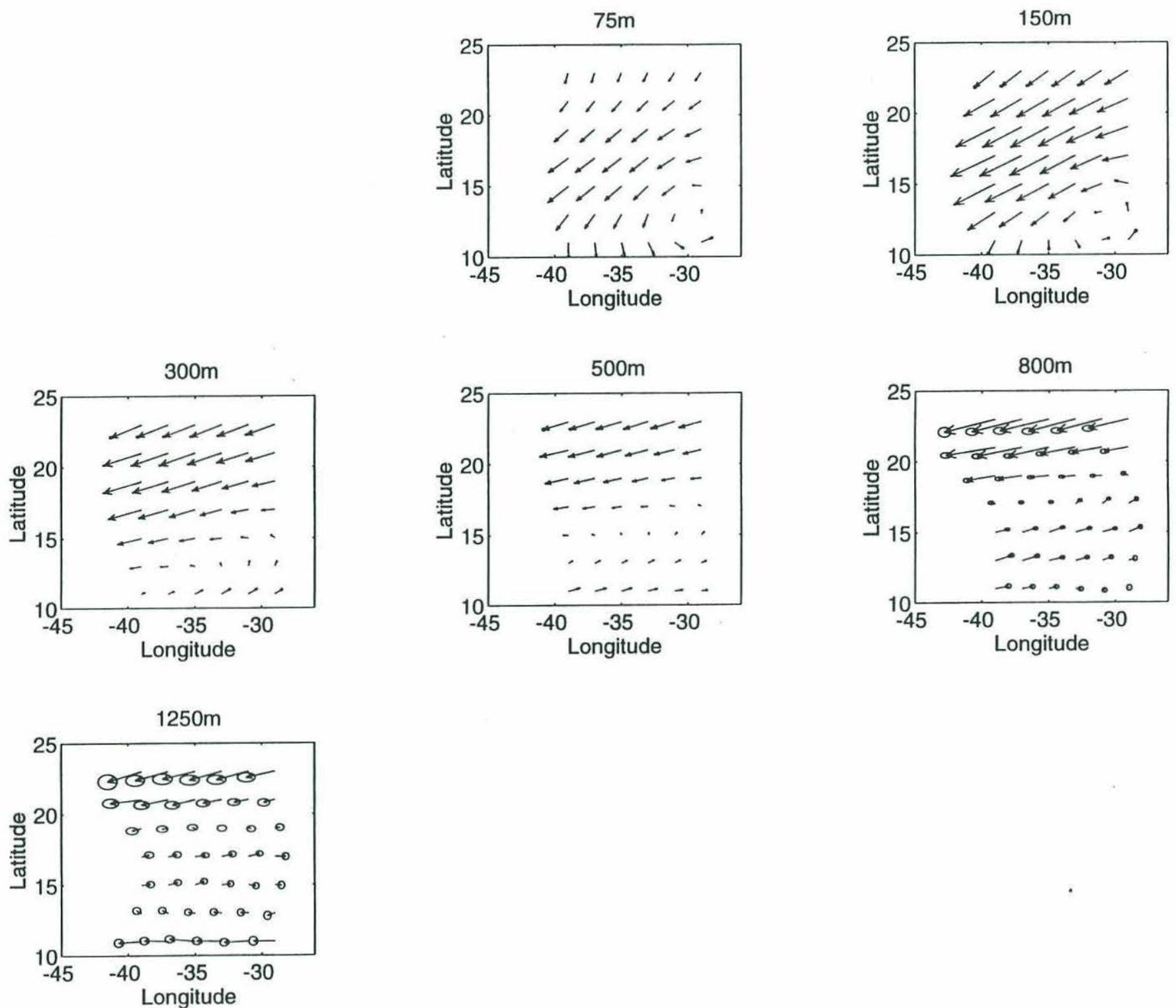

Fig. 3.9. Upper-Layer model estimated horizontal circulations with $95 \%$ confidence error ellipse.

Vector scales are the same as in Fig. 3.6. 

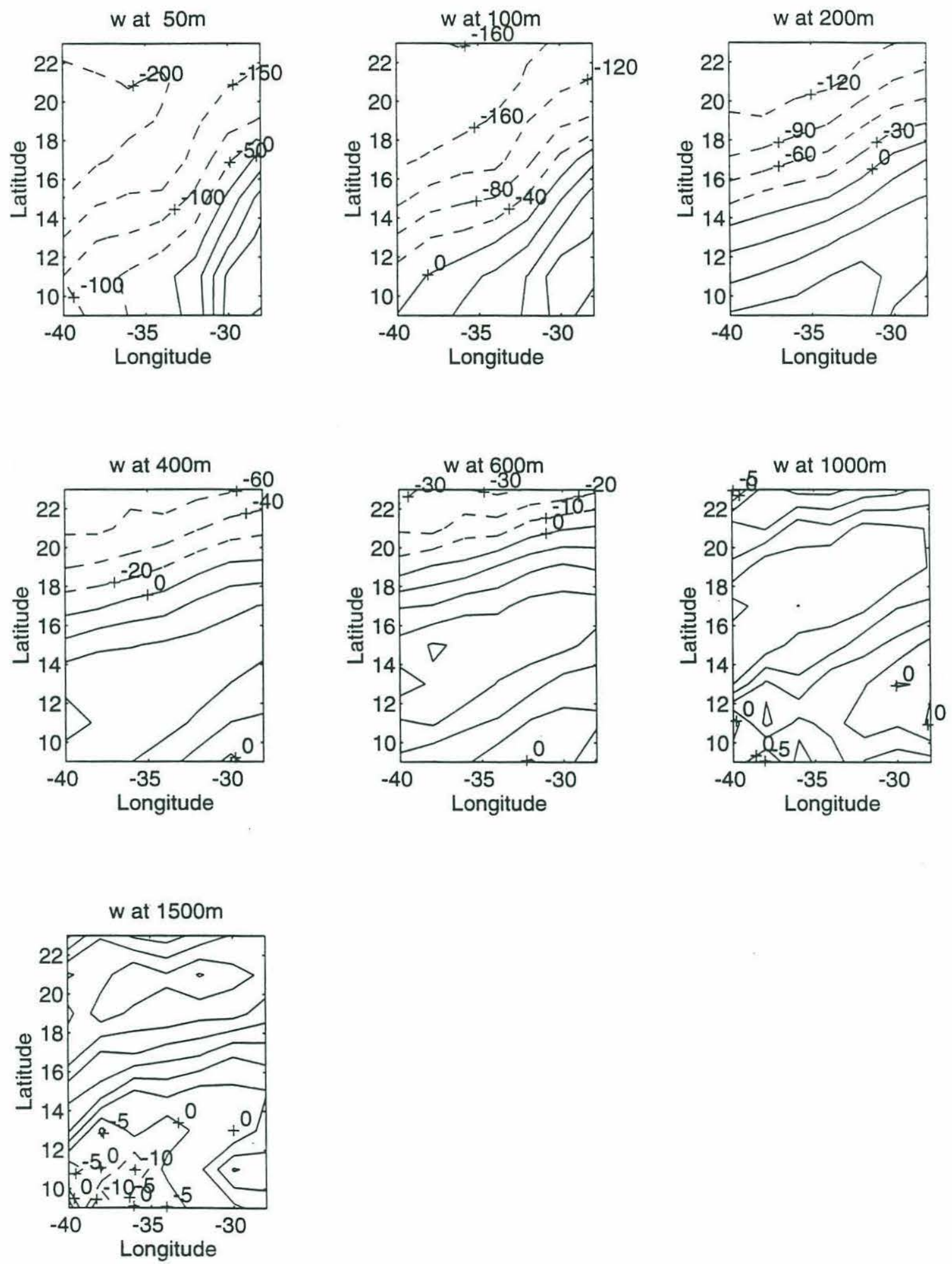

Fig. 3.10. Upper-Layer Model estimated vertical velocities (a) and their errors (b) (unit: $\left.10^{-6} \mathrm{~cm} / \mathrm{s}\right)$. 

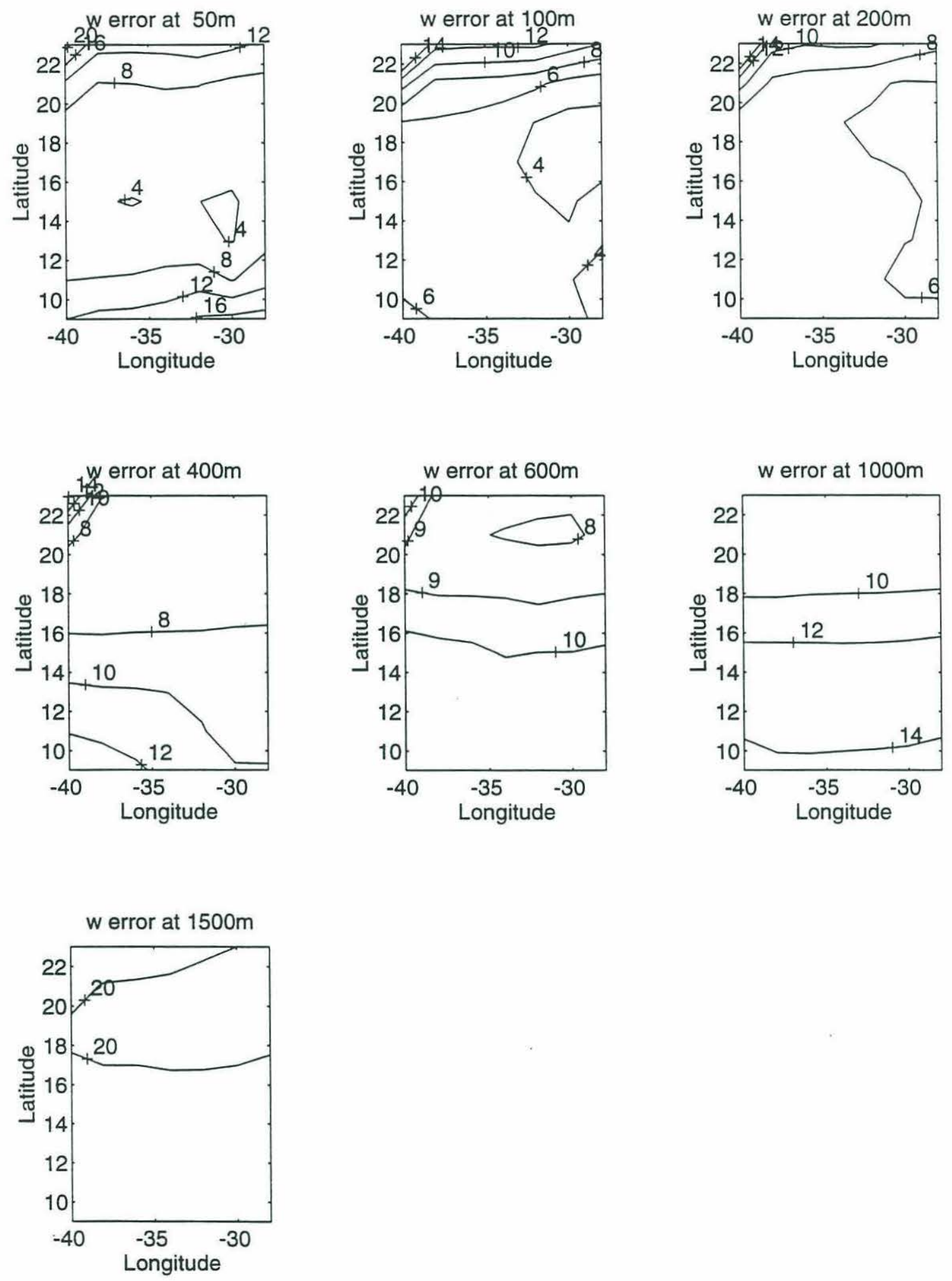

Fig. 3.10. (b) 


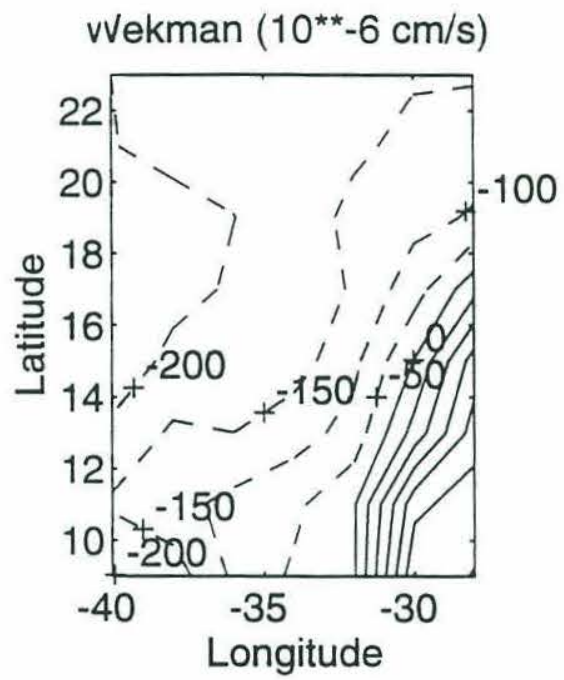

Fig. 3.11. Ekman pumping velocities computed from the wind stress of the GCM ocean.

Before considering the experiments, it is helpful to understand the differences between the Ekman pumping velocity and the actual (absolute) vertical velocity at the bottom of the surface layer. If their difference is negligible, constraining the vertical velocity by the known EPV could improve the inverse estimates. When the difference is significant, we will see how the inverse model solutions will be affected by this constraint.

The Ekman pumping velocities computed from the wind stress $\left(w_{e}=\operatorname{Curl}_{z}(\vec{\tau} / f) / \rho\right)$ in the inverse model domain are shown in Fig. 3.11. Comparisons of Fig. 11 with Fig. 3.7a and Fig. 3.10a show that the vertical velocities at the bottom of the surface mixed layer (at $50 \mathrm{~m}$ ) in the GCM ocean and the inverse model solutions (of the above section) are very similar to the EPVs. It is equally important to point out that although the horizontal patterns and magnitudes are very close to each other, 
the values are not exactly the same. It can be seen that the EPVs (Fig. 3.11) have slightly larger values than the vertical velocities estimated from the inverse model (Fig. 3.10a) and those in the GCM (Fig. 3.7a).

These differences can happen for several reasons. One is that, assuming Ekman theory is valid, the bottom of the Ekman layer may not be at the depth of $50 \mathrm{~m}$. Another possible reason for the above differences is that Ekman theory is not perfectly satisfied in the surface layer. We can do a scale analysis to illustrate this point. The momentum equations for the horizontal velocities are more complicated in the surface layer than in deep layers. As shown in section 3.2.1, nonlinear effects are minor and can be neglected in this GCM ocean. Therefore we can decompose the horizontal velocity into two major components: one is the wind-driven ocean circulation (denoted by $\vec{u}_{e}$ ), and the other is the geostrophic component of the circulations (denoted by $\vec{u}_{g}$ ). Assuming the continuity equation is accurate, the vertical velocity can be computed from the divergence of the absolute horizontal velocities:

$$
\frac{\partial w}{\partial z}=-\nabla \cdot \overrightarrow{\mathbf{u}}=-\nabla \cdot\left(\overrightarrow{\mathbf{u}}_{e}+\overrightarrow{\mathbf{u}}_{g}\right)=-\nabla \cdot \overrightarrow{\mathbf{u}}_{e}+\frac{\beta}{f} v_{g} .
$$

Integration of the above equation from the sea surface to the bottom of the surface layer yields the vertical velocity at the bottom (of $50 \mathrm{~m}$ ):

$$
w_{50 m}=w_{e}+\frac{\beta}{f} \int_{0}^{\delta_{e}} v_{g} d z
$$


where the rigid-lid assumption $(w=0)$ at the sea surface has been used. In the present case, the value of $w_{e}$ is of the order $20 \times 10^{-5} \mathrm{~cm} / \mathrm{s}$ (Fig. 3.11), and the value of $v_{g}$ is of the order $2 \mathrm{~cm} / \mathrm{s}$ at $25 \mathrm{~m}$ (from the inverse model estimations in the previous section or directly calculated from thermal wind relation, with an assumption of no flow at $2500 \mathrm{~m}$ ). With these numbers, the vertical velocity associated with the divergence of the geostrophic component of the horizontal velocity can be estimated as

$$
w_{g} \equiv \frac{\beta}{f} \int_{0}^{\delta_{e}} v_{g} d z \sim v_{g} \delta_{e} / R_{e} \tan \varphi \sim 5.8 \times 10^{-5} \mathrm{~cm} / \mathrm{s}
$$

at $15^{\circ} \mathrm{N}$, assuming the Ekman layer depth $\delta_{e}$ is of $50 \mathrm{~m}$. It can be seen that $w_{g}$ is about one-fourth of $w_{e}$, which is about the same size as the differences between the EPVs and the GCM vertical velocities at $50 \mathrm{~m}$.

In the real ocean, the Ekman pumping velocity $w_{e}$ can be computed from the distribution of the climatological wind stress. However, without knowing the "reference" velocities in advance, which is usually the case, one cannot calculate the geostrophic components of the horizontal velocities, and thus their associated vertical velocity $w_{g}$. As shown previously, the value of $w_{g}$ is usually smaller than the value of $w_{e}$. In the following experiments, we will study how the Ekman pumping velocity constraint (on the vertical velocity at the bottom of the surface layer) will affect the inverse model solutions in the case that $w_{g}$ is ignored.

The setup of the inverse model is the same as in the previous section except for the addition of one more constraint on the vertical velocities at 50-meter depth. 
The closeness of the estimated vertical velocities at $50 \mathrm{~m}$ to the EPVs $w_{e}$ can be adjusted by adjusting the weighting factors for these EPV constraints.

As mentioned before, if the covariance matrix $\mathbf{N}$ of the data noises in the equations is known, the equations should be weighted by $\mathbf{N}^{-1 / 2}$ (Wunsch, 1989). In the real ocean, information about $\mathbf{N}$ is usually unavailable. One usually estimates the noise levels for the equations in some way, and assumes that the noises are uncorrelated and the equations are down-weighted by these noise levels. Noticing that advection is the dominant process in tracer conservation equations, Zhang and Hogg (1992) argued that the conservations should be down-weighted by the typical values of the tracer gradients and the magnitudes of the flows if no other information about the data noise is available. In the previous section, the noise levels were chosen as the horizontally averaged temporal variation terms in the conservation equations. The weighting factors for the equations should be based on one's judgment of the accuracy of the equations. Larger weighting factors should be given to the more accurate equations, so that they can provide more information than other equations in obtaining the parameter solutions. The contribution of an individual equation to the solutions is shown in the data resolution (Wunsch, 1989). Equation weighting factors can be adjusted experimentally based on the above data resolution.

In the first experiment on the Ekman pumping velocity constraint, a large weight is given to the EPV constraints, which effectively forces the inverse model solutions for the vertical velocities at $50 \mathrm{~m}$ to be almost the EPVs. In this case the inverse model solutions for the vertical velocities at $50 \mathrm{~m}$ are indistinguishable from the Ekman pumping velocities, as expected, and with very small solution errors (about $2 \times 10^{-6} \mathrm{~cm} / \mathrm{s}$ uniformly in the area) (Fig. 3.12). However, the estimated 
errors for the vertical velocities on deeper levels are greatly increased compared to those in the previous section (Fig. 3.12b), due to the improper specification of the vertical velocities at the bottom of the surface layer. Within these enlarged errors, the estimated values for $w$ are not significantly different from those estimations in the previous section and those in the numerical GCM data, although the value differences are also enlarged.

The inverse model estimated values for the diffusive parameters are $A=$ $(1.43 \pm 0.07) \times 10^{7} \mathrm{~cm}^{2} / \mathrm{s}$ and $K=1.11 \pm .04 \mathrm{~cm}^{2} / \mathrm{s}$. The deviations of the estimation from the numerical GCM values are enlarged significantly. Although the values of the $w_{g}$ defined above are smaller than those of the EPV $w_{e}$, as shown before, they have significant influence on the estimations of the diffusive parameters.

The estimated horizontal circulations in this case (Fig. 3.13) are also significantly different from those estimates in the previous section and those in the numerical GCM ocean. The differences are not only in values on all the six levels, but also in patterns on the deepest two levels at $800 \mathrm{~m}$ and $1250 \mathrm{~m}$. The estimated circulation errors are greatly increased. Even so, the biases of the solutions from the numerical GCM data are still significant. These results show that incorrect specifications of the vertical velocities at the bottom of the surface layer could lead to significant biases of the solutions from their "true" values. 

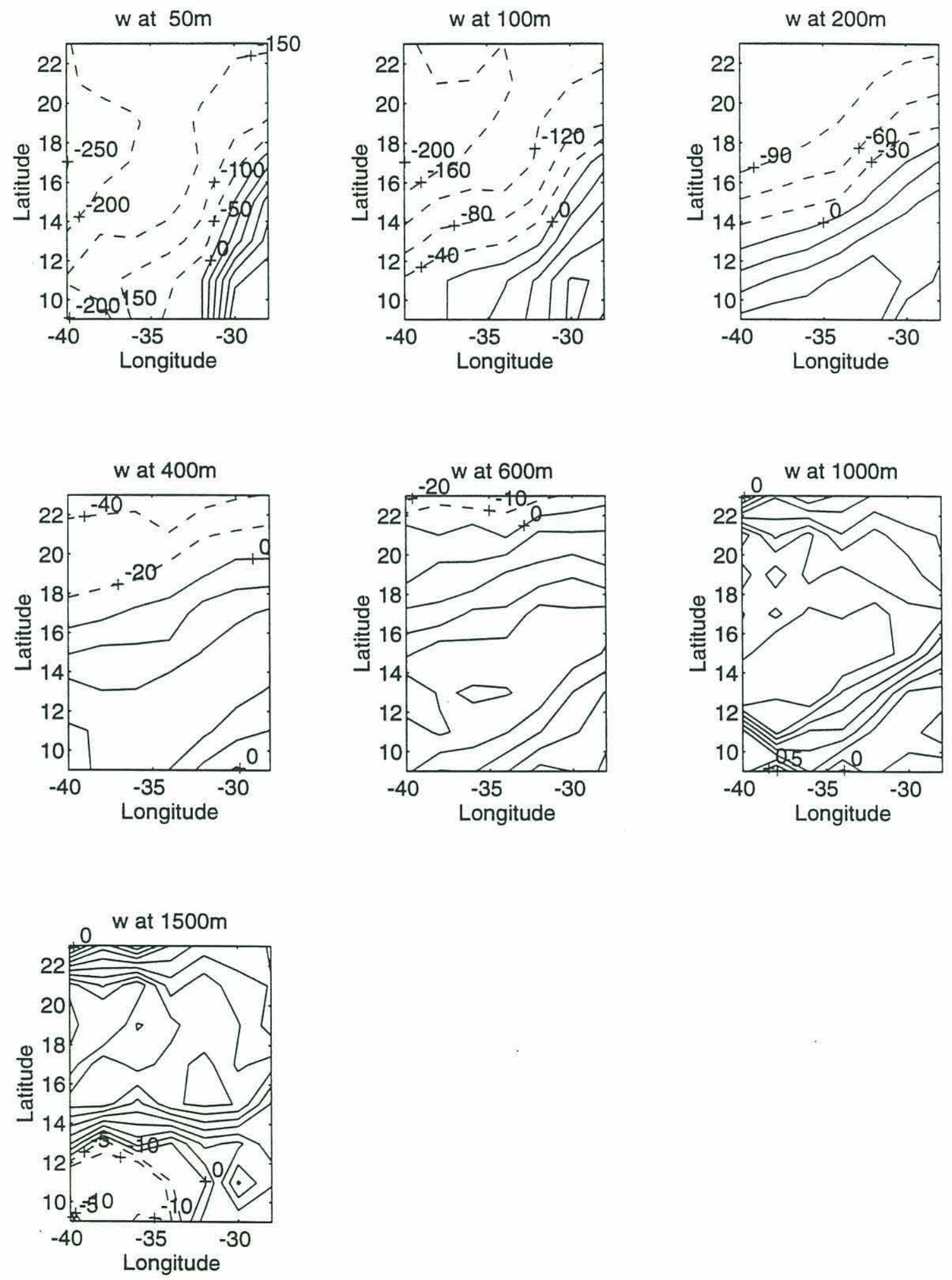

Fig. 3.12. Estimated vertical velocities (a) and their expected errors (b) from the experiment of Ekman pumping velocity constraint. The units are $10^{-\hat{o}} \mathrm{~cm} / \mathrm{s}$. 

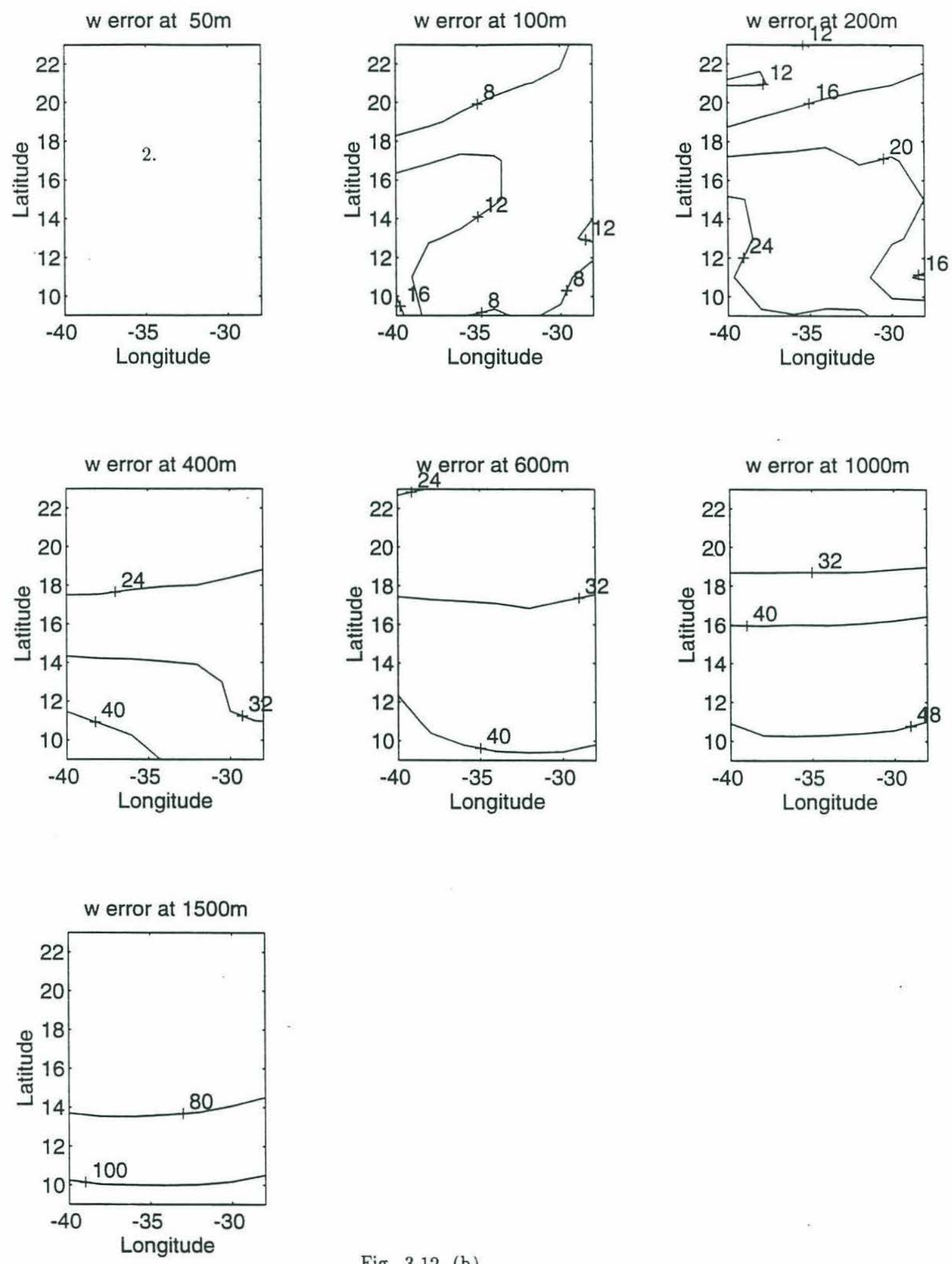

Fig. 3.12. (b) 

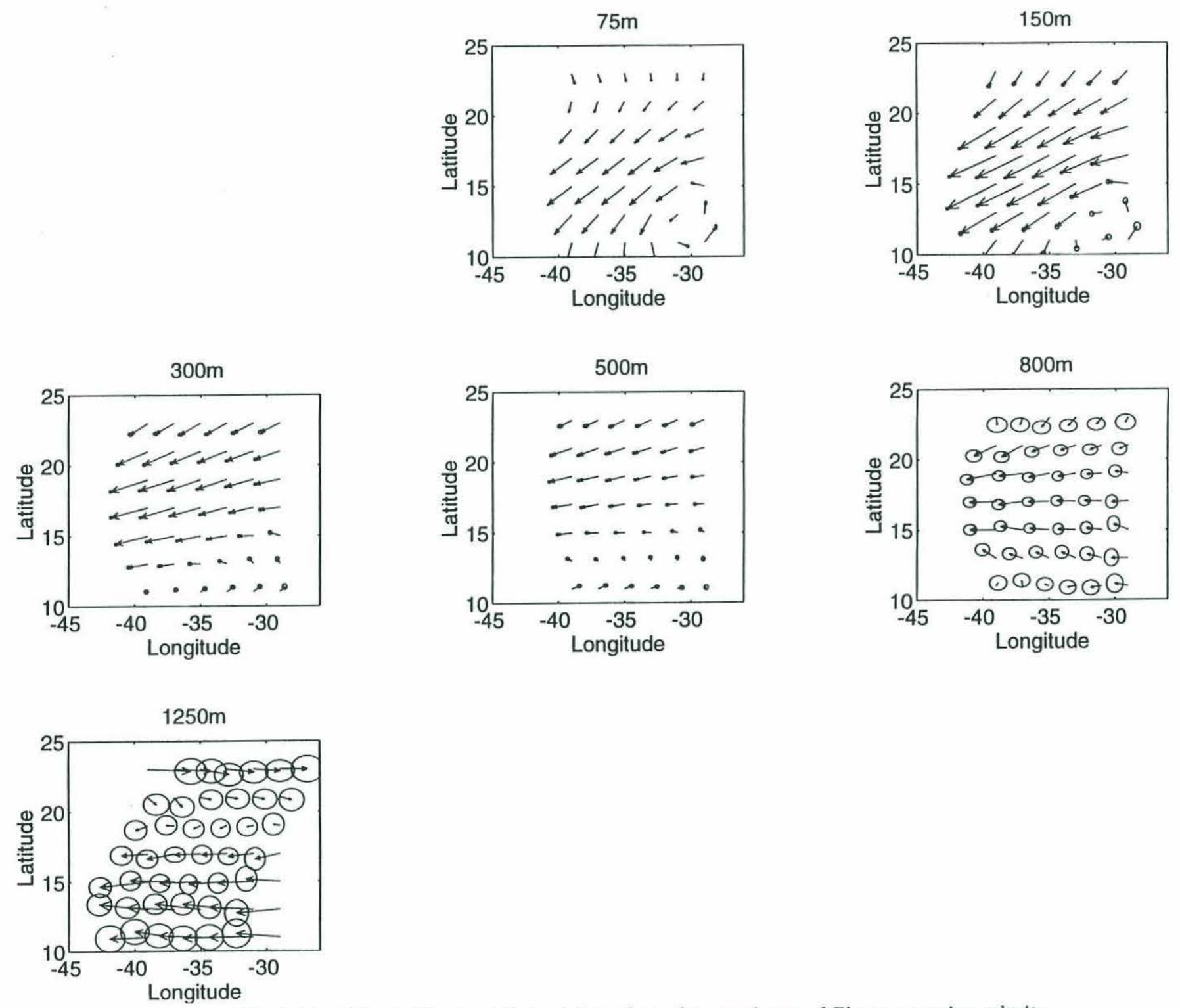

Fig. 3.13. Estimated horizontal circulations from the experiment of Ekman pumping velocity constraint. Vector scales are the same as in Fig. 3.6. 
If $\hat{X}$ is the inverse model solution (by Singular Value Decomposition, or SVD) for the true $X$ (of the equation in matrix form of $A X \approx Y$ ), and $\hat{Y}=A \hat{X}$, one can find (e.g., Wunsch, 1978) that $\hat{Y}=U U^{T} Y$, where $Y$ is the true value of $\hat{Y}$, and $U$ is the eigenvector matrix in the SVD of the coefficient matrix $A: A=U \Lambda V^{T}$. The diagonal elements of $U U^{T}$ compose the elements of the so-called data resolution. If a diagonal element of $U U^{T}$ is unity or near so, the corresponding equation contributes fully and independently to the solutions. If it is near zero, the equation has little effects on the solutions.

Comparison of the data resolution in the above case (Fig. 3.14b) with that in the previous one (Fig. 3.14a) shows that adding the Ekman pumping velocity constraint efficiently reduces the contributions of the top layer dynamic equations, continuity equations, as well as the conservation equations. Post-solution equation residuals are also increased in this case over the case without the Ekman pumping velocity constraint. This means that forcing the vertical velocities at $50 \mathrm{~m}$ to be the EPVs makes the satisfaction of the other constraint equations more difficult-even with the significant deviations of the parameter solutions from their true values, the residuals in the equations cannot be further reduced. This example shows how an incorrect model could lead to "incorrect" parameter estimates.

In the second experiment, the EPV constraints are greatly down-weighted (the scaling factor is $1 \%$ of the one used in the previous experiment). Due to the nature of the overdeterminacy of the equation system even without the EPV constraints, this experiment simply recovers the parameters estimated in the previous section where no EPV constraints were used. The Ekman pumping velocity constraints have very limited effects. 
In summary, the above two experiments show that unless one has strong reawon to believe that the vertical velocity at a certain depth should have prescribed values (e.g., the Ekman pumping velocity), one should use these constraints very (arefully. Generally, small weighting factors should be given to these constraints. If the system without these constraints has enough information to determine the parameter solutions, these constraints have little effect on the inverse model solutions. ()n the other hand, if the information for determining the parameter solutions is insufficient. information will be extracted more effectively from these constraints and contribute to the solutions (see next section for determining the air-sea fluxes).
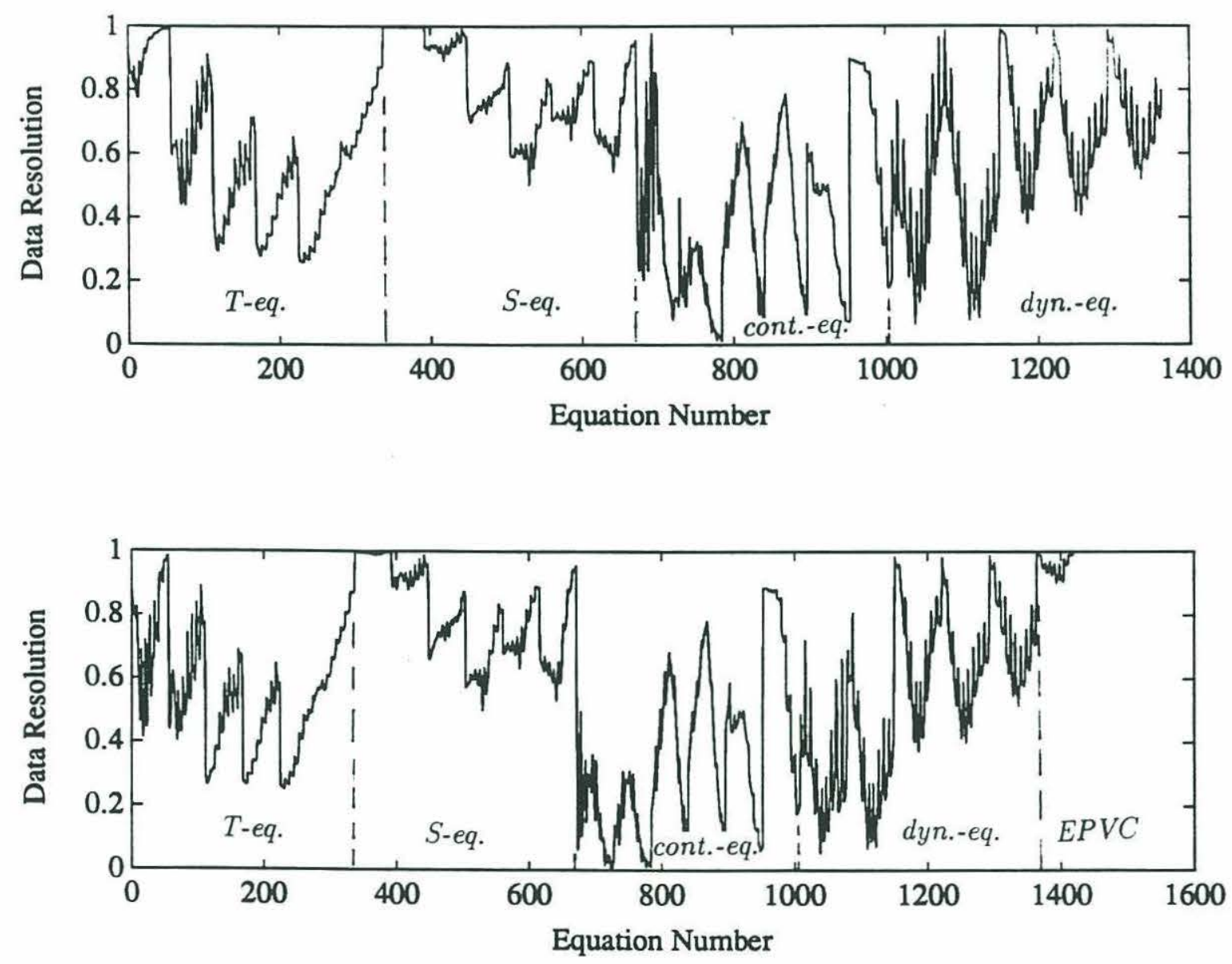

Fig. 3.14. Data resolutions: (a) the Lipper-Layer Model: (b) the Upper-Layer Model with Ekman pumping velocity constraint. 


\subsubsection{Model with Frictional Surface Layer-Determine the Air-Sea Fluxes}

As mentioned before, geostrophy does not hold in the frictional surface layer (here from 0 to $50 \mathrm{~m}$ ), as it is under direct influence of wind stress. There are also property exchanges between the ocean and the atmosphere (e.g., heat and fresh water fluxes). Therefore more terms should be included in the inverse model equations in this layer.

If the absolute velocity below the surface layer has been determined (by the deeper levels, as in previous sections), then the geostrophic part of the absolute velocity in the surface layer (at $25 \mathrm{~m}$ ) can be computed through the thermal wind relation. The horizontal Ekman transport (thus its divergence) over the whole surface layer can also be calculated from the wind stress $\left(\overrightarrow{\mathbf{M}}_{e}=\hat{k} \times \vec{\tau} /(\rho f)\right)$. Neglecting the nonlinear effects, the sum of the above two parts can be regarded as the total horizontal mass transport. Providing that the horizontal and vertical diffusion coefficients for water properties $(A, K)$ and the vertical velocity $w$ at the bottom of the surface layer $(50 \mathrm{~m}$ ) have been determined (by the deeper levels, as in previous sections), the conservations of mass, heat, and salt can be used to estimate the surface air-sea heat and fresh water fluxes. This surface layer was formulated in section 2.3.3.

The above processes are realized by incorporating the surface layer equations into the inverse model, but they are greatly down-weighted by a factor, say, of 0.01 . As long as this factor is small, it will be shown that the inverse model results are not sensitive to the values of this factor. The small weighting factor was chosen because that the physics in the surface layer of the inverse model is much simpler 
than that of the GCM ocean, and thus the equation noise levels are assumed to be larger in this layer than in deeper layers. Also, there are more unknowns, namely the air-sea heat and fresh water fluxes, to be determined in this layer than in deeper layers, but the constraint equations are the same.

With the above assumption, the mass conservation equation in the surface layer is in the form of

$$
\nabla \cdot \overrightarrow{\mathbf{u}}_{g}+\frac{\partial w}{\partial z}+\frac{1}{\delta_{e}} \nabla \cdot \overrightarrow{\mathbf{M}}_{e}=0
$$

(Here the Ekman layer thickness $\delta_{e}$ is taken as $50 \mathrm{~m}$.) As this equation is also highly down-weighted, the solutions for the geostrophic velocity component $\left(\overrightarrow{\mathbf{u}}_{g}\right)$ and the vertical velocity $w$ in this layer are mainly determined by deep-layer equations and the thermal wind relation. Since $\nabla \cdot \vec{M}_{e}$ is also prescribed by wind stress, the above equation can be used for diagnostic purposes.

With these configurations, the singular values of the coefficient matrix of the equation system are shown in Fig. 3.15a. An immediate impression of looking at this figure is the segmentation of the singular value profile-there is a rapid drop in singular value at $4.6 \times 10^{-3}$ and one might think that the equation system is singular. But more detailed analysis shows that this is not the case. The singular values at the tail of the profile are associated with the equations in the surface layer. The big gap between these singular values and the singular values above (associated with the equations of the deep layers) is caused by the fact that the equations in the surface layer are greatly down-weighted (a factor of 0.01 ). In obtaining the inverse model solutions, cutting off the contributions of these "small" singular values and their corresponding eigenvectors would be equivalent to cutting off the contributions of the surface layer equations, and therefore we would not expect to get "realistic" solutions for the surface heat and fresh water fluxes. 

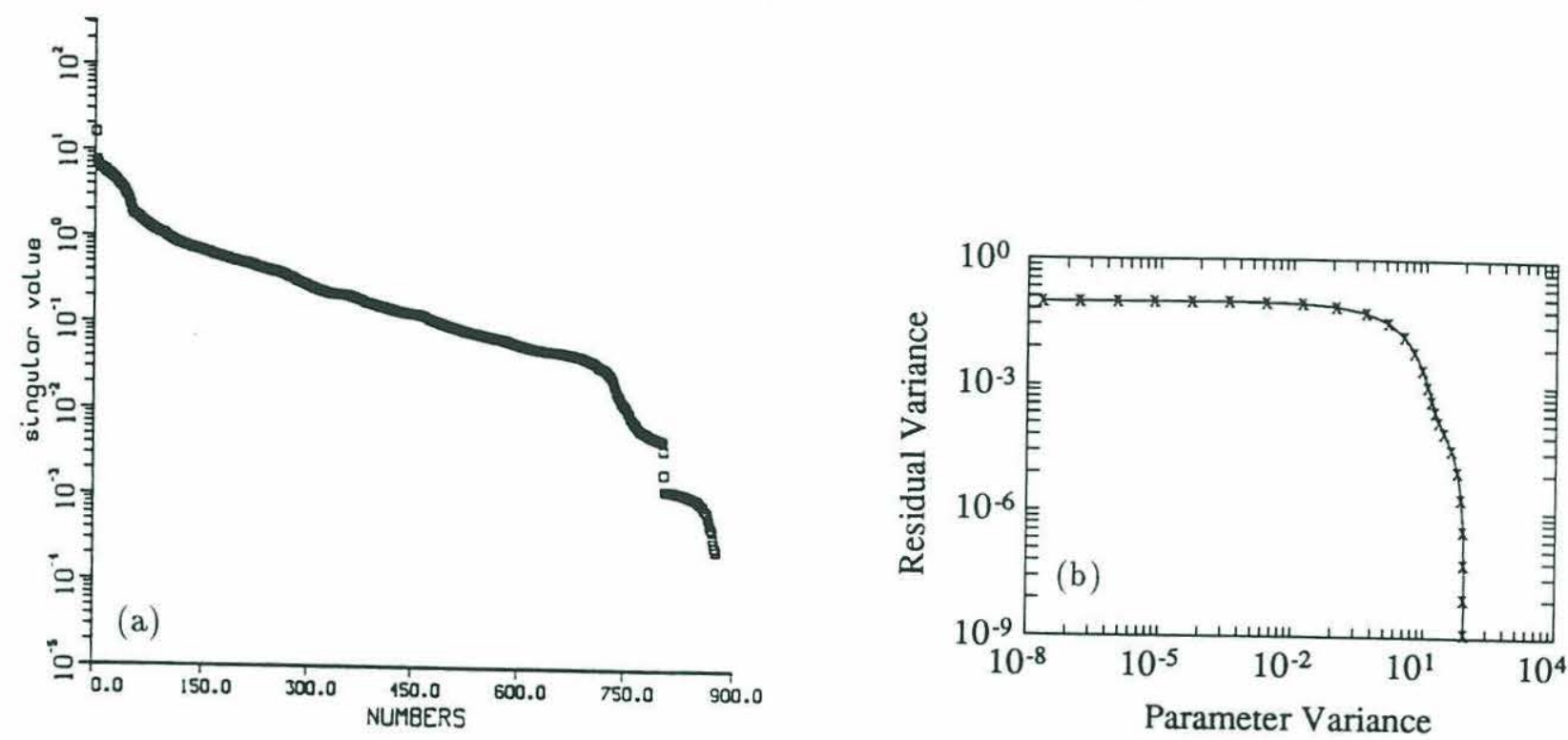

Fig. 3.15. Singular value profile (a) and the Levenberg-Marquardt stabilization diagram (b) for the Upper-Layer Model with frictional surface laver.

The non-singularity of the equation system is also shown in the corresponding Levenberg-Marquardt stabilization diagram (Fig. 3.15b). Basically the LevenbergMarquardt stabilization technique is used to judge whether small singular values contribute significantly to a reduction in residual variance without inordinate increases in parameter variance (e.g., Lawson and Hanson, 1974). Plotted in Fig. $3.15 \mathrm{~b}$ are the residual variance versus the parameter variance as a function of the "Levenberg-Marquardt stabilization" parameter $\lambda$. Without this parameter (or $\lambda=0$ ), the inverse model solutions are obtained by minimizing the residual norm of the equations $\|A * X-B\|$. Obtained in this way, it can be shown that the parameter solutions themselves are proportional to $1 / s_{i}$ while the estimated errors for the parameters are proportional to $1 / s_{i}^{2}$, where the $s_{i}$ 's are the singular values of the equation system. If the equation system is singular, the parameter variance 
grows very rapidly as the singular values decrease abruptly. The estimated errors have even greater growth rate. In these cases the parameter variance is usually unreasonably large and the solutions have no significance (because of the even larger estimated errors).

In order to obtain significant solutions in reasonable magnitudes, the objective function to be minimized can be chosen as a combination of the residual norm and solution norm in the form of

$$
J=\|A * X-B\|^{2}+\lambda^{2}\|X\|^{2},
$$

which is known as tapered least squares. In this formulation, it can be shown that the solution norm is proportional to $s_{i} /\left(s_{i}^{2}+\lambda^{2}\right)^{1 / 2}$, and the estimated errors are proportional to $1 /\left(s_{i}^{2}+\lambda^{2}\right)$. From the above relations we can see that the parameter $\lambda$ tapers off the contributions of the singular values which are smaller than the value of $\lambda$. The growth of the solution norm and the errors are stabilized by this parameter in the case of singularity. The trade-off between the residual norm and the solution norm is determined by the choice of $\lambda$ (It can be shown that the solution norm square is proportional to $\left[s_{i} /\left(s_{i}^{2}+\lambda^{2}\right)\right]^{2}$ and the residual norm square is proportional to $\left.\left[\lambda^{2} /\left(s_{i}^{2}+\lambda^{2}\right)\right]^{2}\right)$.

Shown in Fig. 3.15b is the residual variance v.s. the parameter variance as a function of the Levenberg-Marquardt stabilization parameter $\lambda$. The largest value of $\lambda$ (the top-most $\mathrm{x}$ point) was chosen as the largest singular value, and the smallest (the bottom $\mathrm{x}$ point) was chosen as $1 / 10^{1 / 2}$ of the smallest singular value. It can be seen from this figure that as $\lambda$ decreases, first the parameter variance increases very rapidly without much decrease in the residual variance, then the residual variance decreases very rapidly without much increase in the 
parameter variance. This implies that including the contributions of all the singular values and the corresponding eigenvectors does not increase the parameter variance significantly while the residual variance is greatly reduced. On the other hand, if one thought that the equation system was singular from looking at the singular value profile in Fig. 3.15a, one would cut off the contributions of the singular values in the tail of the profile in Fig. 3.15a (all singular values smaller than $4.6 \times 10^{-3}$ ). The parameter variance would decrease from 85 to 41 , but at the cost of increasing the residual variance from $1.2 \times 10^{-9}$ to $3.1 \times 10^{-5}$. One would not make this trade-off between the solution variance and the residual variance unless there is a reason to tolerate large residual variance.

As the equations in the surface layer are highly down-weighted, it is shown that, as expected, the inverse model solutions for the parameters of diffusion coefficients, vertical velocity, and the horizontal circulations on the deep levels (below the surface layer) are about the same as those in section 3.3.2, where the surface layer was excluded. For example, the estimated diffusion coefficients are $A=(1.08 \pm 0.04) \times 10^{7} \mathrm{~cm}^{2} / \mathrm{s}$ and $K=(1.03 \pm .02) \mathrm{cm}^{2} / \mathrm{s}$. These solutions are the same as in section 3.3.2, but with slightly larger errors in the present case. This is also true for the horizontal circulations and vertical velocities.

In the surface layer, the geostrophic components of the horizontal circulations were treated as unknowns and are estimated from the inverse model. The total horizontal velocities at $25 \mathrm{~m}$ can be calculated as $\overrightarrow{\mathbf{u}}_{g}+\frac{1}{\delta_{e}} \overrightarrow{\mathbf{M}}_{e}$, where $\overrightarrow{\mathbf{M}}_{e}$ is the Ekman transport and $\delta_{e}$ the Ekman layer depth taken as $50 \mathrm{~m}$ in this case. The results are shown in Fig. 3.16. It can be seen from this figure that the estimated 
circulations are very similar to the numerical GCM circulations at the depth of 25 m. In fact, they are consistent with each other within the estimated errors.

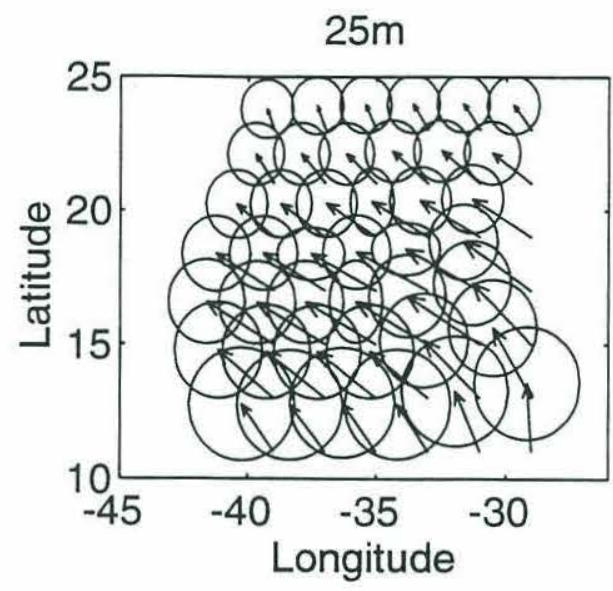

Fig. 3.16. Estimated absolute horizontal velocities with their expected error ellipses at $25 \mathrm{~m}$ from the Upper-Layer Model with frictional surface layer. Vector scale is the same as in Fig. 3.6.

The post-inverse residuals in the continuity equation in the surface layer are small, and this justifies the assumptions we used in deriving the surface layer inverse model.

The estimated air-sea surface heat and fresh water fluxes from the inverse model are shown in Fig. 3.17. Also shown in this figure are the heat and fresh water fluxes used in the numerical GCM. Comparisons show that the patterns of the inverse model estimates are very similar to those of the numerical GCM data in the whole domain. Numerically, the estimated values for these parameters are also nicely consistent with the numerical GCM data within the estimated error bars.

Compared to the solutions in the deep layers, the estimated parameters in the surface layer have much larger solution errors (see Fig. 3.16 and Fig. 3.17). These large errors are caused by the fact that there are more unknowns to be estimated 
in this layer than in the deep layers, while there is no increase in constraints. The lack of more information in determining the solutions results in larger solution uncertainties. With these large uncertainties, the inverse model solutions are easily consistent with the numerical GCM data within the solution errors. But it should be emphasized that regardless of the solution errors, the inverse model solutions themselves are very close to those values of numerical GCM parameters. 

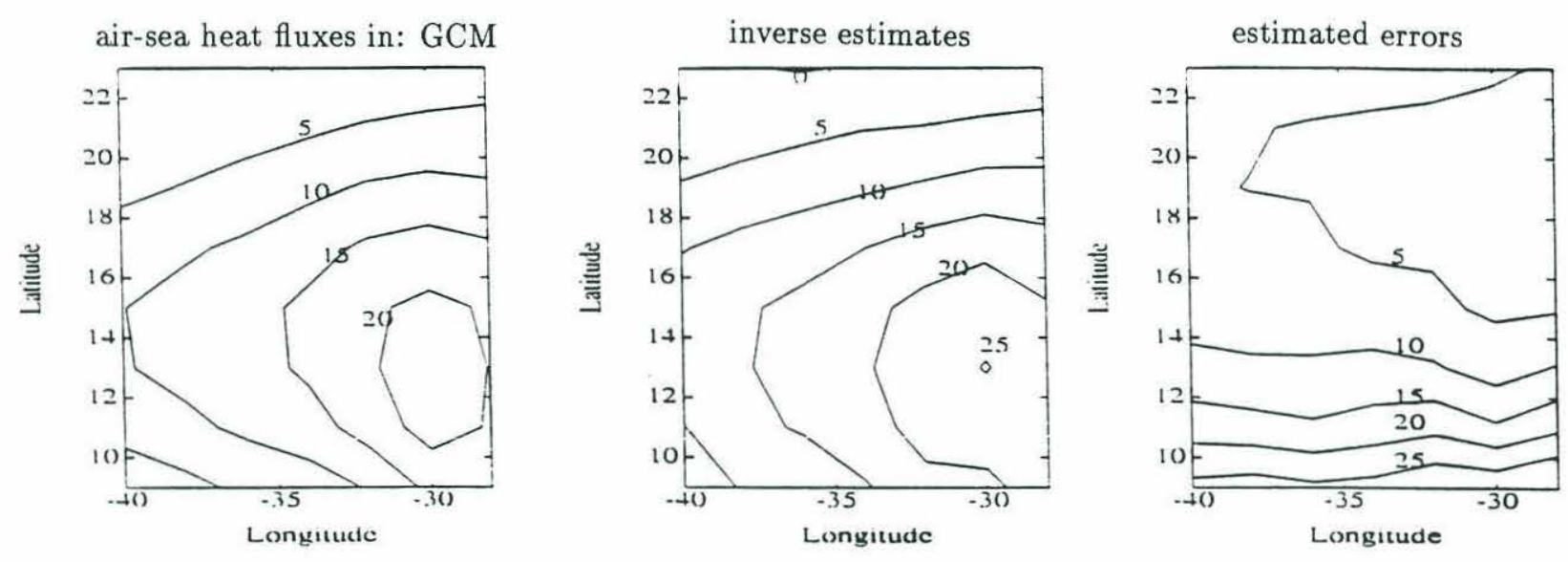

The EMP fluxes in: GCM
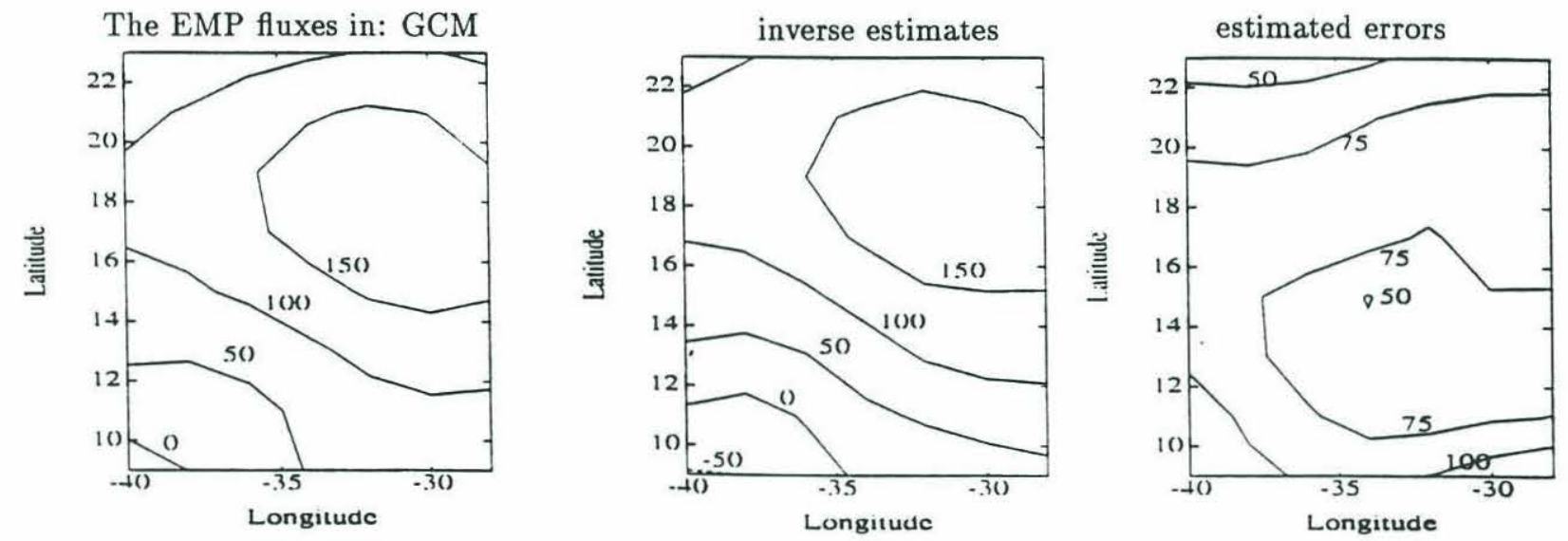

Fig. 3.17. Air-sea heat (upper panel, $W / \mathrm{m}^{2}$ ) and fresh water (lower panel, $\mathrm{cm} / y r$ ) fluxes in: (a) the GCM (the 1st column); (b) the inverse model estimates (the 2nd column) and their expected errors (the 3rd column) from the Upper-Layer Model with frictional surface layer. 


\subsubsection{Deep-Layer Model (DLM)}

As mentioned before, the data "noises" (the neglected temporal varying terms) in the steady inverse model conservation equations for water properties are quite large. Their magnitudes are of, or even larger than, those of the diffusion terms in the deep levels (Fig. 3.5). In this section we will see how these noises will affect the inverse model solutions.

In this experiment, six deeper levels are chosen with the cells centered at the depths of $300,500,800,1250,1750$ and 2500 meters. In the steady inverse model conservation equations for heat and salt, the magnitudes of the data "noise" are smaller than those of the diffusion terms (and thus advection terms) on the two top-most levels (300 and 500 meters) and larger than those of the diffusion terms on other deeper levels. The equation scaling factors are chosen, as in section 3.3.2, as the horizontally averaged norm of the data "noises".

The inverse model solutions for the horizontal circulations are again generally consistent with the numerical GCM data in both patterns and values within the estimated errors. The estimated vertical velocity fields are similar to the horizontally smoothed vertical velocity fields of the "raw" numerical GCM data, and the values are generally consistent within the estimated error bars. The estimated values for the horizontal and vertical diffusion coefficients are $A=(0.98 \pm 0.01) \times 10^{7} \mathrm{~cm}^{2} / \mathrm{s}$ and $K=0.86 \pm 0.02 \mathrm{~cm}^{2} / \mathrm{s}$. Deviation of the estimated value for vertical diffusivity $K$ from its numerical GCM value becomes larger in this lower-layer model (than in the upper-layer model in section 3.3.2). In obtaining the inverse model solutions, the requirement of the minimization of the equation residual norm force the large, neglected temporal terms (the data "noise") into the "physical" terms 
(the advection and diffusion terms). The terms which are most easily adjustable are the diffusion ones, as the diffusive parameters $A$ and $K$ only appear in the heat and salt conservation equations, while the parameters for horizontal circulation and vertical velocity are further constrained by other equations (the dynamic and continuity equations). In the upper layers where advection terms dominate diffusion terms, the equation residuals are more sensitive to the change of velocities than to the change of the diffusivities. Without alternating the residuals greatly, adjusting the diffusive parameters $A$ and $K$ is thus easier than adjusting the parameters for the horizontal circulation and vertical velocity.

To further see the effects of the data "noises" on the inverse model solutions, an experiment is carried out allowing the horizontal diffusion coefficient $A$ to vary with depth but still be constant horizontally (a total of 6 unknown constants for the 6 vertical levels). Although the number of unknowns is increased, the singular value analysis and the analysis of the Levenberg-Marquardt stabilization diagram show that the equation system is still overdetermined and of full rank. The inverse model solutions for the horizontal and vertical velocities are quite robust-they are not significantly different from the previous case (where the horizontal diffusion coefficient $A$ was taken as one unknown constant for all levels). The estimated value for the vertical diffusion coefficient is that $K=0.82 \pm 0.02 \mathrm{~cm}^{2} / \mathrm{s}$, and the those for the horizontal ones are $A=(1.03 \pm 0.03,1.01 \pm 0.03,0.92 \pm 0.03,0.94 \pm$ $0.04,0.84 \pm 0.04,0.64 \pm 0.04) \times 10^{7} \mathrm{~cm}^{2} / \mathrm{s}$ on the six levels respectively. On levels 1 and 2, the inverse model solutions for $A$ are statistically consistent with the GCM data value, while on the deep levels, deviations of the estimates from the GCM data value become significant. This is accounted by the fact that the inverse model solutions try to "parameterize" the large data "noises" as the diffusion (and other) 
terms at the requirement of minimizing the equation residual norm (In this case, the horizontal diffusion coefficient $A$ has the most flexibility, and thus it is the most easily adjustable parameter).

\subsubsection{A Full-Layer Model-Can We Make Consistent Esti- mates?}

In the previous sections we have investigated the inverse model in different parts of the water column with different emphases. In the first place (section 3.3.2) we have applied the inverse model in the upper water column where the assumptions in formulating the inverse model have the most accuracy with the objective to see how well the inverse model works. In the next section, effects of the Ekman pumping velocity constraint on the solutions were examined. After that, a model for the surface frictional layer to estimate the air-sea heat and fresh water fluxes was tested. In the last section (section 3:3.5), effects of the data "noise" on the inverse solutions were examined.

In this section, after all the lessons learned from the previous experiments, we will run the full-layer inverse model in the whole water column.(FLM). First, based on the previous experiments, a standard run will be made and the results will be analyzed in detail in the domain of the numerical GCM ocean. It will be noticed that although the inverse model results are very similar and close to the numerical GCM data (their "true" values), the small discrepancies are significantly above their estimated error bars for some parameters. After that, we will explore the possibilities of making consistent estimates (with their "true" values), using inverse techniques and known physics of the GCM ocean. 


\section{Standard Run}

In this run the model domain covers the whole water column (from the ocean surface to the bottom at $4000 \mathrm{~m}$, with a total of 10 vertical layers). In the surface layer, the model used is the same one as used in section 3.3.4. The rigid-lid assumption $(w=0)$ is used at the sea surface. At the ocean bottom (of $4000 \mathrm{~m}$ ), no normal flow $(w=0)$ and no normal heat and salt fluxes are specified.

The parameters to be estimated are the horizontal and vertical diffusion coefficients $A$ and $K$, the streamfunctions $\psi$ for the horizontal circulations, the vertical velocities $w$, and the air-sea heat and fresh water fluxes. To be consistent with the numerical GCM, the diffusion coefficients $A$ and $K$ are treated as two unknown constants throughout the ocean, while the streamfunction $\psi$ and the vertical velocity $w$ are kept as point-wise unknowns. The equations used are the dynamic equation, continuity equation, and conservation equations for heat and salt. The scaling factors for the water property conservation equations are chosen as the horizontally averaged data "noise" (the temporal variation terms in the numerical GCM data), thus these factors are depth-dependent. As there are more unknowns to be estimated in the surface layer than in the deep layers, and also as the equations in this layer have less accuracy, the equations in the surface layer are down-weighted by a factor of 0.01 .

Writing the constraint equations in the finite difference form on the $9 \times 10 \times 10$ grid points results in 2319 equations for 1328 unknowns. A full examination of the singular values of the equation system combined with the Levenberg-Marquardt stabilization analysis shows that this equation system is overdetermined and of full rank. Therefore all the eigenvalues and corresponding eigenvectors are used in 
obtaining the inverse model solutions for the parameters. Obtained in this way, no restrictions are applied on the size of solution (or parameter) norm. Yet the inverse model results are that all the parameter solutions are of reasonable and expected magnitudes (the unknowns are scaled/normalized to be of order unity). This is another indication of the non-singularity of the equation system.

The inverse model estimated circulations are shown in Fig. 3.18 together with their uncertainties. Comparisons of Fig. 3.18 with Fig. 3.6 show that the inverse model estimated circulations are very similar to those in the numerical GCM ocean on all the levels. On levels 4, 5, 6 and 7 at the depths of 300, 500, 800 and 1250 meters, the estimated circulations are not only in the same patterns of the GCM ones, the velocities are also consistent within the estimated error bars. As the errors on those levels are very small, the consistency means that the estimates are about the same as their "true" values. On the top level at $25 \mathrm{~m}$, the inverse estimates of the circulations are also statistically consistent with the numerical GCM circulations, and the errors in this surface layer are much larger than those in the deep layers. Compared to the numerical GCM circulations, the estimates are rotated anti-clockwisely. The rotations are most obvious in the southern region of the inverse model domain, and they are very small in the northern part. On all the other levels (at the depths of $75,150,1750,2500$, and 3000 meters), although the estimates of the horizontal circulations are very close to their numerical GCM ones in both patterns and values, the differences are small but significant within their even smaller estimated errors. Those significant differences may be accounted for by the different physics in the inverse model and those in the numerical GCM. For example, in the numerical GCM fields, the thermal wind relations can have relative errors as large as $20 \%$ in zonal velocity and $10 \%$ in meridional velocity. 
The full-layer model estimated vertical velocities and their errors are shown in Fig. 3.19a and Fig. 3.19b respectively. On the top three levels, the inverse solutions are very similar to the GCM data (Fig. 3.7a) in both patterns and magnitudes. However, moving deeper and deeper (at the depth of 400,600, 1000, 1500, 2000 and 3000 meters), the differences between the estimates and the GCM data become more and more apparent. The numerical GCM vertical velocities (Fig. 3.7a) are very scattered in space on those levels, while the inverse model solutions are smooth and unable to resolve those small scale structures. In fact, the inverse model solutions are more similar to the spatially smoothed fields of the numerical GCM vertical velocities (Fig. 3.7b). It can be seen that the vertical velocities in Fig. 3.19a and Fig. 3.7b have similar distribution patterns and magnitudes. In this case the inverse model results are not sensitive to the random data "noise".

The estimated heat and fresh water fluxes at air-sea interface are about the same as those estimates in section 3.3.4 (Fig. 3.17), and they are statistically consistent with the values used in the numerical GCM.

The estimated values for the horizontal and vertical diffusive coefficients are $A=(1.09 \pm 0.02) \times 10^{7} \mathrm{~cm}^{2} / \mathrm{s}$ and $K=1.03 \pm 0.02 \mathrm{~cm}^{2} / \mathrm{s}$. In terms of closeness to their "true" values, these estimates are better than those in the deeplayer model results (section 3.3.5) but worse than those in the upper-layer model results (section 3.3.2). Although these values are very close to the numerical GCM values (the relative errors are $9 \%$ and $3 \%$ respectively), their small differences are significant within the estimated error bars. These significant biases are accounted by the discrepancies between the inverse model and the GCM, as analyzed before (section 3.3.2). 
Obtaining the solutions and the solution errors is not the complete story. We should also check whether our estimations are consistent with our a priori assumptions used in obtaining the solutions. In choosing the equation scaling factors and in computing the expected solution errors, we have used the assumption that the data noises in the equations are "white" (i.e. uncorrelated with zero mean). The post-solution residuals in the equations in this case are shown in Fig. 3.20a. It can be seen that although not totally white, these residuals are more or less randomly distributed, and the mean of them are much smaller (of two orders) than the magnitude of the residuals (and thus can be loosely regarded as zero mean). The auto-correlations of these residuals are shown in Fig. 3.20b together with their $95 \%$ confidence error bounds (c.f. section 4.2 for detail). This figure shows that the correlation coefficients decrease from unity at zero lag to below 0.1 very rapidly, and the values are generally insignificant beyond zero lag, which indicates that the post-solution noises in the equations are independent. 

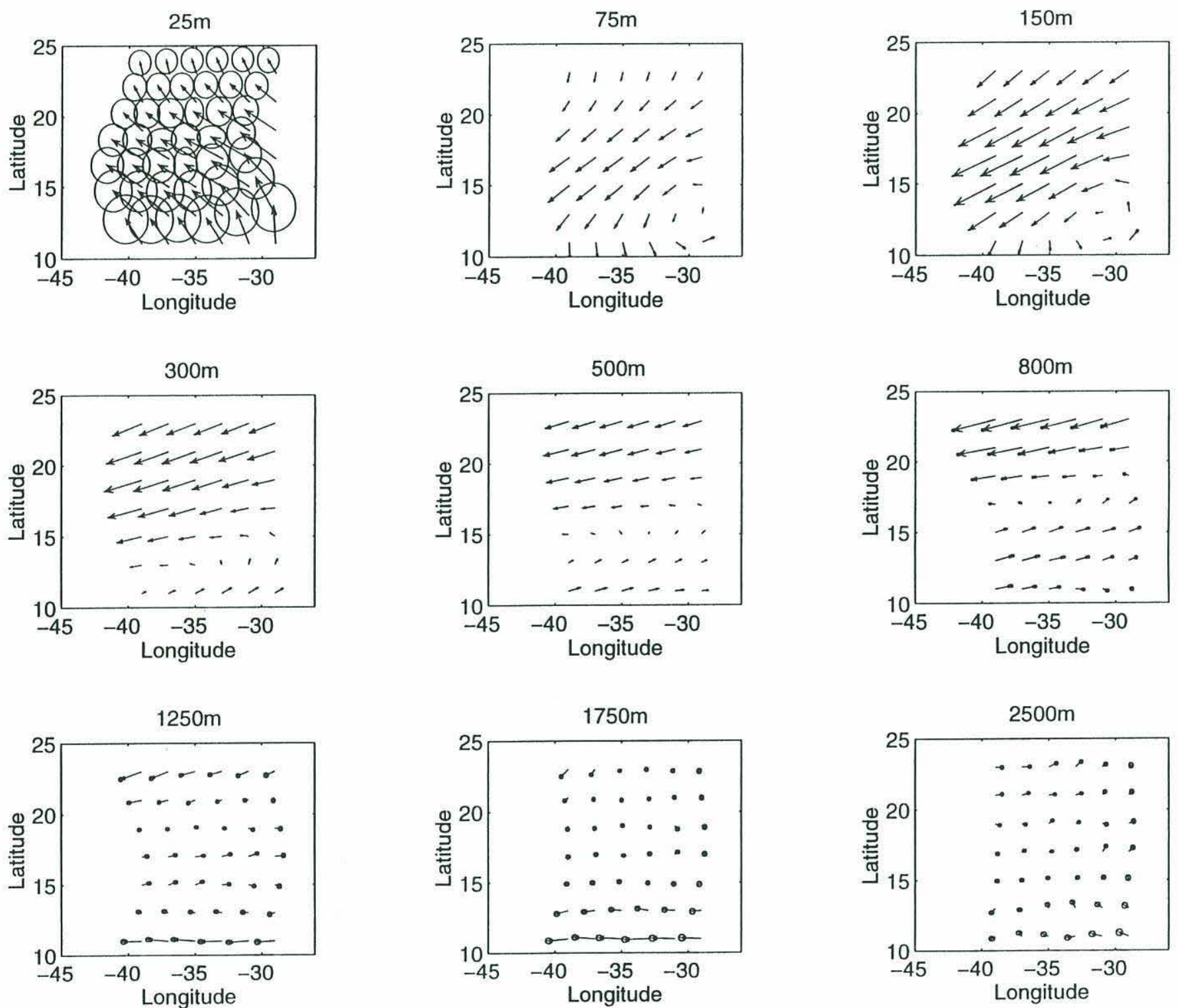

Fig. 3.18. Estimated horizontal circulations with their error ellipses from the standard run.

Vector scales are the same as in Fig. 3.6. 

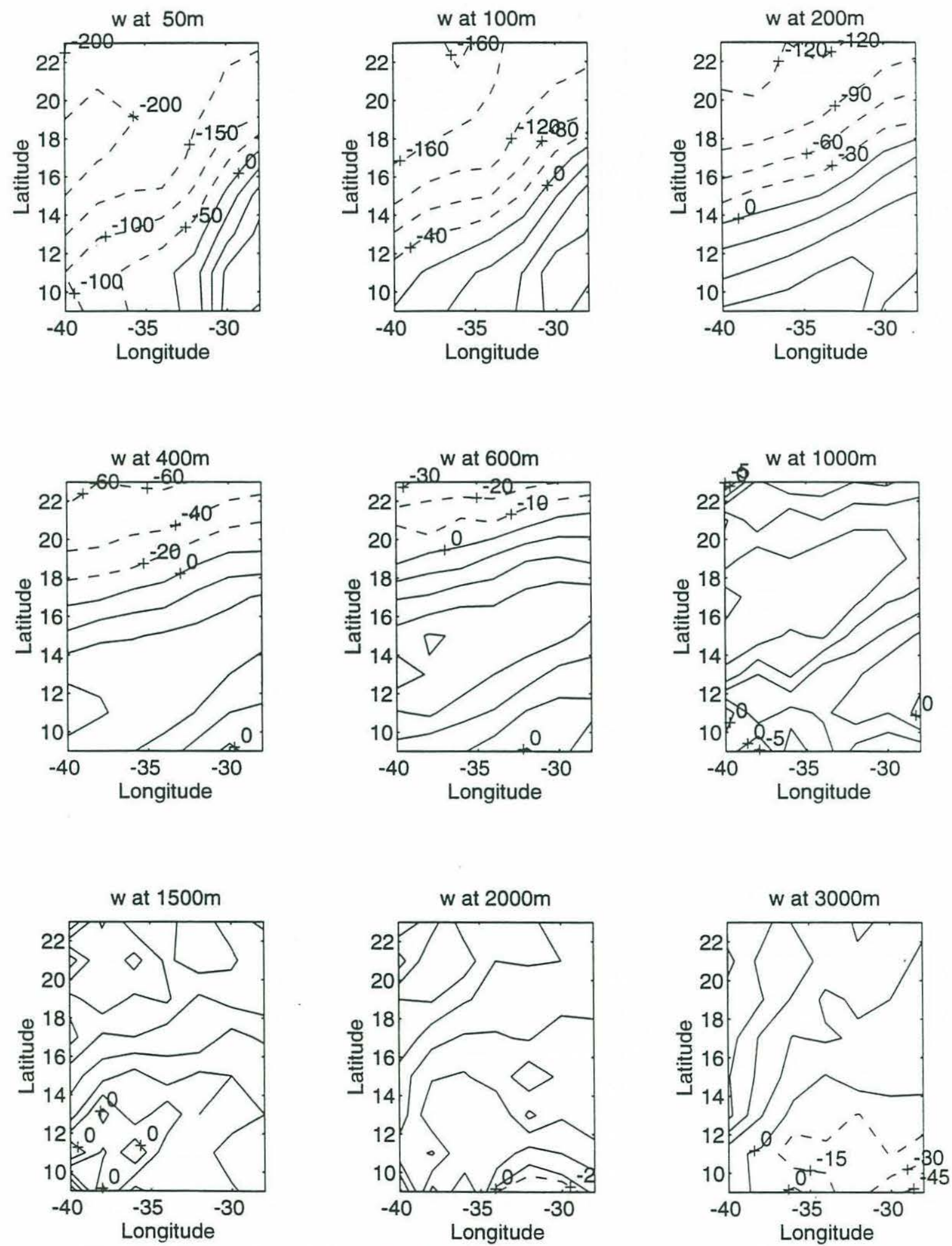

Fig. 3.19. Estimated vertical velocities (a) and their expected errors (b) from the standard run. Units are $\left(10^{-6} \mathrm{~cm} / \mathrm{s}\right)$. 

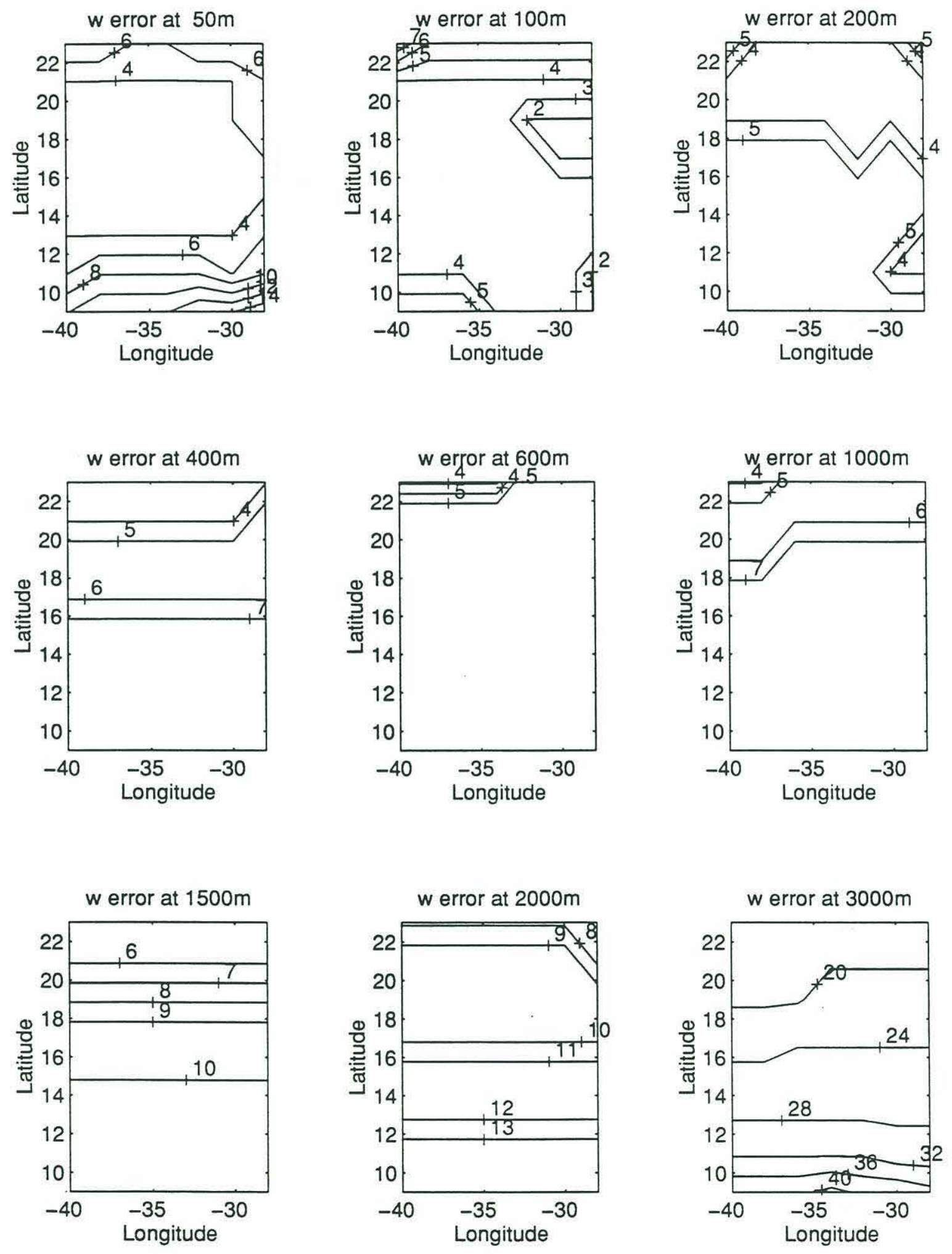

Fig. 3.19. (b) 

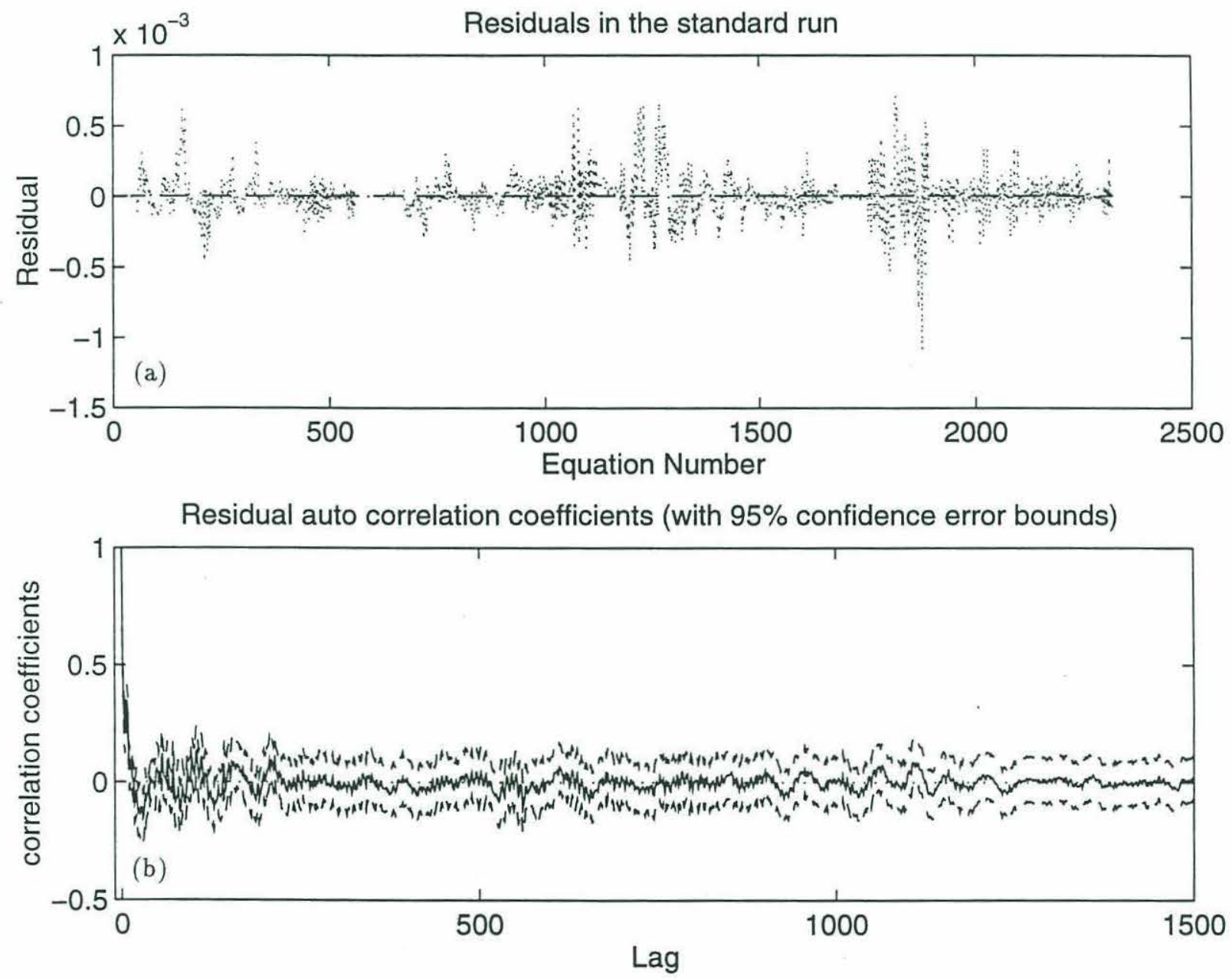

Fig. 3.20. Post-solution equation residuals (a) and their auto correlations (b) of the standard run. 


\section{Effects of the Temporal Variation Terms on Inverse Solutions (IH)}

From all the experiments in previous sections, we can see that although the temporal variation terms are large at great depth and are neglected in the inverse model, we can still obtain parameter estimates which are very close to their numerical GCM data values (for example, the relative errors in the horizontal and vertical diffusion coefficients $A$ and $K$ are only $9 \%$ and $3 \%$ respectively in the case of the standard run). However, we also noticed that although the differences between the inverse model solutions and their "true" values are very small, these differences are yet significantly above the estimated errors for some parameters at certain locations. We have not been able to find completely consistent solutions for all parameters due to the discrepancies between the inverse model and the GCM. In one aspect, the GCM ocean is not in complete steady state, while the inverse model is a steady one. In our special data set of the GCM ocean, the temporal variation terms in the conservation equations for heat and salt are known. They are very scattered in space, but not in total random distribution (non-white). This information can be utilized to examine how the inverse model solutions will be affected by the non-white "noises".

In the experiment in this section, the temporal variation terms are treated as knowns (the inhomogeneous terms) on the right hand side of the conservation equations for heat and salt (we label this experiment as $\mathrm{IH}$ ). With this information utilized, the conservations are satisfied exactly by the GCM data, and thus the depth-dependent equation scaling factors based on the "noise" (temporal variation) levels (used in previous sections) should be removed. The equation scaling factors in this case are chosen as in Zhang and Hogg (1992), namely the horizontally averaged tracer anomalies. Also, as the temporal variation terms are included in the inverse 
model, the accuracy in the tracer conservation equations is increased, and thus higher weights should be given to these equations. In this run, we down-weighted the dynamic equations from a factor of 0.25 to a factor of 0.09 . This is chosen on experimental bases so that the post-solution residuals in the dynamic equations and the conservation equations are in the same magnitude.

With these changes, we run the full-layer model again. As expected, the equation system is overdetermined and of full rank. The estimated circulations are shown in Fig. 3.21 together with their error ellipses. Comparisons of Fig. 3.21 with the estimated circulations in the standard run (Fig. 3.18) as well as those in the GCM ocean (Fig. 3.6) show that the solutions themselves for the horizontal velocities are relatively robust (not sensitive to the "noises" or the temporal variation terms). However, compared to the standard run, the estimated errors in this run are increased significantly, especially on the deep levels. With the increase of these errors, the estimated horizontal circulations are completely consistent with the GCM ones. The solutions for horizontal velocity have not been improved (toward their "true" values) compared to the standard run, since the formulation and accuracy of the dynamic equations have not been improved.

What are improved in this case are the conservation equations for heat and salt, and therefore we would expect that the inverse model solutions for the tracer diffusion coefficients should be improved toward their true values (as these parameters only appear in the tracer conservation equations). As a matter of fact, the inverse model estimates for these parameters are $A=(1.03 \pm 0.05) \times 10^{7} \mathrm{~cm}^{2} / \mathrm{s}$ and $K=0.99 \pm 0.02 \mathrm{~cm}^{2} / \mathrm{s}$. Not only these estimations are closer to their numerical GCM data (the relative errors are reduced to $3 \%$ and $1 \%$ ), but also they are consistent within the estimated errors. 

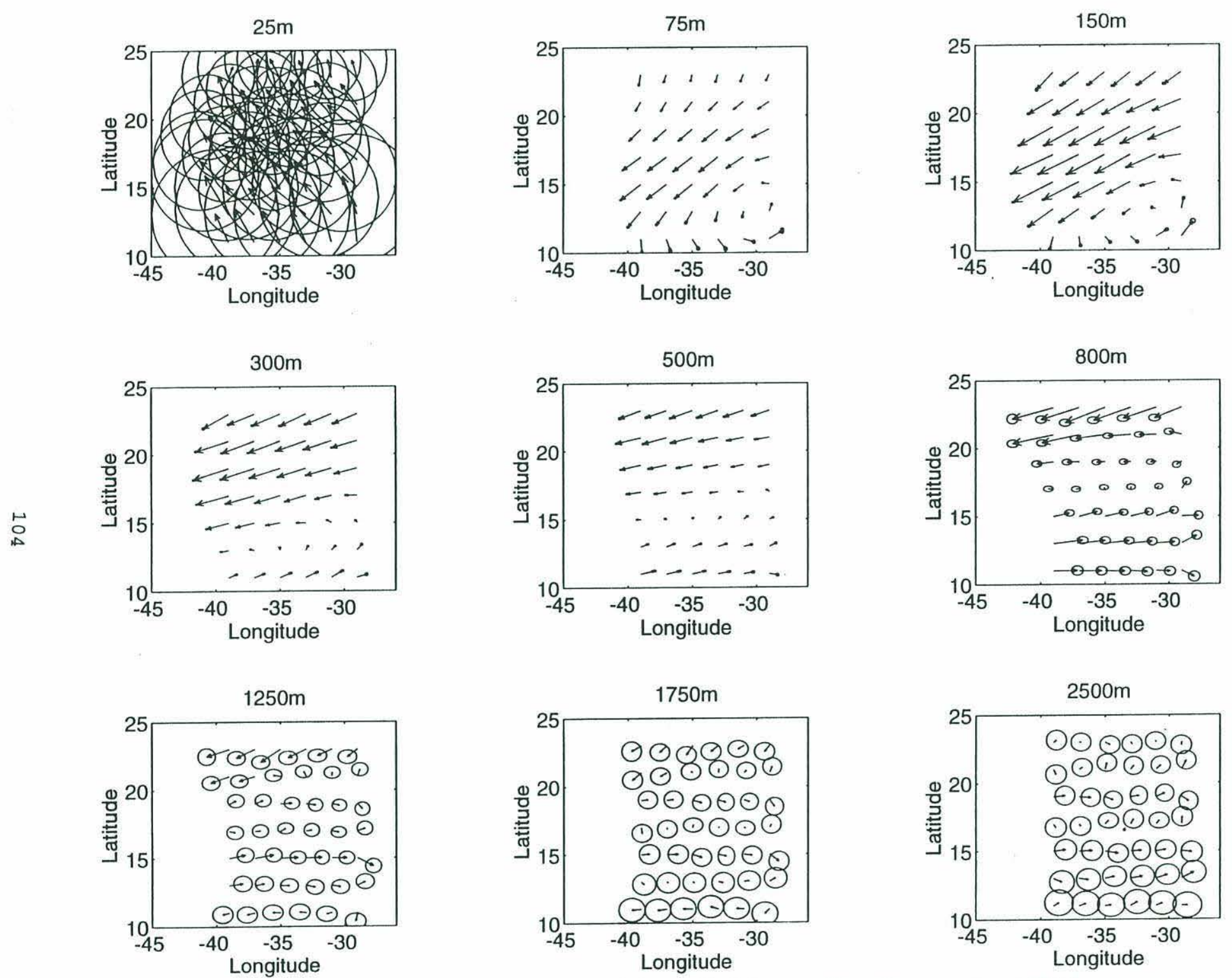

Fig. 3.21. Estimated horizontal circulations with their error ellipses from experiment IH (the temporal variations as known inhomogeneous RHS). Vector scales are the same as in Fig. 3.6. 

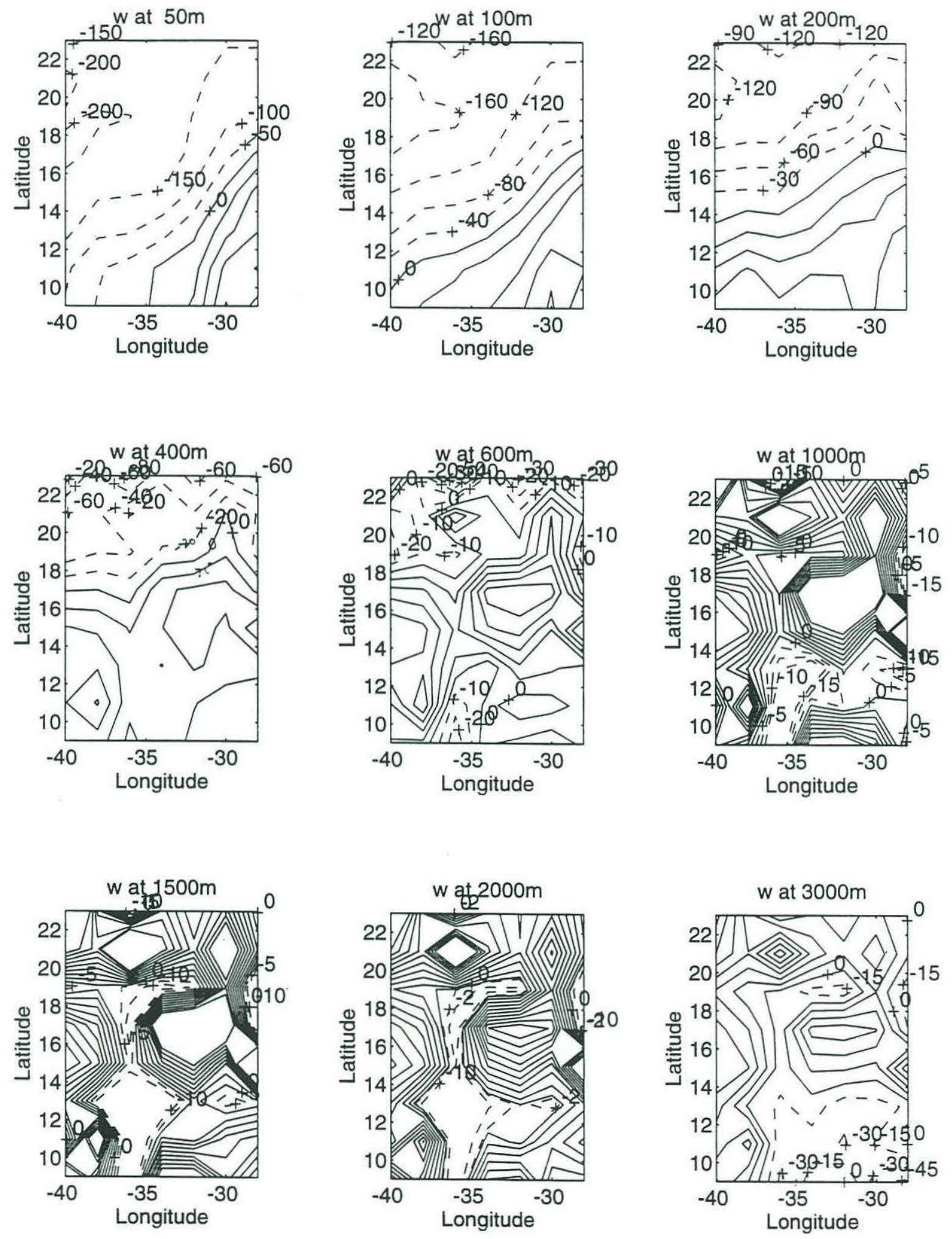

Fig. 3.22. Same as in Fig. 3.21 but for vertical velocities (a) and their estimated errors (unit: $\left.10^{-6} \mathrm{~cm} / \mathrm{s}\right)$. 

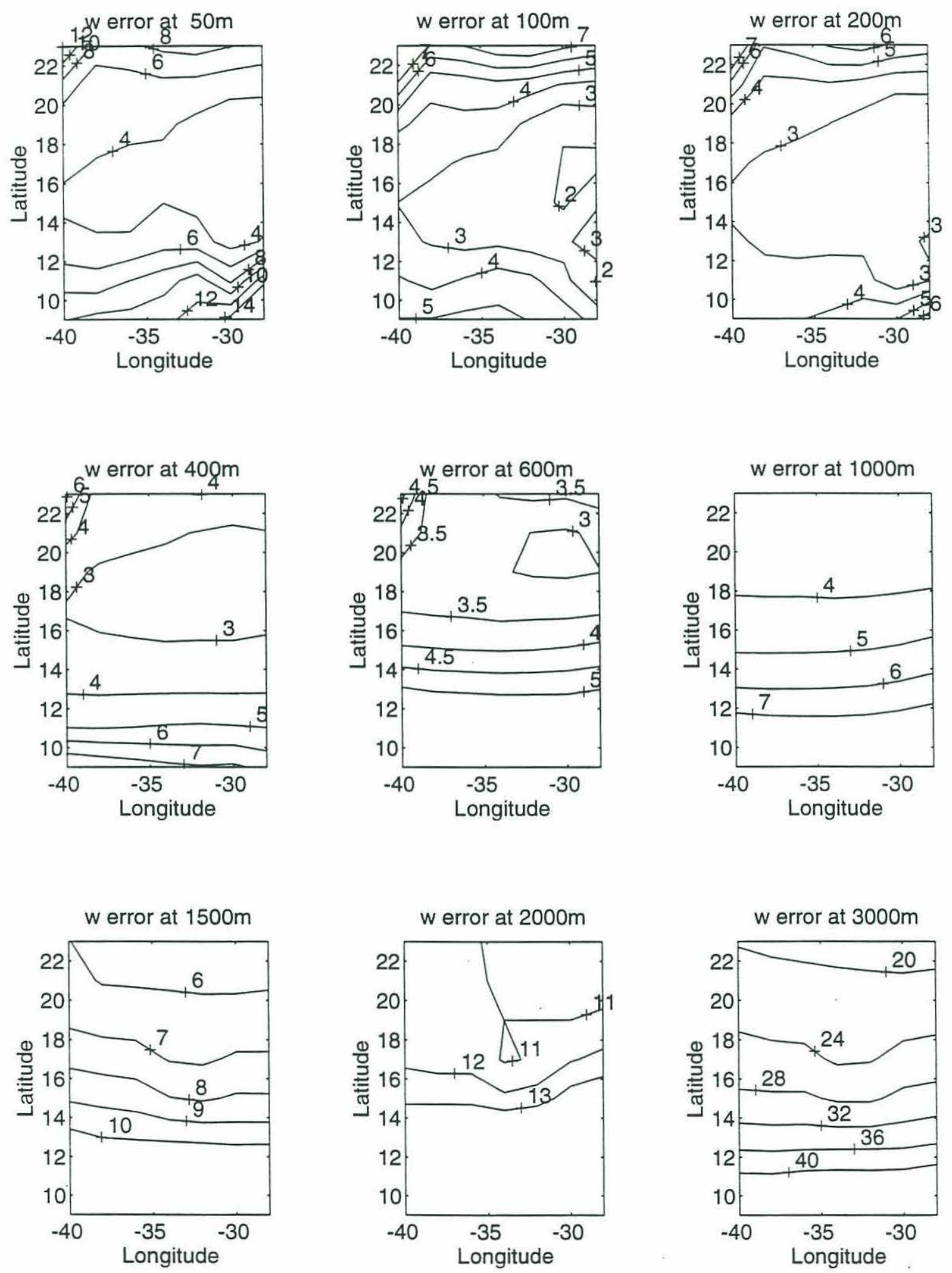

Fig. 3.22 (b) 
The distributions of the estimated heat and fresh water fluxes at the sea surface are not as smooth as those from the standard run. But the large scale patterns and magnitudes are still very similar. Numerically, they are also close to the estimations from the standard run and the values of the numerical GCM parameters. In fact, they are statistically consistent with each other.

Compared to the previous experiments, the most obvious and interesting feature of this run is in the estimates for the vertical velocities. In all the experiments in previous sections, time-dependent terms are neglected (except their roles in the equation scaling). We had been able to get estimations for the vertical velocities which are similar to the spatially smoothed GCM fields, but we had not been able to have the inverse model solutions to resolve the small scale structures. With the specification of the temporal variation terms, the inverse model estimates for the vertical velocity (Fig. 3.22) are improved toward their "true" values tremendously, especially on the deep levels. Comparisons of Fig. 3.22 with Fig. 3.7a show that these estimates are about the same as the GCM vertical velocities in small scale structures. Numerically, they are also consistent with the estimated error bars. This example shows how a correct model could not fail to produce the right answers.

The sensitivity of the inverse model solutions for the vertical velocity to the temporal variation terms can be explained by the fact that, in the heat and salt conservation equations in the numerical GCM ocean, the temporal variation terms have the same structures as those of the vertical advection terms $(\partial(w T) / \partial z)$ on the deep levels (for example, at the levels deeper than $800 \mathrm{~m}$, see Fig. 3.5 and Fig. 3.7a). As a result, neglecting the temporal variation terms in the inverse model, or equivalently, taking them as homogenous values of zero (acting as roles of horizontal smoothing in the whole area), will have the most impacts on advection 
terms $\partial(w T) / \partial z$. As the tracer $T$ fields are specified and invariable, the only way to change the structures of $\partial(w T) / \partial z$ is by altering the solutions for the vertical velocity $w$. Because the horizontal and vertical diffusion coefficients are taken as two constants through the whole water column and in the whole inverse model domain, and because the solutions for the horizontal circulations are further constrained by the dynamic equations, it is much more difficult to alter their solutions to pick up the change of the small scale structures of the temporal variation terms.

Although the magnitudes of the temporal variation terms in the heat and salt conservation equations are quite large compared to the diffusion terms (especially at great depths), the horizontally scattered structures of them do simulate the distributions of random noises to some extent. Therefore the results of the above experiments do have some relevance to the inverse studies of the ocean circulations and diffusion processes in the real ocean, where the data noises are usually unknown. In the above experiments, we can see that, neglecting the randomly distributed data "noises" in the inverse model, we can get spatially smoothed estimates for the parameters. It should also be pointed out that the solutions for the horizontal velocities are more robust than those for the other parameters (diffusion coefficients and vertical velocities).

The conclusions above are drawn from the comparisons of the results of the above experiment $(\mathrm{IH})$ with the results of the standard run. There are two differences between the setups of $\mathrm{IH}$ and the standard run. One is that the scaling factors are different: the depth-dependent row scaling factors of the "noises" (the temporal variation) levels were used in the standard run, while they were removed from $\mathrm{IH}$. The other aspect is the specification of the temporal variations in the heat and salt conservation equations in IH, while they were "neglected" in the standard 
run (except their roles in the equation scalings). In order to justify our conclusions above, we need to isolate the individual effects of the above two aspects on the changes of the inverse model solutions. To do so, we run a mixed experiment (labeled with MIX) in which the depth-dependent row scaling factors are not used (as in $\mathrm{IH}$ ) while the temporal variations are also neglected in the conservation equations (as in the standard run).

The inverse model solutions for the horizontal circulations in this case (MIX) are closer to those in IH (in fact they are statistically indistinguishable) than to those in the standard run. Among the three sets of solutions for the horizontal velocities, those in the standard run are closest to their "true" values, and they possess the smallest uncertainties (Note that in the three experiments, the depth-dependent row scaling factors were only used in the standard run). For the vertical velocities, the inverse model estimates in MIX are about the same as those in the standard run, but the estimated errors are larger. The distributions of these solutions are similar to the horizontally smoothed fields of the numerical GCM vertical velocities (Fig. 3.7b), and thus are different, in small scale structures, from those of the "raw" GCM data (Fig. 3.7a) and those in IH. The inverse model estimated diffusion coefficients in this case (MIX) are $A=(1.05 \pm 0.03) \times 10^{7} \mathrm{~cm}^{2} / \mathrm{s}$ and $K=0.99 \pm 0.02 \mathrm{~cm}^{2} / \mathrm{s}$. In terms of closeness to their "true" values, these solutions are better than those in the standard run, but not as good as those in IH. The inter-comparisons of the above three experiments show that the solutions for the vertical velocities are most effectively affected by the inclusion of the temporal variation terms in the conservation equations for heat and salt, while the estimates for the horizontal circulations are most sensitive to the changes in the row scaling factors of the equations. All these experiments result in reasonably good estimates in large scale, 
which indicates that in this study, the scaling factors and data "noises" are not crucially important for the solutions.

\subsection{EXPERIMENTS ON THE PARAMETERIZATION}

\subsubsection{Introduction}

In all the experiments in the previous sections, the horizontal and vertical diffusion coefficients were treated as two constant unknowns throughout the whole water column and in the whole inverse model domain (the only exception is the experiment in the deep-layer model where vertical variation of the horizontal diffusion coefficient $A$ was allowed). This treatment was adopted in order to keep the inverse model scheme as close to that of the numerical GCM as possible. In the real ocean, the turbulent diffusion coefficients used to parameterize the water property fluxes produced by subgrid/meso scale turbulent processes are generally functions of the turbulent kinetic energy of the motions, and thus functions of space in the horizontal as well as in the vertical. In some cases, the diffusion coefficients are not only inhomogeneous, but also not isotropic (e.g., Figueroa and Olson, 1989; Spall et al, 1993). In these cases the above constant-diffusion-coefficient approaches may be too simplified.

A most straight forward approach to compute these spatially varying diffusion coefficients from the inverse model is to allow them to have as many degrees of freedom as possible. We could treat these parameters as point-wise unknowns (like the parameters for the horizontal and vertical velocities), and even let the horizontal diffusion coefficients to be different in the zonal and meridional directions. With 
these formulations, the inverse model usually produces an equation system which composes more unknowns than equations, and thus the equation system becomes under-determined.

For the under-determined system, the inverse model solutions are usually the results of minimizing the solution norm. In the inverse models formulated for the "reference" velocities (as unknowns to be estimated), the reference levels are usually chosen where the circulations are the "weakest", and the kinetic energy is minimized. In the inverse model formulated in this work, the parameters for the horizontal circulations on all the vertical levels are solved simultaneously, together with the estimations of the parameters for the vertical velocity and diffusion coefficients. If the degrees of freedom (in space) of the diffusive parameters are so large that the equation system is under-determined, what should be minimized in obtaining the solutions is the norm of the deviations of the estimated parameter values from their true values. In the real ocean, these true values are usually unknown (otherwise we do not need to do the inversion at all), and the specifications of them are rather subjective and difficult.

On the other hand, if we can formulate an over-determined system with full rank, the inverse model solutions are then obtained by minimizing the residual norm of the equations. Minimization of the solution norm is not required, and thus the solutions are not sensitive to the a prior knowledge of the true values of the parameters. In this sense, the solutions from an over-determined equation system are more objective. Note that even for the over-determined system with full rank, there are still some subjective factors in obtaining the inverse model solutions (such as the row scaling factors of the equations) 
In order to represent the spatial variations of the diffusive parameters, while at the same time to pose an over-determined equation system, the diffusion parameters are usually parameterized as polynomial functions in space (e.g., Olbers et al, 1985; Hogg, 1987; Tziperman, 1988). In this section, using the available information in the numerical GCM ocean, the effects of the parameterization of these diffusive parameters on the inverse model results will be studied. The following experiments are based on the configurations of the standard run (i.e., using the same scaling factors and the temporal variation terms being neglected in the equations).

\subsection{2 $A, K, w$ and Air-Sea Fluxes as $3^{r d}$ Order Polynomials- PAR I}

In the first experiment (labeled as PAR I), the parameters for horizontal and vertical diffusion coefficients and vertical velocity $A, K, w$ as well as those for the air-sea heat and fresh water fluxes are all parameterized as the third-order discrete Tchebychev polynomial functions in the horizontal plane, with the coefficients varying with depth and being different for different parameters. The equation system consists of 2319 equations for 1010 unknowns, and it is apparently over-determined. The singular value and the Levenberg-Marquardt stabilization analysis show that the system is of full rank.

The horizontal circulations estimated from this run have the same flow patterns and magnitudes as those in the numerical GCM ocean and those estimated in the standard run on all the vertical levels, and the numerical values are also consistent in mid-depth. However, there are small but yet significant differences in 
velocity values at the surface layer (at $25 \mathrm{~m}$ ) and at greater depths (depths greater than $1250 \mathrm{~m}$, where the horizontal circulations are the weakest).

Parameterized as polynomial functions in space, the solutions for the vertical velocity (Fig. 3.23) have smoother horizontal structures. The patterns and magnitudes are very similar to those estimated in the standard run and those of the 9-point smoothed vertical velocity fields of the GCM ocean. The numerical values are also consistent within the estimated error bars at depths greater than $200 \mathrm{~m}$. At the bottom of the surface layer (at $50 \mathrm{~m}$ ) and the second layer (at 100 $\mathrm{m})$, although the distribution patterns and magnitudes are still very similar, the estimates in this case are significantly different from those in the standard run and those in the horizontally smoothed fields of the numerical GCM vertical velocities, especially at the northwest and southeast corners.

Despite the relatively robust solutions for the horizontal and vertical velocities, the solutions for the horizontal and vertical diffusion coefficients (Fig. 3.24a,b) and the air-sea heat and fresh water fluxes (Fig. 3.25) are not all consistent with the values of the numerical GCM parameters and those in the standard run. Parameterized as polynomial functions, the estimated values for $A$ and $K$ are not constant any more, and they have large scale structures in the horizontal and also vary vertically. In the mid-depth (on the five levels at 150, 300, 500, 800 and 1250 meters), the inverse model estimates are not too far from the GCM parameter values-the area averages of $A$ are of order $1.0 \times 10^{7} \mathrm{~cm}^{2} / \mathrm{s}$ (their "true" value). However, at the other depths, the estimated values for $A$ are obviously different from their "true" values. At the depth of $25 \mathrm{~m}$ (the vertical center of the surface layer), the estimated values for $A$ are larger $\left(\sim 4 \times 10^{7} \mathrm{~cm}^{2} / \mathrm{s}\right)$ than the "true" value, but the differences are insignificant because of the even larger solution errors. On the other 
hand, at the depths of $75 \mathrm{~m}$ and at 1750, 2500 and 3500 meters, the solutions for $A$ are significantly smaller than the "true" value, although they are of the same order. Compared to the poor estimates for $A$, the estimates for the vertical diffusion coefficients $K$ are closer to their "true" value, although the spatial variations are still obvious. The area averages of $K$ are about $1.0 \mathrm{~cm}^{2} / \mathrm{s}$ on all the levels except the deepest one (at $3000 \mathrm{~m}$ ). The solutions for the vertical diffusion coefficients are more robust than those for the horizontal ones.

The biased estimates for the diffusion coefficients, the horizontal and vertical velocities result in biased estimates for the air-sea heat and fresh water fluxes (Fig. 3.25). These estimates are significantly different from those used in the numerical GCM in both pattern and values, although they are of the same order. 
w at $50 \mathrm{~m}$
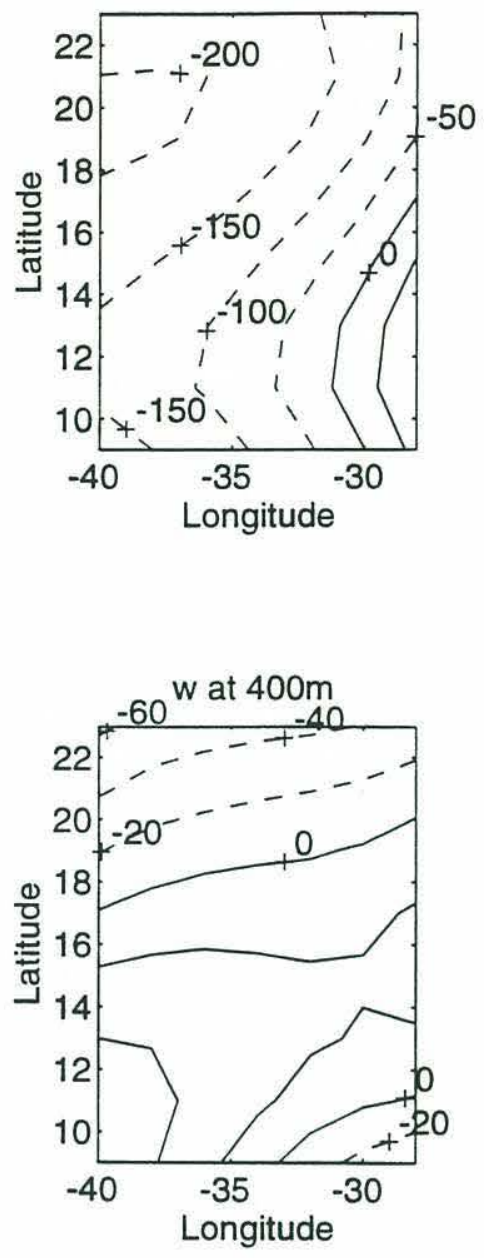

$w$ at $1500 m$

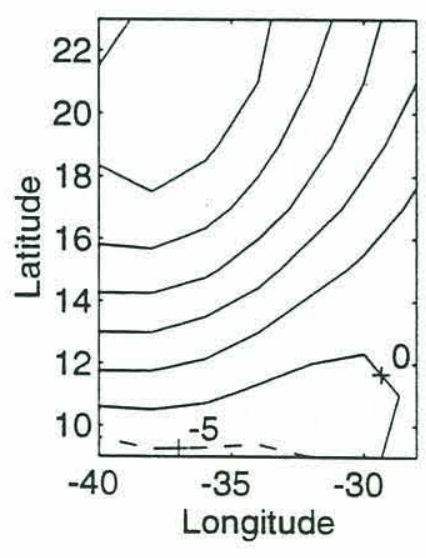

$w$ at $100 m$
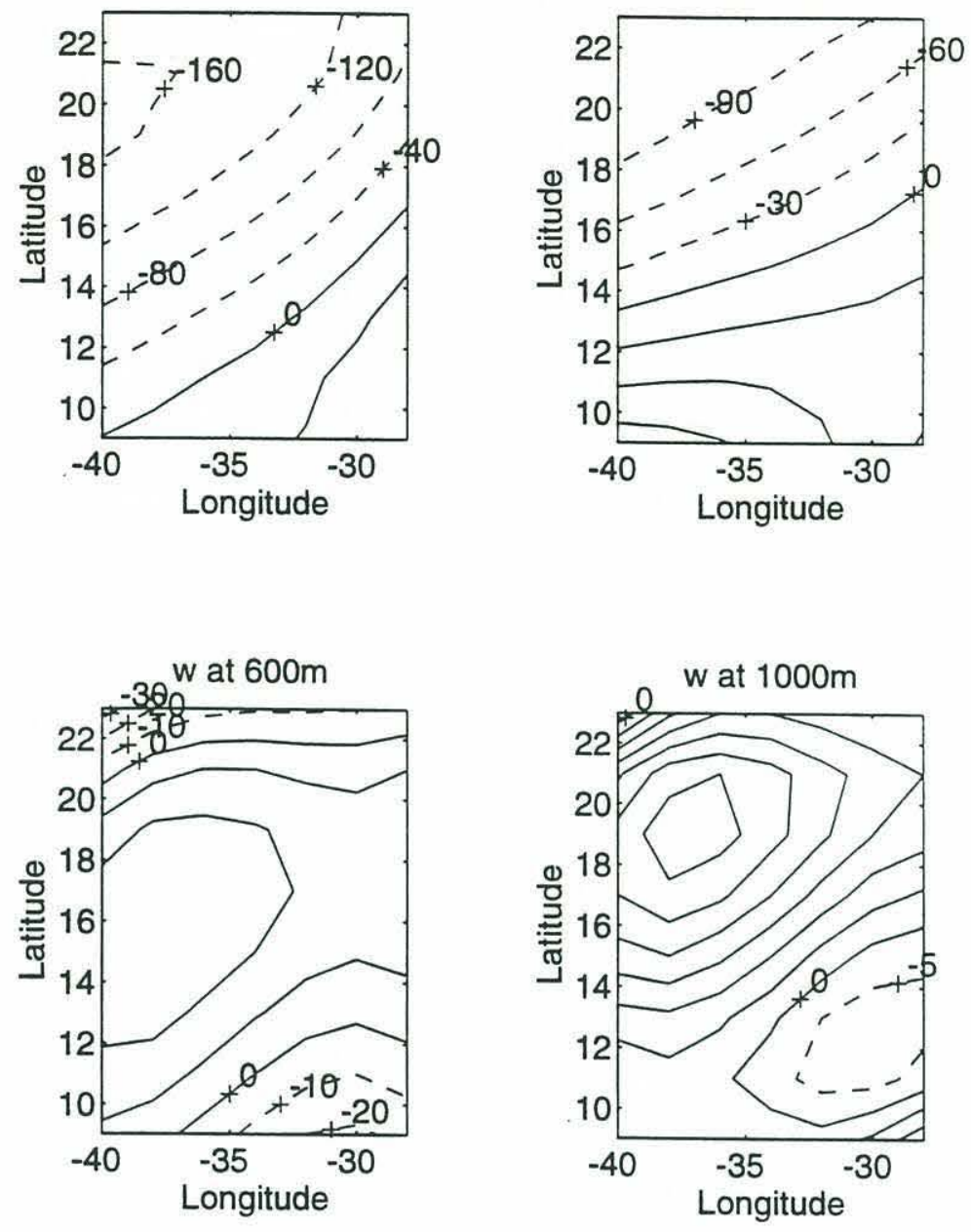
Fig. 3.23. Estimated vertical velocities (a) and their errors (b) from experiment PAR I. (unit:
$10^{-6} \mathrm{~cm} / \mathrm{s}$ ). 

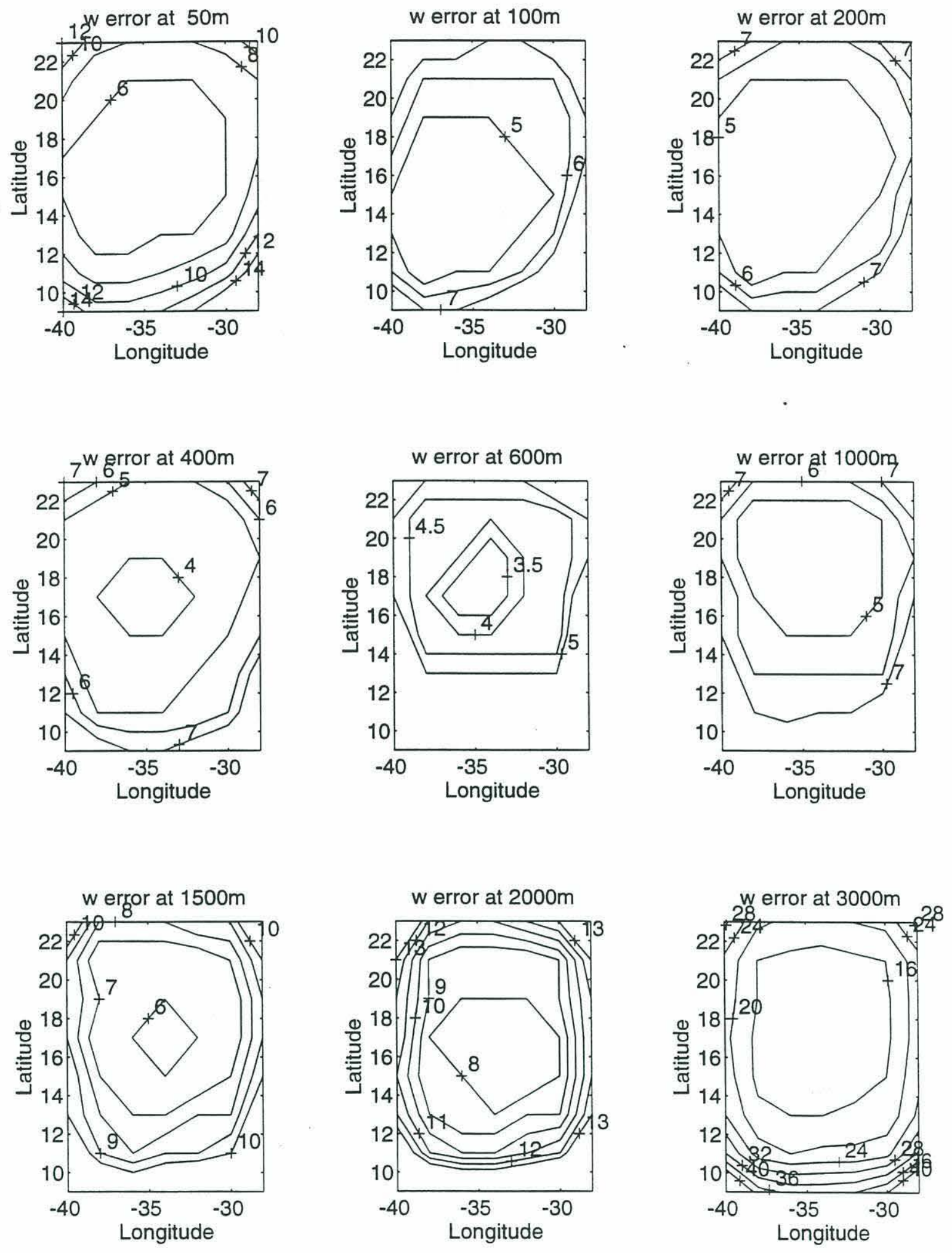

Fig. 3.23. (b) 

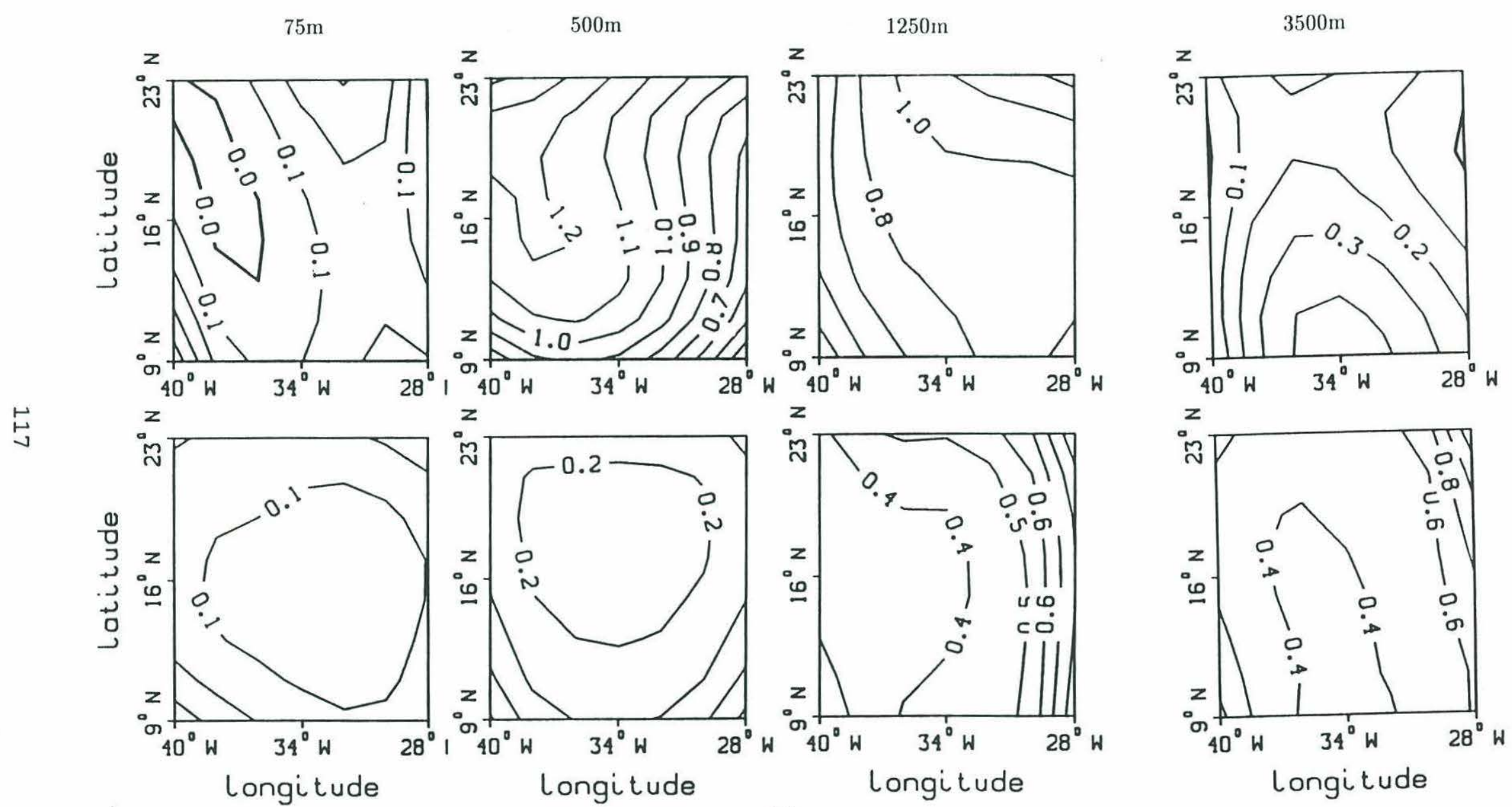

Fig. 3.24. Estimated diffusion coefficients (upper panel) and their errors (lower panel) from experiment PAR I. (a) horizontal diffusivity $\left(10^{7} \mathrm{~cm}^{2} / \mathrm{s}\right)$; (b) vertical diffusivity $\left(\mathrm{cm}^{2} / \mathrm{s}\right)$. 

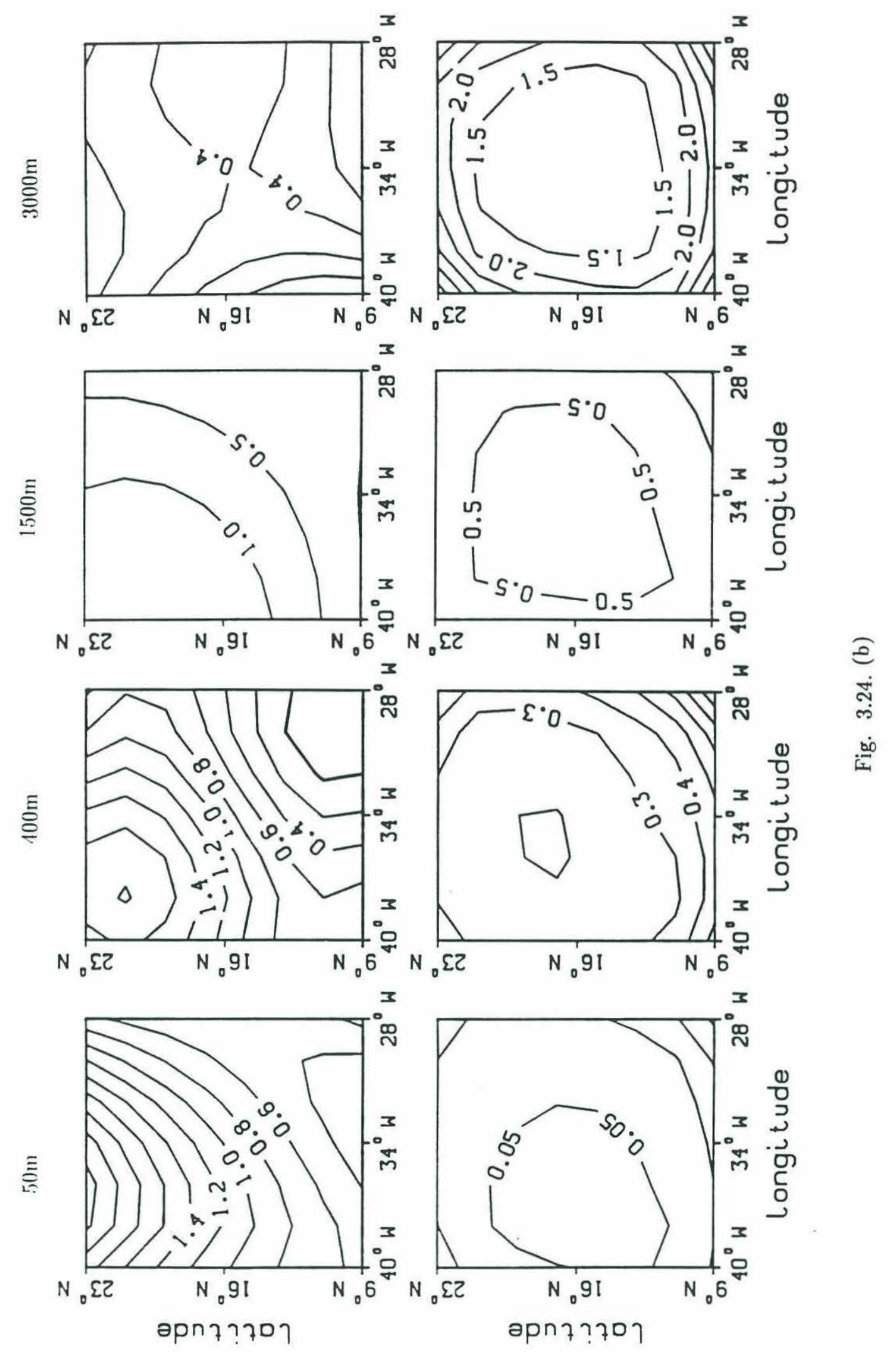

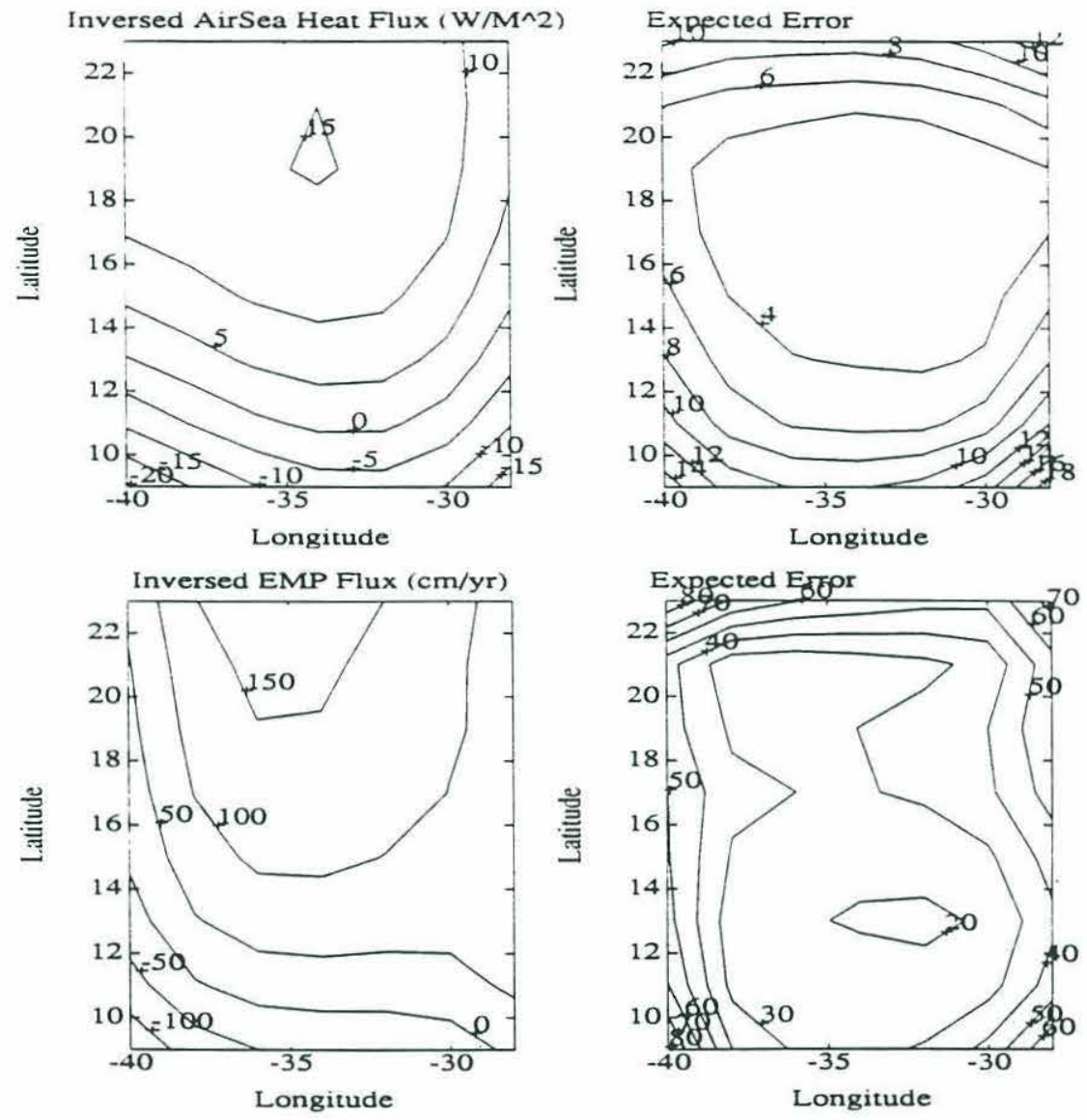

Fig. 3.25. Estimated air-sea heat $\left(W / m^{2}\right)$ and fresh water (EMP, $\left.c m / y r\right)$ fluxes and their errors from experiment PAR I. 


\subsection{3 $A, K$ and Air-Sea Fluxes as $3^{\text {rd }}$ Order Polynomials while $w$ is a Point-wise Unknown-PAR II}

The parameterization scheme used in the previous section (section 3.4.2, PAR I) result in some "unrealistic" features in the inverse model solutions for the air-sea heat and fresh water fluxes as well as for the diffusion coefficients (especially the horizontal ones). If the small values of horizontal diffusivity $A$ on the deepest three levels can be attributed to the large data "noises" in the heat and salt conservation equations, it is difficult to explain the small values of $A$ at the depth of $75 \mathrm{~m}$ and the "biased" estimates for the air-sea heat and fresh water fluxes. These features must be associated with the specific parameterization scheme used in PAR I.

In PAR I, the vertical velocity was treated like a "diffusive" parameter (which was parameterized as polynomial functions). This treatment was borrowed from the concept of the diapycnal velocity, which is caused by diffusion processes. If there is no diffusion at all (both isopycnal and diapycnal), there would be no diapycnal advection. However, in the geopotential $(\mathrm{z})$ coordinate, this is not true any morevertical advections can happen with or without horizontal and vertical diffusion processes.

In the experiment in this section (labeled as PAR II), the parameters for the vertical velocity $w$ are taken as point-wise unknowns as in the standard run, while the parameters for the diffusion coefficients $A, K$ and air-sea heat and fresh water fluxes are parameterized as the third-order discrete Tchebychev polynomial functions as in PAR I. Although there are more unknowns in this case than in PAR I and the standard run (there are 2319 equations for 1424 unknowns), the singular 
value and the Levenberg-Marquardt stabilization analyses show that the system is still overdetermined and of full rank.

The estimated horizontal and vertical velocities in this experiment are improved toward those of the numerical GCM ocean and those in the standard run, and the solution errors are smaller. The estimated diffusion coefficients (Fig. 3.26) still have varying spatial structures, but the magnitudes of deviations from the GCM values are smaller than in PAR I. The area averages of $A$ are around $1.0 \times 10^{7} \mathrm{~cm}^{2} / \mathrm{s}$ on seven (above $1500 \mathrm{~m}$ ) of the ten vertical levels. As in the previous case, the estimates for $A$ are smaller than the GCM values on the deepest three levels (at the depths of 1750, 2500 and 3500 meters), and these biases are caused by the large data "noises" in the steady state conservation equations. But the unexplained small values of $A$ at the depth of $75 \mathrm{~m}$ in PAR I disappeared in the present case, and the solutions are near the numerical GCM values. Furthermore, the estimates for $K$ are closer to their "true" values (of $1 \mathrm{~cm}^{2} / \mathrm{s}$ ) on all the vertical levels.

As the estimates for the horizontal and vertical velocities as well as diffusion coefficients are closer to their "true" values, the inverse model estimated air-sea heat and fresh water fluxes are significantly improved toward their "true" values (Fig. 3.27). Both the estimated patterns and values are much closer to the GCM ones in this case than in PAR I, although they are still not as good as in the standard run. 

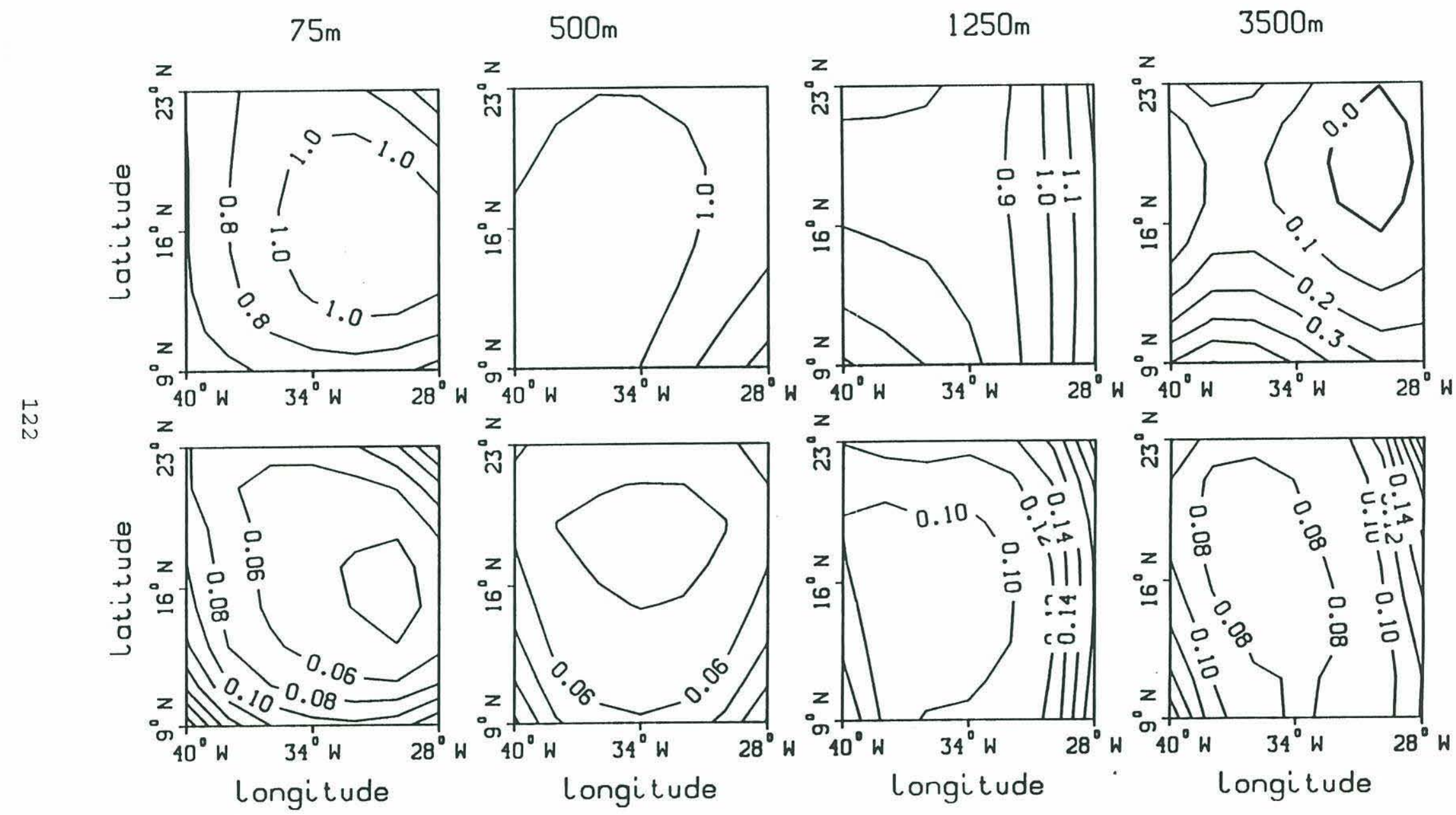

(a) horizontal diffusivity

Fig. 3.26. Same as Fig. 3.24 but from experiment PAR II. 

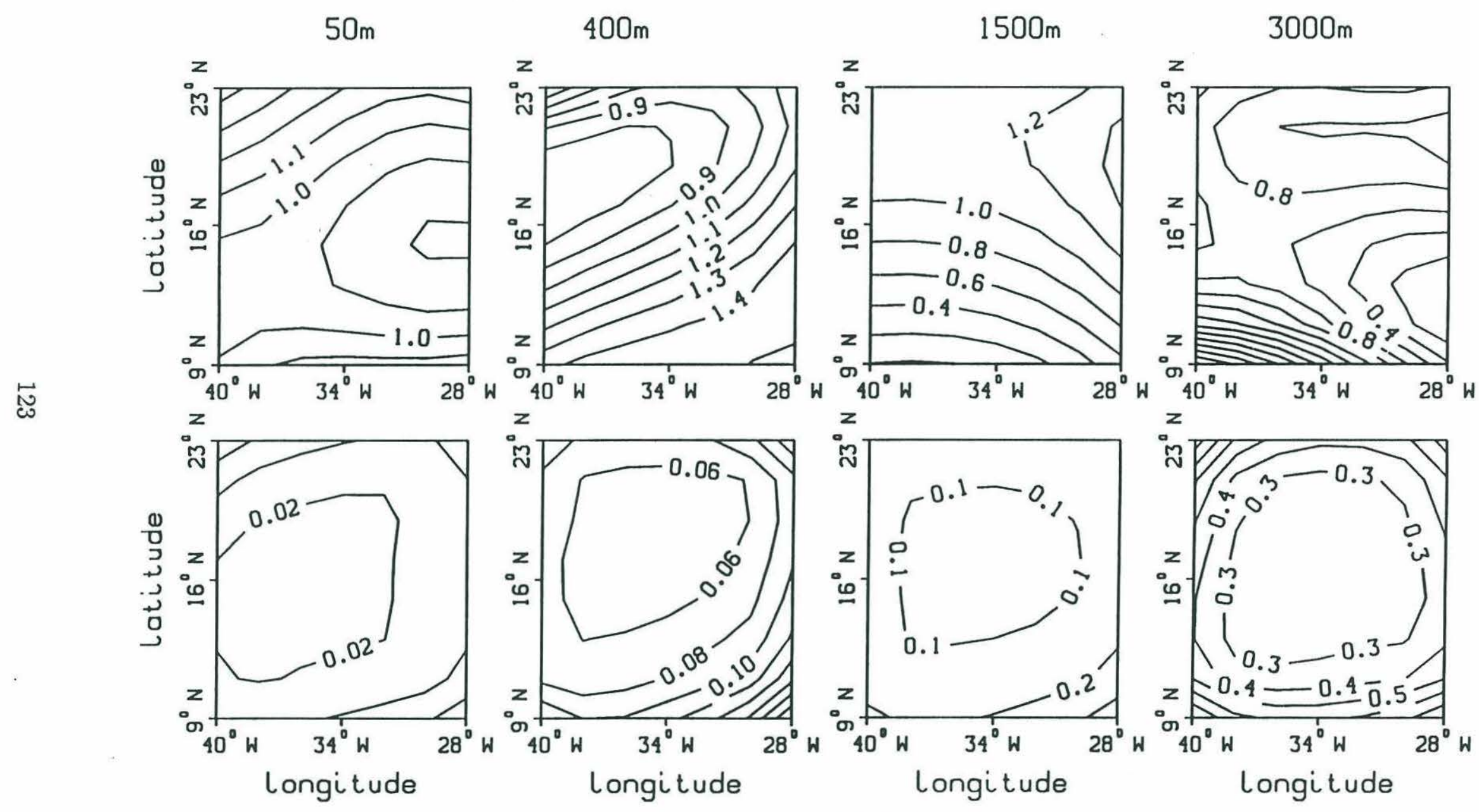

Fig. 3.26. (b) vertical diffusivity 

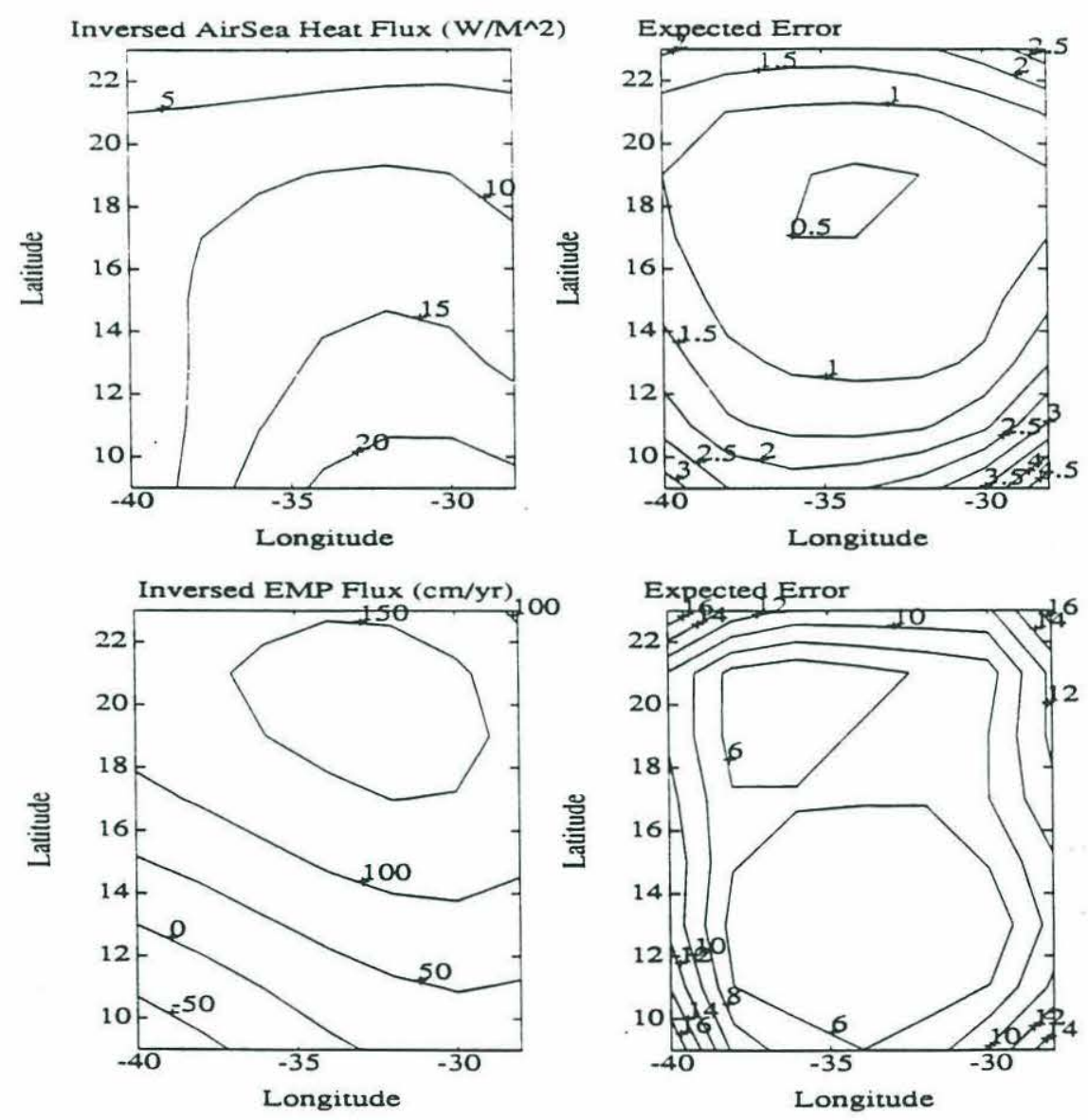

Fig. 3.27. Estimated air-sea heat $\left(W / m^{2}\right)$ and fresh water (EMP, $\left.\mathrm{cm} / y r\right)$ fluxes and their errors from experiment PAR II. 


\subsubsection{Discussion}

The turbulent diffusion coefficients, which were introduced to parameterize the sub-grid scale processes, may not be constant in space in the real ocean. In order to get better estimations for the parameters and in order to pose an overdetermined system in the inverse model, not all the parameters can be kept as point-wise unknowns. Parameterizations of some variables are necessary for this purpose as well as for the concern of computing cost. Also, different variables may require different parameterizations due to their different natures.

Vertical velocity $w$ is linked to the divergence of the horizontal velocity by the continuity equation. Small variations in the horizontal velocities $u, v$ can significantly affect the values of the vertical velocity. Therefore the vertical velocity distributions may scatter in space even though the distributions of the horizontal circulation are relatively smooth. As a result, small scale variations may be allowed in the parameterization of the vertical velocity. Whenever possible, vertical velocity should be kept as a point-wise unknown.

The distributions of turbulent diffusion coefficients in the ocean are barely known. Based on the present understanding of the horizontal and vertical diffusivities $A$ and $K$, smoothed versions of them should be acceptable. Therefore they can be parameterized as relatively low order polynomials or other functions with larger scale variations. Note that the steady state conservation equations for heat and salt are not exactly satisfied by the climatological mean data in the ocean, and the residuals (noises) in these equations may not be uniformly distributed in space. The previous experiments show that in the inverse model solutions, the minimization of the residual norm forces the non-white residuals in these equations into the 
"physical" terms-effectively the terms of diffusion, and thus modifies the "real" values of diffusion coefficients. The modifications are not uniform in space due to the inhomogeneity of the residuals. At extreme points where residuals are larger than diffusion terms, the real values of the diffusion coefficients may be totally disguised. Higher resolution parameterization of the diffusion coefficients may result in higher resolution solutions, but they may not necessarily represent the "real" distributions of the diffusion coefficients. Therefore high order polynomial parameterizations may not necessarily be better than low order ones. The lower order polynomial parameterization has similar effects as smoothing the noises in space: smoothing the estimated diffusion coefficients from a high order polynomial parameterization model would be similar to the inversion of a lower order polynomial parameterization model.

\subsection{SUMMARY AND CONCLUDING REMARKS}

In this chapter, the inverse model described in chapter 2 was applied to a noneddy resolving numerical GCM ocean to test how accurate the ocean circulations and diffusive parameters could be reproduced from the hydrographic data only by the inverse model.

Using the known physics and parameter values in this GCM ocean (which is generally unknown in the real ocean), the accuracy of the inverse model equations were estimated. It was shown that the horizontal circulations, especially the meridional components, are approximately in the thermal wind balance except in the surface mixed layer. The steady inverse model conservation equations for tracers are not exactly satisfied by the instantaneous potential temperature and salinity 
fields of the GCM ocean (integrated for 400 years). The temporal variation terms are quite large compared to the diffusion terms, especially in the deep layers. It was also noticed that the distributions of these terms are very scattered in space and somehow simulate the random data noises. The patterns of these distributions are very similar to those of the vertical velocity.

In the first experiment (the upper-level model), the inverse model was applied to a six-layer water column below the surface mixed layer and well above the ocean bottom (between $50 \mathrm{~m}$ and $1500 \mathrm{~m}$ ). In this water column the data "noises" in the steady conservation equations for heat and salt are the smallest relative to the advection and diffusion terms. The inverse model estimated horizontal circulations have the same flow patterns as those in the GCM ocean on all the six levels. The estimated values for the horizontal velocities are also consistent with the GCM ones within the estimated error bars on all the levels but the bottom one at $1250 \mathrm{~m}$, where the circulations are very weak. The estimated horizontal and vertical diffusion coefficients are $A=(1.08 \pm 0.02) \times 10^{7} \mathrm{~cm}^{2} / \mathrm{s}$ and $K=1.03 \pm 0.01 \mathrm{~cm}^{2} / \mathrm{s}$. The errors above are established on the $95 \%$ confidence interval. Although the deviations of the estimates from the GCM parameter values are very small, they are significantly above the estimated error bars. These significant biases are caused by the different physics of the inverse model and the numerical GCM ocean, as summarized in the previous paragraph. These effects were further seen in the experiments in a deeplayer model (with the vertical centers extend from $300 \mathrm{~m}$ to $2500 \mathrm{~m}$ ). An experiment allowing $A$ to be a different constant on different levels shows that the solutions for $A$ are consistent with the values of the numerical GCM parameter where the data "noises" are smaller than the diffusion terms, while the deviations are significant where the data "noises" are larger than the diffusion terms. 
It was noticed that the Ekman pumping velocities $\left(w_{e}\right)$ calculated from the wind stress are very similar to the estimated vertical velocities and those of the numerical GCM at the bottom of the surface layer (at $50 \mathrm{~m}$ ), but the values are not exactly the same. In dealing with the real ocean, one might try to use the available Ekman pumping velocity to constrain the solution for the vertical velocity at the bottom of the surface layer. Effects of this constraint on the inverse model solutions were examined. By enforcing $w$ at $50 \mathrm{~m}$ to be $w_{e}$, the estimated $A$ is $(1.43 \pm 0.07) \times 10^{7} \mathrm{~cm}^{2} / \mathrm{s}$ and $K$ is $1.11 \pm 0.04 \mathrm{~cm}^{2} / \mathrm{s}$. These estimates are further away from their "true" values. Even the estimated circulations differ from those in the previous case and those in the GCM ocean. A lesson from this experiment is that unless one has specific reason to believe that the $w$ at a certain depth should be $w_{e}$ (e.g., when the geostrophic velocity divergence related vertical velocity $w_{g}$ in the mixed layer is much smaller than $w_{e}$ ), the Ekman pumping constraint should be loosely applied. If the noise level of the wind stress is known, it should also be taken into account in choosing the weighting factor.

Geostrophic and thermal wind balances do not hold in the surface mixed layer. By adding the wind-driven Ekman transport in the conservation equation for mass, heat and salt, the air-sea heat and fresh water fluxes can also be estimated from the inverse model. The results showed that the estimates for these parameters are consistent with the values used in the GCM.

In the full-layer model which covers the whole water column from the ocean surface to the bottom with a total of 10 vertical levels, the results of a standard run, in which the parameters are treated the same as in the numerical GCM, were compared to the numerical GCM fields. It was shown that the estimated horizontal circulations are very similar to the GCM ones on all the 10 levels. On levels 4, 
5,6 and 7 at the depths of 300,500, 800 and 1250 meters, the consistency is not only in flow patterns, but also in the values of the horizontal velocities. On all the other levels, the values are close but yet the differences are significantly above the estimated errors in some regions. The inverse model solutions for the horizontal and vertical diffusion coefficients are $A=(1.09 \pm 0.02) \times 10^{7} \mathrm{~cm}^{2} / \mathrm{s}$ and $K=1.03 \pm 0.02 \mathrm{~cm}^{2} / \mathrm{s}$, which are very close to their "true" values but the small differences are significantly above the uncertainties. The significant biases are attributed to the discrepancy between the physics of the inverse model and the GCM.

The estimated vertical velocities from the standard run do not resemble the structures of the GCM vertical velocities. Instead they are very similar to the spatially smoothed version of the numerical GCM data. In fact, the estimates are statistically consistent with the 9-point smoothed GCM vertical velocities. The results of this experiment indicate that even if we do not know the data "noises", the inversions can still reveal the large scale structures of the ocean.

By utilizing the known temporal variation terms in the GCM ocean (which are usually unknown in the real ocean), we found totally consistent estimates for the horizontal and vertical diffusion coefficients $\left(A=(1.03 \pm 0.05) \times 10^{7} \mathrm{~cm}^{2} / \mathrm{s}\right.$ and $\left.K=0.99 \pm 0.02 \mathrm{~cm}^{2} / \mathrm{s}\right)$. In this case the inverse model solutions for the vertical velocity are also statistically consistent with those of the numerical GCM in detailed small scale structures (i.e. the non-smoothed "raw" data fields). As the formulation and accuracy of the dynamic equations have not been changed, the solutions for the horizontal circulations did not change very much. But in the increased estimated errors, the estimated circulations are statistically consistent with the GCM ones on all the levels. 
After noticing the existence of the data "noises" in the steady state heat and salt conservation equations, experiments on the parameterization of some variables were carried out. The results showed that the solutions for velocities $\overrightarrow{\mathbf{u}}, w$ are relatively robust, while those for the diffusivities $A, K$ (especially for $A$ ) are sensitive to the parameterizations. Parameterizing $A, K$ and air-sea fluxes as (third order) polynomials while keeping other variables (the velocities) as grid-point unknowns results in plausible solutions for all the variables on all the levels except the $A$ on the deepest three levels, where "noise" is larger than the diffusion terms.

Although the numerical model results used in this work are from a non-eddy resolving model, data "noise" does exist in the steady state conservation equations for heat and salt. Therefore the above discussions may also give us some guidance in dealing with the real ocean. However, testing the inverse model in an eddy-resolving GCM ocean would be more relevant to the application of the inverse model in the real ocean. 


\section{Chapter 4. APPLICATION OF THE INVERSE MODEL IN AN EDDY-RESOLVING NUMERICAL GCM OCEAN}

\subsection{INTRODUCTION}

In Chapter 3, the application of the inverse model to a non-eddy resolving numerical GCM ocean was discussed. It was shown that, from the numerical GCM "hydrographic" data, and under appropriate parameterization, the inverse model is quite capable of recovering the "true" ocean circulations, vertical velocities, horizontal and vertical diffusion coefficients, as well as the air-sea heat and fresh water fluxes of the non-eddy resolving numerical GCM ocean. The very small discrepancies among the values of the inversion and the values of the numerical GCM data were accounted for by the differences of the physics and assumptions used in the inverse model and those used in the numerical GCM.

However, the real oceans are full of eddy activities and characterized by seasonal variations (e.g., seasonal variation of wind forcing and air-sea heat and fresh water fluxes). The inverse model used in this work, as with most other inverse models, was formulated to study the circulations and diffusion processes (tracer balances) in the real ocean. Thus it is more relevant to test the inverse model in a more realistic ocean, namely an eddy-resolving numerical GCM ocean (as there are not enough data available to test the inverse model in the real ocean).

The climatological mean ocean circulation, which is one of the major objectives of the inverse model, is theoretically defined as the ensemble mean of an infinitively long time series of ocean circulation. As the direct measurement of the ocean circulation, especially in the deep ocean, is more difficult and expensive than 
the direct measurement of the hydrographic data, the climatological mean ocean circulations (especially those in large scale) are usually deduced from the time-mean, and in many cases also spatially smoothed, hydrographic data.

Among the tools used to deduce oceanic circulation from hydrographic (and other tracer data) are the inverse models. But are the results from the inversion of the spatially smoothed time-mean hydrographic data what we are after? (e.g., are the deduced circulations the spatially smoothed climatological time-mean ocean circulations?) And how close are the inverse model estimates to their true values?

There are practically two aspects in answering the first of the above questions: one is the representativeness of the limited time means of the hydrographic data to their true climatological means; and the other is the correct interpretation of the inverse model results of the spatially smoothed time-mean hydrographic data.

To the first aspect, the time means of a collection of hydrographic data over a limited time period are generally not the climatological means (over a long time period), especially in the thermocline. Climatological hydrographic data are usually collections of individual cruise data made in different seasons of different years. The combination and time average of these individual observations could be biased from the true climatological means if they do not evenly span the different seasons of the years. For example, if a data set used to compose a climatological atlas consists of more observations made in summer than in winter, the time mean of them might result in a higher temperature field than the true climatological mean in the thermocline. Besides the observational bias in time, the spatial coverage of the hydrographic stations is still not dense enough in the world oceans, especially in the deep part, at the present time. Most of the so-called climatological atlases 
(e.g., Levitus, 1982) are produced by using some kind of objective analysis. In large domains where observations are not available, the "interpolated" data might be biased from their true values. The improvement of the limited time means of the hydrographic data towards their true climatological mean values can and would be most effectively achieved by more observations (in both time and space) in the future.

Even if we have a reliable climatological hydrographic data set, the question of whether the inverse estimates for the parameters (circulations, eddy diffusion coefficients etc.) from the spatially-smoothed time means can closely represent the spatially-smoothed climatological mean parameter values still exists. This problem arises from the fact that, theoretically, the orders of inverse and time mean as well as spatial smoothing are not commutable. Tziperman (1988) tried to answer the time averaging issue by comparing the inversion of a time averaged data set (which consists of six summer cruises in the eastern Levantine Basin of the Mediterranean Sea) and the average of the inversions of the six individual cruises. He concluded that the circulations from the two methods are surprisingly similar, but not the diffusion coefficients. It should be pointed out that, first of all, the average of six realizations is hardly a climatological mean. Also, the steady state equations (e.g., the conservation equations for heat) used in his model might not be appropriate for the inversion of the individual instantaneous cruise data. (For example, the time variation term may also be important.)

When spatial smoothing is used, the issue becomes more complicated. Unlike the time mean, which is taken over the whole sampling time period (and thus the time means are independent of time in the time period concerned), the spatial smoothing is done locally (e.g., a Gaussian smoothing with radius of 200 kilome- 
ters), and thus both the spatially smoothed fields and the perturbations from the smoothed fields are functions of space. The perturbations of the data from the spatially smoothed fields can introduce more terms in the conservation equations (of mass, heat, salt, etc), and if they are neglected in the model, they serve as extra error sources for the equations for the smoothed data.

The main objective of this chapter is to answer the question of whether the application of the inverse model on the spatially smoothed time-mean hydrographic data can reveal the spatially smoothed time-mean ocean circulations and eddy diffusive parameters in different grid spacing. The inverse model will be tested in an eddy-resolving numerical GCM ocean described in the following section.

\subsection{THE NUMERICAL GCM OCEAN}

The eddy-resolving numerical GCM ocean used in this chapter was generated by running the GFDL model with the following parameter values and configurations (F. Bryan, personal communication). The horizontal resolution in this run, to explicitly include the hydrodynamic instability process responsible for eddy formation, is $2 / 5^{\circ}$ longitude by $1 / 3^{\circ}$ latitude which gives equal grid spacing in north-south and east-west directions at $34^{\circ}$ latitude. Vertically there are 30 levels, with a spacing of $35 \mathrm{~m}$ at the surface and smoothly stretching to $250 \mathrm{~m}$ by $1000 \mathrm{~m}$ depth. Below 1000 $\mathrm{m}$ the vertical grid spacing is a constant $250 \mathrm{~m}$. The computational domain of the numerical model is the North Atlantic basin from $15^{\circ} \mathrm{S}$ to $65^{\circ} \mathrm{N}$ latitude including the Caribbean Sea and Gulf of Mexico, but excluding the Mediterranean Sea (Fig. 4.1). As mentioned in Chapter 2, instead of the conventional Laplacian dissipation, the horizontal dissipation mechanism is a highly scale-selective biharmonic (fourth- 
order derivative) operator, with coefficients of $-1.0 \times 10^{19} \mathrm{~cm}^{4} / \mathrm{s}$ for momentum and of $-2.5 \times 10^{19} \mathrm{~cm}^{4} / \mathrm{s}$ for tracers. The vertical dissipation mechanism is the more traditional second-order Laplacian operator with constant coefficients of 10 $\mathrm{cm}^{2} / \mathrm{s}$ for momentum and $0.3 \mathrm{~cm}^{2} / \mathrm{s}$ for tracers. The model is forced with seasonally varying climatological wind stress and surface heat and fresh water fluxes.

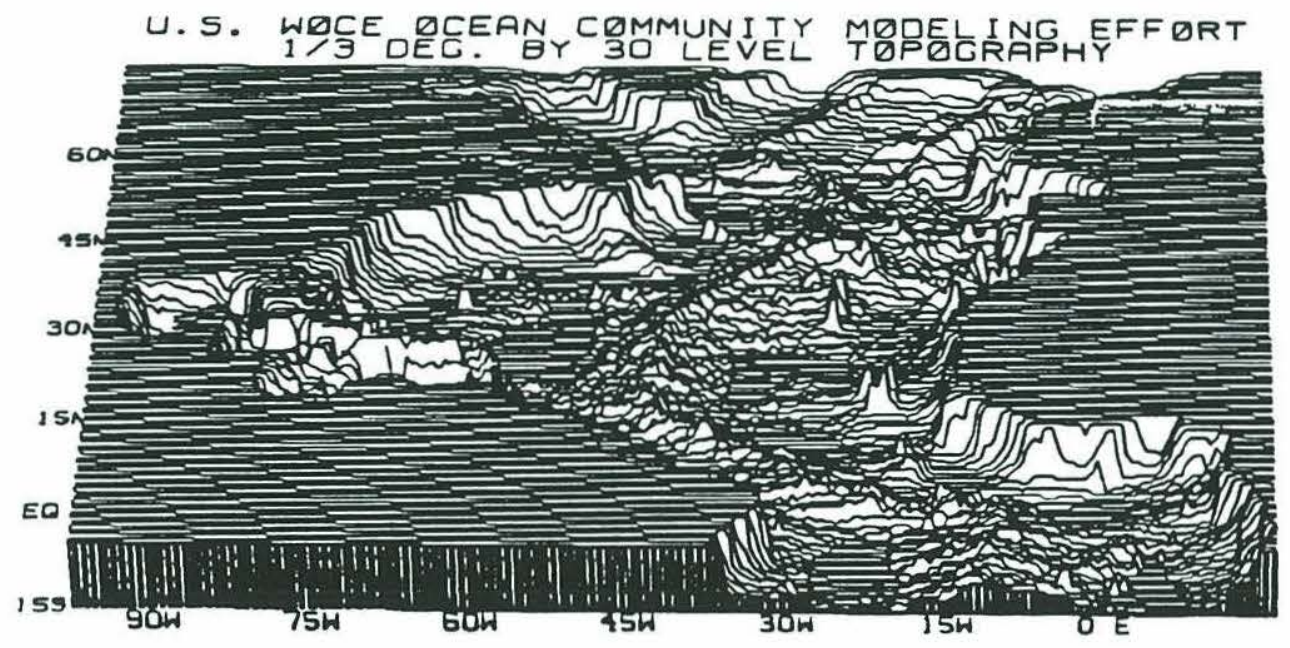

Fig. 4.1. Three-dimensional perspective view of the bottom topography used in the EGCM. (Bryan et al, 1989).

The evolving model solution was sampled at three-day time interval during the final five years of the simulation. The time means of the five-year, 600 -sample time series are used as the climatological means for the inverse model. In order to analyze the inverse model results, it is necessary to compute the errors for the 5-year time means.

\section{Estimating the Means and Errors for the Means}

There are rigorous ways to determine the means (either time means or spatial means) and the errors of the means. One of them is the Gauss-Markov theorem (e.g.. Bretherton et al., 1976; Wunsch, 1989). Let the measurements of a physical 
quantity to be $Y=\left\{y_{i}\right\}$ and suppose that they are made up of an unknown large scale mean $m$, plus deviations $\Theta=\left\{\theta_{i}\right\}$ from that mean:

$$
D m+\Theta=Y, \quad D=[1,1, \ldots 1]^{T} .
$$

We seek a best estimate $\hat{m}$ of $m$. If the statistics, i.e., the covariance of data fluctuations $R_{n n}=\left\langle\Theta \Theta^{T}\right\rangle$, is known, the Gauss-Markov theorem can be used to determine the means and the expected errors of the means:

$$
\begin{aligned}
\hat{m} & =\frac{1}{1 / m_{0}^{2}+D^{T} R_{n n}^{-1} D} D^{T} R_{n n}^{-1} Y \\
E & =\frac{1}{1 / m_{0}^{2}+D^{T} R_{n n}^{-1} D}
\end{aligned}
$$

where $m_{0}$ is an a priori estimate of the mean. In our current data set, the statistics like $R_{n n}$ are unknown, and we must search for other approaches to estimate the means and the errors of the means.

One way the means can be estimated is taking the sample means:

$$
\bar{T}=\frac{1}{N} \sum_{t=1}^{N} T_{t}
$$

where $N=600$ in our case. Assume that

$$
<T_{t}>=\mu ; \quad \operatorname{var}\left(T_{t}\right)=\sigma^{2} ; \quad \operatorname{cov}\left(T_{t}, T_{t+\tau}\right)=R(\tau)=\sigma^{2} \rho(\tau) .
$$

Then the variance of the mean $(\bar{T})$ can be estimated as (e.g., Priestley, 1989)

$$
\begin{aligned}
\sigma_{\bar{T}}^{2} & =\left\langle(\bar{T}-\mu)^{2}>=\frac{1}{N^{2}} \sum_{s=1}^{N} \sum_{t=1}^{N} \operatorname{cov}\left(T_{s}, T_{t}\right)=\frac{\sigma^{2}}{N^{2}} \sum_{s=1}^{N} \sum_{t=1}^{N} \rho(t-s)=\frac{\sigma^{2}}{N^{2}} \sum_{s=1}^{N} \sum_{\tau=1-s}^{N-s} \rho(\tau)\right. \\
& =\frac{\sigma^{2}}{N} \sum_{\tau=-(N-1)}^{N-1}\left(1-\frac{|\tau|}{N}\right) \rho(\tau)=\frac{\sigma^{2}}{N}\left[\rho(0)+2 \sum_{\tau=1}^{N-1}\left(1-\frac{\tau}{N}\right) \rho(\tau)\right] \\
& =\frac{\sigma^{2}}{N}\left[1+2 \sum_{\tau=1}^{N-1}\left(1-\frac{\tau}{N}\right) \rho(\tau)\right] \approx \frac{\sigma^{2}}{N}\left[1+2 \sum_{\tau=1}^{N-1} \rho(\tau)\right]
\end{aligned}
$$


where the approximation is taken for large sample size (large N). If the data are totally uncorrelated (white noise), we have $N$ independent samples and the variance of the mean is simply $\sigma^{2} / N$ (the degree of freedom, or $d f$, equals to $N$ ).

In some works the variance of the mean is also expressed in terms of the degree of freedom:

$$
\sigma_{\bar{T}}^{2}=\sigma^{2} / d f
$$

and thus from the above expressions the effective $d f$ in terms of the auto-correlation is

$$
d f=\frac{N}{1+2 \sum_{\tau=1}^{N-1}\left(1-\frac{\tau}{N}\right) \rho(\tau)} \approx \frac{N}{1+2 \sum_{\tau=1}^{N-1} \rho(\tau)} .
$$

In terms of the sampling time period $P$ and the integral time scale $\Gamma$, the $d f$ is defined as

$$
d f=\frac{P}{2 \Gamma}=\frac{N \Delta t}{2 \Gamma} .
$$

Comparison of the above two expressions for the $d f$ yields the integral time scale in terms of the auto-correlation coefficients:

$$
\Gamma=\left[\frac{1}{2}+\sum_{\tau=1}^{N-1}\left(1-\frac{\tau}{N}\right) \rho(\tau)\right] \cdot \Delta t \approx\left[\frac{1}{2}+\sum_{\tau=1}^{N-1} \rho(\tau)\right] \cdot \Delta t
$$

which is the Simpson's rule version of the integral of $\rho(\tau)$ with respect to $\tau$.

In practice, not all the auto-correlation coefficients are significantly different from zero, and the summation over $r$ in determining the $\sigma_{\bar{T}}^{2}, d f, \Gamma$ generally should 
not be carried out to the very end (to N-1) (where $\rho(\tau)$ is usually meaningless). Determining the upper limit of the summation is a difficult task. This usually involves the analysis of the variances of the auto-correlation coefficients, which itself is a complicated subject. Detailed discussion on this subject can be found in Priestley (1989), or Bendat and Piersol (1986). Under the assumption that $\left\{T_{t}\right\}$ is stationary up to order four and is a Gaussian process, and $N$ is large, the variances of the $\hat{\rho}(\tau)$ can be estimated as (e.g., Priestley, 1989)

$$
\begin{aligned}
& \operatorname{var}\{\hat{\rho}(\tau)\} \\
\sim & \frac{1}{N} \sum_{m=-(N-\tau-1)}^{N-\tau-1}\left[\rho^{2}(m)+\rho(m+\tau) \rho(m-\tau)+2 \rho^{2}(\tau) \rho^{2}(m)-4 \rho(\tau) \rho(m) \rho(m-\tau)\right] \\
\sim & \frac{1}{N} \sum_{m=-\infty}^{\infty}\left[\rho^{2}(m)+\rho(m+\tau) \rho(m-\tau)+2 \rho^{2}(\tau) \rho^{2}(m)-4 \rho(\tau) \rho(m) \rho(m-\tau)\right]
\end{aligned}
$$

For finite $N$, the distribution theory of the sample auto-correlation coefficients is extremely complicated. But for approximate large sample (large $N$ ) tests and confidence intervals, the asymptotic normality of the $\{\hat{\rho}(\tau)\}$ may be used.

Some examples of the auto-correlation coefficients (at $577 \mathrm{~m}$ depth) are shown in Fig. 4.2 together with their $95 \%$ confidence intervals. The maximum lag shown is 300 , and with $\mathrm{N}=600, \mathrm{~N}-\tau$ (the number of samples used in computing the $\rho(\tau))$ are generally larger than 300. For lag larger than 300, the correlation coefficients are indistinguishable from zero. For temperature $T$ at $577 \mathrm{~m}$, the autocorrelation coefficients have no significance after about $20 \times 3.04$ days (Fig. 4.2a). For salinity $S$ at the same depth, the auto-correlation coefficients are essentially indistinguishable from zero after about $20 \times 3.04$ days except at the lag of about $125 \times 3.04$ days (Fig. 4.2 b), where they are marginally positive with significance. For the zonal velocity $u$, the auto-correlation coefficients are insignificant after about $20 \times 3.04$ days except at the lag of about $40 \times 3.04$ days (Fig. 4.2 c), where they are 
marginally negative. The auto-correlation coefficients for other variables and at other depths have similar features.

Due to the complicated distributions of the auto-correlation coefficients, it is difficult to determine the upper limit of the summation in determining $\sigma_{\bar{T}}^{2}, d f$, and $\Gamma$. Since the auto-correlation coefficients are generally insignificant after they cross the first zero line (from unity), we decided to carry out the summations from $\rho=1$ to the first $\rho=0$. For variables which have significant positive correlations beyond this point (the first zero correlation), like $S$ in Fig. 4.2b, our method under-estimates the integral time scales and the errors of the means (over-estimates the degree of freedom). In general, for this data set, the auto-correlations have little significance after the first zero correlation. Also, the errors of the means are proportional to the square root of the integral time scales (inversely proportional to the square root of the degree of freedom). Thus we believe that our method gives reasonable estimates of the typical errors for the means although it is somewhat ad hoc. 
(a) 5-year (Y20-Y24) Time series from GCM. T@ $(34 \mathrm{~W}, 17.5 \mathrm{~N}, 577 \mathrm{~m})$
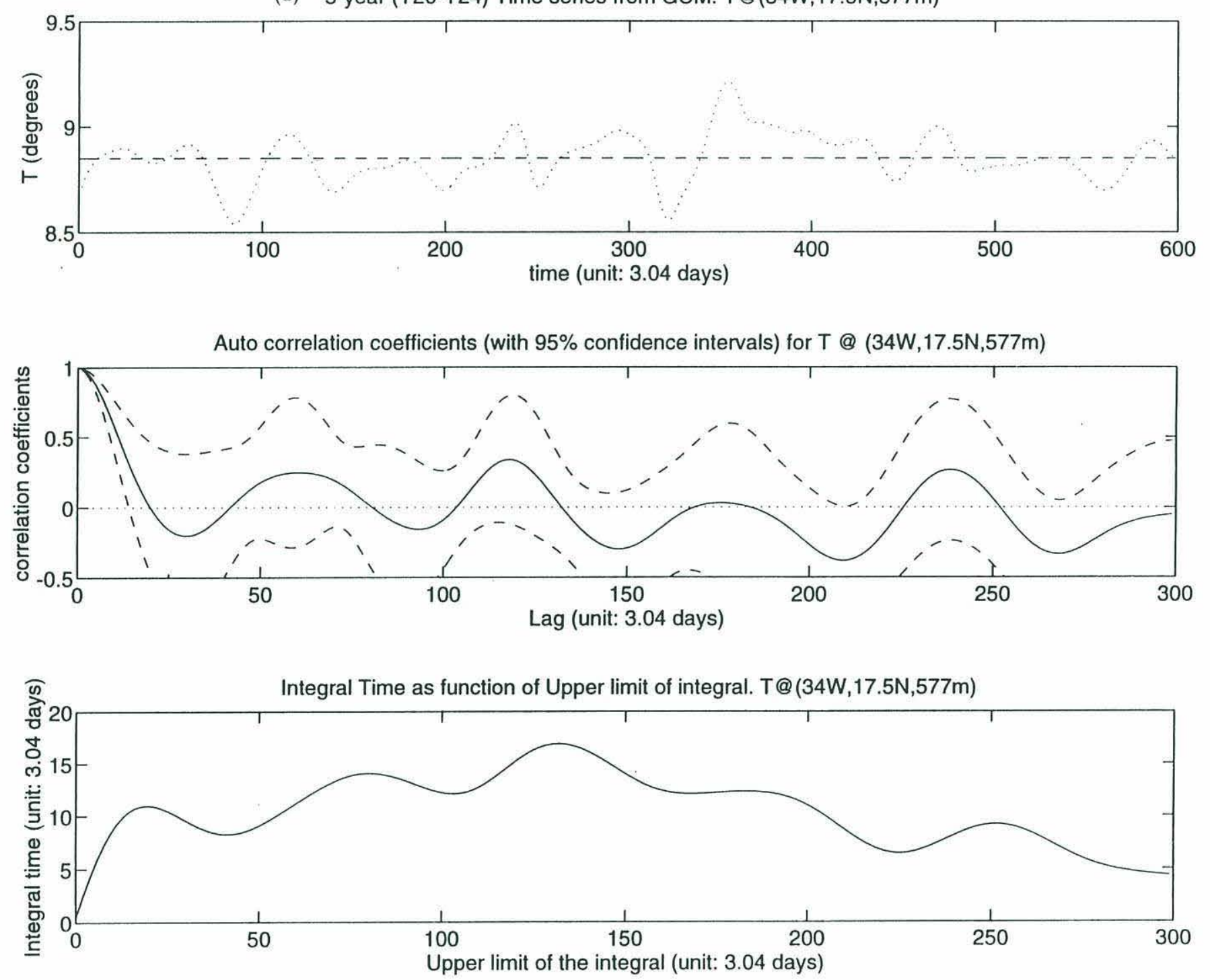

Fig. 4.2. The 5-year, 600 samples (top), their auto-correlations with $95 \%$ confidence intervals (middle), and the integral time scales (bottom) for (a) temperature; (b) salinity; (c) zonal velocity. 

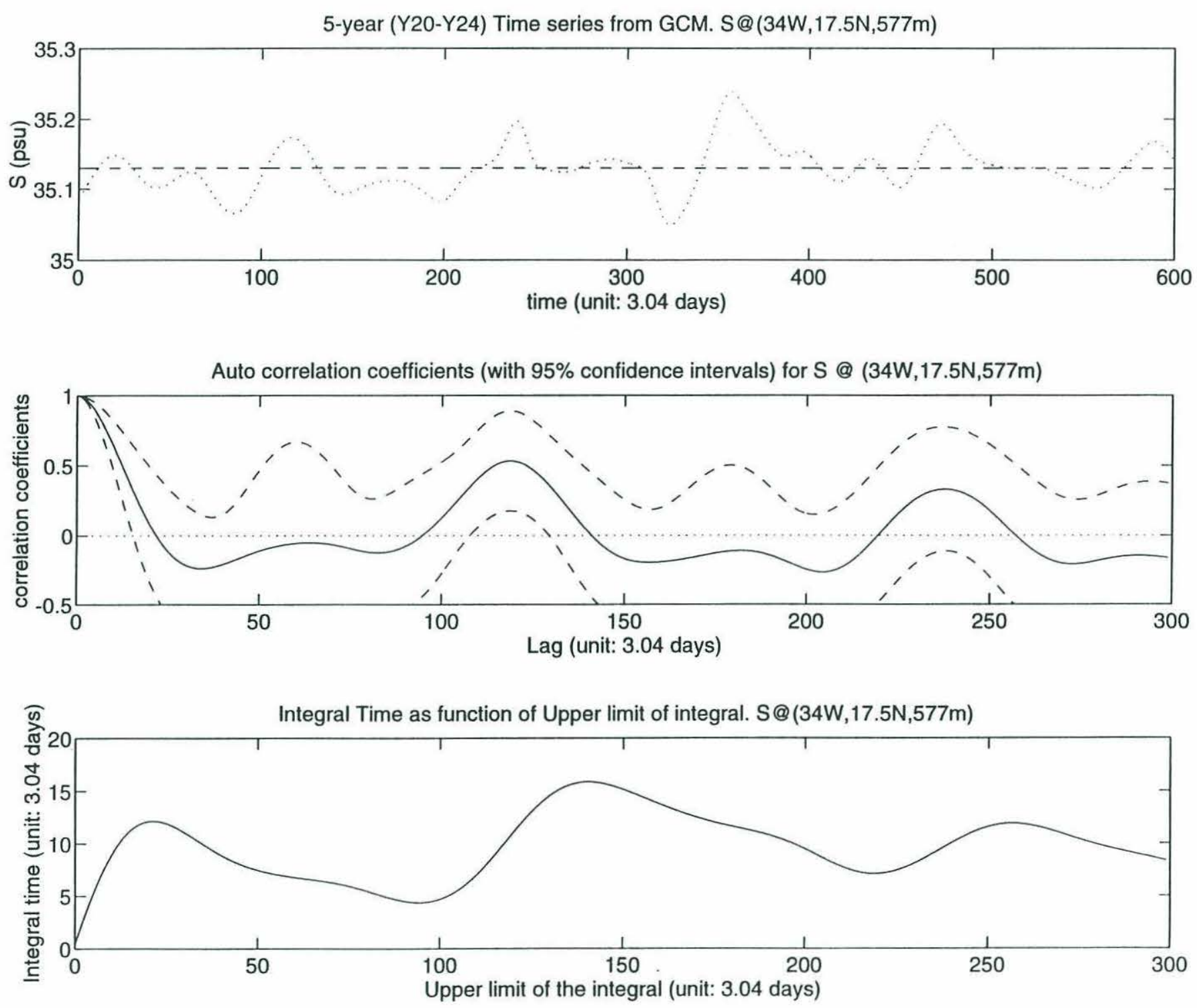

Fig. 4.2 (b) 

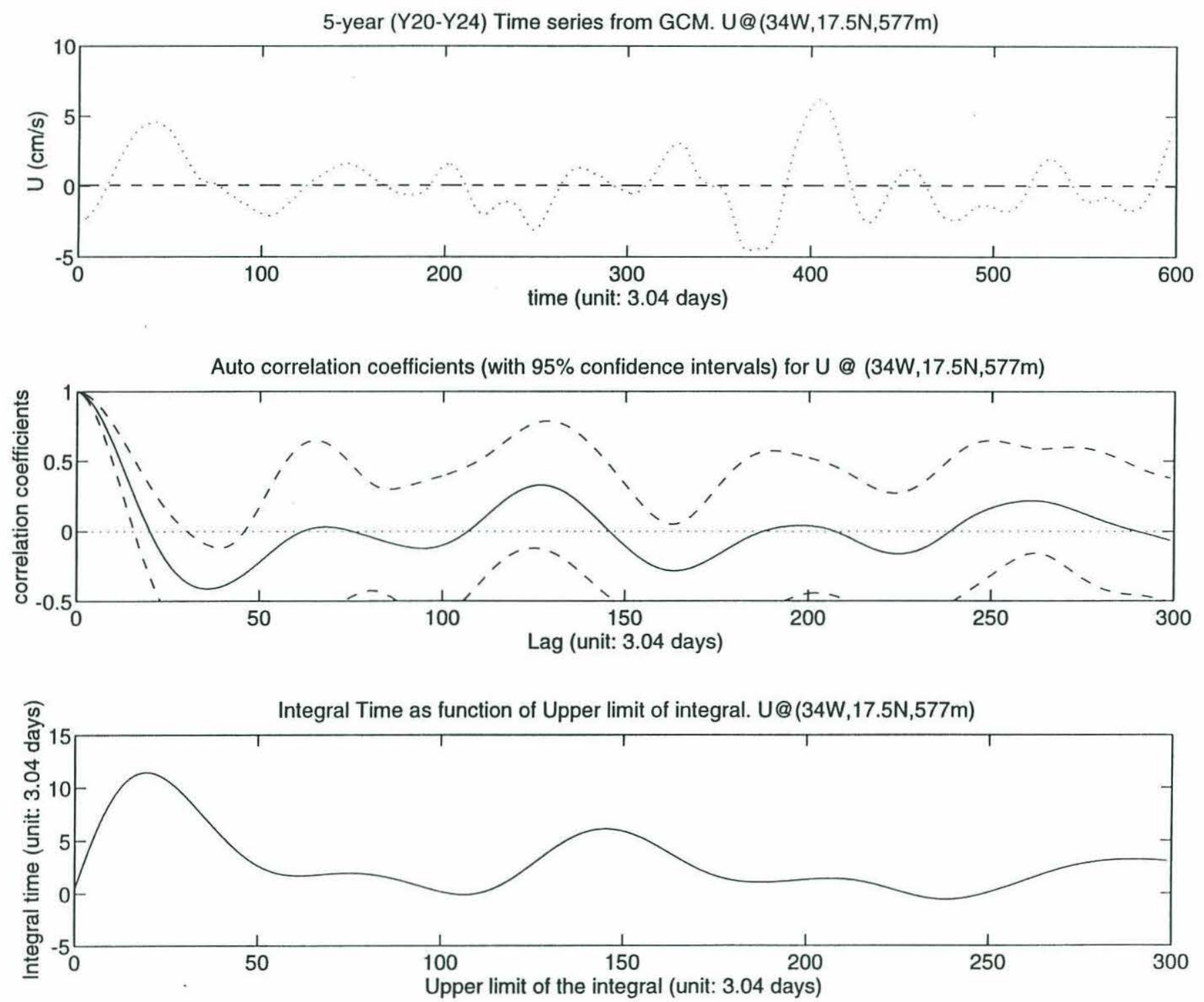

Fig. 4.2 (c) 
Table 4.1 Integral time scales (days) for different variables at various depths at $\left(34^{\circ} \mathrm{W}\right.$, $\left.17.5^{\circ} \mathrm{N}\right)$.

\begin{tabular}{|c|c|c|c|c|c|c|c|}
\hline Depth (meters): & $92 \mathrm{~m}$ & $233 \mathrm{~m}$ & $370 \mathrm{~m}$ & $577 \mathrm{~m}$ & $901 \mathrm{~m}$ & $1375 \mathrm{~m}$ & $1875 \mathrm{~m}$ \\
\hline$\Gamma$ for $\mathrm{T}$ : & 30 & 28 & & 35 & 40 & 70 & 50 \\
\hline$\Gamma$ for $S$ : & 40 & 30 & & 40 & 58 & 230 & 125. \\
\hline$\Gamma$ for $u$ : & 45 & & & 35 & 37 & 39 & 42 \\
\hline$\Gamma$ for $v$ : & 18 & & & 22 & 22 & 22 & 22 \\
\hline$\Gamma$ for $w$ : & 17 & & & 18 & 20 & 20 & 19 \\
\hline$\Gamma$ for $u T^{\prime}:$ & & 18 & 22 & 27 & 25 & 35 & 26 \\
\hline$\Gamma$ for $v T^{\prime}:$ & & 15 & 17 & 18 & 18 & 22 & 22 \\
\hline$\Gamma$ for $w T^{\prime}:$ & & 15 & 15 & 15 & 14 & 17 & 17 \\
\hline$\Gamma$ for $u S^{\prime}:$ & & 20 & 23 & 24 & 24 & 35 & 32 \\
\hline$\Gamma$ for $v S^{\prime}:$ & & 15 & 15 & 17 & 20 & 22 & 22 \\
\hline$\Gamma$ for $w S^{\prime}:$ & & 14 & 15 & 15 & 16 & 16 & 20 \\
\hline
\end{tabular}

The integral time scales defined above for different variables at different depth at $\left(34^{\circ} \mathrm{W}, 17.5^{\circ} \mathrm{N}\right)$ are shown in Table 4.1. This table, first of all, shows that the integral time scales of the numerical GCM ocean are longer than the typical values of the real ocean (of order 10 days). Also, this table shows that the correlation time scales of the tracers $\mathrm{T}$ and $\mathrm{S}$ ) are typically larger than those of the velocities. The bluer spectra of velocity than those of tracers are consistent with Owens' (1979) low modes analysis. For the velocities themselves, it is shown that the zonal component has a longer correlation time than the meridional component, which in turn has a longer correlation time scale than the vertical velocity. The longer integral time 
scales in zonal velocity than in meridional velocity are expected on a $\beta$-plane, and these behaviors were also found in data (e.g., Spall et al, 1993, Spall, 1994). This behavior is also true for the components of the eddy fluxes. It is also noticed that the integral time scales for the eddy heat and salt fluxes are approximately the same, and they are closer to those of the velocities than to those of the tracers (temperature and salinity).

With the $95 \%$ confidence intervals, the errors of the time-mean zonal velocity (u) are in the order of $1.0 \mathrm{~cm} / \mathrm{s}$ (at upper levels) to $0.4 \mathrm{~cm} / \mathrm{s}$ (at greater depths), those of the meridional velocity (v) in the order of $0.6 \mathrm{~cm} / \mathrm{s}$ (at upper levels) to 0.3 $\mathrm{cm} / \mathrm{s}$ (at greater depth), while those for the vertical velocity (w) are in the order of $8 \times 10^{-4} \mathrm{~cm} / \mathrm{s}$ (at upper depths) to $18 \times 10^{-4} \mathrm{~cm} / \mathrm{s}$ (at greater depths). The statistical errors of the tracers are quite small: $\sigma_{\bar{T}} \sim 0.2^{\circ} \mathrm{C}$ (at upper levels) to $0.02^{\circ} \mathrm{C}$ (at greater depths). More information on the GCM fields and their errors can be found in later sections in the analysis of the inverse model results.

\subsection{INVERSION OF THE TIME-MEAN FIELDS}

\subsubsection{Introduction}

In this section, we will see how the inverse model functions in the time-mean fields of the numerical EGCM ocean. The input for the inverse model are the time means of temperature and salinity (as well as the derived density fields). The inverse model results will be compared to the time mean fields of the GCM circulations and vertical velocities, as well as to the eddy diffusion coefficients directly computed from the eddy fluxes in the GCM ocean. 
In the inversion performed in this section, the finite grid resolution is chosen to be the same as EGCM one, leaving the issue of spatial smoothing and subsampling to the next section. With this fine grid resolution $\left(2 / 5^{\circ}\right.$ longitude by $1 / 3^{\circ}$ latitude), and limited by computer resources, we face the dilemma of doing the inversion either in a larger horizontal domain and a shallow water column (less vertical levels), or in a smaller horizontal domain and a deeper water column (more vertical levels). For example, if one wants to cover a horizontal area of $6^{\circ}$ by $6^{\circ}$ and vertically from $133 \mathrm{~m}$ to $1875 \mathrm{~m}$ (totaling of 13 vertical levels), the inversion (the SVD for a more complete analysis) requires at least 205 mega-words of memory, which is well above the capacity (of 60 mega-words) of the CRAY super computers at both MIT and NCAR.

Experiments show that the inverse model results are sensitive to the vertical levels and water column thickness (one example will be reported in section 4.4.4), but relatively insensitive to the size of horizontal domain (also see, Hogg, 1987), if it is not too small (e.g., to allow the T, S gradients, thus the equation coefficients, to be well defined). Therefore it was decided to run the inverse model in a smaller horizontal domain but with more vertical levels. The domain is chosen extending from $34.8^{\circ} \mathrm{W}$ to $31.6^{\circ} \mathrm{W}$ and from $16.17^{\circ} \mathrm{N}$ to $19.17^{\circ} \mathrm{N}$, and vertically from $133 \mathrm{~m}$ to $1875 \mathrm{~m}$ depth, resulting in a grid number of $8 \times 9 \times 13$. 


\subsubsection{Accuracy of the Inverse Model in the Time Mean Fields of the EGCM Ocean}

\section{Continuity Equation:}

In the instantaneous fields of the EGCM ocean, the continuity equation is exactly satisfied (as a matter of fact, the values of the vertical velocity $w$ are diagnostically computed from the divergence of the horizontal velocity through the continuity equation). As the orders of taking the time mean and orders of taking spatial divergence are commutable, we expect that the continuity equation is also exactly satisfied by the time means of the velocities, and numerically indeed it is.

\section{Thermal Wind Relation}

The instantaneous horizontal velocities are calculated from the full non-linear prognostic momentum equations in the EGCM, with horizontal biharmonic dissipations. As in the real ocean, we do not expect that the time means of the GCM circulations will satisfy the thermal wind relation exactly, but we do expect that they are in good agreement (otherwise the assumptions of the inverse model would be violated and we would be using an incorrect model).

From the available time mean fields of the GCM data, the absolute velocity shears between two depths can be directly computed (denoted by UrGCM, etc.). On the other hand, the thermal wind shear (denoted by UrDyn, etc.) can be computed from the time-mean hydrographic data of the GCM ocean. Some of these calculations are shown in Fig. 4.3. In this figure, the first column is the UrGCM, the second column is the UrDyn, and the third column is their imbalance, which 
signifies the closeness/difference of the time mean circulation to/from the thermal wind balance. Note that the contour intervals in the third column are $1 / 10$ of those in the first and second columns. It can be seen that the time mean numerical GCM circulations are in good agreement with the thermal wind balance. In terms of the ratios $R_{u}$ and $R_{v}$ defined in Chapter 3 (eq.(3.1) and eq.(3.2)), the relative imbalances (deviations from thermal wind relation) are typically around $6 \%$ in the zonal direction and $4 \%$ in the meridional direction (Table 4.2). These numbers are smaller than those in Chapter 3, which means the time mean circulations here are closer to thermal wind balance. It is also noticed that, as in the non-eddy resolving GCM ocean (Chapter 3), the meridional velocity is closer to thermal wind balance than the zonal component.

Table 4.2 Relative imbalances between the time-mean absolute velocity shears and the thermal wind shears.

\begin{tabular}{lllllllllllll} 
depth range $(\mathrm{m})$ \\
$\quad 133-180-233-295-370-463-577-722-901-1125-1375-1625-1875$ \\
$R_{u}$ & 0.04 & 0.04 & 0.05 & 0.06 & 0.07 & 0.09 & 0.08 & 0.02 & 0.04 & 0.07 & 0.07 & 0.08 \\
$R_{\nu}$ & 0.03 & 0.03 & 0.03 & 0.04 & 0.06 & 0.05 & 0.05 & 0.06 & 0.03 & 0.04 & 0.05 & 0.04 \\
\hline
\end{tabular}




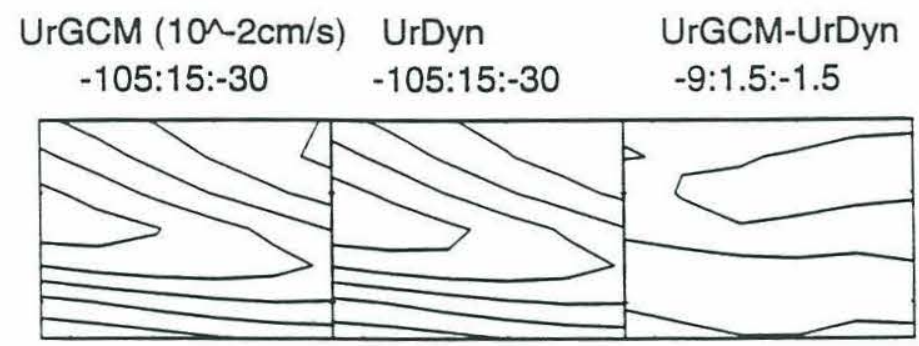

$133.19--179.52 m$

$-105: 15:-30 \quad-105: 15:-30 \quad-9: 1.5:-1.5$

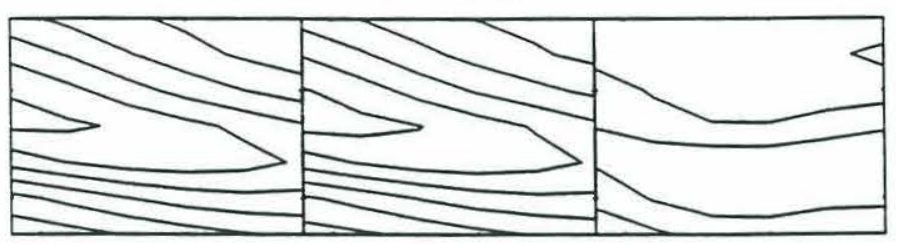

$179.52-232.595 m$

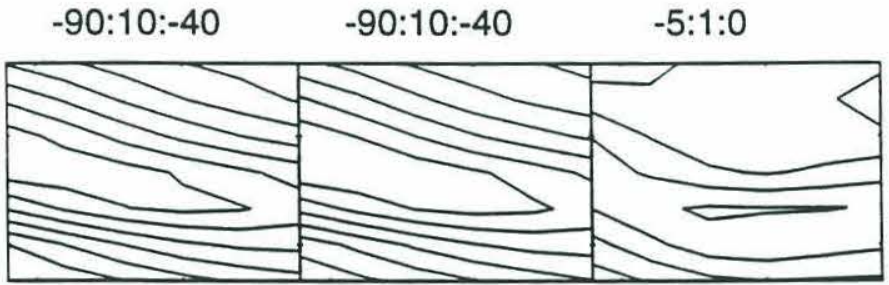

$232.595-295.025 m$

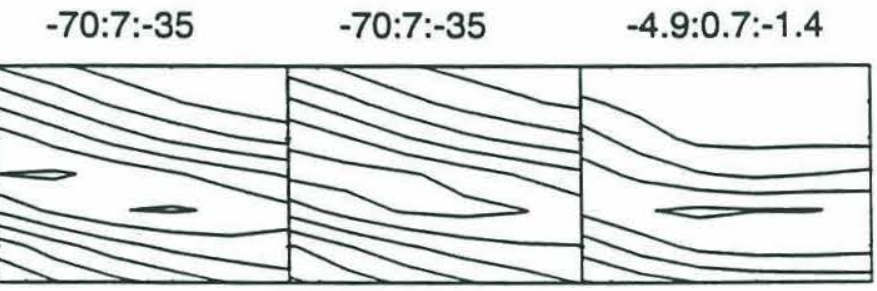

295.025--370.21m

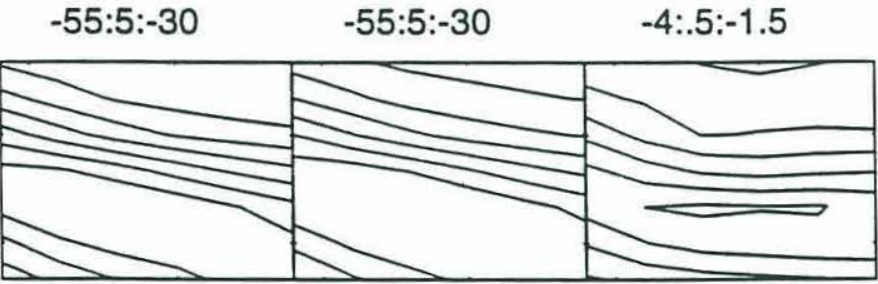

$370.21--462.505 m$

$-45: 10: 5$

$-45: 10: 5$

$-4.5: 1: .5$

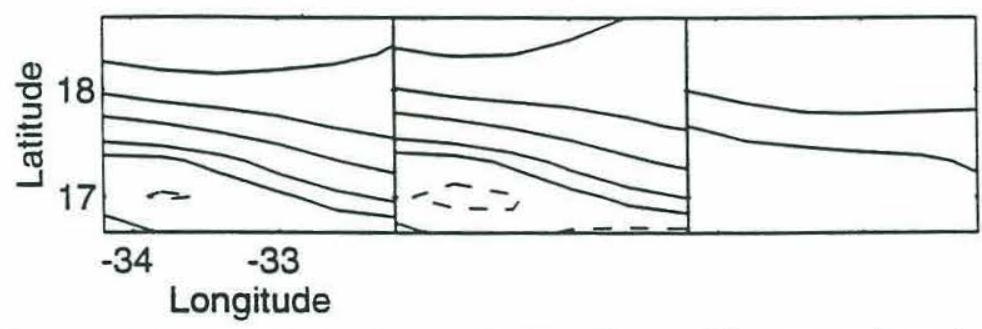

$462.505-577.365 m$

Fig. 4.3. EGCM absolute velocity shears (the 1st column), thermal wind shears (the 2nd column), and their differences (the 3rd column). The numbers at the tops are the minimum contour values: contour intervals : maximum contour values. 


\section{Conservation Equation for Tracers ( $\mathrm{T}$ and $\mathrm{S})$}

The tracer concentrations ( $\mathrm{T}, \mathrm{S}$, etc.) in the numerical GCM ocean are predicted by the prognostic conservation equation with biharmonic horizontal dissipation:

$$
\frac{\partial T}{\partial t}+\nabla \cdot(\vec{u} T)+\frac{\partial(w T)}{\partial z}=A_{m} \nabla^{4} T+K_{m} \frac{\partial^{2} T}{\partial z^{2}} \quad .
$$

where $A_{m}=-2.5 \times 10^{19} \mathrm{~cm}^{4} / \mathrm{s}$, and $K_{m}=0.3 \mathrm{~cm}^{2} / \mathrm{s}$. Taking the time mean of the above equation from step 1 to step $\mathrm{N}$ yields

$$
\frac{T^{N+1}-T^{1}}{N \cdot \Delta t}+\nabla \cdot(\overline{\vec{u}} \bar{T})+\frac{\partial(\bar{w} \bar{T})}{\partial z}=A_{m} \nabla^{4} \bar{T}+K_{m} \frac{\partial^{2} \bar{T}}{\partial z^{2}}+\nabla \cdot\left(-\overline{u^{\prime} T^{\prime}}\right)+\frac{\partial\left(-\overline{w^{\prime} T^{\prime}}\right)}{\partial z}
$$

The term $\frac{T^{N+1}-T^{1}}{N \cdot \Delta t}$ vanishes as $N \Delta t$ goes to infinity, and the above equation is simplified to the steady state conservation equation for the time mean tracer concentrations. The two extra terms on the RHS (right hand side) are the fluxes caused by time-dependent processes.

For the five-year, 600-sample means, the individual terms in eq. (4.13) can be directly computed and some of them are shown in Fig. 4.4. The numbers at the bottom of the panels are the contour intervals (the number in the middle), the minimum values (the numbers on the left) and the maximum values (the numbers on the right) for the plots above these numbers. The contours start from zero values (the first solid line adjacent to the dashed lines), increase with the contour intervals towards the maximum values, and decrease to the minimum values. 
It can be seen that these terms approximately satisfy eq.(4.13). The small imbalances are mainly caused by the subsampling in the time domain in computing the 5-year time means (The data were sampled at about 3 days time interval while the time step in the GCM was much smaller). More discussion on the aliasing effects of subsampling can be found in section 4.4, taking the space domain as an example.

The advection and horizontal eddy fluxes are dominant terms in the tracer conservation equations. Even when the means are taken over a 5-year time period, the temporal variation term $\frac{T^{N+1}-T^{1}}{N \cdot \Delta t}$ has importance in the tracer balances: their values have the same magnitudes as those of the vertical eddy and diffusion fluxes. For this fine grid resolution case, the biharmonic diffusion terms are also important (it will be shown in section 4.4 that this term is negligible in a larger grid spacing).

The tracer conservation equations used in the inverse model are in the form of

$$
\nabla \cdot(\overline{\vec{u}} \bar{T})+\frac{\partial(\bar{w} \bar{T})}{\partial z}=\nabla(A \nabla T)+\frac{\partial}{\partial z}\left(K \frac{\partial T}{\partial z}\right)
$$

where the two terms on the RHS were introduced with the intention to parameterize the eddy fluxes. Various terms in eq. (4.13) are not explicitly included in eq.(4.14), and their effects in the tracer balances will be picked up by the other terms in eq.(4.14) to some extent in the process of obtaining the inverse model solutions. This fact must be kept in mind in interpreting the inverse model results. 

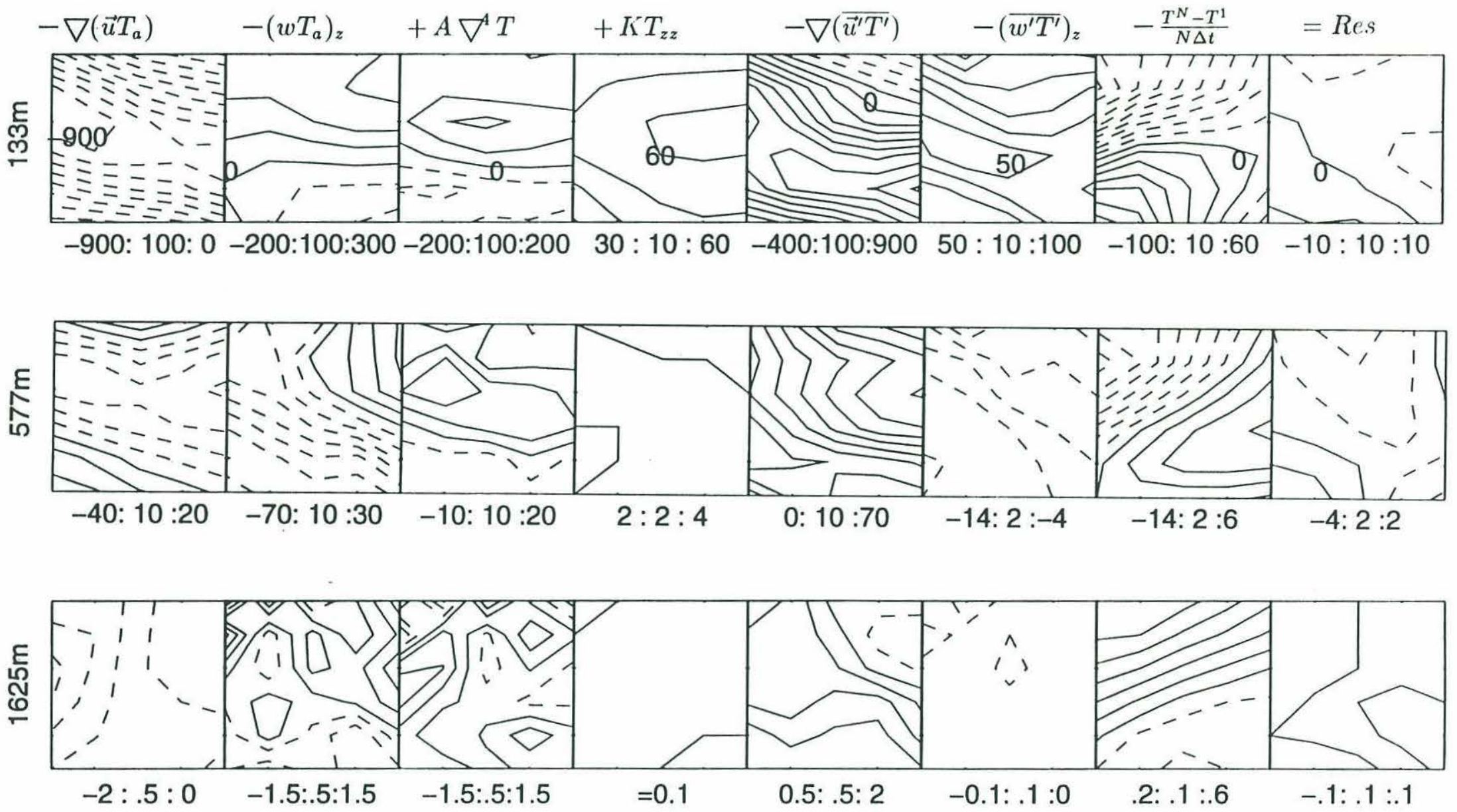

Fig. 4.4. Terms $\left(10^{-10}{ }^{\circ} \mathrm{C} / \mathrm{sec}\right)$ in the conservation equation for the 5-year time-mean temperature anomaly ( $T_{a}$, anomaly from the area-averaged value at the depth concerned) at three depths. Numbers at the bottoms are the contour values and intervals. 


\subsubsection{Inverse Model Results of the Time-Mean Fields}

The inverse model described in Chapter 2 and used in Chapter 3 is applied to the time mean fields of the EGCM ocean, with a grid resolution of $2 / 5^{\circ}$ longitude by $1 / 3^{\circ}$ latitude and by 13 levels in the vertical. The knowns (input) for the inverse model are the time mean potential temperature and salinity fields (as well as the time-mean density field). The unknowns of the inverse model are the horizontal circulations in terms of the streamfunctions, the vertical velocities, as well as the horizontal and vertical eddy diffusion coefficients which are intended to parameterize the eddy flux terms.

Based on the results of the parameterization experiments in Chapter 3, the parameters for velocities (the streamfunction and the vertical velocity) are treated as point-wise unknowns, while the horizontal and vertical "diffusion" coefficients A and $\mathrm{K}$ in the tracer conservation equations are parameterized as two-dimensional (in the horizontal plane), the third-order discrete Tchebychev polynomial functions whose coefficients vary from depth to depth.

\section{Horizontal Circulations}

The 5-year time-mean EGCM circulations in the inverse model domain are shown in Fig. 4.5 together with their statistical error ellipses. Note that the vector scales vary from depth to depth. Generally, the circulations are weaker and the errors are smaller as depth increases. The area-averaged velocities on the 13 levels are $3.37,2.55,1.71,0.97,0.48,0.36,0.42,0.34,0.21,0.15,0.19,0.26$, and $0.26 \mathrm{~cm} / \mathrm{s}$ respectively. At greater depth (say below $300 \mathrm{~m}$ ), the statistical errors are so large that the circulations are generally insignificantly different from zero. 
The horizontal circulations estimated by the inverse model from the time mean EGCM hydrographic data are shown in Fig. 4.6. The vector scales are the same as those used in Fig. 4.5. Comparisons of Fig. 4.6 with Fig. 4.5 show that the estimated circulations have the same flow patterns and magnitudes as those of the numerical GCM time means. Although there are some differences in detailed structures, these differences are generally insignificant within the statistical errors of the time means and inverse model estimated errors.

\section{Vertical Velocity}

The time-mean vertical velocities of the EGCM ocean at several depths are shown in Fig. 4.7, along with their statistical errors. In most of the region (except the top level at $111 \mathrm{~m}$ ), especially at greater depths, the statistical errors are so large that the values of the vertical velocity are indistinguishable from zero.

The vertical velocities estimated from the inversion are shown in Fig. 4.8 at the same depths. On the top surface at $111 \mathrm{~m}$, the estimated vertical velocity has the same sign as that of the GCM time means in most of the area. The differences in values are not significant except at the southwest corner, due to the large inverse model estimated errors and the GCM statistical errors. On a deeper level at 1500 $\mathrm{m}$, the inverse model estimated vertical velocities even has opposite signs as those of the GCM ones. But again, due to the even larger statistical errors, the differences between the inversion and GCM time means are generally insignificant. 
GCM Circuiations: $2 / 5 \times 1 / 3$ degrees resolution

$133.19 \mathrm{~m}$

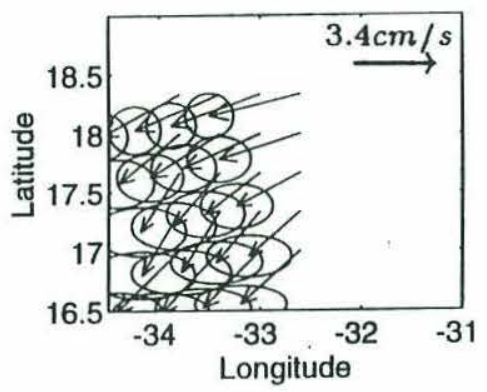

$295.025 m$

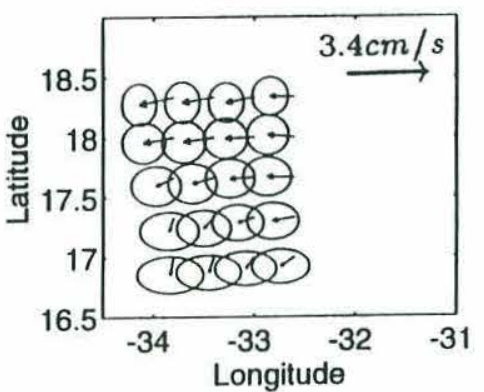

$577.365 \mathrm{~m}$

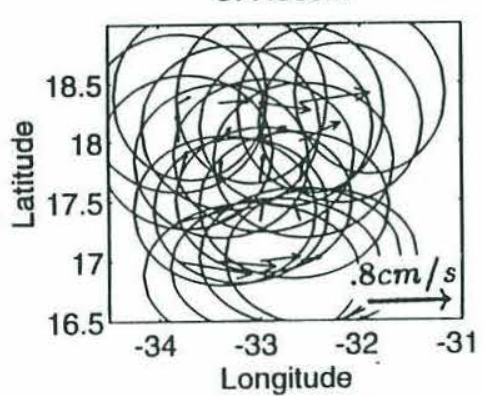

$179.52 \mathrm{~m}$
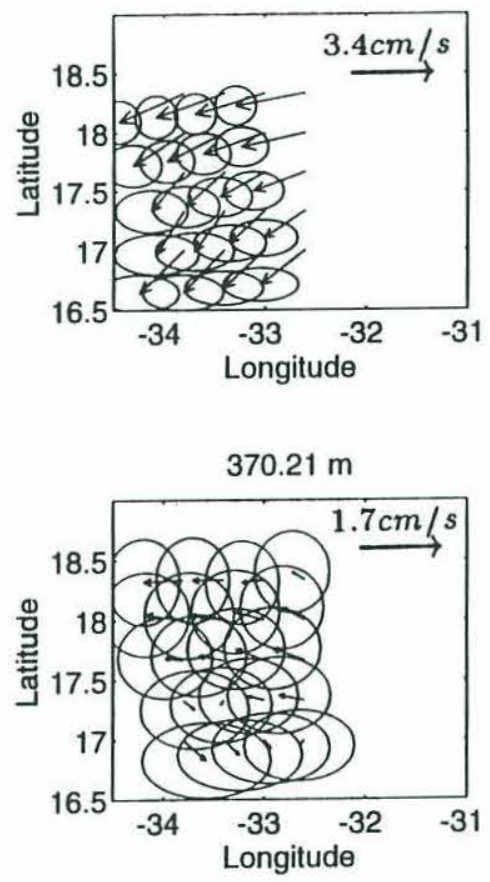

$721.465 \mathrm{~m}$

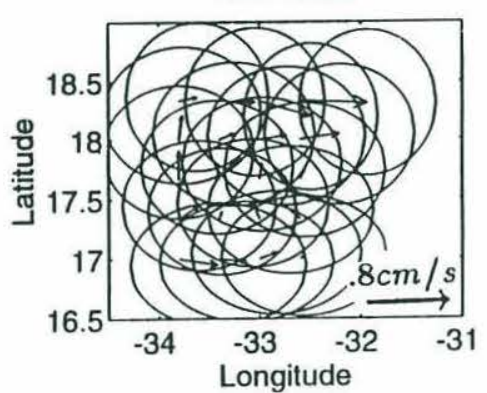

$232.595 m$
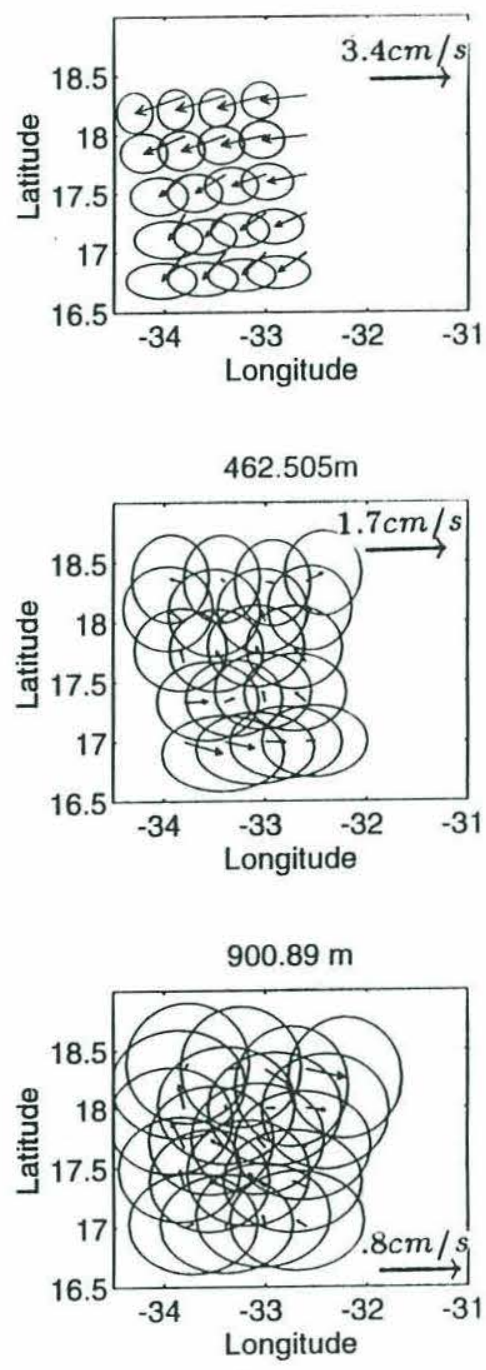

Fig. 4.5. Time-mean EGCM horizontal circulations with their statistical error ellipses. The arrows show the vector scales at difference depths. 

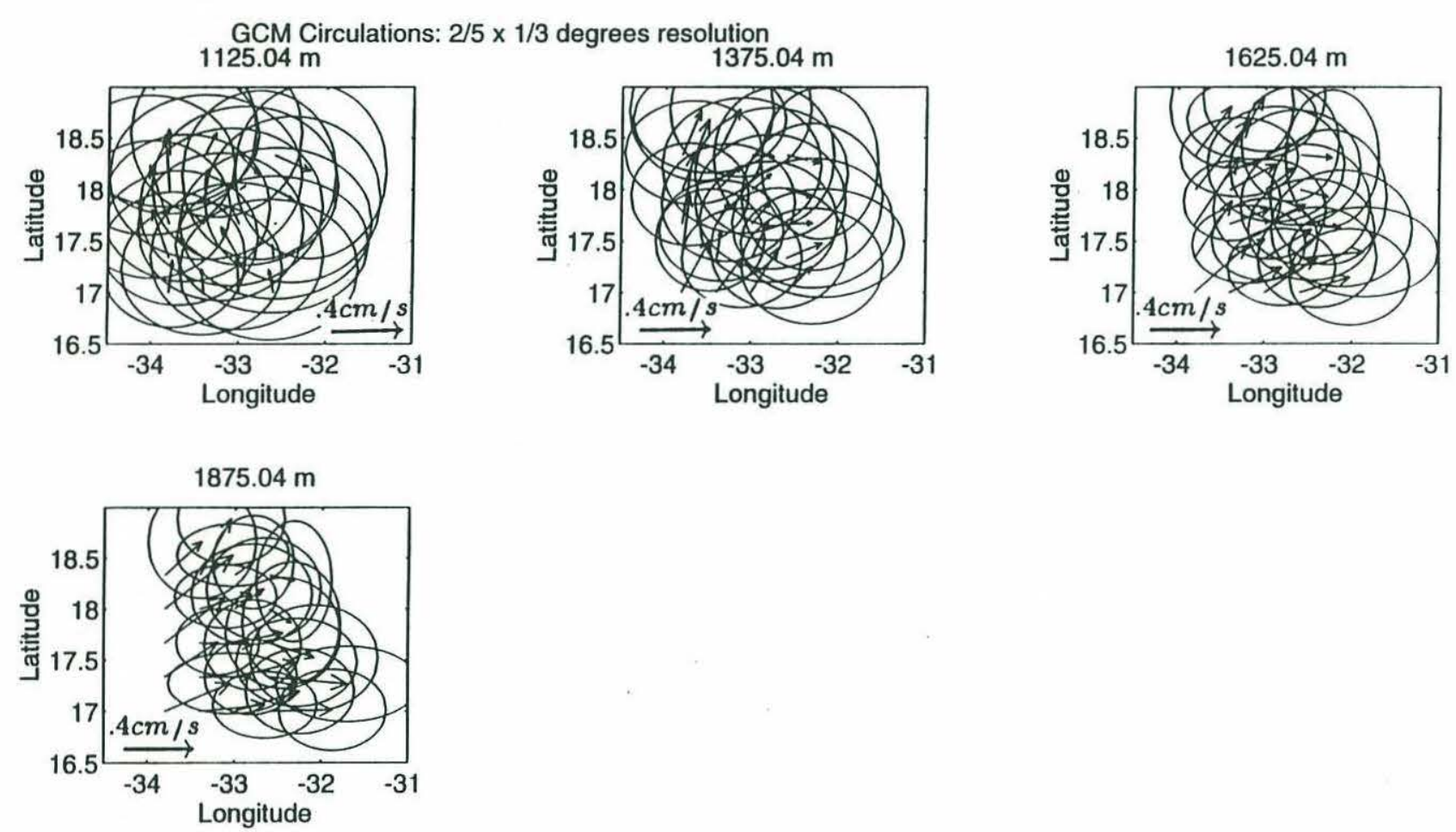

Fig. 4.5 (cont.) 
Estimated horizontal circulations with error ellipses. $2 / 5$ by $1 / 3$ degrees resolution

$133.19 \mathrm{~m}$

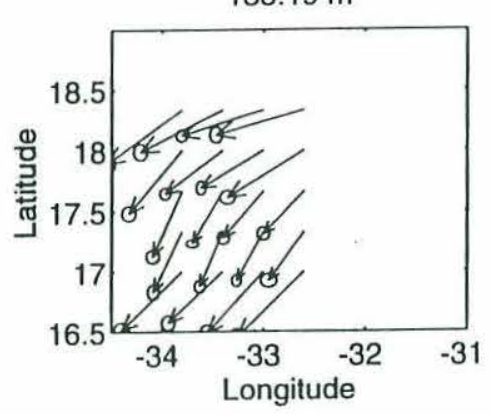

$295.025 m$
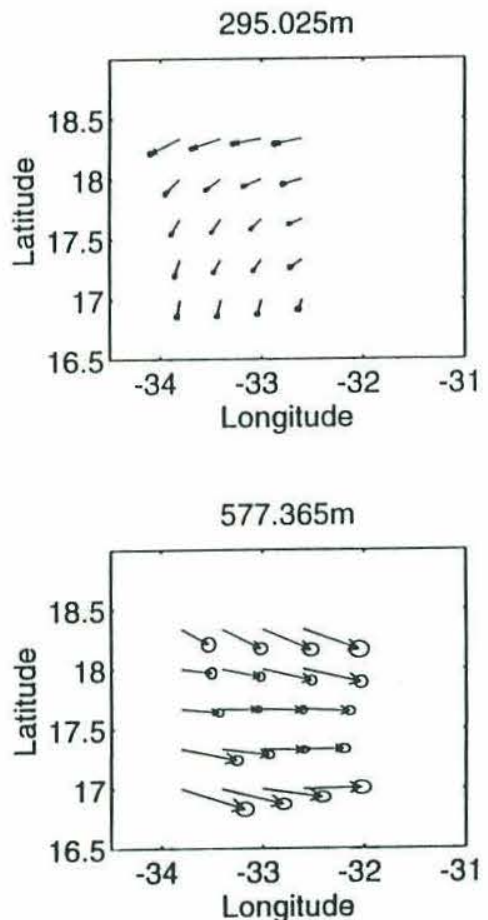

$179.52 \mathrm{~m}$

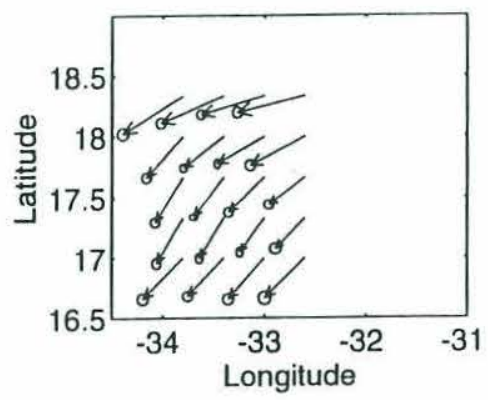

$370.21 \mathrm{~m}$

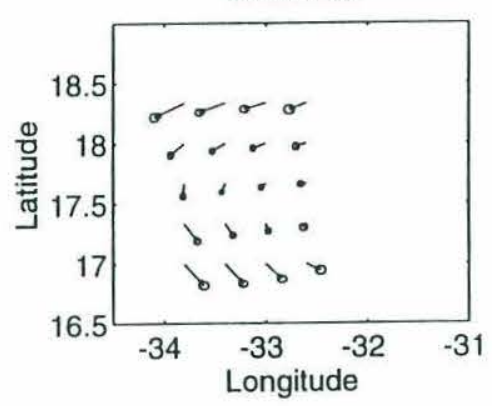

$721.465 m$

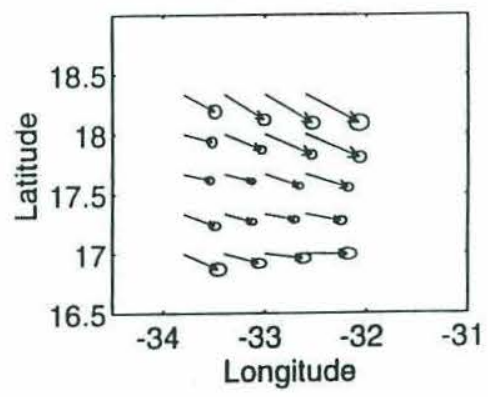

$232.595 \mathrm{~m}$
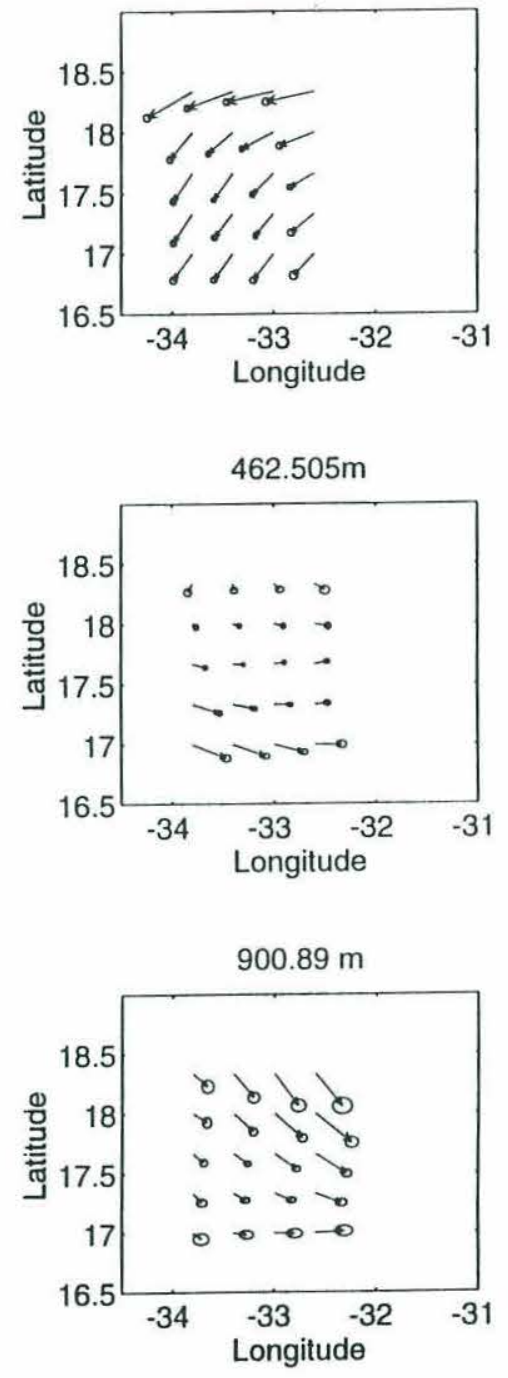

Fig. 4.6. Estimated horizontal circulations with their error ellipses from the time-mean EGCM hydrographic data in the fine grid resolution. Vector scales are the same as in Fig. 4.5. 
$1125.04 \mathrm{~m}$

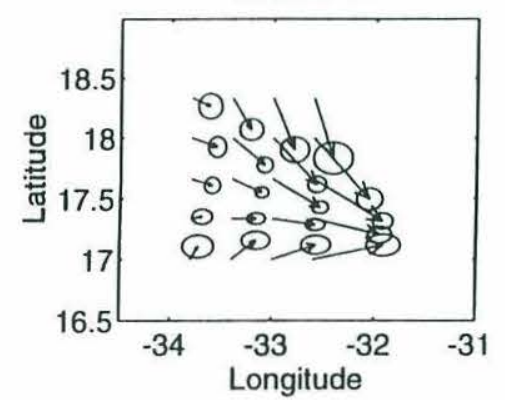

セ্

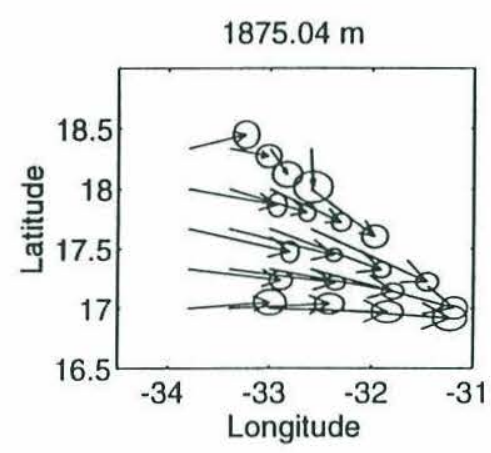

$1375.04 \mathrm{~m}$
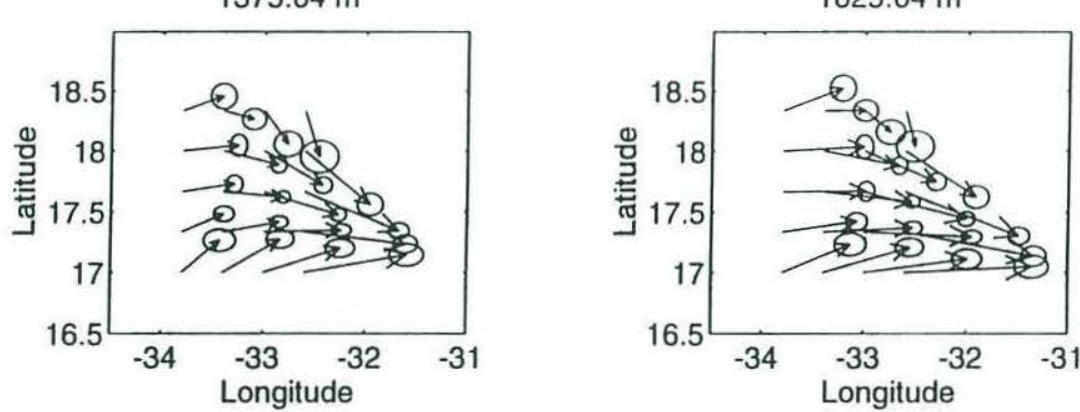

Fig. 4.6 (cont.) 

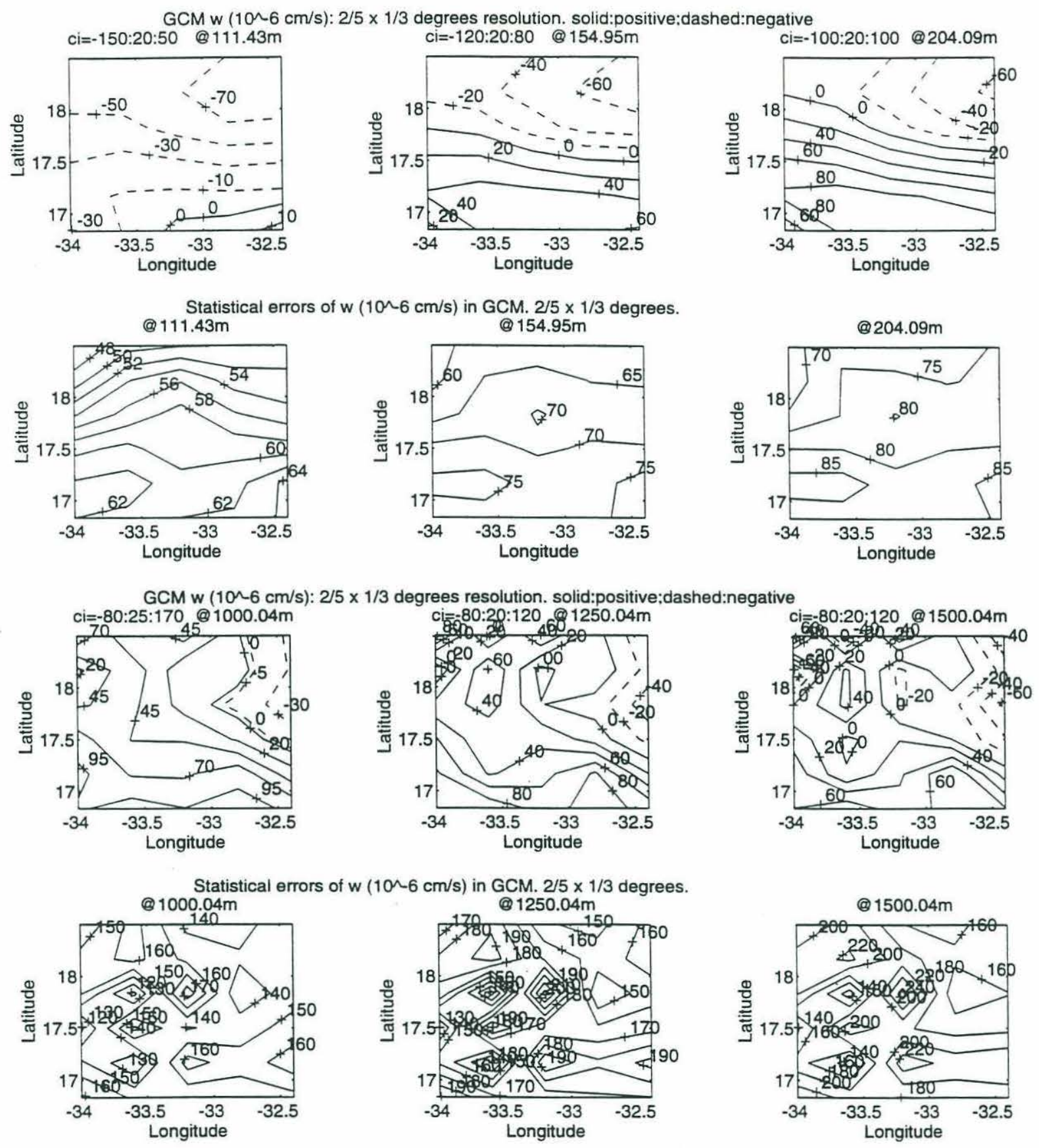

Fig. 4.7. Time-mean EGCM vertical velocities and their statistical errors (unit: $10^{-6} \mathrm{~cm} / \mathrm{s}$ ). 


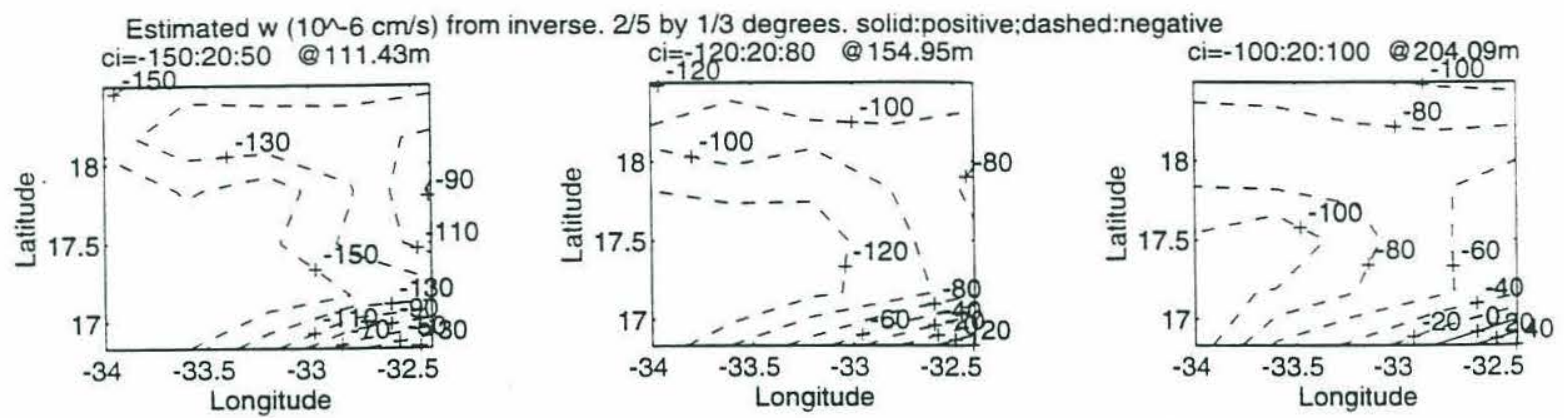

Estımated errors of $w$ ( $10 \sim 6 \mathrm{~cm} / \mathrm{s}$ ) trom inverse. $2 / 5$ by $1 / 3$ degrees resolution.
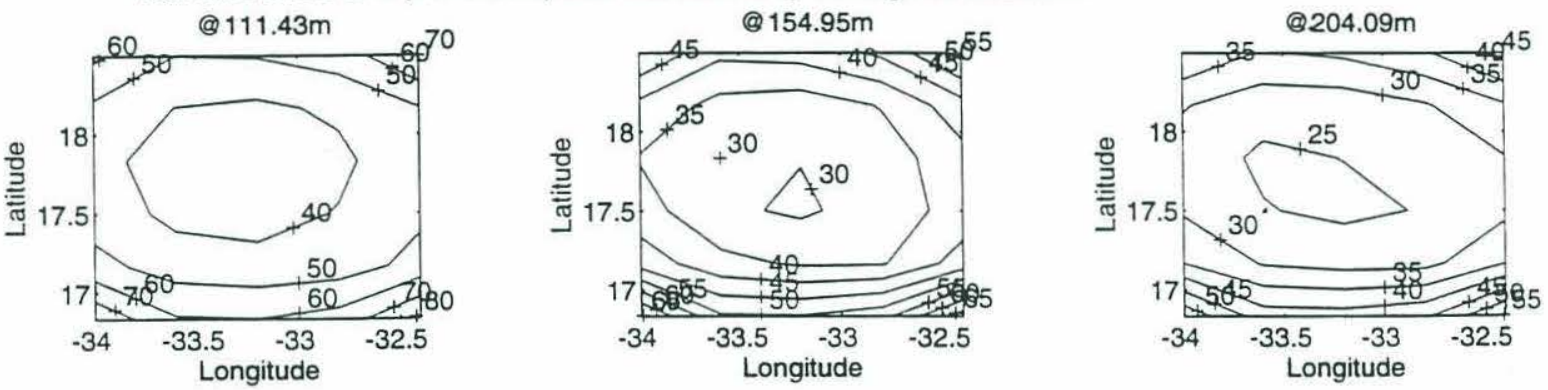

Estimated $w(10 \Omega-6 \mathrm{~cm} / \mathrm{s}$ ) from inverse. $2 / 5$ by $1 / 3$ degrees. solid:positive;dashed:negative
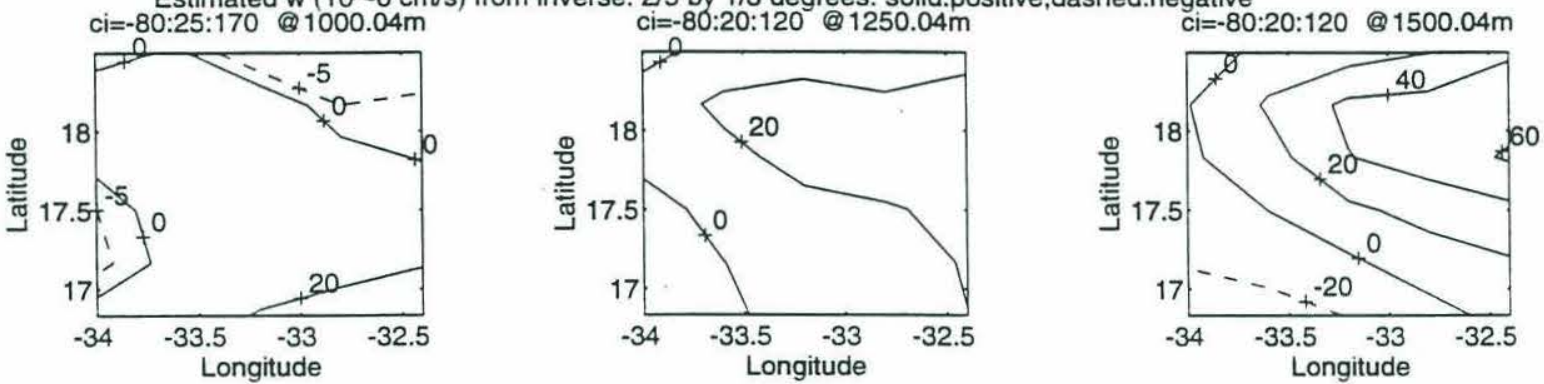

Estimated errors of $w(10 \Omega 6 \mathrm{~cm} / \mathrm{s})$ from inverse. $2 / 5$ by $1 / 3$ degrees resolution.
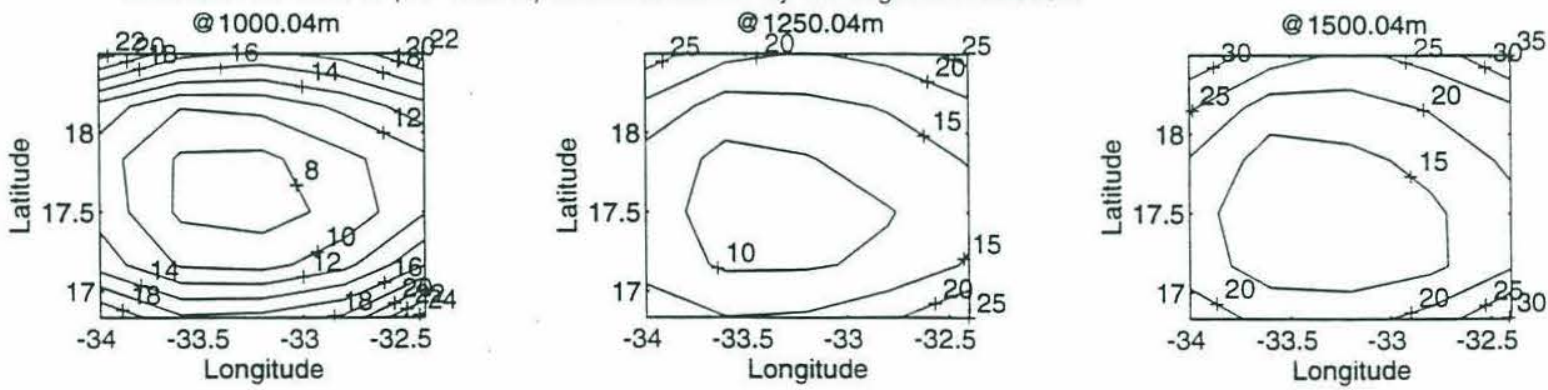

Fig. 4.8. Same as Fig. 4.6 but for vertical velocities $\left(10^{-6} \mathrm{~cm} / \mathrm{s}\right)$. 
In terms of closeness to the "data" values (regardless of the errors for the moment), the inverse model solutions for the vertical velocity are not as good as those for the horizontal circulations. The reason is that first, the solutions for the vertical velocity are only constrained by the conservation equations for heat and salt as well as the continuity equation, while the solutions for the horizontal circulation are further constrained by the dynamic equations (the thermal wind relation). As discussed in section 4.3.2, the continuity equation and thermal wind relation are quite accurate (errors are generally less than 5\%) in the time-mean fields of the EGCM ocean, while the steady inverse model conservation equations are not satisfied by the time-mean fields of the numerical GCM ocean (e.g., the neglected temporal variation is obviously an important term).

Secondly, the relatively "poor" estimates for the vertical velocity is related to the deviations of the estimates from their "true" values in the horizontal velocities. Although the inverse model estimated horizontal circulations are statistically consistent with those of the EGCM time means, the values of the horizontal velocities are not the same. Small deviations in the horizontal velocities, although they might not be significant compared to the velocities themselves, can result in large changes in the divergence of the horizontal velocity, thus produce significant changes in the values of the vertical velocity through the continuity equation.

Thirdly, additional biases of the estimated vertical velocity from the numerical GCM time means can be introduced by the neglected but yet significantly important terms in the conservation equations for tracers (e.g., the temporal variation term, the biharmonic dissipation terms). Fig. 4.3 shows that between $900 \mathrm{~m}$ and $1625 \mathrm{~m}$, the temporal variation term and the biharmonic dissipation term have 
larger magnitudes than the vertical advection term, and they have more organized patterns.

\section{Eddy Diffusion Coefficients}

It has long been recognized that eddy activities play an important role in the transport of water properties. However, computations of eddy fluxes are far from simple due to insufficient time and spatial resolutions of the ocean data. $\mathrm{Pa}$ rameterizing the eddy fluxes, or finding relations between the eddy fluxes and the large-scale time-mean fields, has been and will remain to be a difficult problem in oceanography (for a review, see Holloway, 1989).

As pointed out at the end of section 4.3.2, in the inverse model the eddy fluxes are parameterized as Fickian diffusion and the diffusivity tensor is assumed to be diagonal and isotropic but inhomogeneous in the geopotential coordinate. This is a highly simplified parameterization, although it has been widely used in the modeling community. In this parameterization it is assumed that the eddy transfer is parallel to the mean gradient. Non-gradient-parallel eddy transfer, or "skew flux" in some literature, in atmospheres was discussed by Green (1970), Stone (1972), Wallace (1978), and Plumb (1979). In the oceanographic context, related ideas are seen in Haidvogel and Rhines (1983), Middleton and Garrett (1986), and Middleton and Loder (1989). In Redi's (1982) paper, it was shown that when a diagonal second-rank diffusivity tensor in the isopycnal coordinate system is transformed to the geopotential coordinate system, the diffusivity tensor contains off-diagonal elements. More difficult, the relative size of the off-diagonal terms (skew fluxes) compared to the diagonal terms (parallel gradient fluxes) are unknown. 
What is physically important are the eddy fluxes, not the eddy diffusivity coefficients themselves. If there is a way to estimate the eddy fluxes directly, there would be no need to be troubled with computing the eddy diffusivity coefficients. In the conservation equations for $T$ and $S$ in the inverse model, if the eddy fluxes $-\overline{u^{\prime} T^{\prime}},-\overline{v^{\prime} T^{\prime}},-\overline{w^{\prime} T^{\prime}},-\overline{u^{\prime} S^{\prime}},-\overline{v^{\prime} S^{\prime}},-\overline{w^{\prime} S^{\prime}}$ were kept as individual unknowns, there would be too many unknowns to have them well resolved (the equation system is apparently underdetermined). On the other hand, if the eddy fluxes are parameterized as the diffusion coefficients $A$ and $K$ as in eq.(4.14), the 6 unknowns are reduced to 2 at every grid points. In dealing with this complicated and unsolved issue, we choose to use the Fickian diffusion with a second-rank diffusivity tensor in the geopotential coordinate system. With the available data in this study, it is difficult to estimate the accuracy of this assumption. For example, in

$$
-\overline{u^{\prime} T^{\prime}}=K_{x x} \frac{\partial \bar{T}}{\partial x}+K_{x y} \frac{\partial \bar{T}}{\partial y}+K_{x z} \frac{\partial \bar{T}}{\partial z}
$$

it is difficult to estimate how large the off-diagonal terms, $K_{x y} \frac{\partial \bar{T}}{\partial y}$ and $K_{x z} \frac{\partial \bar{T}}{\partial z}$, are compared to the diagonal term $K_{x x} \frac{\partial \bar{T}}{\partial x}$. But at least there are two issues we can check: given that the diffusivity tensor is diagonal, is it horizontally isotropic? Is it the same for $T$ and $S$ ? The following paragraph answers the two questions with direct computations.

i) Eddy diffusion coefficients directly calculated from the eddy fluxes of the numerical GCM ocean 
From the available time series of the numerical GCM ocean, the eddy heat and salt fluxes $-\overline{u^{\prime} T^{\prime}},-\overline{v^{\prime} T^{\prime}},-\overline{w^{\prime} T^{\prime}},-\overline{u^{\prime} S^{\prime}}, \overline{v^{\prime} S^{\prime}},-\overline{w^{\prime} S^{\prime}}$ can be computed as

$$
\overline{u^{\prime} T^{\prime}}=\frac{1}{N} \sum_{n=1}^{N} u_{n}^{\prime} T_{n}^{\prime}=\frac{1}{N} \sum_{n=1}^{N}\left(u_{n}-\bar{u}\right)\left(T_{n}-\bar{T}\right)
$$

where $\mathrm{N}=600, \bar{u}$ and $\bar{T}$ are the time means. The $u^{\prime} T^{\prime}$ and $v^{\prime} T^{\prime}$ are computed at the same points as the $u T$ and $v T$ in the GCM, i.e. at the four boundaries of the finite difference boxes. The variance of the eddy fluxes are estimated as

$$
\operatorname{var}\left(\overline{u^{\prime} T^{\prime}}\right)=\frac{1}{N} \sum_{n=1}^{N}\left(u_{n}^{\prime} T_{n}^{\prime}-\overline{u^{\prime} T^{\prime}}\right)^{2}=\frac{1}{N} \sum_{n=1}^{N}\left(u_{n}^{\prime} T_{n}^{\prime}\right)^{2}-\left(\overline{u^{\prime} T^{\prime}}\right)^{2}
$$

Using the integral time scales computed in section 4.2 (Table 4.1), the statistical errors of the time-mean eddy fluxes can be calculated as

$$
\left(\overline{u^{\prime} T^{\prime}}\right)_{e}=\left[\operatorname{var}\left(\overline{u^{\prime} T^{\prime}}\right) / d f\right]^{1 / 2}=\left[\operatorname{var}\left(\overline{u^{\prime} T^{\prime}}\right) /(P / 2 \Gamma)\right]^{1 / 2}
$$

where $\mathrm{P}=5$ years and $\Gamma$ is the integral time scale shown in Table 4.1.

Using the assumption of Fickian diffusion with a second-rank diffusion tensor, the eddy fluxes computed above can be used to compute the eddy diffusion coefficients:

$$
A_{x}^{T}=-\overline{u^{\prime} T^{\prime}} / \frac{\partial \bar{T}}{\partial x} ; \quad A_{y}^{T}=-\overline{v^{\prime} T^{\prime}} / \frac{\partial \bar{T}}{\partial y} ; \quad A_{z}^{T}=-\overline{w^{\prime} T^{\prime}} / \frac{\partial \bar{T}}{\partial z} .
$$

Similar diffusion coefficients can be defined for the eddy salt fluxes. Theoretically, in estimating the errors of the diffusion coefficients computed above, both the error of 
$-\overline{u^{\prime} T^{\prime}}$ and the error of $\frac{\partial \bar{T}}{\partial x}$ should be taken into account. As the statistical errors of the time-mean tracers are very small (c.f. section 4.2), their effects can be neglected and thus the errors can be estimated as (except where $\frac{\partial \bar{T}}{\partial x}$ is close to zero)

$$
\left(A_{x}^{T}\right)_{e}=\left(-\overline{u^{\prime} T^{\prime}}\right)_{e} / a b s\left(\frac{\partial \bar{T}}{\partial x}\right)
$$

These estimates can be regarded as lower bounds for the errors of the eddy diffusion coefficients.

a) Horizontal Eddy Diffusion Coefficients:

are they isotropic and are they the same for heat and salt?

Shown in Fig. 4.9 are examples of the horizontal eddy diffusion coefficients and their statistical errors at several depths directly computed from the GCM eddy fluxes. It can be seen that within the statistical errors, the eddy diffusion coefficients are only marginally significant.

Comparison of the values of $A_{x}^{T}$ (Fig. 4.9a) with those of $A_{y}^{T}$ (Fig. 4.9b) shows that the horizontal eddy diffusion coefficients might be anisotropic. For example, at $233 \mathrm{~m}$, the patterns of $A_{x}^{T}$ and those of $A_{y}^{T}$ are obviously different: where $A_{x}^{T}$ has minimum (negative) values $\left(\sim-1 \times 10^{7} \mathrm{~cm}^{2} / \mathrm{s}\right.$ in the central area), $A_{y}^{T}$ has maximum (positive) values $\left(\sim 8 \times 10^{7} \mathrm{~cm}^{2} / \mathrm{s}\right)$, and while $A_{x}^{T}$ increases south- and north-ward from the center, $A_{y}^{T}$ decreases. However, these numerical differences must be related to the statistics. Fig. 4.9 shows that with the $95 \%$ confidence intervals, the eddy diffusion coefficients themselves are barely significantly different from zero. Therefore, statistically it is hard to distinguish the differences between 
the values of the zonal and meridional eddy diffusion coefficients in most of the area, and thus the anisotropy is of no significance.

Comparison of $A_{y}^{T}$ (Fig. 4.9b) with $A_{y}^{S}$ (Fig. 4.9c) shows that they have very similar distribution patterns. This is also true for the eddy diffusion coefficients in the zonal direction (not shown). There are some differences in numerical values, but these differences are generally insignificant within the statistical errors.

In summary, in this numerical GCM ocean, the statistics shows that the anisotropy of the horizontal eddy diffusion coefficients are generally insignificant, and the horizontal eddy diffusion coefficients for heat and salt are about the same in most of the domain concerned.

b). Vertical Eddy Diffusion Coefficients

Some examples of the vertical eddy diffusion coefficients directly calculated from the EGCM eddy heat and salt fluxes are shown in Fig. 4.10, along with their statistical errors. Although the errors are also large, the values of the vertical diffusion coefficients are significantly above the errors at some depths. For instance, at $204 \mathrm{~m}$, the directly computed vertical eddy diffusion coefficients are significantly negative. Note that in the EGCM ocean, in addition to the eddy fluxes, there is also a vertical diffusion flux with a specified constant diffusion coefficient of $0.3 \mathrm{~cm}^{2} / \mathrm{s}$ (horizontally there is a biharmonic diffusion). By adding this number to the above eddy diffusion coefficients, the "total" diffusion coefficients are not significantly negative. 
In most of the water column, the values of $A_{z}^{T}$ and $A_{z}^{S}$ have similar spatial distribution patterns. The only exception is between the depths of $1000 \mathrm{~m}$ and 1500 m. The magnitudes of $A_{z}^{T}$ and $A_{z}^{S}$ are in the same order except at the deepest two depths $(1500 \mathrm{~m}$ and $1750 \mathrm{~m})$, where $a b s\left(A_{z}^{S}\right) \gg a b s\left(A_{z}^{T}\right)$. In terms of the numerical values themselves, $A_{z}^{S}$ is generally larger than $A_{z}^{T}$. In Fig. 4.10a and Fig. 4.10b, at $204 \mathrm{~m}$, although $a b s\left(A_{z}^{S}\right)>a b s\left(A_{z}^{T}\right)$, the differences are insignificant within the statistical errors. However, at $1000 \mathrm{~m}$, the value differences become significantly above the statistical errors.

The vertical eddy fluxes possess opposite sign of the vertical dissipation terms $\left(K_{m} T_{z z}\right)$ in the heat and salt conservation equations (see Fig. 4.3), and the vertical eddy diffusion coefficients have mostly opposite sign to $K_{m}$ and are mostly negative in the domain concerned. 

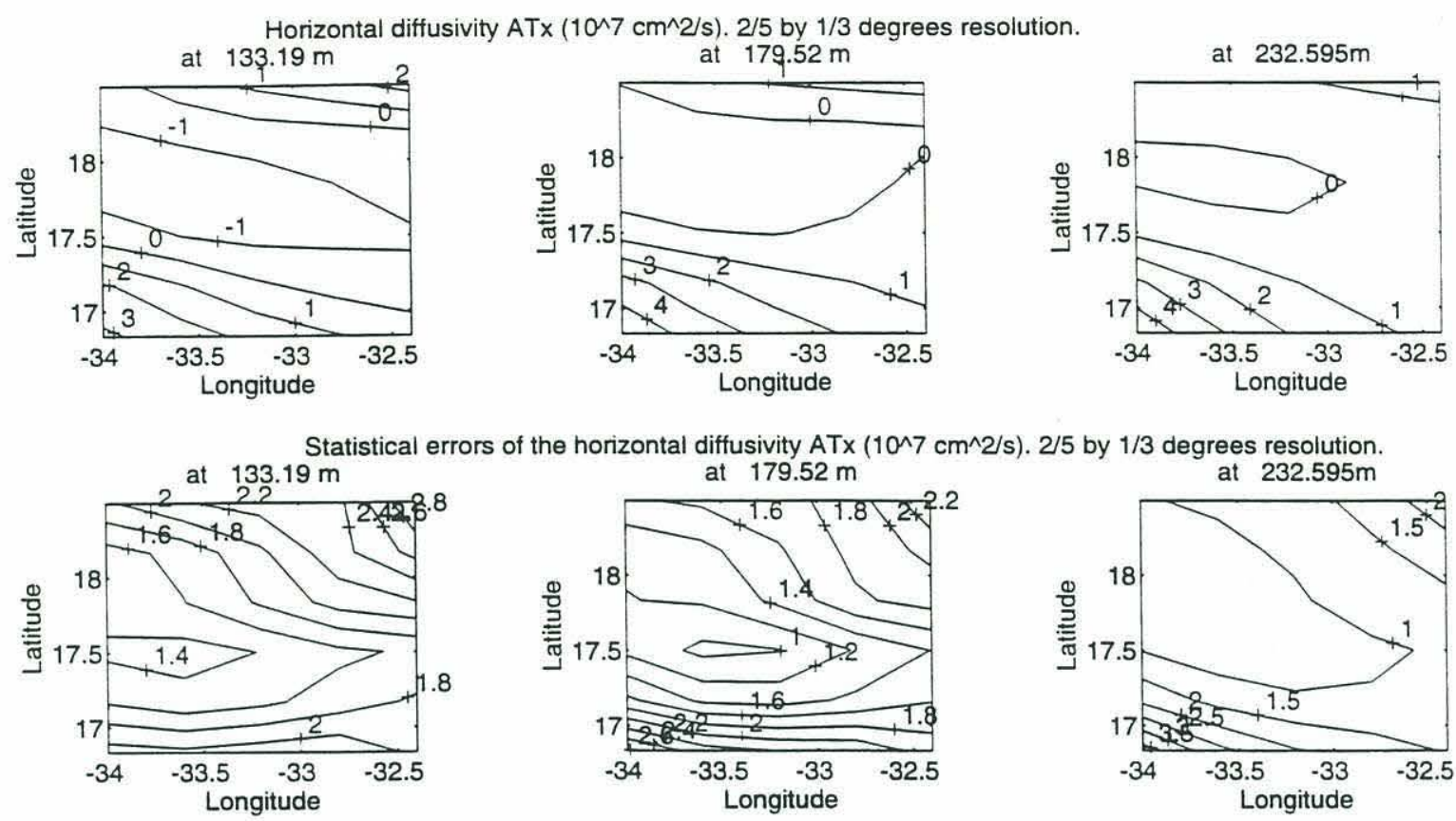

Horizontal diffusivity $\operatorname{ATx}\left(10^{\wedge} 7 \mathrm{~cm}^{\wedge} 2 / \mathrm{s}\right) .2 / 5$ by $1 / 3$ degrees resolution.
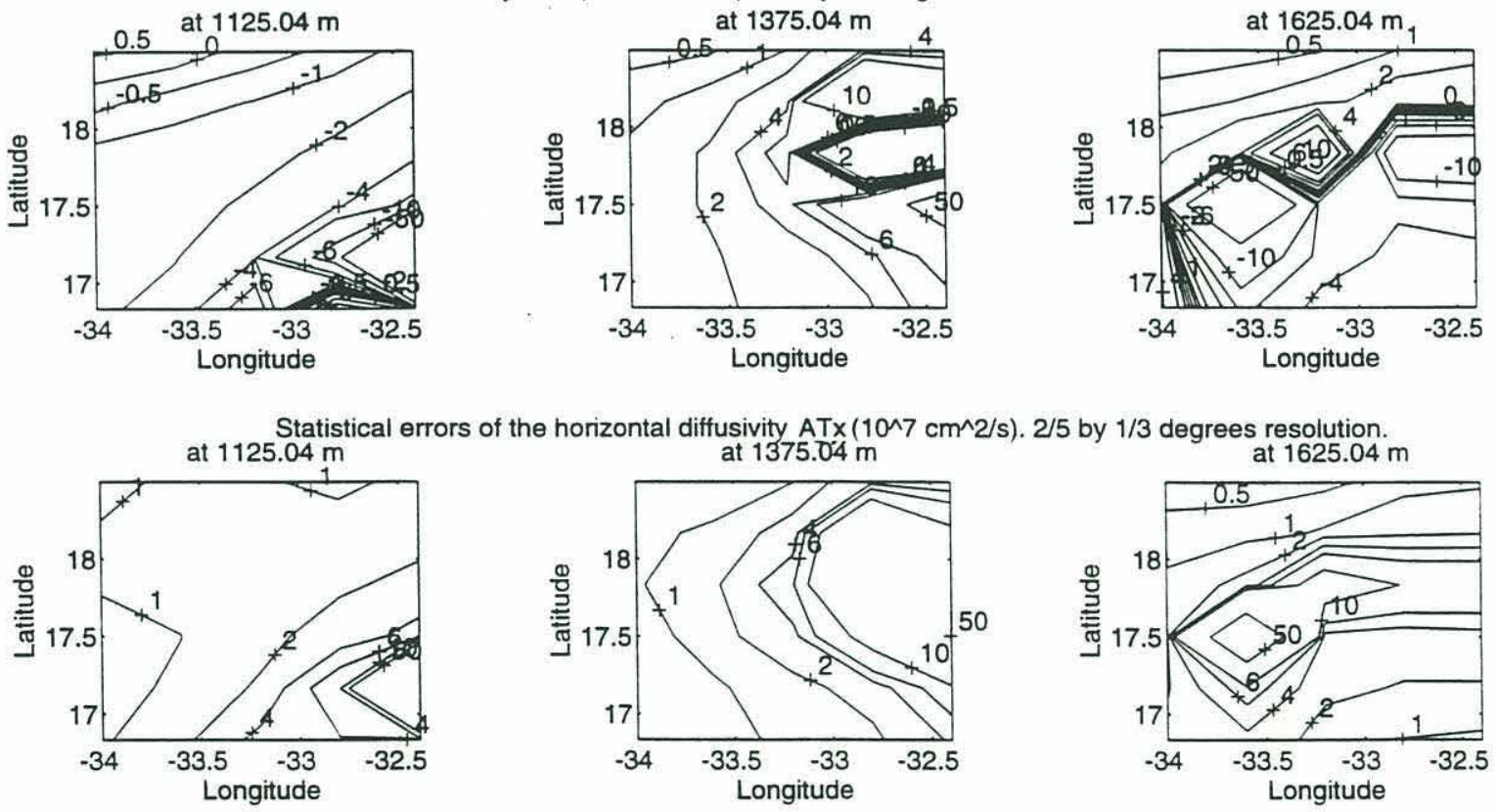

Fig. 4.9. Horizontal eddy diffusion coefficients and their statistical errors $\left(10^{7} \mathrm{~cm}^{2} / \mathrm{s}\right)$ computed from the EGCM eddy fluxes. (a) for heat fluxes in zonal direction; (b) for heat fluxes in meridional direction; (c) for salt fluxes in meridional direction. 
Horizontal diffusivity ATy $\left(10^{\wedge} 7 \mathrm{~cm}^{\wedge} 2 / \mathrm{s}\right) .2 / 5$ by $1 / 3$ degrees resolution.

at $133.19 \mathrm{~m}$

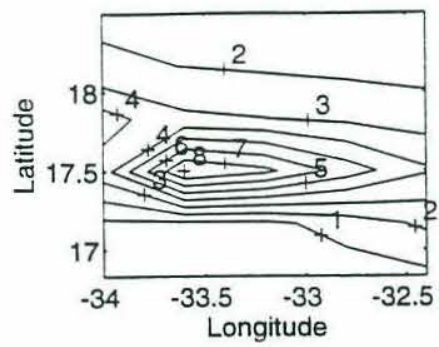

at $179.52 \mathrm{~m}$

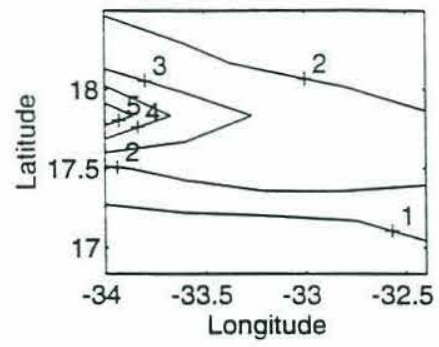

at $232.595 \mathrm{~m}$

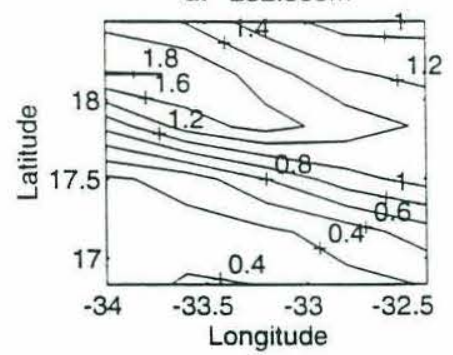

Statistical errors of the horizontal diffusivity ATy $\left(10 \wedge 7 \mathrm{~cm}^{\wedge} 2 / \mathrm{s}\right) .2 / 5$ by $1 / 3$ degrees resolution. at $133.19 \mathrm{~m}$

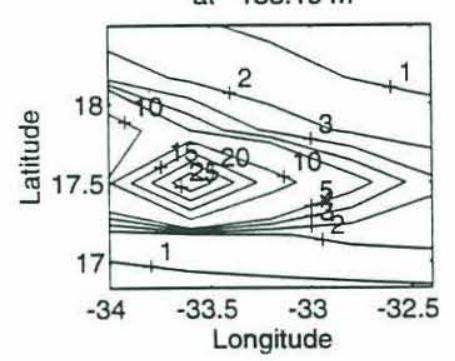
at $179.52 \mathrm{~m}$
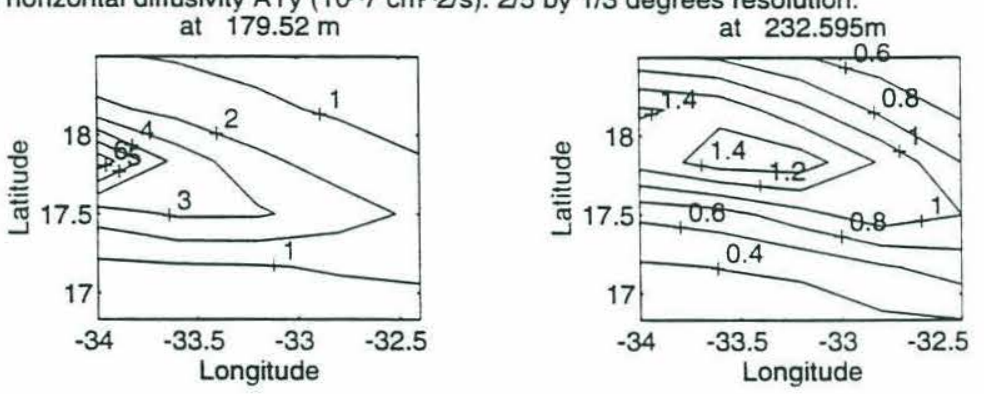

Horizontal diffusivity ATy ( $\left.10 \wedge 7 \mathrm{~cm}^{\wedge} 2 / \mathrm{s}\right) .2 / 5$ by $1 / 3$ degrees resolution.

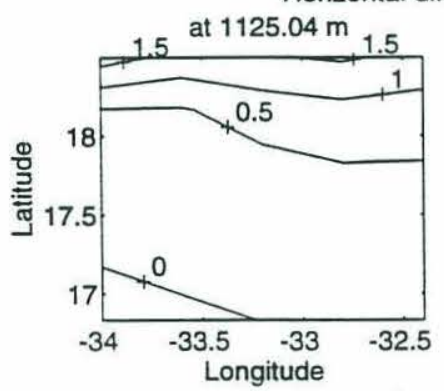

at $1375.04 \mathrm{~m}$
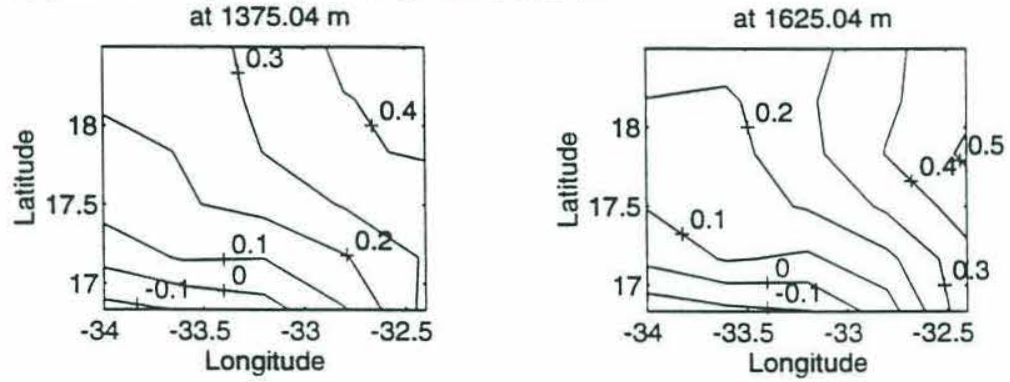

Statistical errors of the horizontal diffusivity ATy ( $\left.10 \wedge 7 \mathrm{~cm}^{\wedge} 2 / \mathrm{s}\right) .2 / 5$ by $1 / 3$ degrees resolution.
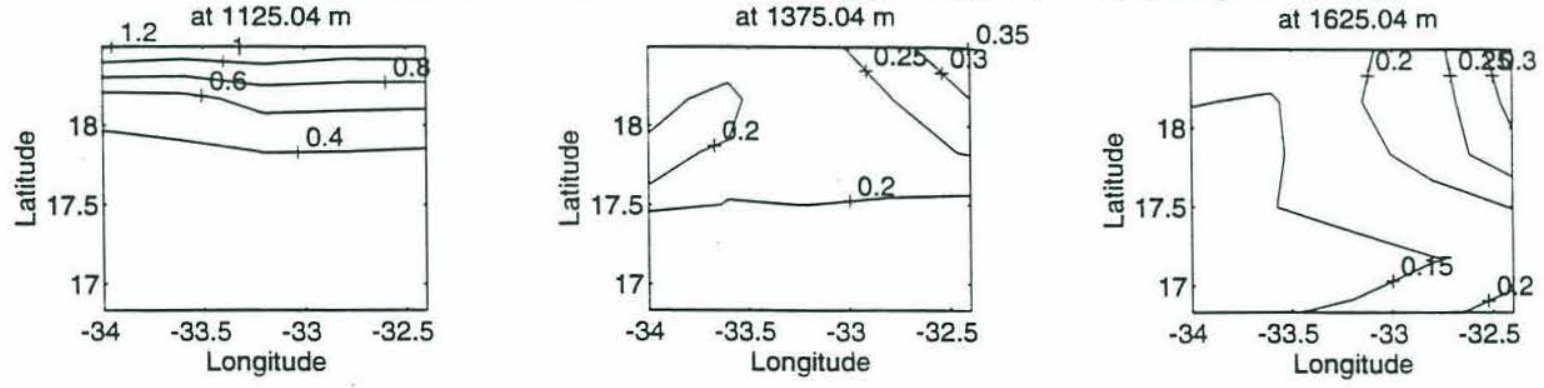

Fig. 4.9 (b) 

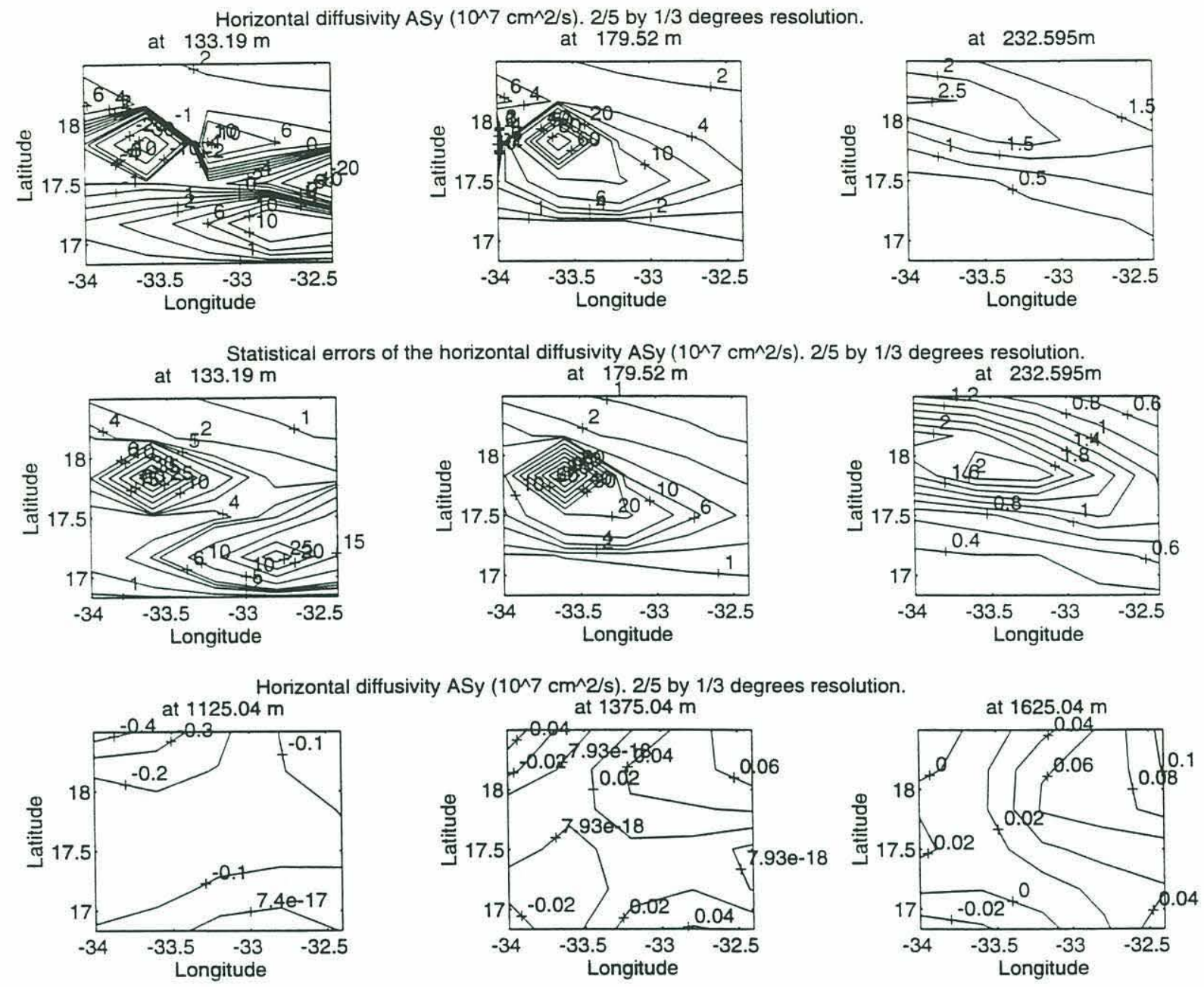

Statistical errors of the horizontal diffusivity ASy $\left(10^{\wedge} 7 \mathrm{~cm}^{\wedge} 2 / \mathrm{s}\right) .2 / 5$ by $1 / 3$ degrees resolution.
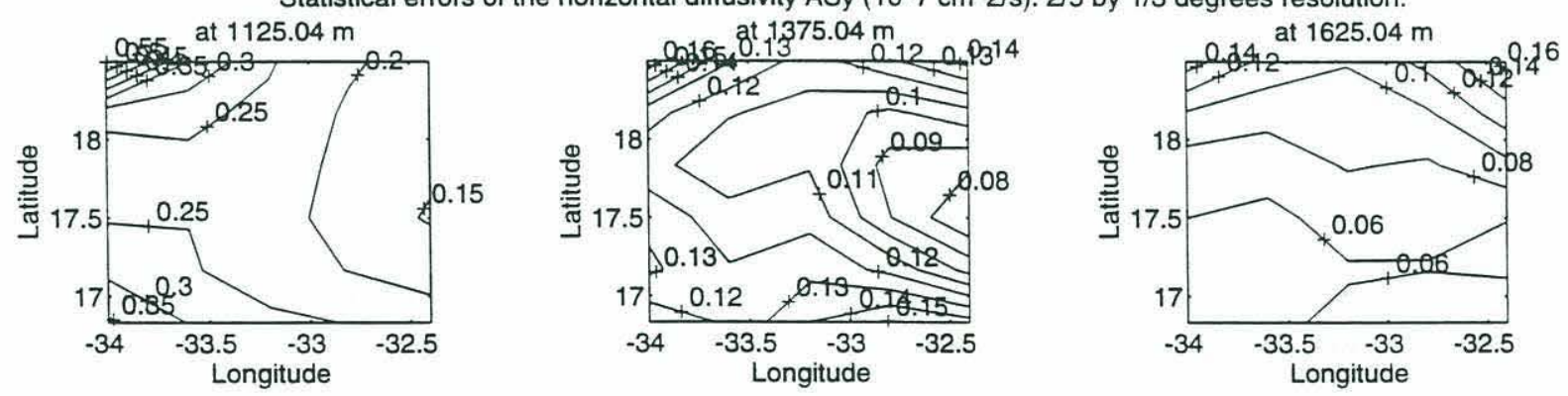

Fig. 4.9 (c) 

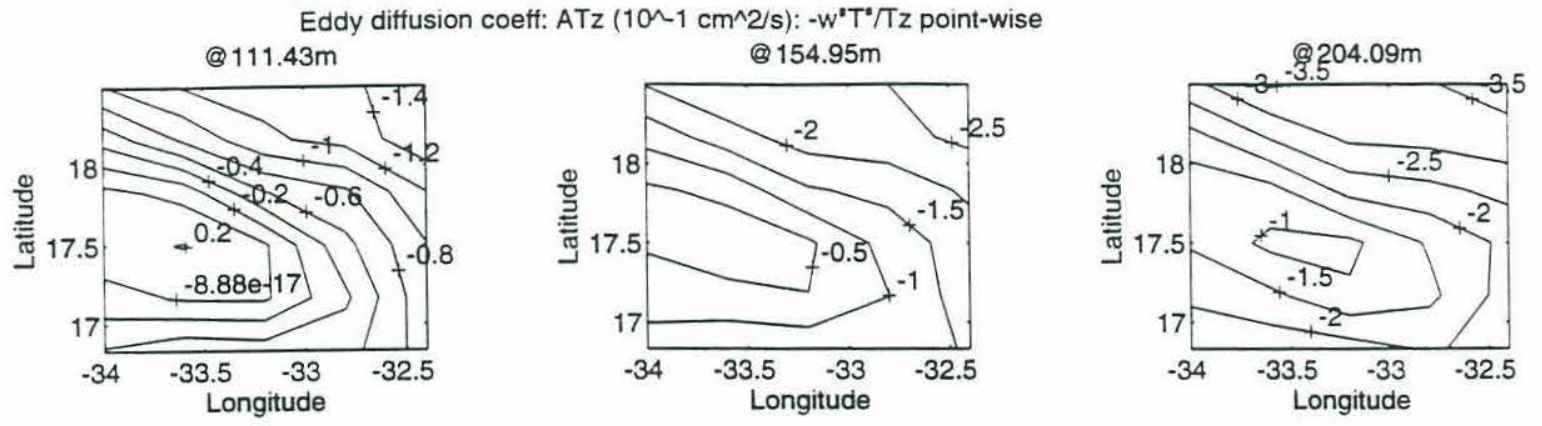

Statistical Errors of ATz (10^ $\left.1 \mathrm{~cm}^{\wedge} 2 / \mathrm{s}\right):-\mathrm{W} T \mathcal{T} \mathrm{T} z$ point-wise
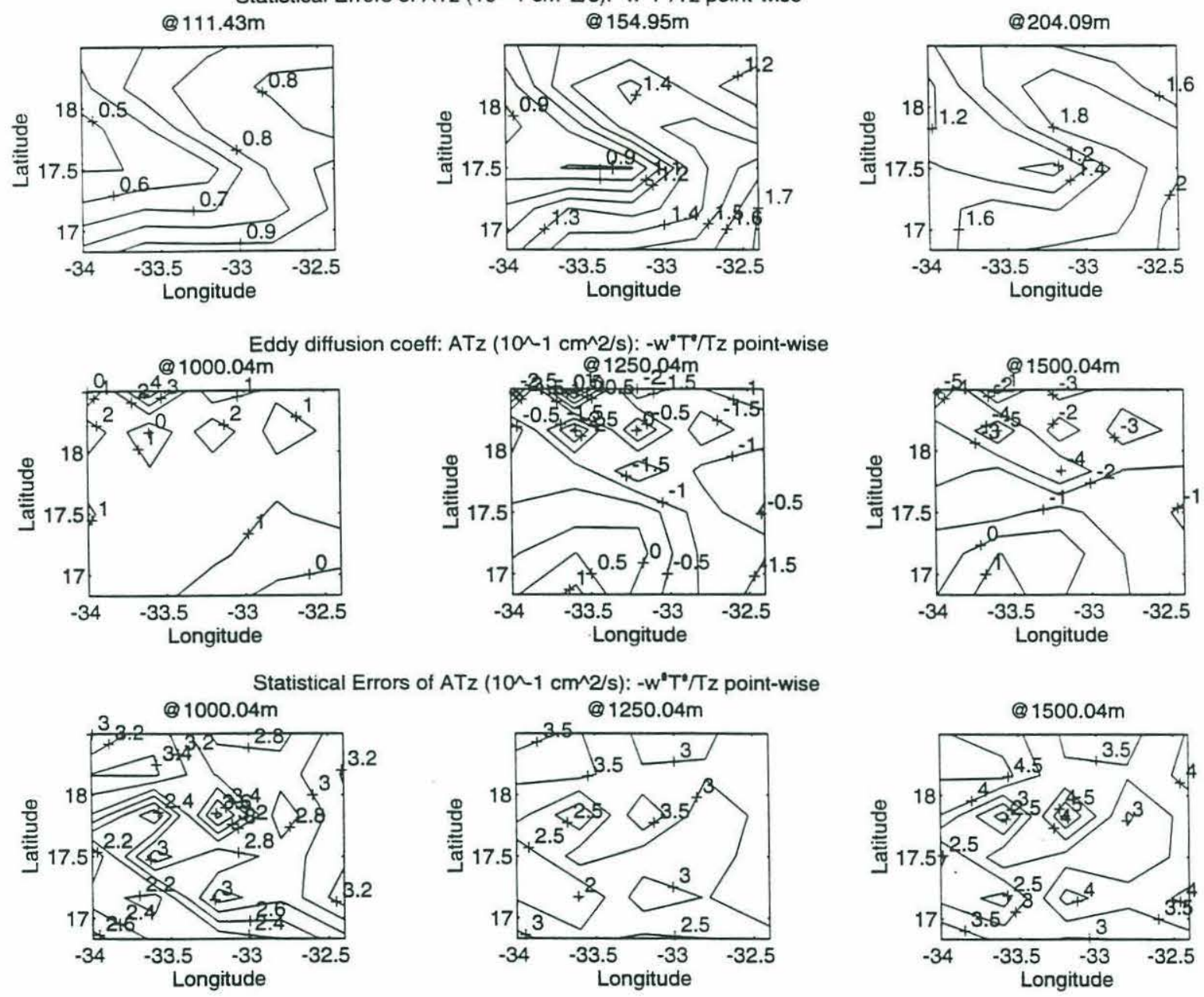

Fig. 4.10. Vertical eddy diffusion coefficients and their statistical errors $\left(\mathrm{cm}^{2} / \mathrm{s}\right)$ computed from the EGCM eddy fluxes. (a) for heat fluxes; (b) for salt fluxes. 
Eddy diffusion coeff: $\mathrm{ASz}$ ( $\left.10^{\wedge} 1 \mathrm{~cm} \wedge 2 / \mathrm{s}\right)$ : $-\mathrm{w}^{*} \mathrm{~S}^{\bullet} / \mathrm{Sz}$ point-wise
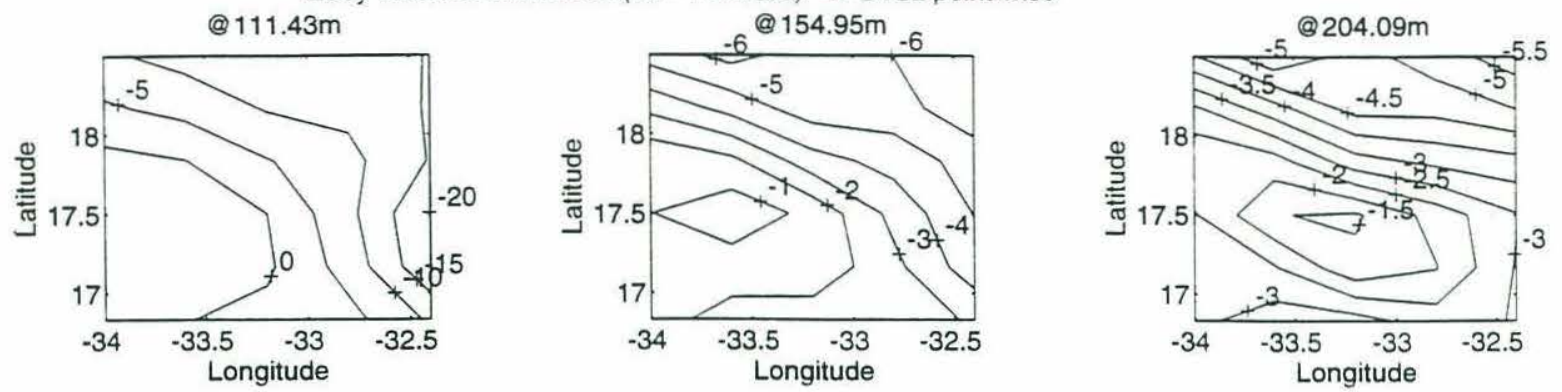

Statistical Errors of ASz (10几1 $\left.\mathrm{cm}^{\wedge} 2 / \mathrm{s}\right):-w^{*} \mathrm{~S}^{*} / \mathrm{Sz}$ point-wise
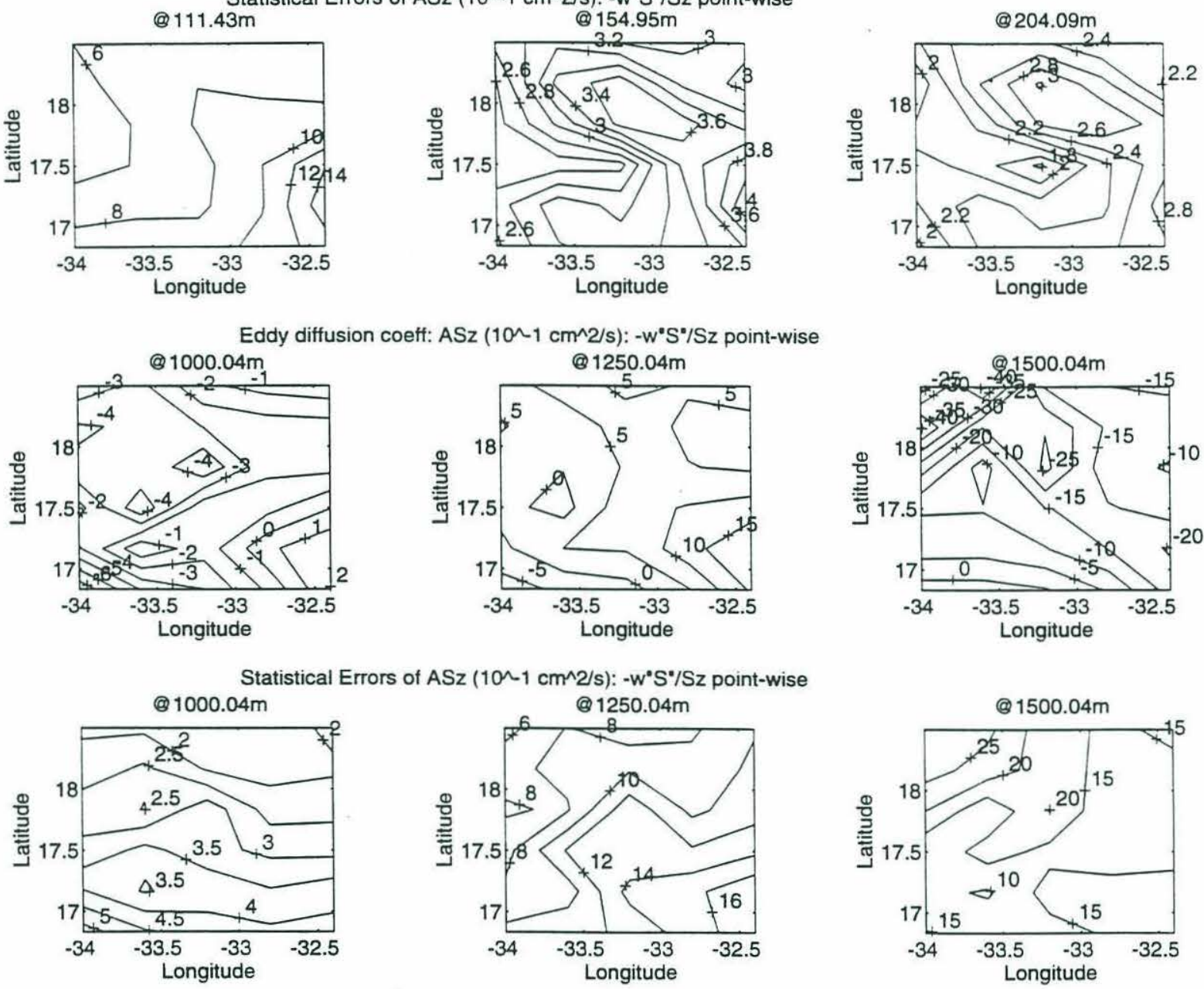

Fig. 4.10 (b) 


\section{ii) "Eddy diffusion" coefficients estimated by the inverse model}

In the inverse model, the horizontal and vertical diffusion coefficients ( $A$ and K) are parameterized as different two-dimensional, third-order, discrete Tchebychev polynomial functions whose coefficients vary from depth to depth. The estimated horizontal diffusion coefficients by the inversion of the time-mean fields of $\mathrm{T}$ and $\mathrm{S}$ are shown in Fig. 4.11 at six depths as examples. Compared with Fig. 4.9, it can be seen that the inverse model estimated values for the horizontal diffusion coefficients are in the same order and magnitudes as those directly calculated from the GCM eddy fluxes at upper levels, although the inverse model estimates are little smaller. At $133 \mathrm{~m}$, the inverse model estimates are consistent with the values of $A_{x}^{T}$ within the errors. At $179 \mathrm{~m}$, there are some significant differences at the southwest corner. At deeper depths, the estimates are much smaller than the values of $A_{x}^{T}$ but without statistical significance.

Shown in Fig. 4.12 are some examples of the inverse model estimated vertical diffusion coefficients. On the upper levels, the inverse model estimates have larger absolute values than those of the direct computations (Fig. 4.10). At greater depths, the estimates are in the same order as the direct computations, but the spatial distribution patterns are obviously different.

The differences between the inverse model estimations and the direct computations (the numerical GCM "data") are due to the discrepancies in the physics

of the inverse model and those of the numerical GCM. The balance terms in the conservations for heat and salt (Fig. 4.3) show that the temporal variation term and the biharmonic dissipation term are important, while they are neglected in the inverse model. To conserve the heat and salt, the neglected terms must be fit into 
other terms, and therefore biases the solutions for the parameters from their "true" values (the "data"). In other words, the inverse model estimated "diffusion" coefficients are not truly the eddy diffusion coefficients - they also parameterize other physical terms which are not explicitly included in the inverse model. At $233 \mathrm{~m}$, the vertical eddy flux terms are negative (Fig. 4.3) and they introduce negative vertical diffusion coefficients (see values at $204 \mathrm{~m}$ and at $261 \mathrm{~m}$ in Fig. 4.9). The temporal variation term at this depth (233 meter, Fig. 4.3) has larger negative values in the north, and large positive value in the south. As this term is not explicitly included in the conservation equation in the inverse model, these large scale signals must be picked up by the terms of the conservation equation of the inverse model, to conserve heat and salt.

If the values of this term are picked up by the vertical "diffusion" terms, they would generate negative vertical diffusion coefficients in the north, and positive diffusion coefficients in the south. The estimated vertical diffusion coefficients at $204 \mathrm{~m}$ and $261 \mathrm{~m}$ have such kind of structures. 
Estimated horizontal diffusivity $A\left(10^{\wedge} 7 \mathrm{~cm}^{\wedge} 2 / \mathrm{s}\right) .2 / 5$ by $1 / 3$ degrees.
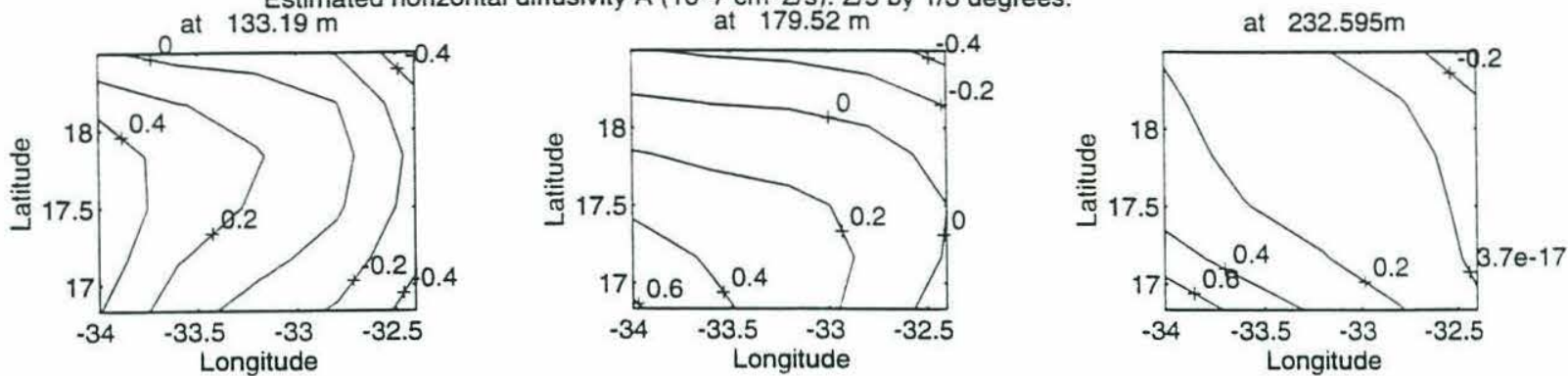

Estimated errors for horizontal diffusivity $A\left(10^{\wedge} 7 \mathrm{~cm}^{\wedge} 2 / \mathrm{s}\right) .2 / 5$ by $1 / 3$ degrees resolution.
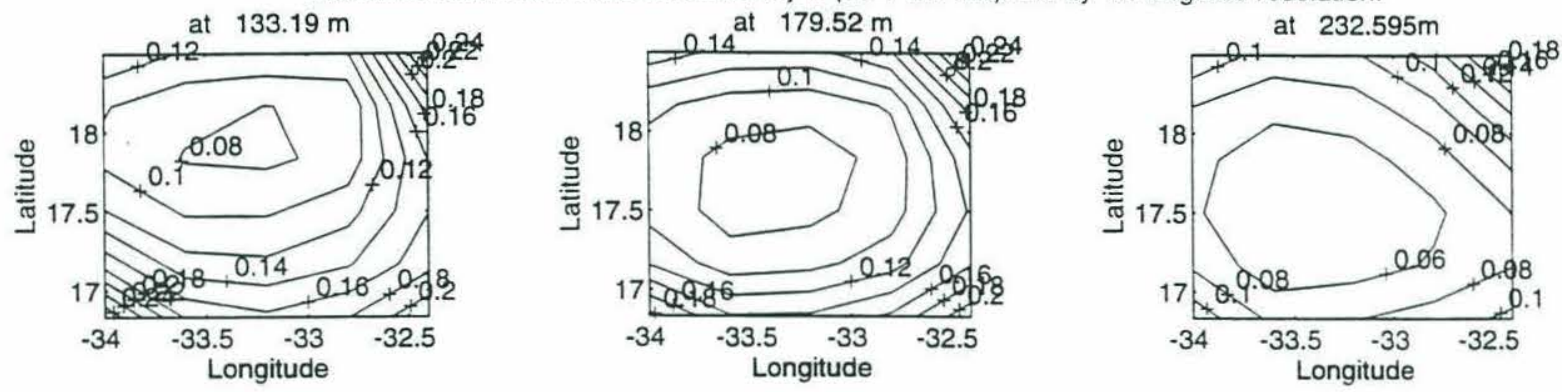

Estimated horizontal diffusivity $\mathrm{A}\left(10^{\wedge} 7 \mathrm{~cm}^{\wedge} / / \mathrm{s}\right)$. $2 / 5$ by $1 / 3$ degrees resolution.
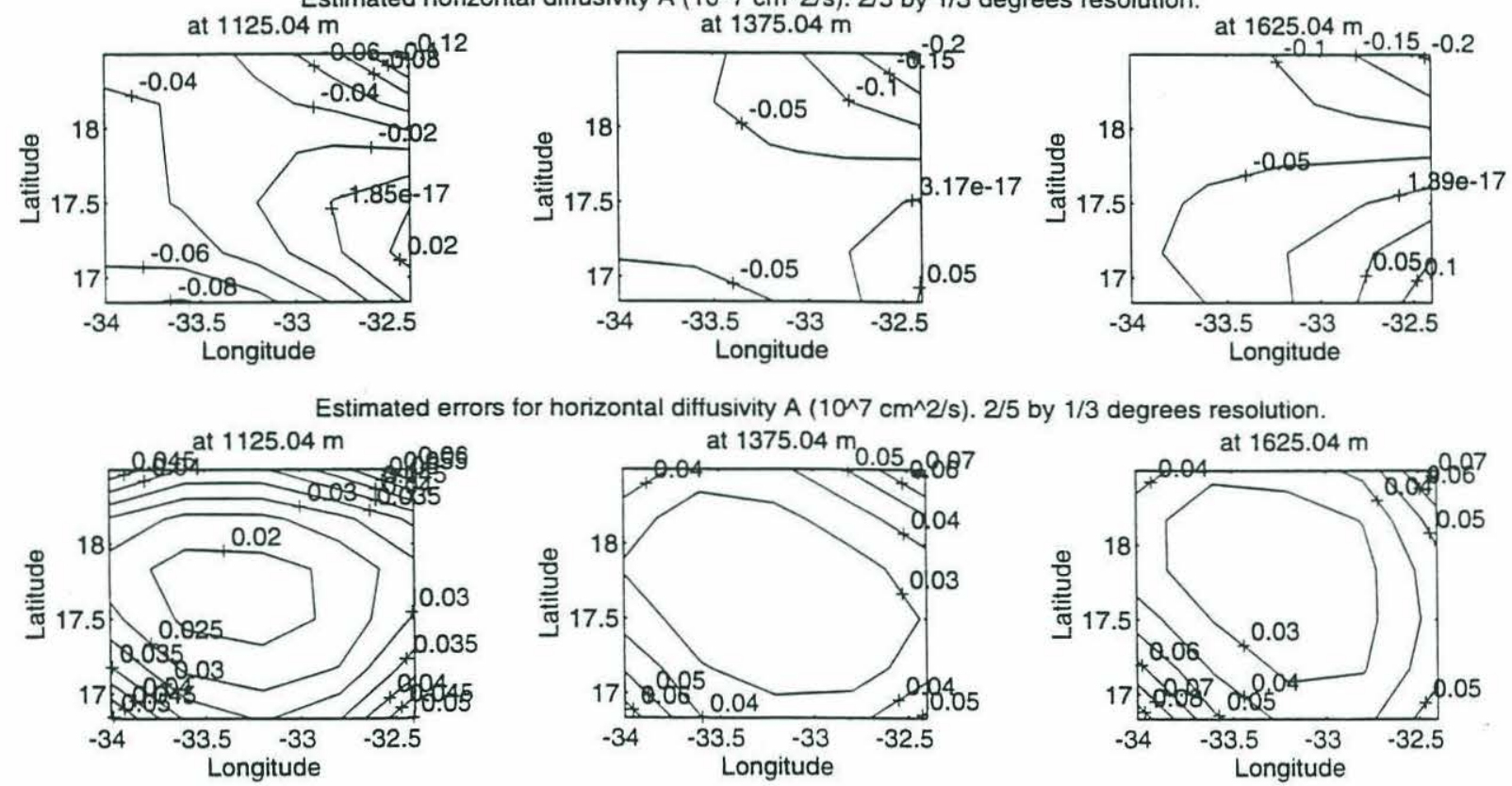

Fig. 4.11. Same as Fig. 4.6 but for horizontal diffusion coefficients $\left(10^{7} \mathrm{~cm}^{2} / \mathrm{s}\right)$. 
Estimated vertical diffusivity $\mathrm{K}\left(10 \wedge-1 \mathrm{~cm}^{\wedge} 2 / \mathrm{s}\right) .2 / 5$ by $1 / 3$ degrees resolution
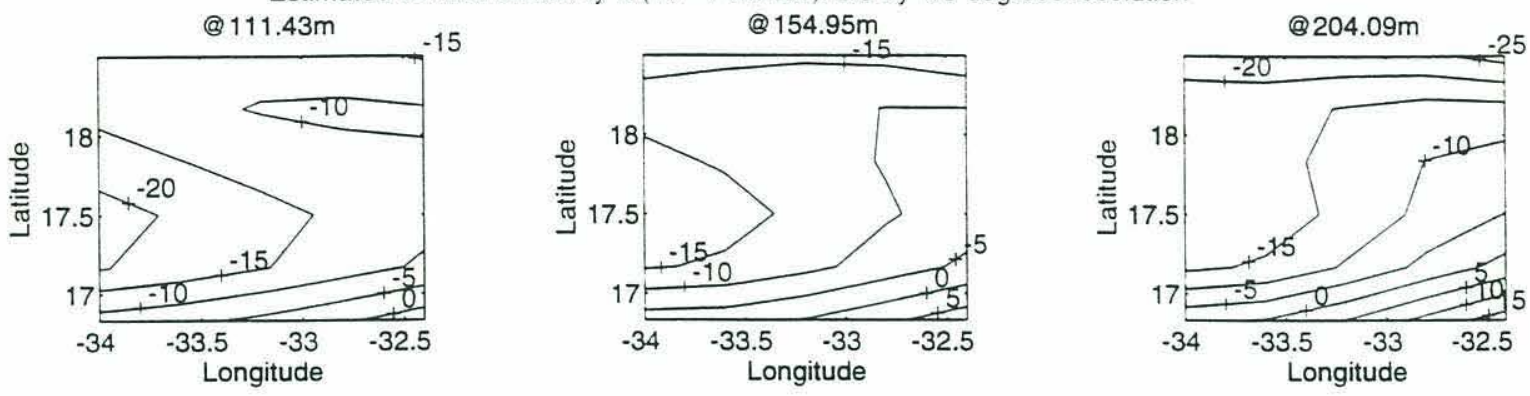

Estimated errors for vertical diffusivity $\mathrm{K}\left(10 \sim-1 \mathrm{~cm}^{\wedge} 2 / \mathrm{s}\right) .2 / 5$ by $1 / 3$ degrees resolution
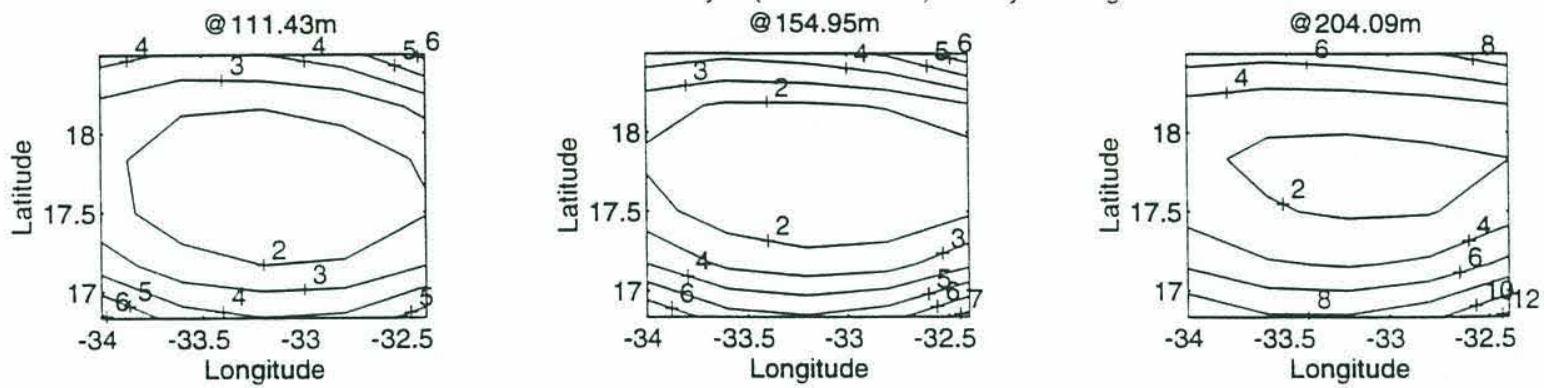

Estimated vertical diffusivity $\mathrm{K}\left(10 \sim 1 \mathrm{~cm}^{\wedge} 2 / \mathrm{s}\right) .2 / 5$ by $1 / 3$ degrees resolution
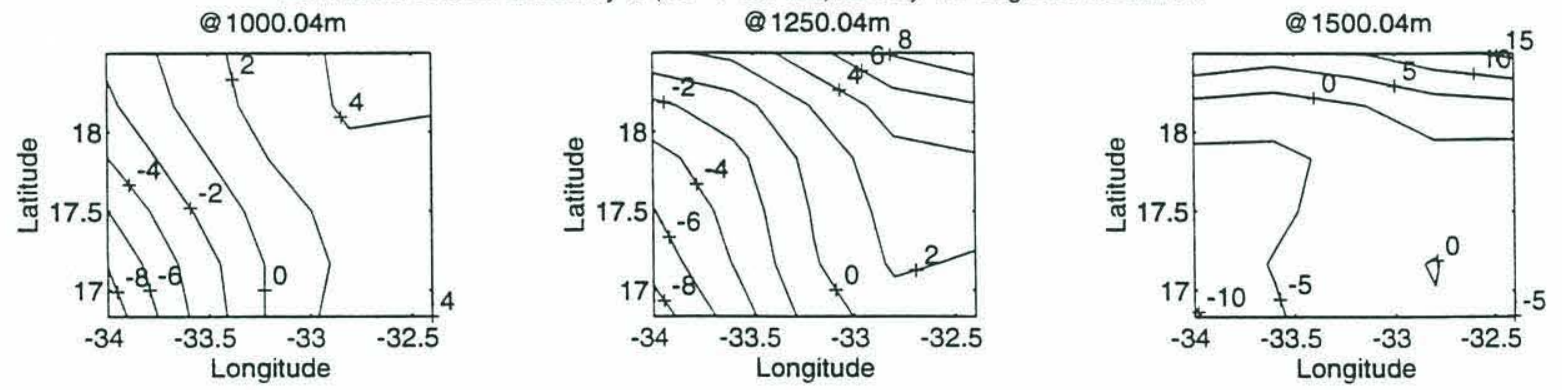

Estimated errors for vertical diffusivity $\mathrm{K}\left(10 \Omega 1 \mathrm{~cm}^{\wedge} 2 / \mathrm{s}\right) .2 / 5$ by $1 / 3$ degrees resolution
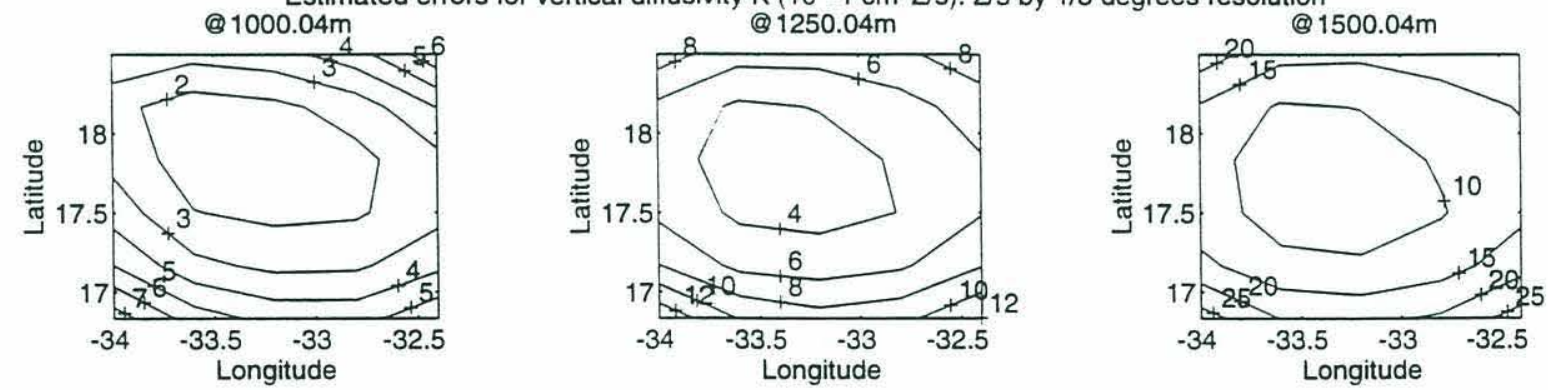

Fig. 4.12. Same as Fig. 4.6 but for vertical diffusion coefficients $\left(\mathrm{cm}^{2} / \mathrm{s}\right)$. 


\subsubsection{Summary and Discussion}

In this section, the inverse model was applied in the time-mean fields of the numerical EGCM ocean, using the same GCM fine grid resolution. It was first shown that part of the assumptions and hypothesis used in the inverse model, namely the thermal wind relation and the continuity equation, are quite accurate in the GCM time-mean fields. However, the other assumptions of the inverse model, namely the steady state conservation equations for heat and salt, are not well satisfied by the same data. For the 5-year means, the temporal variation terms are important players in the heat and salt conservations: their (absolute) values are even larger than those of the dissipation and eddy flux terms, especially of those in the vertical direction. Also, the biharmonic horizontal dissipation terms have well organized spatial structures and large-amplitude signals (compared to other terms) in the fine grid-resolution model.

From the GCM time-mean fields, the inverse model estimated horizontal circulations are very similar to those time-mean ocean circulations of the numerical GCM. They have the same flow patterns throughout the water column in the domain concerned, and numerically they are indistinguishable within the statistical errors.

The inverse model estimated vertical velocities are also statistically consistent with those of the GCM ocean. In terms of the (absolute) closeness to their numerical GCM data values (regardless of the errors), the inverse model solutions for the vertical velocity are not as good as those for the horizontal circulations. The reason is that, in addition to the constraints applied to the vertical velocity (the conservation equations for heat and salt, which are inaccurate in the timemean fields of the numerical GCM ocean, and the accurate continuity equation), 
the horizontal circulations are further constrained by the more accurate dynamic equations (thermal wind balances). Further, small changes in the values of the horizontal circulation (although they might not be significant compared to the circulation themselves) may result in big changes in the divergence of the horizontal circulation, and thus result in large changes in the values of the vertical velocity through the constraint of the continuity equation.

Although the eddy diffusion coefficients directly computed from the GCM eddy fluxes showed that the vertical ones are not the same for heat and salt at great depth, the current accuracy of the steady state conservation equations of the inverse model in the GCM ocean does not allow us to resolve these differences. In the inverse model, the horizontal and vertical diffusion coefficients are assumed to be isotropic and are the same for heat and salt, and they are parameterized as third-order discrete Tchebychev polynomial functions with varying coefficients from depth to depth.

The inverse model solutions for the horizontal "diffusion" coefficients show that they are of the same order and magnitude as those directly computed from the GCM eddy fluxes, but the values and spatial distribution patterns are not the same. The estimated values of the vertical diffusion coefficients are of the same order as those directly computed from the GCM eddy fluxes in the upper levels, but with much larger values at great depths. The absolute values (as the vertical diffusion coefficients are mostly negative) of the inverse model solutions are generally larger than the direct computations.

The differences between the inverse model solutions and those parameter values of the GCM ocean are accounted for by the fact that the diffusion coefficients 
not only parameterize the intended eddy flux terms in the conservation equations of heat and salt, they also partly pick up the signals which are important in the conservation equations but not explicitly included in the inverse model equations. These "missing" signals also affect the solutions for the horizontal circulations, but to a less extent. 


\subsection{APPLICATION OF THE INVERSE MODEL IN THE SPATIALLY SMOOTHED TIME-MEAN FIELDS OF THE EDDY-RESOLVING GCM OCEAN}

\subsubsection{Introduction}

In Section 4.3, the inverse model was tested in the time-mean fields of the EGCM ocean, using the same fine grid-resolution as in the EGCM $\left(2 / 5^{\circ}\right.$ longitude by $1 / 3^{\circ}$ latitude). In applying the inverse model in the real ocean to study the large scale climatological mean ocean circulations, it is neither practical, nor necessary to use such small grid spacing.

As shown in the previous section, it is impossible to do the SVD inversion in a large domain with such small grid spacing, due to the limited memory of the computer resource (the $\mathrm{CPU}$ time is not a serious problem in this case). One remedy to this problem, if there is enough justification to use the grid resolution as high as the above, is to do the inversion block by block. As experiments have shown that the inverse model results are quite insensitive to the horizontal size of the inverse model domain, the inverse model results in the individual blocks can be synthesized together to compose the large scale circulations in the ocean. Also, if detailed analysis of the eigenvalues and eigenvectors are not necessary, other methods, especially those specialized in dealing with sparse matrices, can be utilized.

However, at the present spatial occupation of hydrographic stations in the world oceans, the stations are not dense enough to allow necessary computations and inversions in such fine grid resolutions in most (especially in the deep part) of 
the oceans. In composing the climatological atlas of the oceans, objective mapping and spatial smoothing are usually applied (e.g., Levitus, 1982, Lozier et al, 1994). These spatial smoothing processes usually filter out the small scale structures of the raw data, and only pick up the large scale structures. Computations and inversions with grid resolutions much smaller than the scales over which the smoothing was done are usually not necessary.

When the inverse model is applied to the spatially smoothed climatological mean hydrographic data in a coarse grid resolution, the interpretation of the inverse model results becomes more complicated. Firstly, the order of doing inversion and the order of taking spatial smoothing are not commutable. Also, when coarse grid resolution is used, the issue of subsampling aliasing will also arise (see next section for detail). On the other hand, in applying the inverse model to the spatially smoothed climatological time mean hydrographic data, the inverse model solutions for the circulations are usually interpreted as the spatially smoothed (large scale) climatological mean ocean circulations. Also, the solutions for the diffusive parameters are usually interpreted as the eddy diffusion coefficients. In this section, using the available data in the EGCM ocean (which are not systematically available in the real ocean), we will examine how accurate the above hypotheses are, through the examination of the inverse model results in the context of the spatially smoothed time-mean numerical GCM ocean data. 


\subsubsection{Subsampling Aliasing - the Accuracy of the Inverse Model in a Coarse-grid Resolution}

For generating the spatially smoothed data, the 5-year, 600-sample timemean data of the EGCM ocean are carved in a region from $44.4^{\circ} \mathrm{W}$ to $20.81^{\circ} \mathrm{W}$, $6.83^{\circ} \mathrm{N}$ to $28.5^{\circ} \mathrm{N}$, and from $91 \mathrm{~m}$ to $2125 \mathrm{~m}$ vertically. This results in a total grid number of $60 \times 66 \times 15$ in the fine grid resolution (of $2 / 5^{\circ}$ longitude by $1 / 3^{\circ}$ latitude). A horizontal Gaussian smoothing, with radius of six points $\left(2.4^{\circ}\right.$ in the zonal direction, and $2.0^{\circ}$ in the meridional direction), is applied to the center part of the above carved region, generating a smoothed data set of grid spacing of $2.4^{\circ}$ longitude by $2.0^{\circ}$ latitude. The total number of grids for the smoothed data set is $9 \times 10 \times 15$ in this coarse grid resolution, and the horizontal domain extends from $42.2^{\circ} \mathrm{W}$ to $23.0^{\circ} \mathrm{W}, 8.67^{\circ} \mathrm{N}$ to $26.67^{\circ} \mathrm{N}$.

When the data are decomposed into the spatially smoothed fields and the perturbations from them, "extra" terms (associated with the perturbations) in addition to the terms associated with the smoothed fields arise in the finite difference equations of the GCM in the coarse grid resolution. The major mechanism for the appearance of the extra terms is the subsampling aliasing effects of the large grid spacing. Another possible mechanism is subtle difference in the exact schemes used in the spatial smoothing and the finite difference spatial gradient/divergence operators. The principles of the two mechanisms are shown in Appendix A with illustrations by simple examples. Also as shown in Appendix A, the subsampling aliasing effects are greatly reduced by the spatial smoothing. In Killworth and Bigg's (1988) coarse grid resolution cases, there was no indication of the usage of spatial smoothing. This might lead to significant biases for the parameter solutions. 
As a simple demonstration, Fig. 4.13 shows how the gradient/divergence resulting from the smoothed data might differ from that resulting from the "raw" data in different grid resolutions. Shown in this figure is the time-mean temperature profile of the EGCM ocean in the fine grid resolution of $2 / 5^{\circ}$ longitude (dotted lines) at $1875 \mathrm{~m}$ depth along $17.5^{\circ} \mathrm{N}$. Also shown in this figure is the corresponding Gaussian smoothed temperature profile with a smoothing radius of $2.4^{\circ}$ longitude (dashed lines). Between $25^{\circ} \mathrm{W}$ and $22.5^{\circ} \mathrm{W}$ along this section, if the finite temperature gradients are taken over a distance of $2.4^{\circ}$ longitude, both the smoothed profile and the unsmoothed "raw" data result in similar zonal gradients, but they are not the same. However, if the gradient and the associated equations are written in a smaller grid spacing (say the original $2 / 5^{\circ}$ longitude), the results from the smoothed field and those from the "raw" data are very different: the smoothed field has relatively small negative gradients (slow down-slopes), while the "raw" data have much large positive gradients (steep up-slopes).

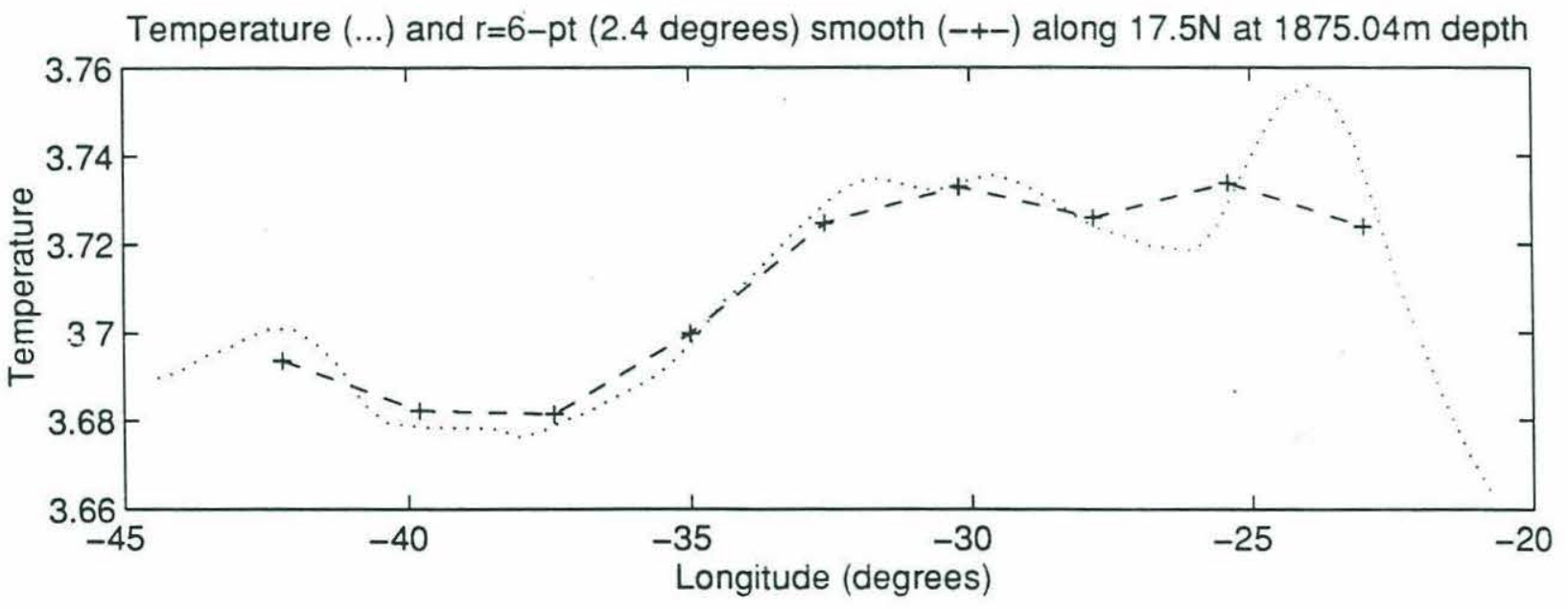

Fig. 4.13. Temperature profiles along $17.5^{\circ}$ at $187.5 \mathrm{~m}$ depth. Dotted line: data on the EGCM fine grid resolution. Dashed line: spatially smoothed data. 
Noticing the subsampling aliasing effects, we will first examine how accurate the inverse model equations are for the spatially smoothed time-mean fields of the EGCM ocean in the coarse grid resolution. For easy formulation, define the primed quantities as the perturbations of the unsmoothed time-mean quantities from the spatially smoothed time-mean quantities:

$$
T^{\prime}=T-\bar{T}^{s} .
$$

In this section, for easy readability, all the variables without the overbars represent the time-mean quantities defined in previous sections (with an overbar in those sections), and the variables with overbars (also labeled with " $s$ ") in this section represent the spatially smoothed quantities.

\section{Continuity Equation}

Without spatial smoothing, the time-mean velocity fields $(u, v, w)$ of the numerical EGCM ocean satisfy the continuity equation exactly on the fine grid resolution $\left(2 / 5^{\circ}\right.$ longitude by $1 / 3^{\circ}$ latitude):

$$
\frac{\partial u}{\partial x}+\frac{\partial v}{\partial y}+\frac{\partial w}{\partial z}=0
$$

Note that as defined before, in this section all the variables without overbars are the 5 -year time means instead of the instantaneous data. Substituting $u$ by $\bar{u}^{s}+u^{\prime}, \quad v$ by $\bar{v}^{s}+v^{\prime}$, and $w$ by $\bar{w}^{s}+w^{\prime}$, then the continuity equation for the spatially smoothed 
time-mean velocities is

$$
\frac{\partial \bar{u}^{s}}{\partial x}+\frac{\partial \bar{v}^{s}}{\partial y}+\frac{\partial \bar{w}^{s}}{\partial z}=-\left(\frac{\partial u^{\prime}}{\partial x}+\frac{\partial v^{\prime}}{\partial y}+\frac{\partial w^{\prime}}{\partial z}\right)
$$

In the inverse model equations for the spatially smoothed quantities, the perturbation terms on the right hand side are unknown (neglected) and they serve as the data "noises" for the smoothed quantities. Direct computations of the terms $\partial \bar{u}^{s} / \partial x, \partial \bar{v}^{s} / \partial y$, and $\partial \bar{w}^{s} / \partial z$ using the coarse grid resolution of $2.4^{\circ}$ longitude by $2.0^{\circ}$ latitude, are shown in Fig. 4.14 for several depths, together with their residuals (or the divergence of the perturbation velocities in eq.(4.23)). From this figure it can be seen that at shallower depths (e.g., at $133 \mathrm{~m}$ ), the spatially smoothed horizontal velocities have larger individual divergence terms than other terms in the figure, and their sum (the total horizontal velocity divergence) is in the same magnitude as the spatially smoothed vertical velocity divergence term, which in turn is larger than the sum of the three individual velocity divergence terms, i.e. the total threedimensional velocity divergence (i.e. $a b s\left(\partial \bar{u}^{s} / \partial x\right) \sim a b s\left(\partial \bar{v}^{s} / \partial y\right)>a b s\left(\partial \bar{w}^{s} / \partial z\right)$, and $a b s\left(\partial \bar{u}^{s} / \partial x+\partial \bar{v}^{s} / \partial y\right) \sim a b s\left(\partial \bar{w}^{s} / \partial z\right)>a b s\left(\partial \bar{u}^{s} / \partial x+\partial \bar{v}^{s} / \partial y+\partial \bar{w}^{s} / \partial z\right)$. Thus the continuity equation is approximately satisfied by the spatially smoothed velocities in this coarse grid resolution. However, at great depths (e.g. at 1625 $\mathrm{m})$, although the individual horizontal velocity divergence terms of the spatially smoothed fields $\left(\partial \bar{u}^{s} / \partial x\right.$ and $\left.\partial \bar{v}^{s} / \partial y\right)$ still have larger values (than the $\partial \bar{w}^{s} / \partial z$ term) and their signs are opposite, their sum (i.e. the total horizontal velocity divergence) cannot be balanced by the spatially smoothed vertical velocity divergence $\left(\partial \bar{w}^{s} / \partial z\right)$, and the total residuals (sums of the total 3-D velocity divergences) are 
significant compared to the vertical velocity divergence $\partial \bar{w}^{s} / \partial z$. It can be seen that the residuals/imbalances have similar large scale horizontal structures as the individual horizontal velocity divergence terms $\left(\partial \bar{u}^{s} / \partial x, \quad \partial \bar{v}^{s} / \partial y\right)$.
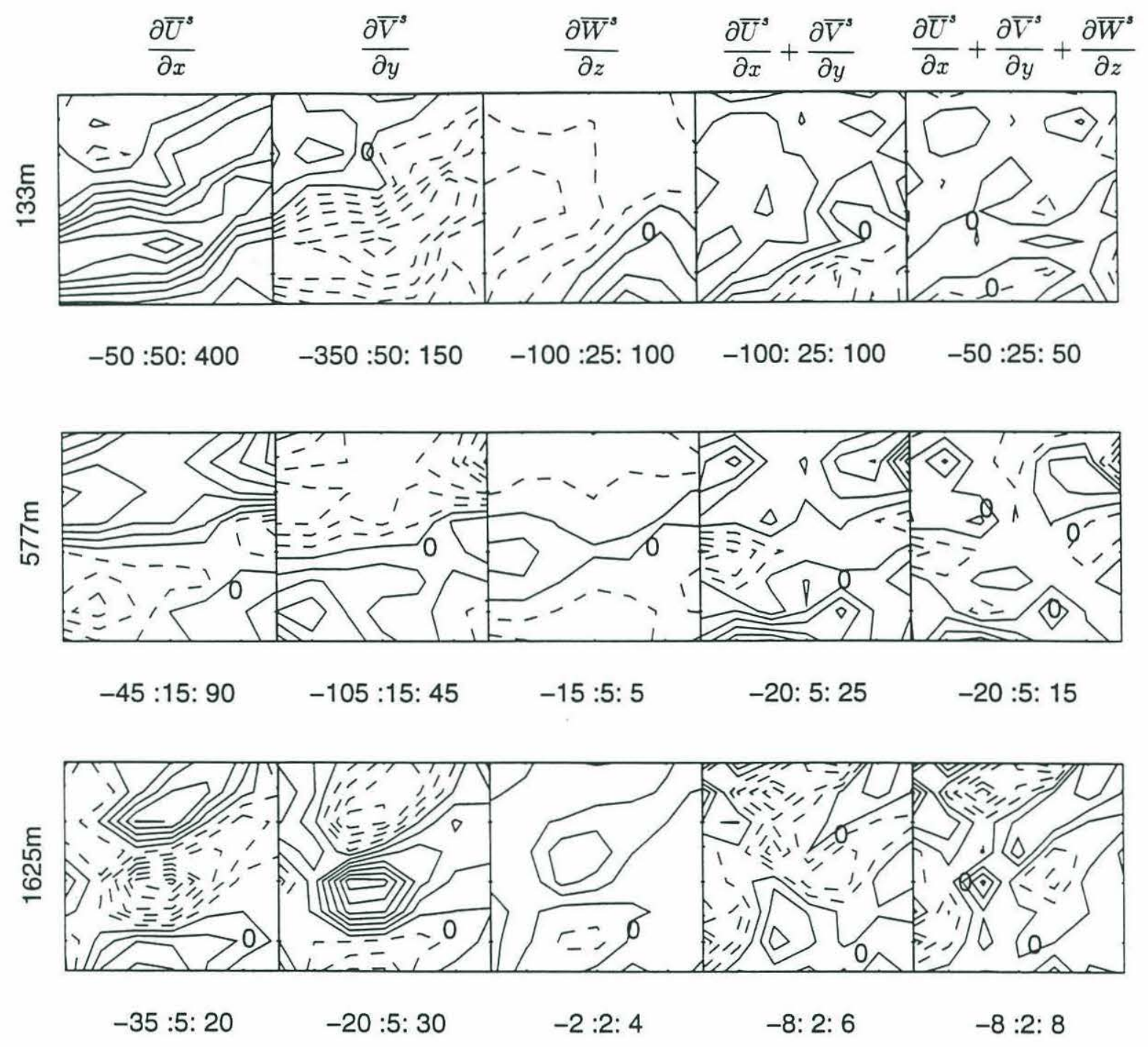

Fig. 4.14. Terms $\left(10^{-11} 1 / \mathrm{sec}\right)$ in the continuity equation for the spatially smoothed time-mean velocity field at three depths. Numbers at the bottoms are the contour values and intervals. The dashed lines are negative, and the solid lines are positive with the ones adjacent to the dashed lines being zero lines. 


\section{The Thermal Wind Relation}

Section 4.3 showed that the 5-year time-mean horizontal circulations of the EGCM ocean are in very good agreement with the thermal wind relation in the fine grid resolution (of $2 / 5^{\circ}$ longitude by $1 / 3^{\circ}$ latitude): the imbalances are around $5 \%$ relative to the vertical shears themselves. The vertical shears of the spatially smoothed horizontal velocities of the EGCM ocean can also be directly computed (denoted by $\bar{U}^{s} r G C M$ ). Also, thermal wind shears (denoted by $\bar{U}^{s} r D y n$ ) can be computed from the spatially smoothed time-mean density field in the coarse grid resolution (of $2.4^{\circ}$ longitude by $2.0^{\circ}$ latitude).

Examples are shown in Fig. 4.15. Also shown in this figure are the imbalances (the third column) between the absolute horizontal velocity shears and the thermal wind shears. Note that again, as in Fig. 4.3, the contour intervals for the imbalances are $1 / 10$ of the contour intervals for the horizontal velocity shear terms (columns 1 and 2). However, compared to the case of the unsmoothed time-mean horizontal velocity shears on the fine grid resolution shown (in Fig. 4.3), the relative imbalances are larger in this case. The area-averaged ratios of the imbalances over the absolute horizontal velocity shears $R_{u}$ and $R_{v}$, as defined in eq.(3.1) and eq.(3.2), are shown in Table 4.3. It can be seen that these numbers are much larger than those shown

in Table 4.2. The relative imbalances are around $11 \%$ zonally (the vertically of $R_{u}$ ), and around $8 \%$ meridionally. These larger numbers (compared to those in Table 4.2) are again caused by the subsampling aliasing of the large grid spacing (e.g., in computing the thermal wind shears from the density field). Computations showed that these numbers are even larger if larger grid spacing is used. Although not as accurate as for the unsmoothed time-mean horizontal circulations in the fine grid 
resolution, the spatially smoothed time-mean horizontal circulations on the coarse grid resolution are still in approximate thermal wind balance.

Table 4.3 Horizontally averaged relative imbalances between the absolute velocity shears and the thermal wind shears.

$\begin{array}{lllllllllllll}\text { Depth range }(\mathrm{m}) \\ \\ \text { 133-180-233-295-370-463-577-722-901-1125-1375-1625-1875 } \\ R_{u} & 0.09 & 0.05 & 0.06 & 0.09 & 0.10 & 0.07 & 0.07 & 0.09 & 0.17 & 0.22 & 0.19 & 0.15 \\ R_{v} & 0.09 & 0.07 & 0.06 & 0.05 & 0.05 & 0.06 & 0.06 & 0.07 & 0.13 & 0.09 & 0.11 & 0.09\end{array}$

With the spatially smoothed time-mean temperature and salinity fields, the terms in the heat and salt conservation equations are computed in the coarse grid resolution, and some examples are shown in Fig. 4.16. The conventions in this figure are the same as those used in Fig. 4.4 and described in section 4.3.2.

First of all, with the larger grid spacing, the biharmonic horizontal dissipation terms are greatly reduced, and they are much smaller than all the other terms and are insignificant compared to the residuals. Secondly, the temporal variation terms associated with the limited time period over which the time means were taken are still important in these conservation equations. Their values are as large as or even larger than those of the dissipation terms and the eddy flux terms. Thirdly, the heat and salt conservation equations are not balanced by the terms of the spatially smoothed time-mean temperature and salinity fields (the first seven columns in Fig. 4.16). The imbalances come from two sources. One is the source for the unsmoothed 
time-mean temperature and salinity fields in the fine grid resolution as discussed in section 4.3 , namely the subsampling aliasing in the time domain. The other source is associated with the subsampling aliasing in space in this coarse grid resolution.

The conservation equations for heat and salt in the inverse model only include the advection terms and the eddy flux terms, which are parameterized as eddy diffusion terms, in both horizontal and vertical directions (the specified vertical dissipation terms $K_{m} T_{z z}$ in the GCM ocean are also parameterized in the vertical diffusion terms. The horizontal biharmonic dissipation terms are unimportant in this case). The "missing", significantly meaningful signals (e.g., the temporal variation terms) are not explicitly included in the inverse model, and this will possibly bias the inverse model solutions from their "true" values. 

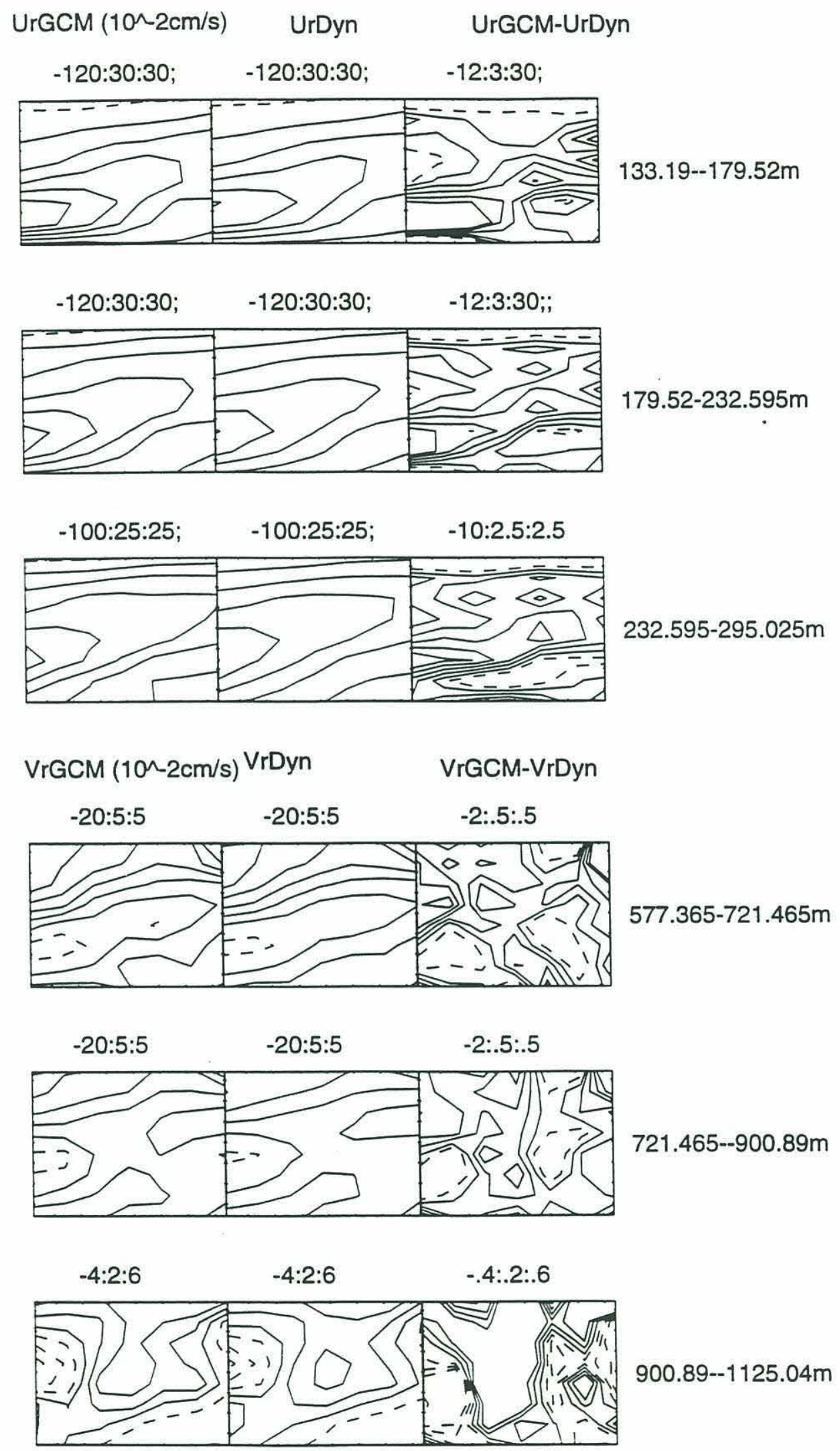

Fig. 4.15. Absolute velocity shears (UrGCM), thermal wind shears (UrDyn), and their differences for the smoothed EGCM data in the coarse grid resolution. 

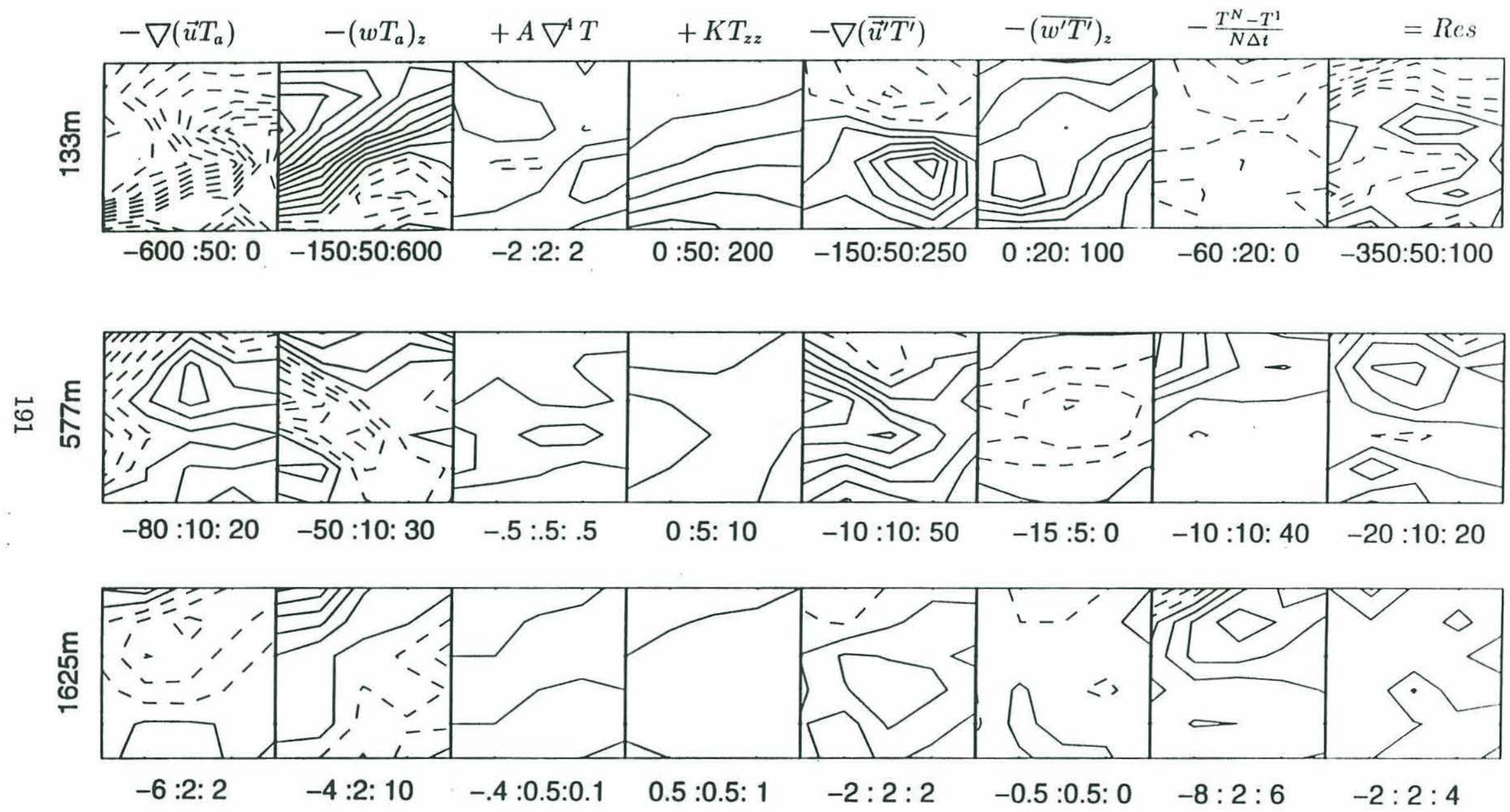

Fig. 4.16. Terms $\left(10^{-10} \mathrm{C} /\right.$ sec $)$ in the conservation equation for the spatially smoothed timemean temperature anomaly ( $T_{a}$, anomaly from the area-averaged value at the depth concerned) at three depths. Numbers at the bottoms are the contour values and intervals. 


\section{Estimation of the Statistical Errors of the Spatially Smoothed Quantities}

In order to fully analyze the inverse model results, it is necessary to estimate the statistical errors for the spatially smoothed time-mean quantities of the numerical GCM ocean. These estimations are analogous to the analysis of the errors for the time means but in 2-D space here.

The statistical errors of the time means on the fine grid resolution scheme of the numerical GCM ocean were already computed in section 4.2. The spatial smoothing in this section is taken over a horizontal plane of a radius of 6 points (in the fine grid resolution). In generating a spatially smoothed datum at one point, $12 \times 12\left(=L_{x} \times L_{y}\right)$ points of the "raw" (unsmoothed) time-mean data (in the fine grid resolution) are used. If the involved $L_{x} \times L_{y}$ points of data are totally independent (i.e. the degree of freedom is $L_{x} \times L_{y}$ ), the variances of the spatially smoothed time-mean quantities are $1 /\left(L_{x} \times L_{y}\right)$ of the time-mean variances of the "raw" data. However, the data are usually correlated in space, and the degrees of freedom are usually smaller than the total number of the points used in the smoothing.

To compute the degrees of freedom of the data in space, it is necessary to compute the auto-correlation length scales of the data. Instead of computing the auto-correlations point-wise in the domain concerned, the data along a zonal section at $17.5^{\circ} \mathrm{N}$ (with 60 points of data) are used to estimate an "averaged" auto-correlation length scale in the east-west direction, and the data along a meridional section at $32.8^{\circ} \mathrm{W}$ (with 66 data points) are used to estimated an "averaged" auto-correlation length scale in the north-south direction. These two sections pass through the center of domain concerned. 
The data are first used to calculate the auto-correlation coefficients:

$$
R(r)=\frac{\left\langle\left(T(x)-\bar{T}^{x}\right)\left(T(x+r)-\bar{T}^{x}\right)>\right.}{<\left(T(x)-\bar{T}^{x}\right)^{2}>}
$$

where $T$ is the time mean variable (along the zonal section) and $\bar{T}^{x}$ is the zonal mean of $T$ along that section. Then the calculated auto-correlation coefficients are used to compute the integral length scales as functions of the upper limit of the integral:

$$
\ell_{x}(x)=\int_{0}^{x} R(r) d r .
$$

Similar computations can be carried out along the meridional section to calculate the auto-correlation length scales $\ell_{y}(y)$ in the north-south direction. These calculations are carried out for all the relevant variables and at all the depths concerned. Some examples of the auto-correlation coefficients and the integral length scales as functions of the upper limit of the integral are shown in Fig. 4.17 for the time-mean zonal velocity. For the same reason discussed in section 4.2 for the integral time scale, we define the "typical" integral length scales as the integrals of the auto-correlation coefficients from perfect correlation $(R=1)$ to the first point of no correlation $(R=0)$. The computation results of these integral length scales are shown in Table 4.4 for some variables at all the depths. Note that like $L_{x}$ and $L_{y}$, 
the units of $\ell_{x}$ and $\ell_{y}$ are the grid distances of the (unsmoothed) "raw" time-mean data (namely $2 / 5^{\circ}$ longitude and $1 / 3^{\circ}$ latitude).

Table 4.4 Integral length scales $\left(l x:(2 / 5)^{\circ}\right.$ longitude; $l y:(1 / 3)^{\circ}$ latitude) and the corresponding degrees of freedom for different variables.

\begin{tabular}{|c|c|c|c|c|c|c|c|c|c|c|c|c|c|}
\hline \multicolumn{14}{|c|}{ Depth (meters) } \\
\hline & & & 33 & 95 & 370 & 53 & 7 & 2 & 01 & 125 & 15 & 1625 & 1875 \\
\hline \multirow[t]{2}{*}{$l x$} & 10.6 & 10.7 & 10.8 & 10.7 & 10.5 & 9.8 & 6.7 & 9.6 & 10.3 & 7.5 & 4.7 & 5.4 & 6.4 \\
\hline & 11.7 & 12.4 & 12.5 & 12.4 & 12.3 & 12.2 & 11.7 & 10.5 & 9.3 & 10.0 & 11.3 & 11.4 & 11.4 \\
\hline$d f$ & 1.0 & 1.0 & 1.0 & 1.0 & 1.0 & 1.0 & 1.0 & 1.0 & 1.0 & 1.0 & 1.3 & 1.1 & 1.0 \\
\hline$l_{x}$ & 4.5 & 5.8 & 7.8 & 8.7 & 8.0 & 5.4 & 3.2 & 2.8 & 3.9 & 4.5 & 5.4 & 7.5 & 5.6 \\
\hline$l_{y}$ & 6.1 & 6.2 & 6.9 & 8.2 & 9.1 & 8.8 & 7.6 & 5.1 & 2.2 & 1.6 & 1.4 & 1.2 & 1.4 \\
\hline$d f$ & 1.3 & 1.0 & 1.0 & 1.0 & 1.0 & 1.1 & 1.9 & 2.5 & 4.2 & 5.1 & 4.9 & 4.9 & 4.6 \\
\hline$l_{x}$ & 4.5 & 3.0 & 2.3 & 2.1 & 2.0 & 1.9 & 1.7 & 1.6 & 1.6 & 1.9 & 1.5 & 1.3 & 1.2 \\
\hline$l_{y}$ & 9.5 & 8.5 & 6.9 & 4.4 & 2.9 & 2.5 & 2.4 & 2.1 & 1.1 & 1.0 & 1.4 & 1.4 & 1.1 \\
\hline$d f$ & 1.3 & 2.0 & 2.7 & 3.9 & 6.2 & 7.4 & 8.5 & 10.7 & 20.3 & 19.4 & 17.5 & 19.7 & 27.2 \\
\hline
\end{tabular}

Similar to the case in the time domain (eq. (4.6-4.9)), the degree of freedom is computed as

$$
d f=\frac{L_{x}}{2 \ell_{x}} \frac{L_{y}}{2 \ell_{y}}
$$

but $d f$ is no less than one. The variances for the spatially smoothed time-mean fields can be estimated as $\sigma_{\bar{T}}^{2} s=\sigma_{T}^{2} / d f$ where $\sigma_{T}^{2}$ is the variance of the unsmoothed time mean variable. 
$\mathrm{U}$ along $17.5 \mathrm{~N}$, at depth:
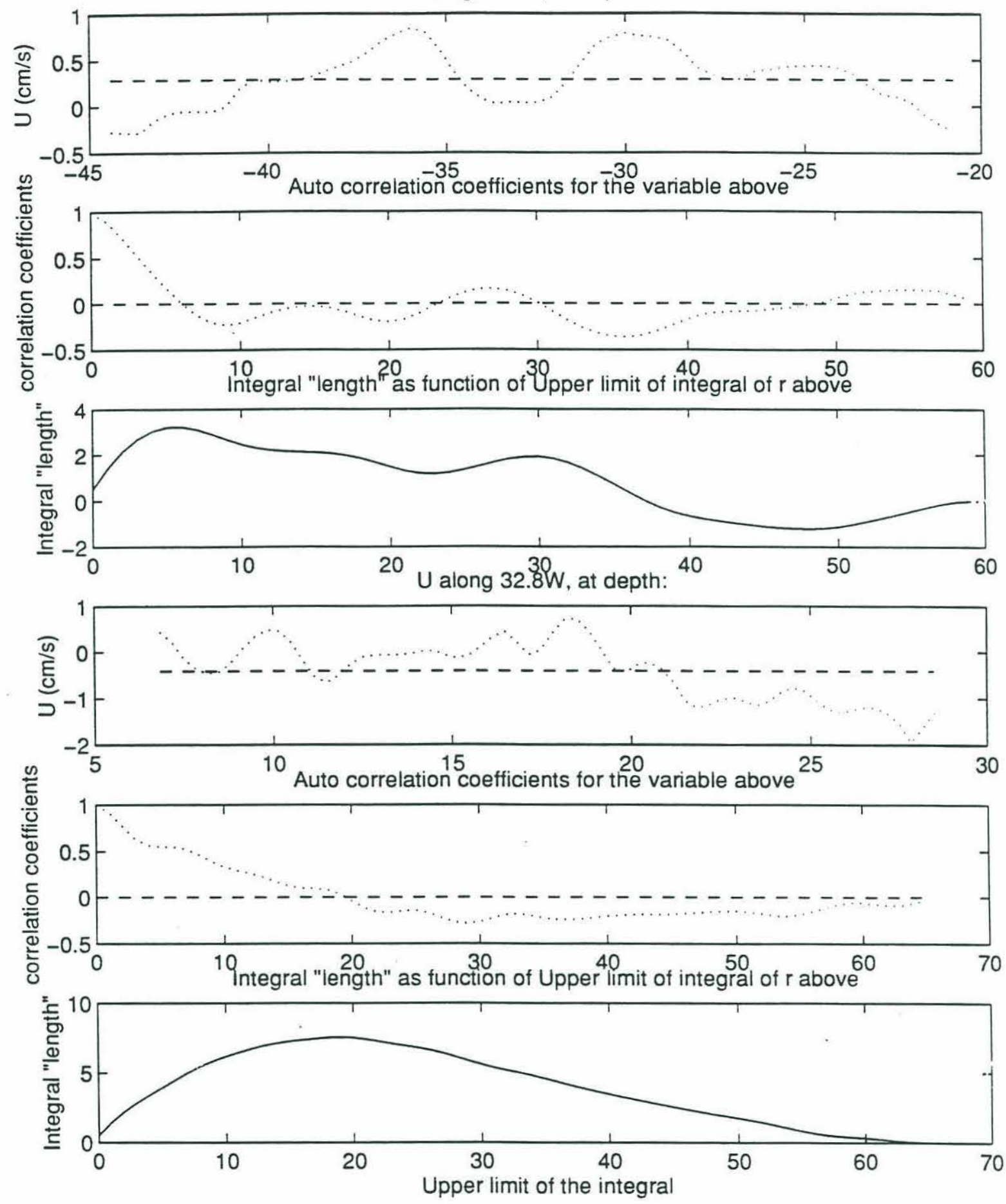

Fig. 4.17. Zonal velocities along $17.5^{\circ} \mathrm{N}$ and $32.80 \mathrm{~W}$. their auto-correlations, as well as the integral lengths (in the unit of grid spacing: $2 / 5^{\circ}$ longitude or $1 / 3^{\circ}$ latitude). 
Table 4.4 shows that the perturbation temperature (and salinity) fields have quite large correlation scales in space, especially in the north-south direction. In fact at most of the depths, $d f=1$, which suggests no independent information is available in the smoothing domain. (In order to reduce the uncertainties, a larger smoothing radius would be used.) However, the integral length scales for the timemean velocities $(u, v$, especially for $w)$ are much smaller, especially at great depths. The statistical errors for the spatially smoothed time-mean temperature and salinity fields are similar to those for the the unsmoothed time means of the GCM ocean, while the statistical errors for the spatially smoothed velocities are greatly reduced, especially for the vertical velocity and at great depths.

\subsubsection{Inverse Model Results in the Spatially Smoothed Time- Mean Ocean}

In this section the inverse model is applied to the spatially smoothed timemean hydrographic data of the EGCM ocean. The grid resolution of $2.4^{\circ}$ longitude by $2.0^{\circ}$ latitude are the same as the radius of the spatial smoothing. The domain over which the inverse model is applied extends from $42.2^{\circ} \mathrm{W}$ to $23.0^{\circ} \mathrm{W}, 8.67^{\circ} \mathrm{N}$ to $26.67^{\circ} \mathrm{N}$, and from $91 \mathrm{~m}$ to $2125 \mathrm{~m}$ in the vertical. The total number of the grid points (on which the equations are formulated) is $9 \times 10 \times 13$. The unknowns are the streamfunctions (for the horizontal velocities), the vertical velocities (the above two variables are treated as point-wise unknowns), and the horizontal and vertical "diffusion" coefficients. The "diffusion" coefficients are purposely introduced to

parameterize the eddy flux terms, but in reality, they also partially parameterize the signals not explicitly included in the inverse model (e.g., the temporal variation terms). The "diffusion" coefficients are parameterized as the third-order discrete 
Tchebychev polynomial functions in the horizontal plane whose coefficients vary from depth to depth. The constraints on these unknowns are the dynamic equations, the continuity equation, and the steady state conservations for heat and salt. These result in 3036 equations for 1990 unknowns in the domain described above.

The singular value profile (Fig. 4.18a) of the coefficient matrix of the equation system and the Levenberg-Marquardt diagram (Fig. 4.18b) described in section 3.3.4 show that this equation system is of full rank, and all the available information (all the eigenvalues and their corresponding eigenvectors) are used in obtaining the solutions for the unknowns. The equation system is over-determined in the traditional sense, and the inverse model solutions are obtained by minimizing the residual norm of the equations. The constraint equations are weighted according to their accuracy in the spatially smoothed time-mean fields of GCM ocean. The dynamic equations are most accurate and are given a higher weight, so that they contribute most to the inverse model solutions (Fig. 4.18(c) shows the so-called data resolution, which signifies the contributions of the equations to the inverse model solutions. See Wunsch, 1989 for detail). The conservation equations for heat and salt are less accurate and a smaller weight is given to them. Within the conservation equations, the depth-dependent weighting factor are chosen according Zhang and Hogg (1992). The post-inverse residuals in the weighted equations are in the same order and are more or less randomly distributed (Fig. 4.18(d)). Their autocorrelation coefficients of are vanishingly small except near zero lag (Fig. 4.18e). These are consistent with the a priori assumption used in the estimation that the data noises are white. 

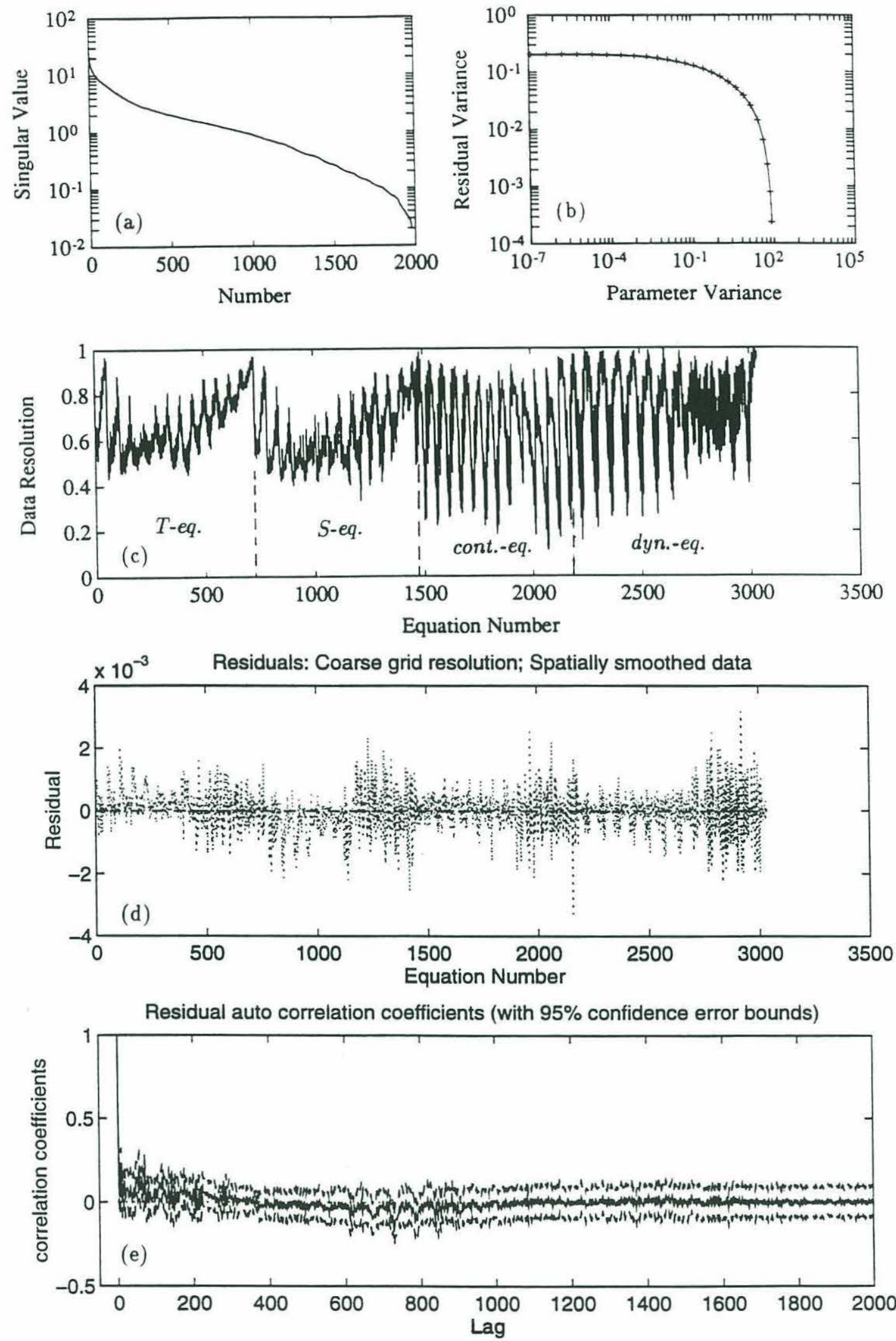

Fig. 4.18. Analyses of the equation system for the spatially time means with coarse grid resolution. (a) Singular value profile; (b) Levenberg-Marquardt stabilization diagram; (c) data resolution; (d) post-solution equation residuals; (e) the residual auto-correlations. 
In the following paragraphs, the inverse model solutions for the unknowns will be analyzed in the context of the spatially smoothed time-mean parameter values of the EGCM ocean.

\section{Horizontal Circulations}

The spatially smoothed time-mean horizontal circulations of the EGCM ocean in the inverse model domain are shown in Fig. 4.19 along with their sta-

tistical error ellipses. Different vector scales are used at different depths for easy visualization of the flow patterns. At shallow depths, the circulations are strong and have well organized flow patterns. However, at great depths (say below 900 $\mathrm{m}$ depth), the circulations are very weak and the flow patterns are generally not well organized. Compared to the unsmoothed time-mean horizontal circulations on the fine grid-resolution scheme of the numerical GCM ocean (Fig. 4.5), it can be seen that the errors for the spatially smoothed time-mean horizontal circulations are greatly reduced.

The horizontal circulations estimated by the inverse model are shown in Fig. 4.20 together with their estimated uncertainties. The vector scales used in this figure are the same as those in Fig. 4.19. Direct comparison of Fig. 4.20 with Fig. 4.19 show that, at shallow depths (say at the eight vertical levels above 722 $\mathrm{m}$ depth), the inverse model estimated circulations are very similar to the spatially smoothed time-mean ocean circulation of the numerical GCM ocean in both pattern and value. In fact, they are consistent with each other within their error ellipses.

Below $900 \mathrm{~m}$ depth, the circulations become very weak. On these depths, the estimated horizontal circulations are consistent with the spatially smoothed time- 
mean EGCM ocean circulations in most of region, although there are significant discrepancies at few points (noticeably at the northwest corner). For example, at the deepest two levels at $1625 \mathrm{~m}$ and $1875 \mathrm{~m}$, although there are some differences between the estimates and the spatially smoothed GCM ocean in the detailed structures of the horizontal circulations, they both result in the similar large scale flow patterns with major flows from the southwest to the northeast.

\section{Vertical Velocity}

Shown in Fig. 4.21 are the spatially smoothed time-mean vertical velocity fields of the numerical GCM ocean at six depths along with their statistical errors. As mentioned in the previous section, the errors are greatly reduced compared to those of the unsmoothed time means, especially at great depths. As pointed out in section 4.3 , the statistical errors of the (unsmoothed) time means of the GCM vertical velocity are so large at the great depths that the values of the timemean vertical velocity themselves are not significantly above these errors (therefore they are indistinguishable from zero). However, the spatially smoothed time-mean vertical velocities shown in Fig. 4.21 are significantly above their errors at the shallow depths, and they are also marginally non-zero at greater depths.

The inverse model estimated vertical velocities are shown in Fig. 4.22. Comparisons of Fig. 4.22 with Fig. 4.21 show that, as in the fine-grid resolution case of the time means, the estimates are statistically consistent with the EGCM data values in most of the domain. The coarse-grid resolution estimated vertical velocities from the spatially smoothed time-mean hydrographic data are also similar to the spatially smoothed time-mean vertical velocities of the EGCM ocean in pattern. 
The agreement is obvious at shallow depths (e.g., at $111.43 \mathrm{~m}, 154.95 \mathrm{~m}$, and 204.09 $\mathrm{m}$ of the upper panel in these figures).

However, as going deeper and deeper, the differences between the inverse estimates and the spatially smoothed time means of the EGCM ocean become more and more apparent at the north-west corner. At $1500 \mathrm{~m}$, the inverse model estimated values at this corner ( of $10 \times 10^{-6} \mathrm{~cm} / \mathrm{s}$ ) are significantly different from the values (of $-50 \times 10^{-6} \mathrm{~cm} / \mathrm{s}$ ) of the spatially smoothed time-mean vertical velocity of the numerical GCM ocean, although the differences in the rest of the area (between 0 and $10 \times 10^{-6} \mathrm{~cm} / \mathrm{s}$ ) are generally insignificant within their error bars. The significant discrepancies between the inversion and the EGCM data values happen at the place (the northwest corner) where the neglected time variation terms in the heat and salt conservation equations show their importance (say at $577 \mathrm{~m}$ in Fig. 4.16). These "missing" signals were partially picked up by the vertical advection terms and thus biased the solutions for the vertical velocity (note that at this corner at $577 \mathrm{~m}$ in Fig. 4.16, magnitude of the advection term is smaller than that of the time variation term). 
GCM horizontal circulations with error ellipses: $2.4 \times 2.0$ degrees smoothed

$133.19 \mathrm{~m}$

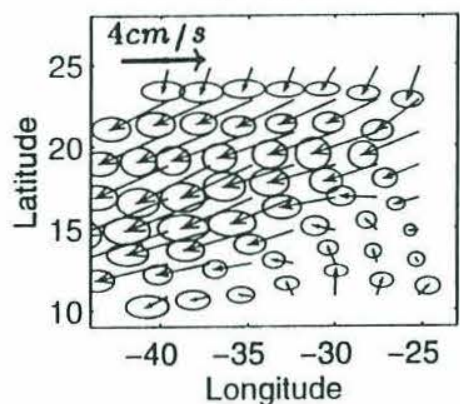

$295.025 m$

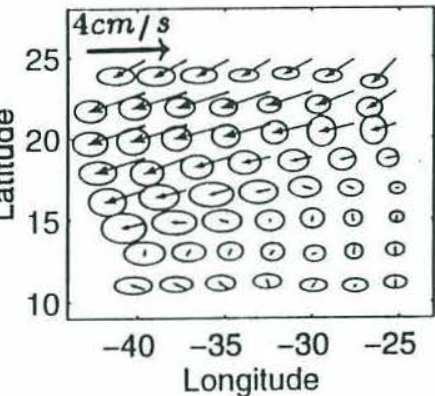

$577.365 \mathrm{~m}$

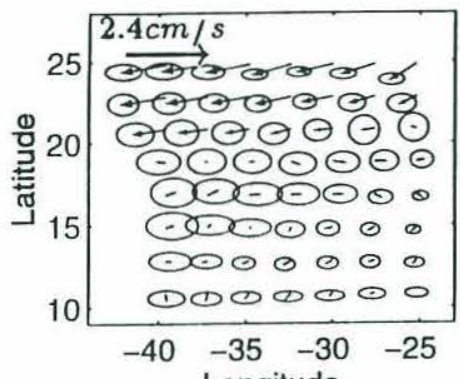

$179.52 \mathrm{~m}$

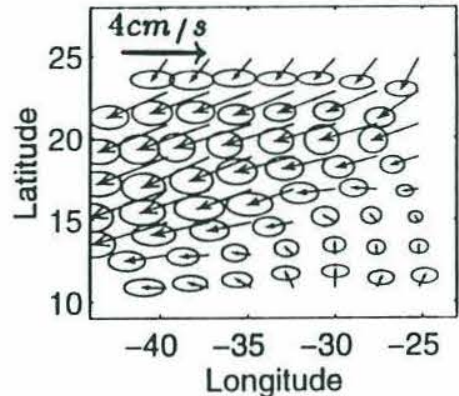

$370.21 \mathrm{~m}$
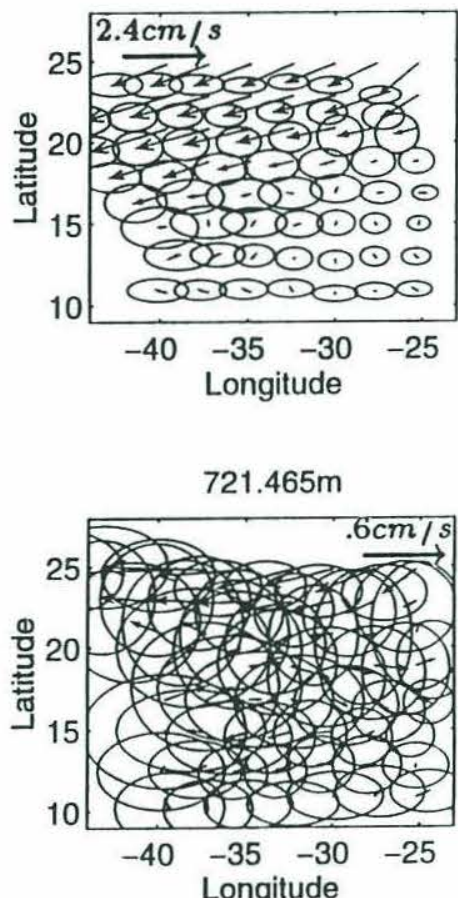

$232.595 \mathrm{~m}$

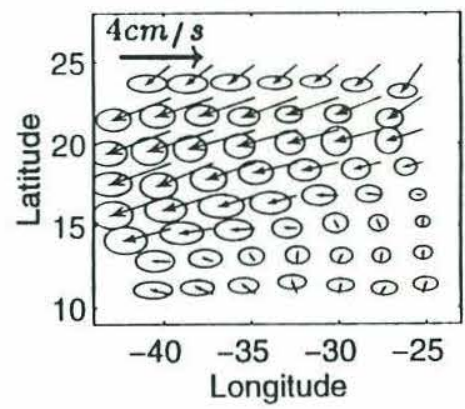

$462.505 \mathrm{~m}$

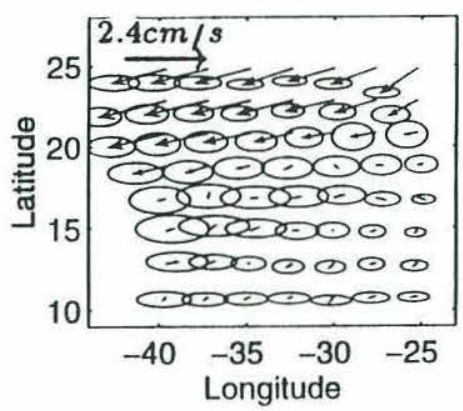

$900.89 \mathrm{~m}$

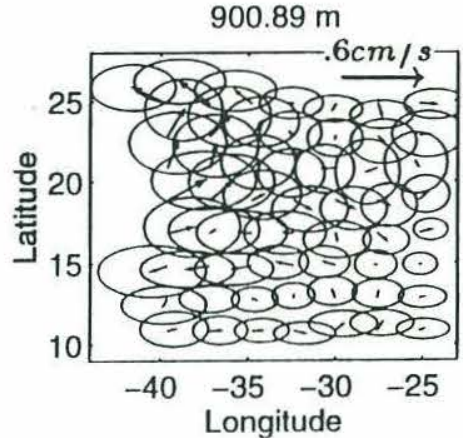

Fig. 4.19. Spatially smoothed time-mean EGCM horizontal circulations with their statistical error ellipses. The arrows are the vector scales. 
GCM horizontal circulations with error ellipses: $2.4 \times 2.0$ degrees smoothed $1125.04 \mathrm{~m} .3 \mathrm{~cm} / \mathrm{s}$

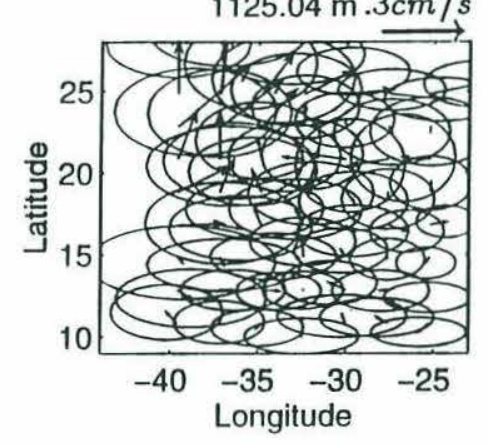

$1375.04 \mathrm{~m} .3 \mathrm{~cm} / \mathrm{s}$
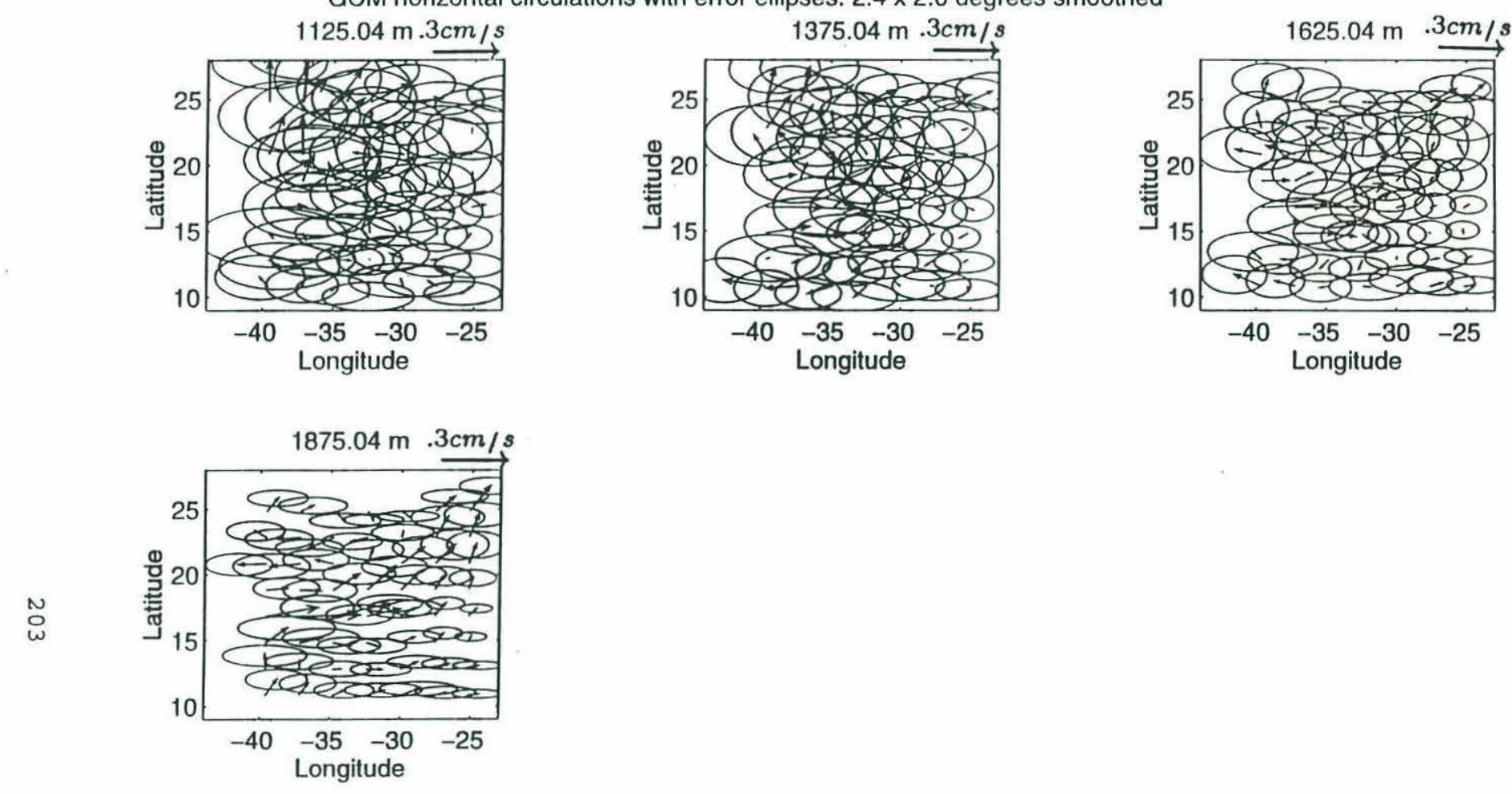

Fig. 4.19 (cont.) 
Estimated horizontal circulations with error ellipses. $2.4 \times 2.0$ degrees resolution, $\mathrm{Nl}=13$

$133.19 \mathrm{~m}$

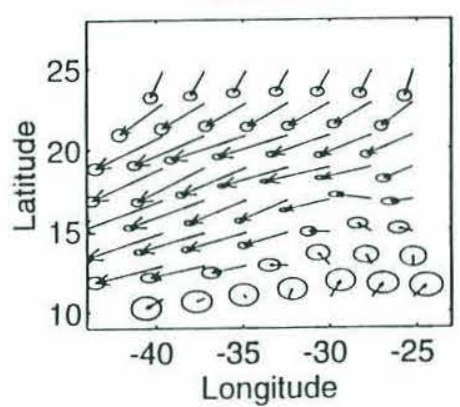

$295.025 m$
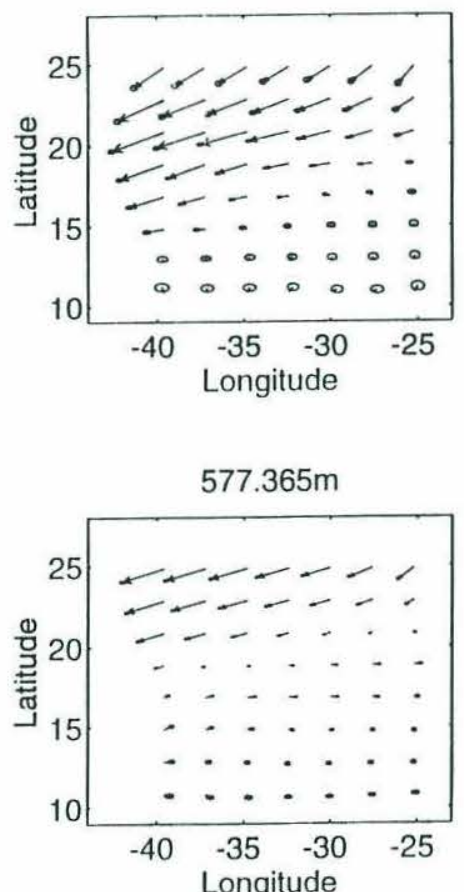

$179.52 \mathrm{~m}$

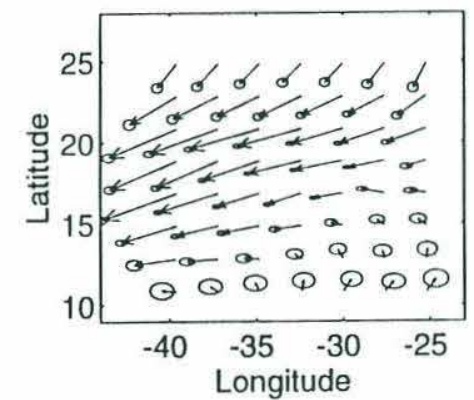

$370.21 \mathrm{~m}$

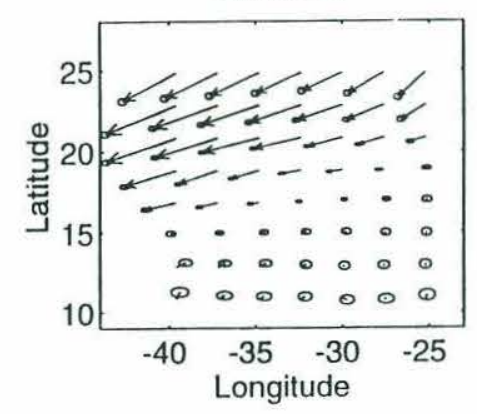

$721.465 \mathrm{~m}$

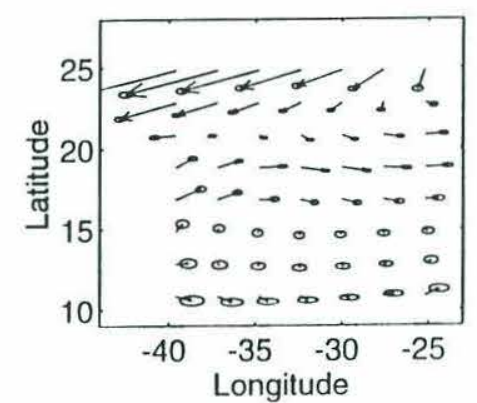

$232.595 \mathrm{~m}$

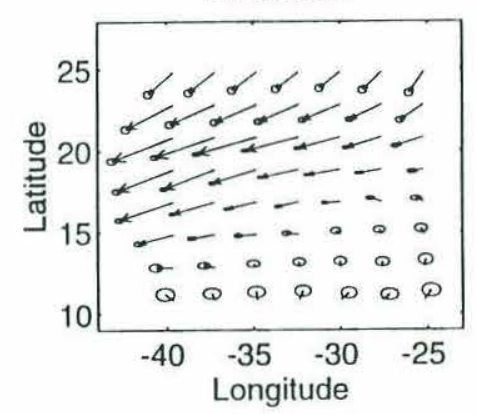

$462.505 \mathrm{~m}$

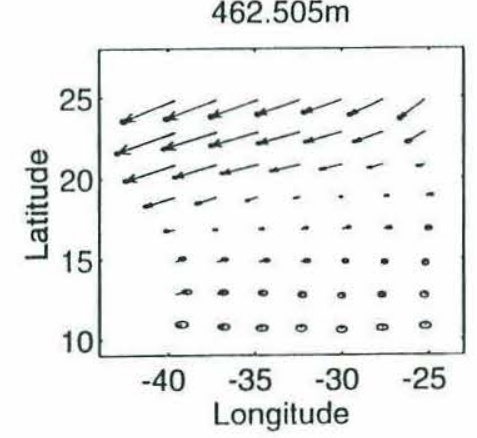

$900.89 \mathrm{~m}$

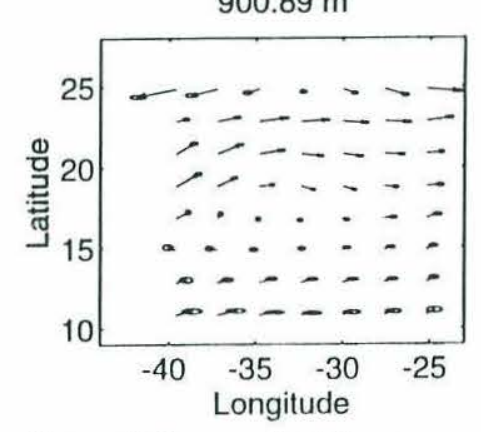

Fig. 4.20. Estimated horizontal circulations and their errors from the spatially smoothed timemean EGCM hydrographic data in the coarse grid resolution. Vector scales are the same as in Fig. 4.19. 
Estimated horizontal circulations with error ellipses. $2.4 \times 2.0$ degrees resolution, $\mathrm{NI}=13$

$1125.04 \mathrm{~m}$

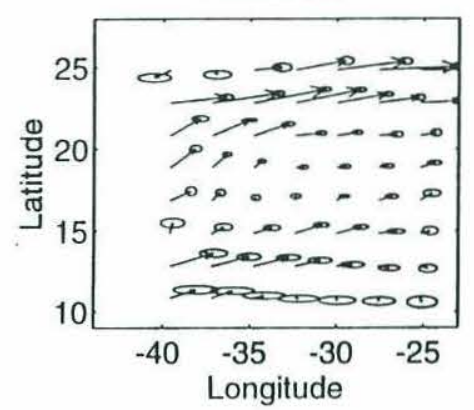

$1875.04 \mathrm{~m}$

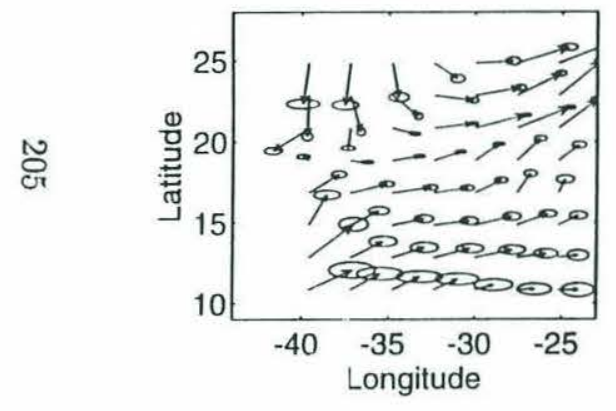

$1375.04 \mathrm{~m}$
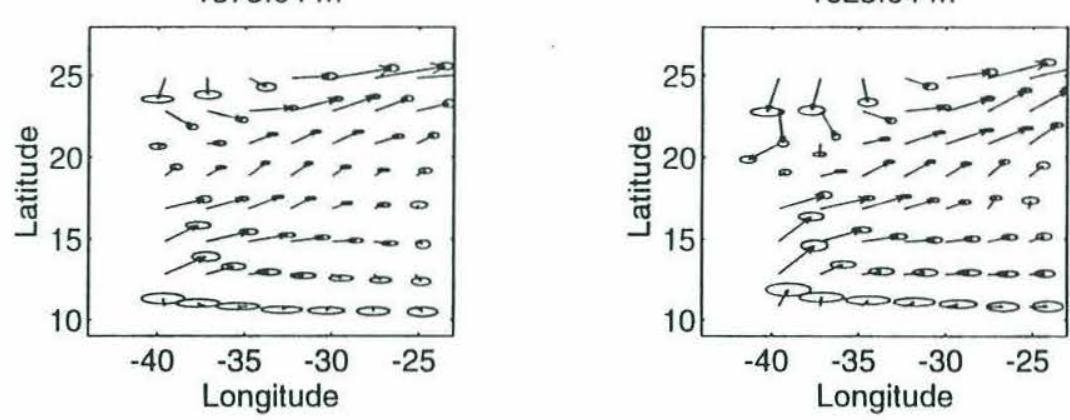

Fig. 4.20 (cont.) 
w (10 $6 \mathrm{~cm} / \mathrm{s}$ ) from GCM. $2.4 \times 2.0$ degrees smoothed. solid:positive:dashed:negative
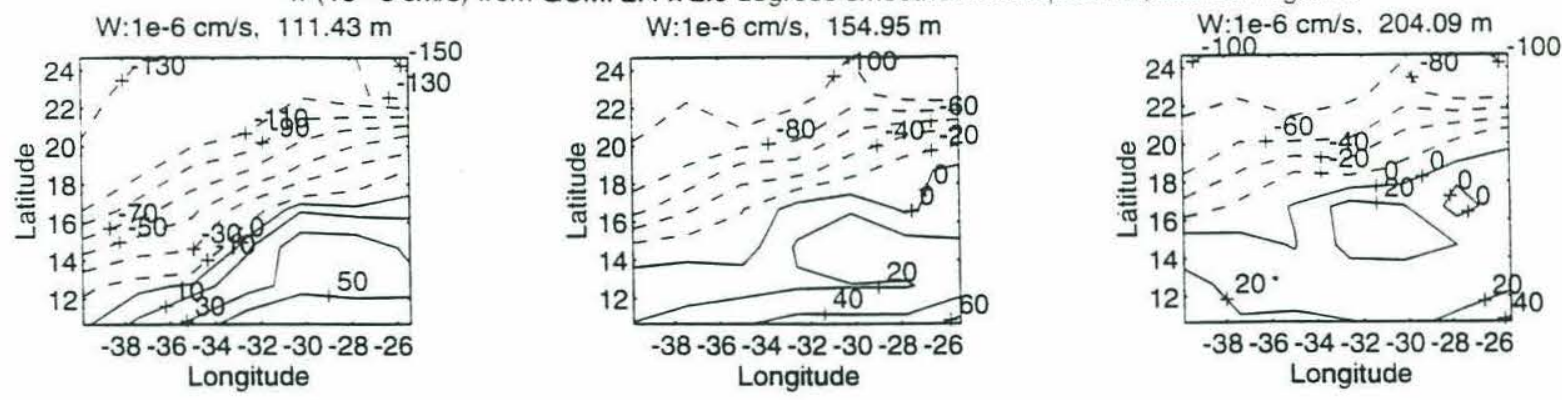

Statistical errors (95\% contidence) of horizontally smoothed w $(10 \sim 6 \mathrm{~cm} / \mathrm{s})$ trom GCM. $\mathrm{r}=6-\mathrm{pt}$.
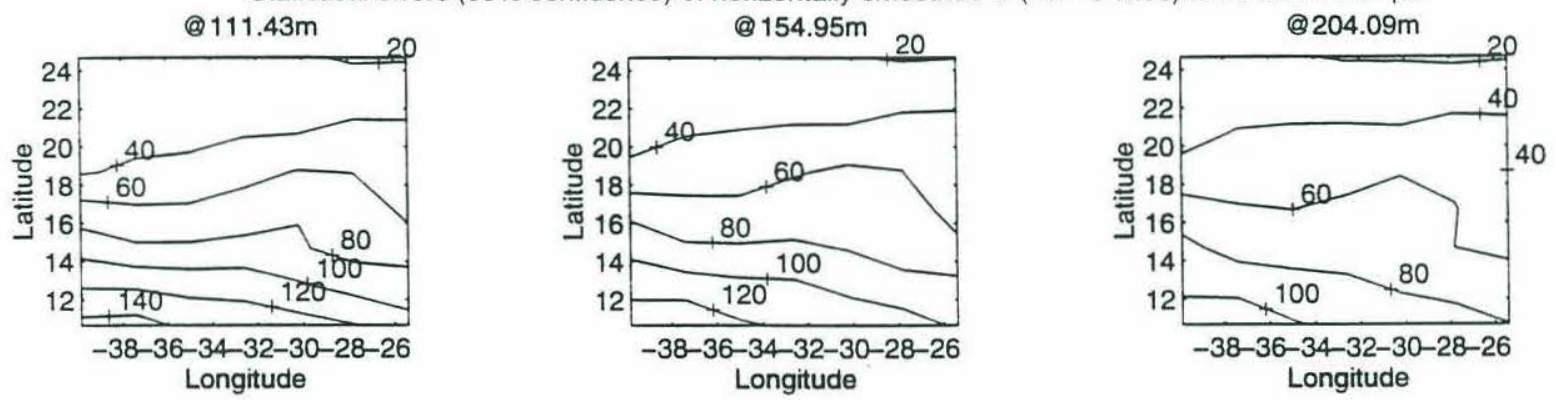

w (10 $6 \mathrm{~cm} / \mathrm{s}$ ) from GCM. $2.4 \times 2.0$ degrees smoothed. solid:positive;dashed:negative
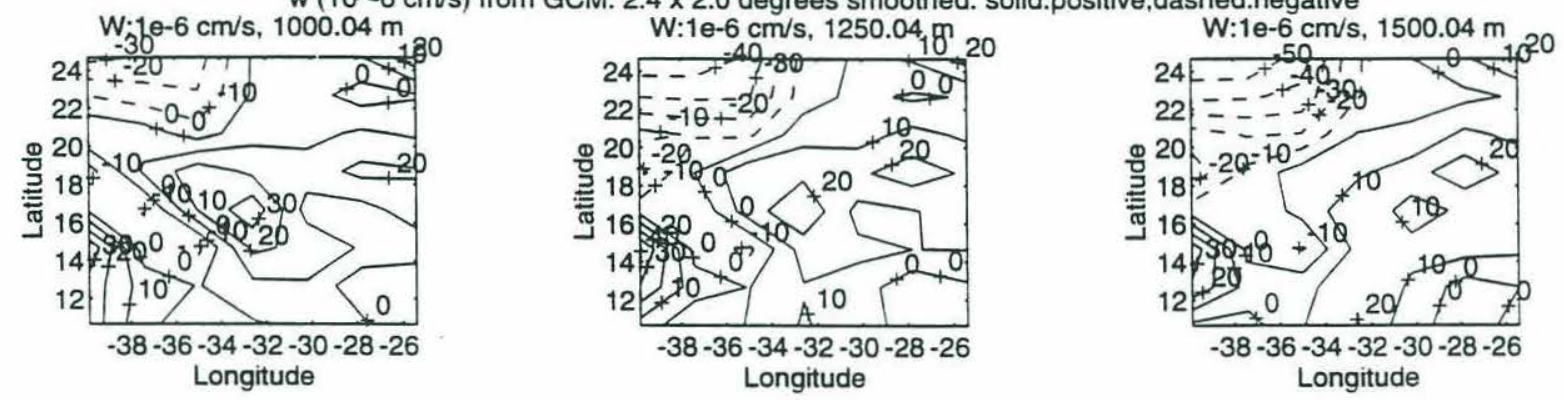

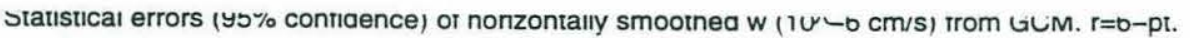
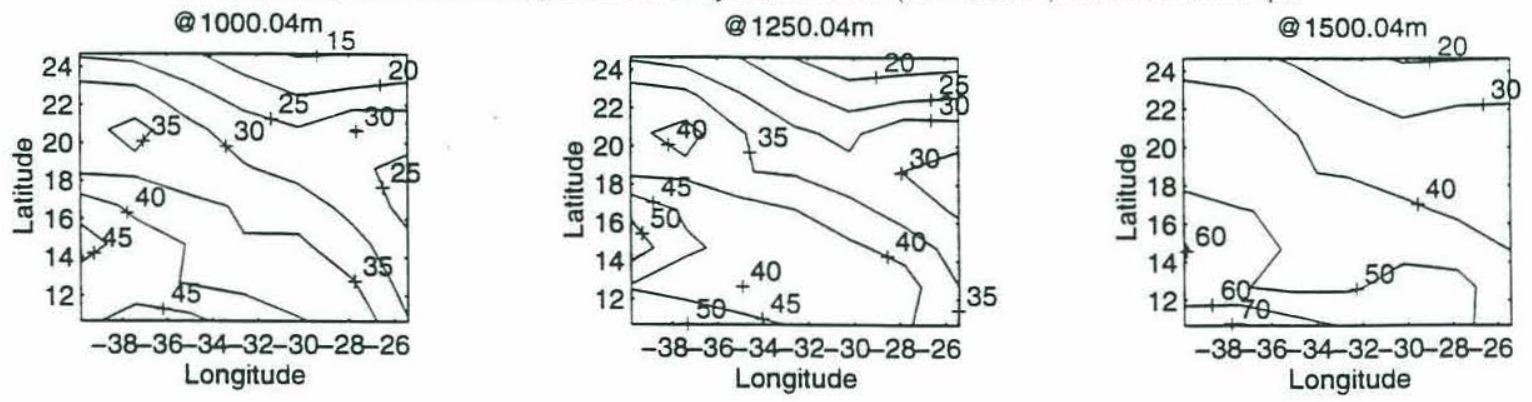

Fig. 4.21. Spatially smoothed time-mean EGCM vertical velocities and their statistical errors $\left(10^{-6} \mathrm{~cm} / \mathrm{s}\right)$. 
Estimated $\mathrm{w}$ ( 10 r $6 \mathrm{~cm} / \mathrm{s}$ ) from inverse. 2.4 by 2.0 degrees Resolution; $\mathrm{Nl}=13$. solid:positive:dashed:negative
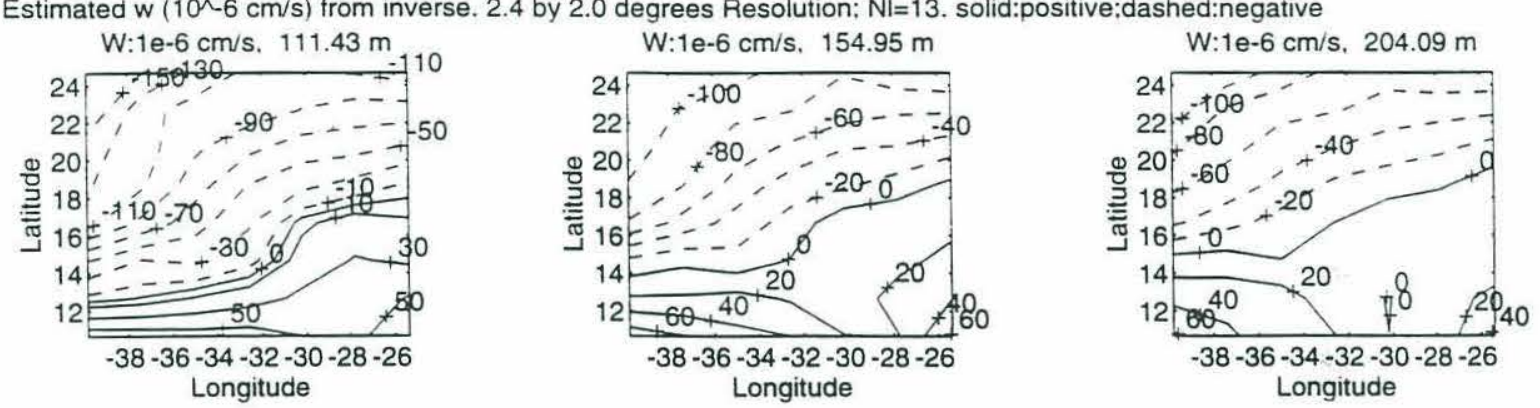

Estimated errors of $\mathrm{w}(10 \sim 6 \mathrm{~cm} / \mathrm{s})$ from inverse. $2.4 \times 2.0$ degrees resolution; $\mathrm{Nl}=13$.
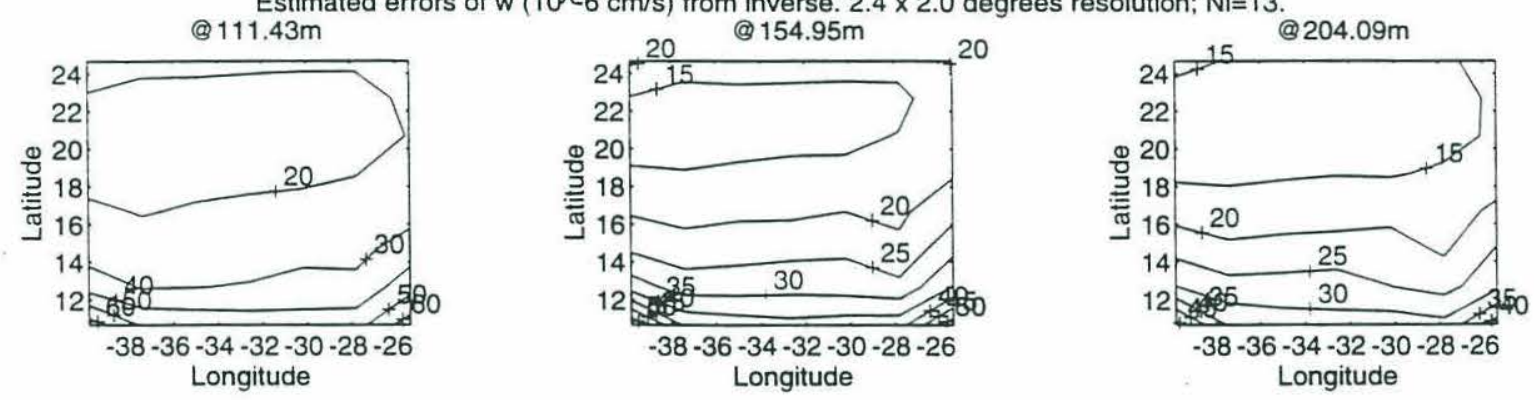

Estimated w ( $10 \sim 6 \mathrm{~cm} / \mathrm{s}$ ) from inverse. 2.4 by 2.0 degrees Resolution; $\mathrm{Nl}=13$. solid:positive;dashed:negative
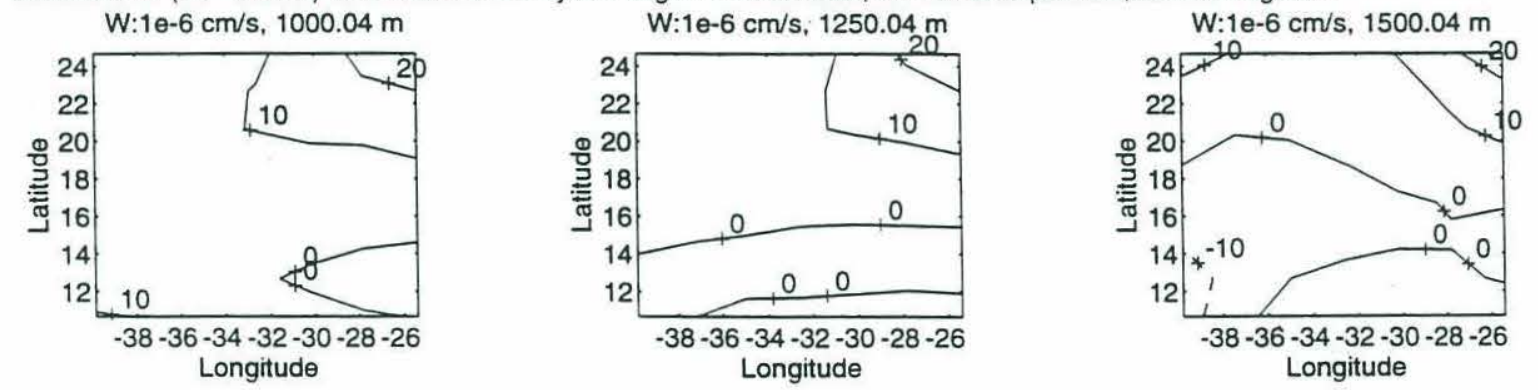

Estimated errors of $\mathrm{w}(10 \mathrm{r} 6 \mathrm{~cm} / \mathrm{s}$ ) from inverse. $2.4 \times 2.0$ degrees resolution; $\mathrm{Nl}=13$.
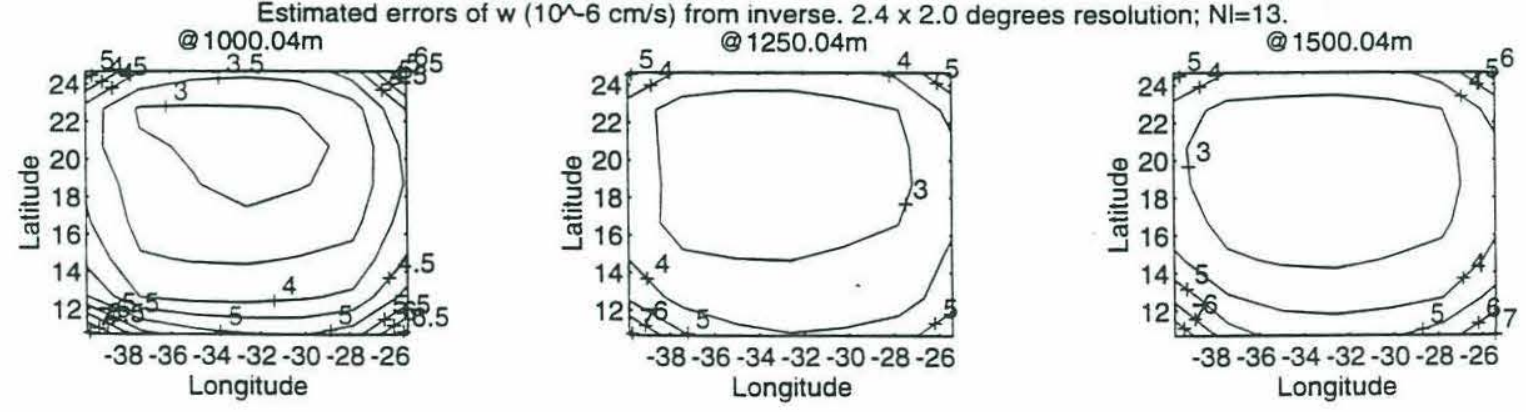

Fig. 4.22. Same as Fig. 4.20 but for vertical velocity $\left(10^{-6} \mathrm{~cm} / \mathrm{s}\right)$. 


\section{The "Diffusion" Coefficients}

\section{a). Horizontal Diffusion Coefficients}

The spatially smoothed zonal (with subscript $x$ ) and meridional (with subscript $y$ ) eddy diffusion coefficients for heat (with superscript T) $A_{x}^{T}$ and $A_{y}^{T}$ computed from the spatially smoothed zonal and meridional eddy heat fluxes of the EGCM ocean are shown in Fig. 4.23 and Fig. 4.24, along with their estimated statistical errors. In this larger domain and at shallow depths (e.g., at $133 \mathrm{~m}, 179 \mathrm{~m}$ and $232 \mathrm{~m}$ of the upper panel in Figs.4.23 and 4.24), the eddy diffusion coefficients for heat in the zonal and meridional directions have the same magnitude (of order $\left.1.0 \times 10^{7} \mathrm{~cm}^{2} / \mathrm{s}\right)$ and there is no significant difference between them. On the other hand, at great depths (e.g., at $1125 \mathrm{~m}, 1375 \mathrm{~m}$ and $1625 \mathrm{~m}$ ), the magnitudes of the zonal diffusion coefficients for heat are larger than those of the meridional ones, and the differences are marginally significant. At these depths, the horizontal diffusivity is marginally anisotropic.

The spatially smoothed zonal diffusion coefficients for salt of the EGCM ocean are shown in Fig. 4.25. Comparison of this figure with Fig. 4.23 shows that the diffusion coefficients for heat and salt are very similar in both distribution patterns and values. In fact, they are indistinguishable within the statistical errors.

As in the fine grid resolution case in Section 4.3, the horizontal diffusion coefficients in the inverse model are taken as isotropic and the same for heat and salt, and they were parameterized as the third-order discrete Tchebychev polynomial functions. The estimated values by the inverse model from the spatially smoothed time-mean hydrographic data of the EGCM ocean are shown in Fig. 4.26. Comparisons of this figure with Figs. 4.23, 4.24 and 4.25 show that at the upper levels, 
the values of the inverse estimations are in the same order as those of the direction computations (from the eddy fluxes). Although they are different in spatial patterns and point-wise values, the area-averaged values are similar. However, at great depths, the inverse model estimated values are very different from those of the direct computations: the estimated values are much smaller (at least one order) than those computed from the eddy heat and salt fluxes. Within the statistical errors, the differences between inverse model estimates and the direct computations are not obvious.

\section{b).Vertical Diffusion Coefficients.}

Shown in Fig. 4.27 and Fig. 4.28 are the spatially smoothed vertical eddy diffusion coefficients for heat and salt of the EGCM ocean. Comparisons of these two figures show that, although the vertical eddy diffusion coefficient for heat and salt have similar spatial distribution patterns (except at $1250 \mathrm{~m}$ in these figures), the values are not the same. Generally, the absolute values (as the vertical eddy diffusion coefficients are mostly negative) of the vertical eddy diffusion coefficients for salt are larger than those for heat. But their statistical errors are in the same magnitudes as the diffusion coefficients themselves, and thus the diffusion coefficients are not

significantly different from zero most of the time, and the differences between $K^{T}$ and $K^{S}$ are not significant.

Like the horizontal "diffusion" coefficients, the vertical "diffusion" coefficients in the inverse model were also taken the same for heat and salt, and they were parameterized as the third-order discrete Tchebychev polynomial functions, whose coefficients vary from depth to depth. The inverse model solutions for the 
vertical diffusion coefficients are shown in Fig. 4.29. Comparisons of this figure with Fig. 4.27 and Fig. 4.28 show that, first of all, the inverse model results for the vertical diffusion coefficients have similar spatial distribution patterns to those of the spatially smoothed vertical diffusion coefficients directly calculated from the vertical eddy fluxes of the GCM ocean. For example, the values are mostly negative in both the inverse model solutions and the direct computations. At $111 \mathrm{~m}$, the inverse model solutions are consistent with the directly calculated vertical diffusion coefficients for heat within the errors bars. At $204 \mathrm{~m}$, the estimated values are consistent with those of the directly computed vertical eddy diffusion coefficients for both heat and salt. However, at $1500 \mathrm{~m}$, the inverse model estimated values are marginally consistent with the values of the direct computations for salt, but the negative values are significantly larger than those of the direct computations for heat. 
Diffusivity ATx ( $\left.10 \wedge 7 \mathrm{~cm}^{\wedge} 2 / \mathrm{s}\right): \mathrm{r}=2.4 \times 2.0$ degrees resolution smoothed field: $\mathrm{Nl}=13$.
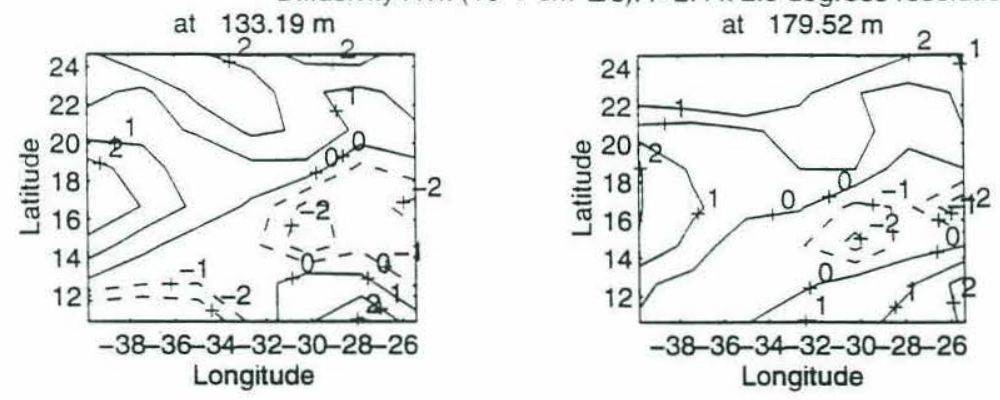

at $232.595 \mathrm{~m}$

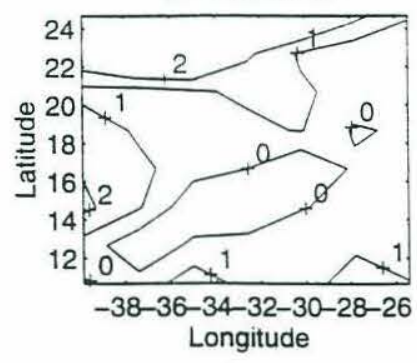

errors (95\% confidence) of ATx (10^7 $\left.\mathrm{cm}^{\wedge} 2 / \mathrm{s}\right) . \mathrm{r}=2.4 \times 2.0$ degrees resolution smoothed; $\mathrm{Nl}=13$.
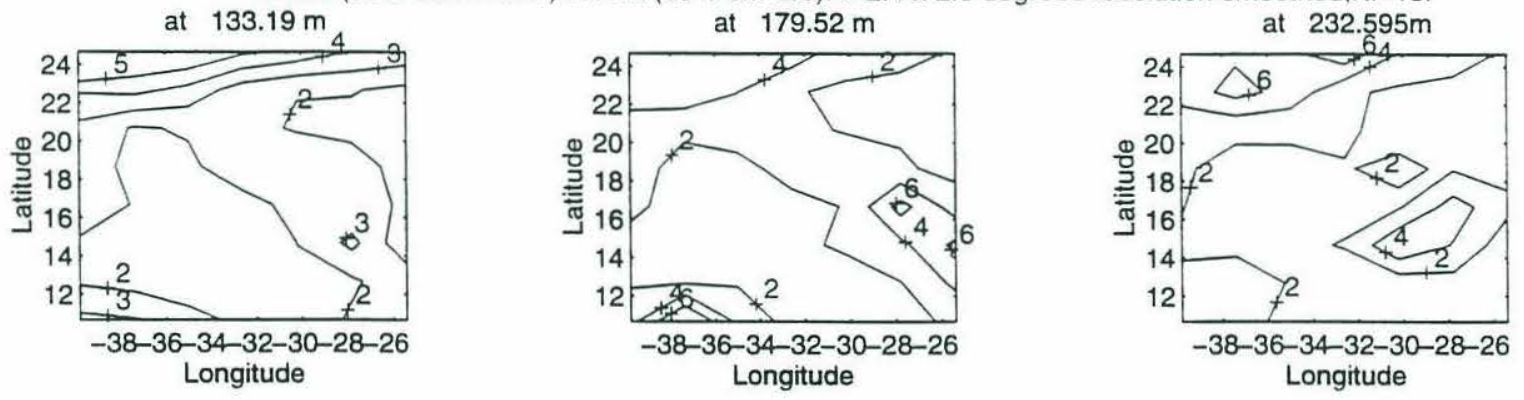

Diffusivity ATx ( $10 \wedge 7 \mathrm{~cm} / 2 / \mathrm{s}): \mathrm{r}=2.4 \times 2.0$ degrees resolution smoothed field: $\mathrm{Nl}=13$.
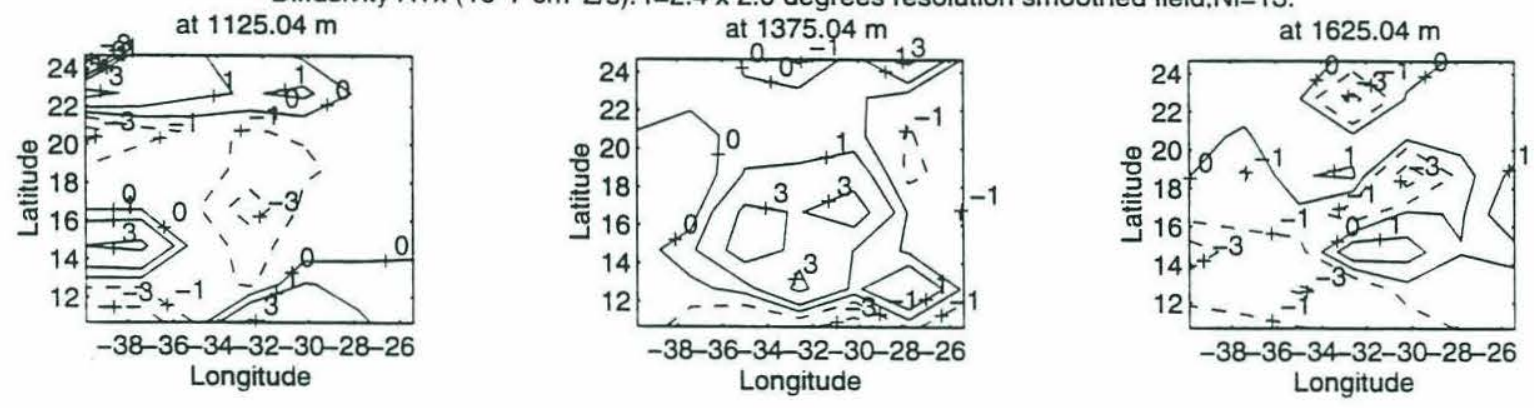

errors ( $95 \%$ confidence) of $\mathrm{ATX}\left(10 \wedge 7 \mathrm{~cm}^{\wedge} 2 / \mathrm{s}\right) . \mathrm{r}=2.4 \times 2.0$ degrees resolution smoothed: $\mathrm{Nl}=13$.
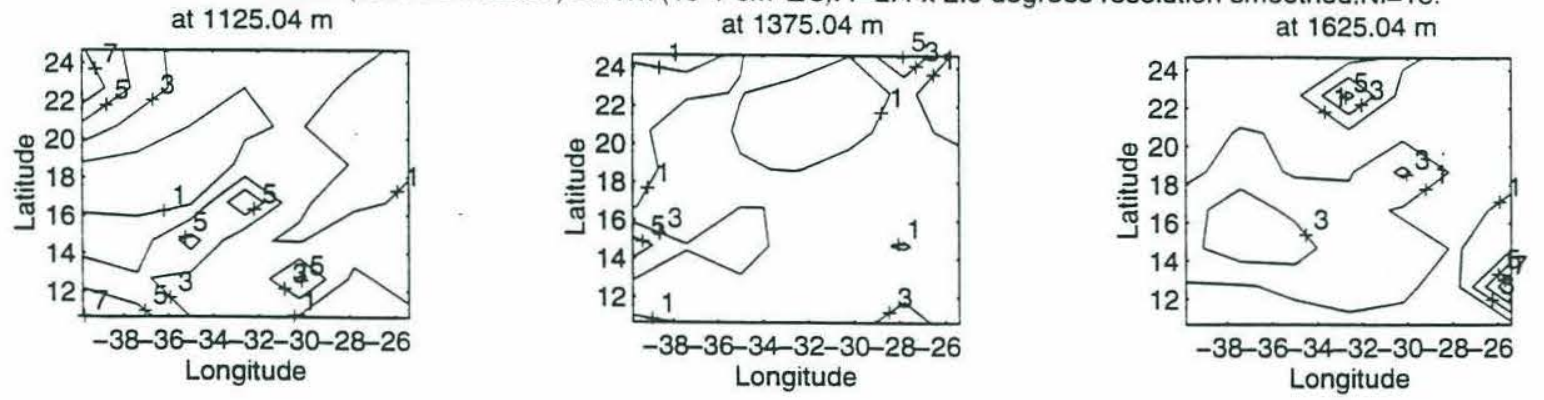

Fig. 4.23. Horizontal eddy diffusion coefficients and their statistical errors $\left(10^{7} \mathrm{~cm}^{2} / \mathrm{s}\right)$ computed from the smoothed EGCM eddy fluxes for heat in zonal direction. 
Diffusivity ATy $\left(10 \wedge 7 \mathrm{~cm}^{\wedge} 2 / \mathrm{s}\right): r=2.4 \times 2.0$ degrees resolution smoothed field: $\mathrm{Nl}=13$.
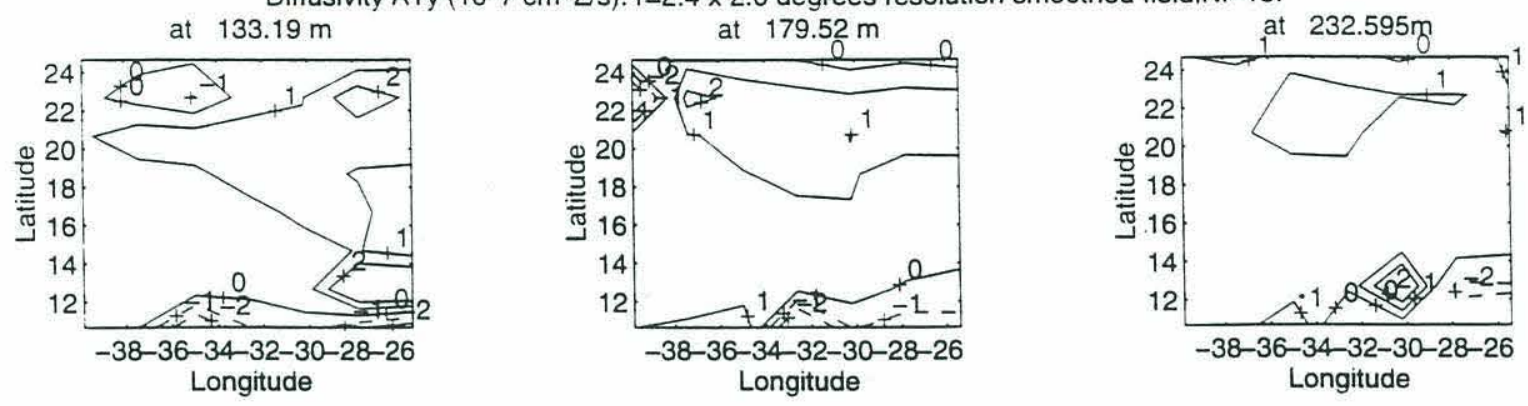

errors (95\% confidence) of ATy ( $\left.10 \wedge 7 \mathrm{~cm}^{\wedge} 2 / \mathrm{s}\right) . \mathrm{r}=2.4 \times 2.0$ degrees resolution smoothed: $\mathrm{Nl}=13$.
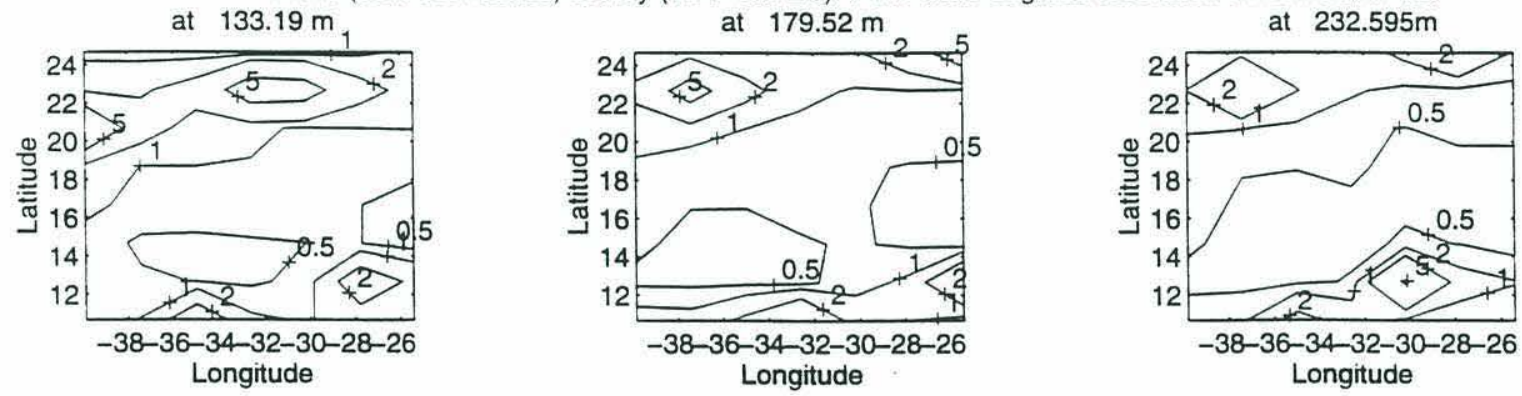

Diftusivity ATy $(10 \wedge 7 \mathrm{~cm} \wedge 2 / \mathrm{s}): \mathrm{r}=2.4 \times 2.0$ degrees resolution smoothed field; $\mathrm{Nl}=13$.
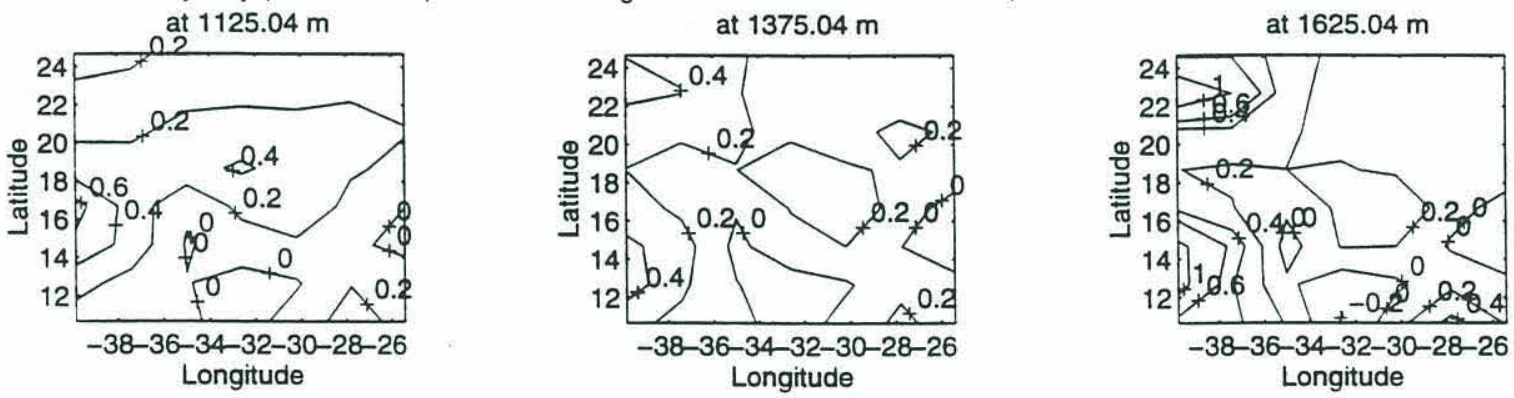

errors ( $95 \%$ contidence) of ATy $\left(10 \wedge 7 \mathrm{~cm}^{\wedge} 2 / \mathrm{s}\right)$. $\mathrm{r}=2.4 \times 2.0$ degrees resolution smoothed; $\mathrm{Nl}=13$.
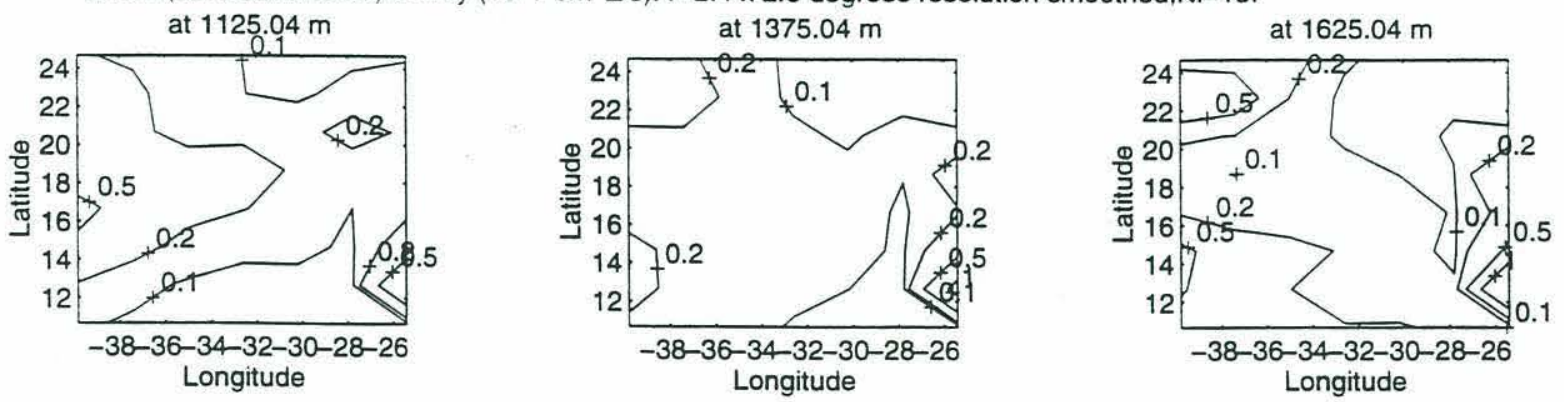

Fig. 4.24. Same as Fig. 4.23 but in meridional direction. 
Diffusivity ASx $\left(10 \wedge 7 \mathrm{~cm}^{\wedge} 2 / \mathrm{s}\right): \mathrm{r}=2.4 \times 2.0$ degrees resolution smoothed field: $\mathrm{Nl}=13$.
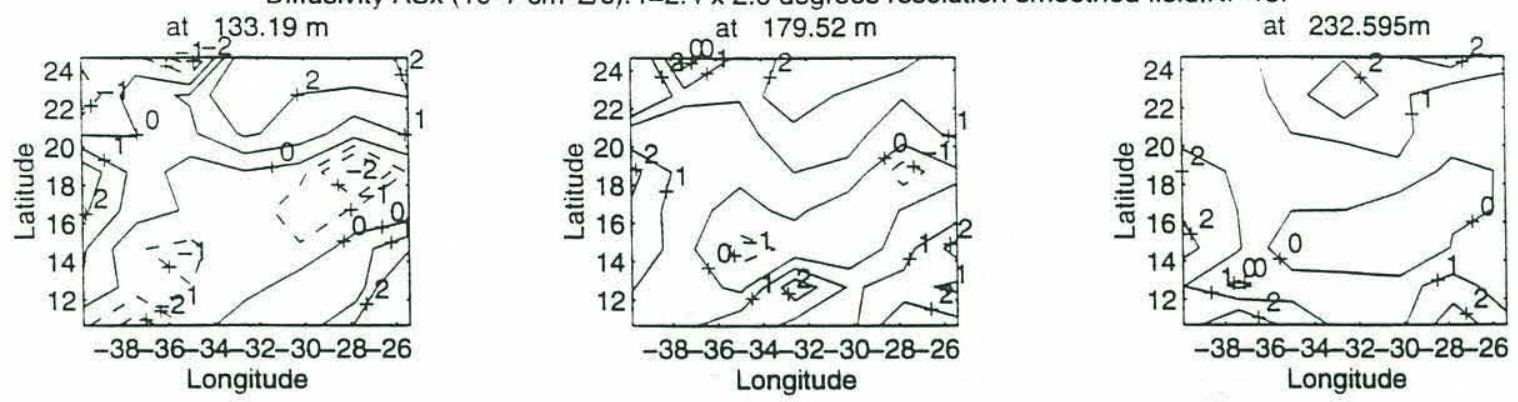

errors ( $95 \%$ confidence) of $\mathrm{ASx}(10 \wedge 7 \mathrm{~cm} \wedge 2 / \mathrm{s}) . \mathrm{r}=2.4 \times 2.0$ degrees resolution smoothed; $\mathrm{Nl}=13$.

at $133.19 \mathrm{~m}$

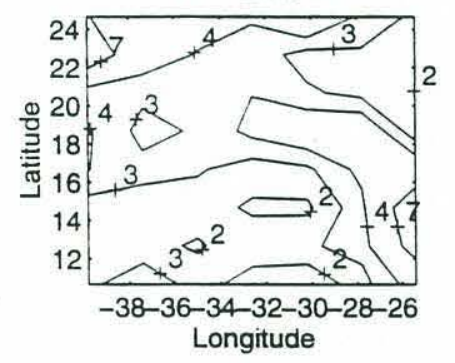

at $179.52 \mathrm{~m}$

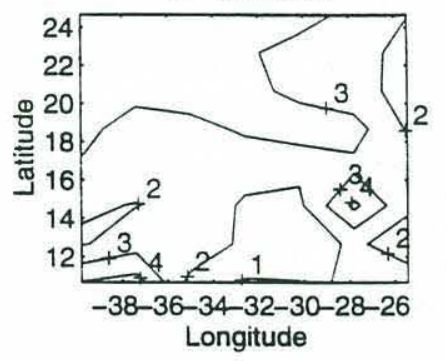

at $232.595 \mathrm{~m}$

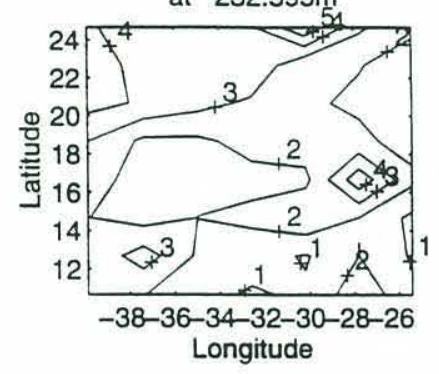

Diffusivity ASx (10^7 $\left.\mathrm{cm}^{\wedge} 2 / \mathrm{s}\right): \mathrm{r}=2.4 \times 2.0$ degrees resolution smoothed field; $\mathrm{Nl}=13$.
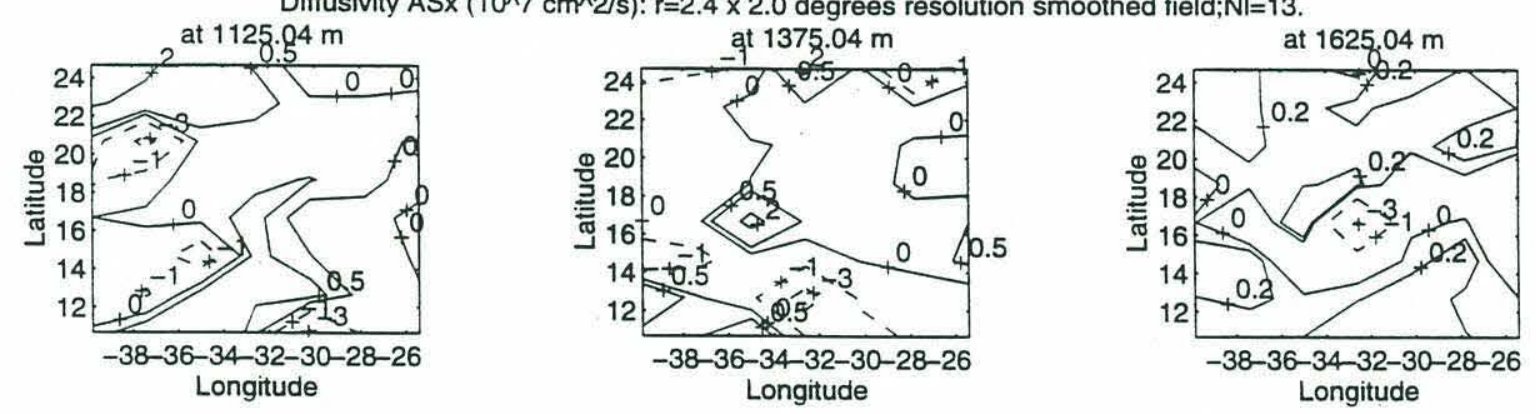

errors (95\% confidence) of $\mathrm{ASx}\left(10^{\wedge} 7 \mathrm{~cm}^{\wedge} 2 / \mathrm{s}\right) . \mathrm{r}=2.4 \times 2.0$ degrees resolution sinoothed: $\mathrm{Nl}=13$.
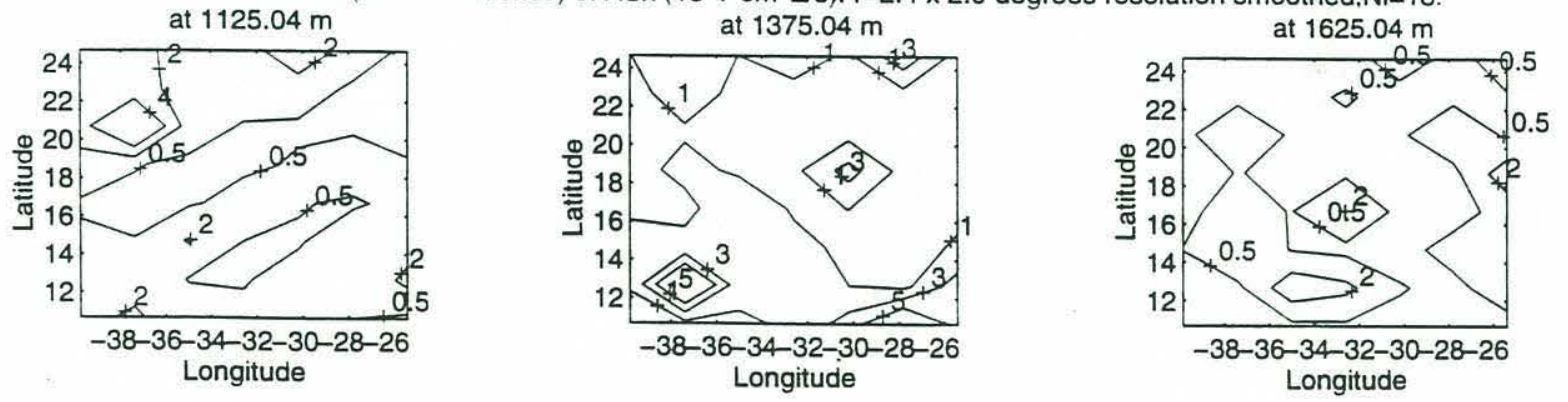

Fig. 4.25. Same as Fig. 4.23 but for salt fluxes. 

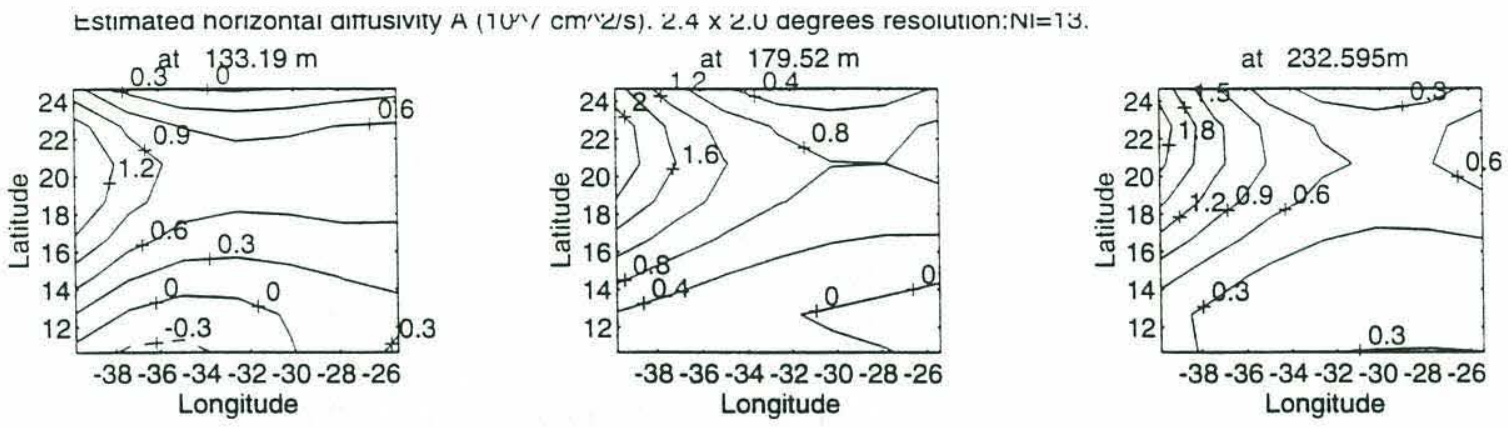

Estimated errors for horizontal diffusivity $\mathrm{A}\left(10 \wedge 7 \mathrm{~cm}^{\wedge} 2 / \mathrm{s}\right) .2 .4 \times 2.0$ degree resolution, $\mathrm{Nl}=13$
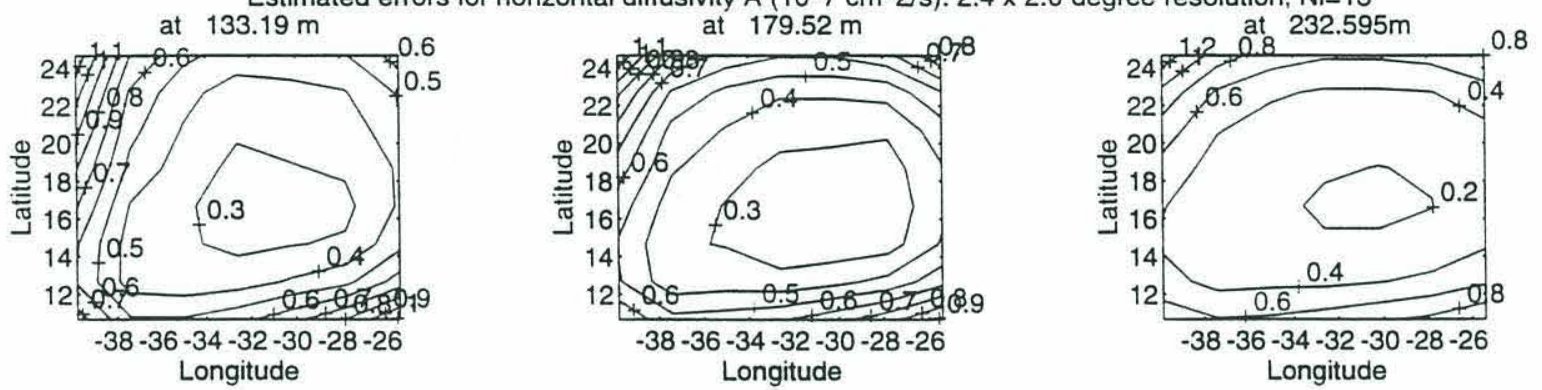

Estimated horizontal diffusivity $\mathrm{A}(10 \wedge 7 \mathrm{~cm} \wedge 2 / \mathrm{s}) .2 .4 \times 2.0$ degrees resolution; $\mathrm{Nl}=13$.
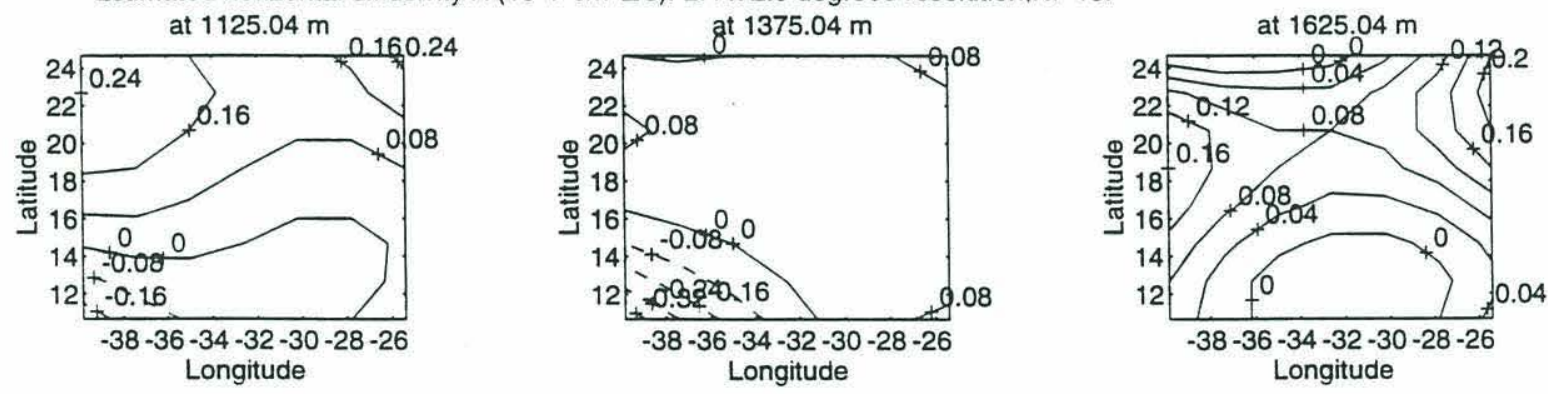

Estimated errors for horizontal diffusivity $\mathrm{A}(10 \wedge 7 \mathrm{~cm} \wedge 2 / \mathrm{s}) .2 .4 \times 2.0$ degree resolution, $\mathrm{Nl}=13$
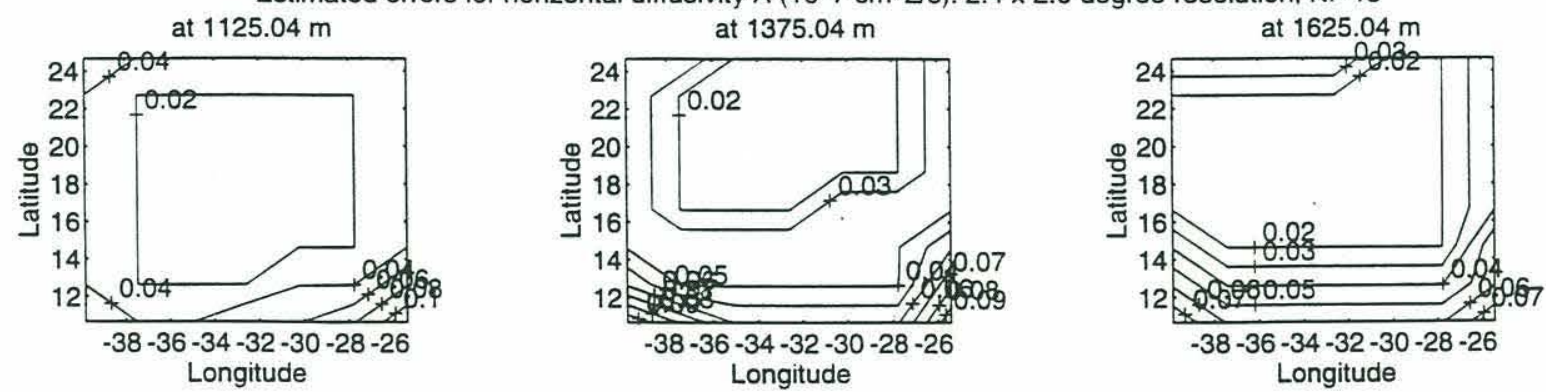

Fig. 4.26. Estimated horizontal diffusion coefficients $\left(10^{7} \mathrm{~cm}^{2} / \mathrm{s}\right)$ and their errors from the smoothed time-mean EGCM hydrographic data in the coarse grid resolution. 

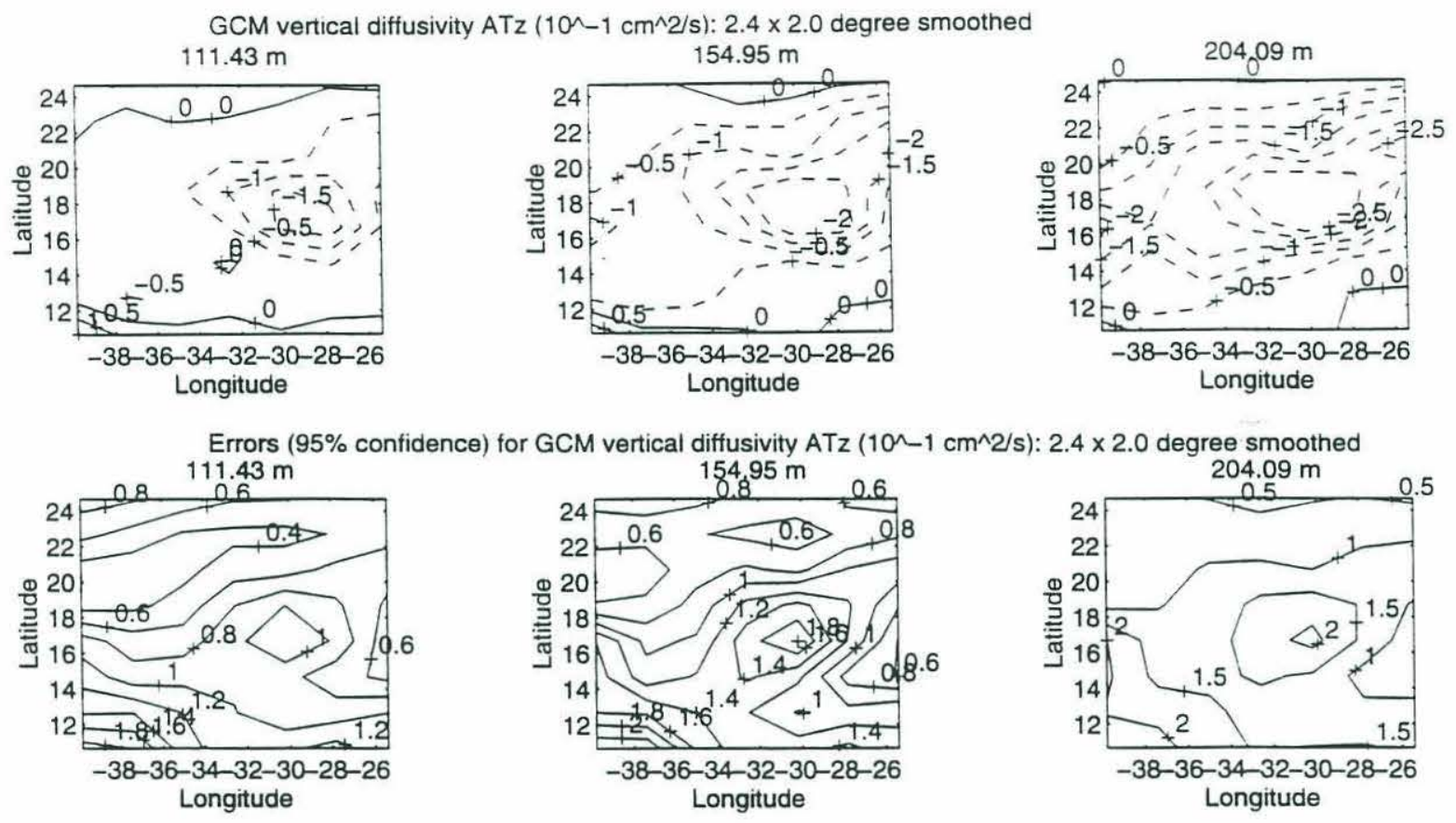

GCM vertical diffusivity ATz (10 $\left.-1 \mathrm{~cm}^{\wedge} 2 / \mathrm{s}\right): 2.4 \times 2.0$ degree smoothed
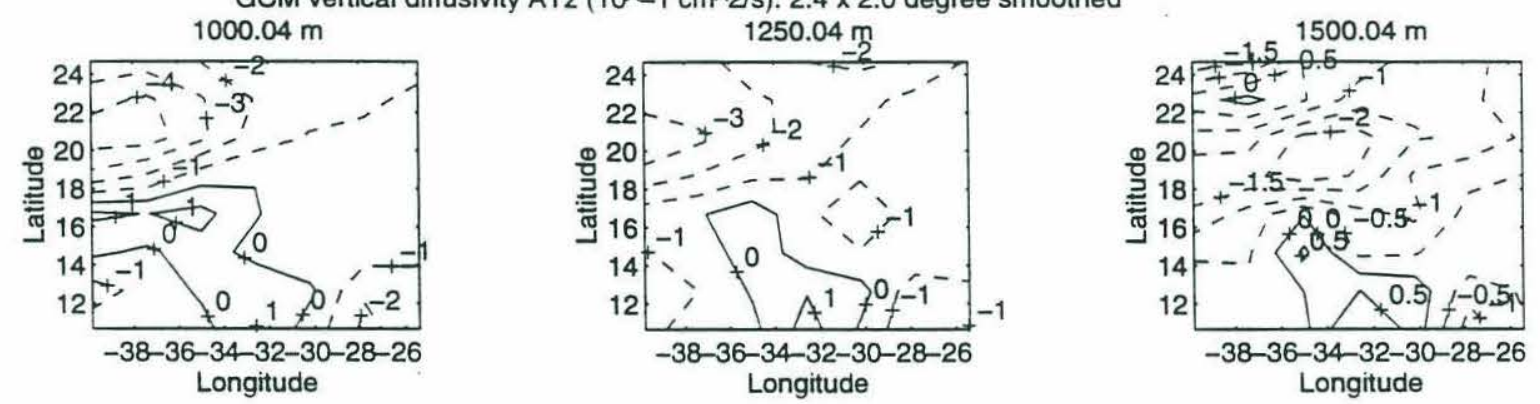

Errors (95\% confidence) for GCM vertical diffusivity ATz $\left(10 \sim-1 \mathrm{~cm}^{\wedge} 2 / \mathrm{s}\right): 2.4 \times 2.0$ degree smoothed
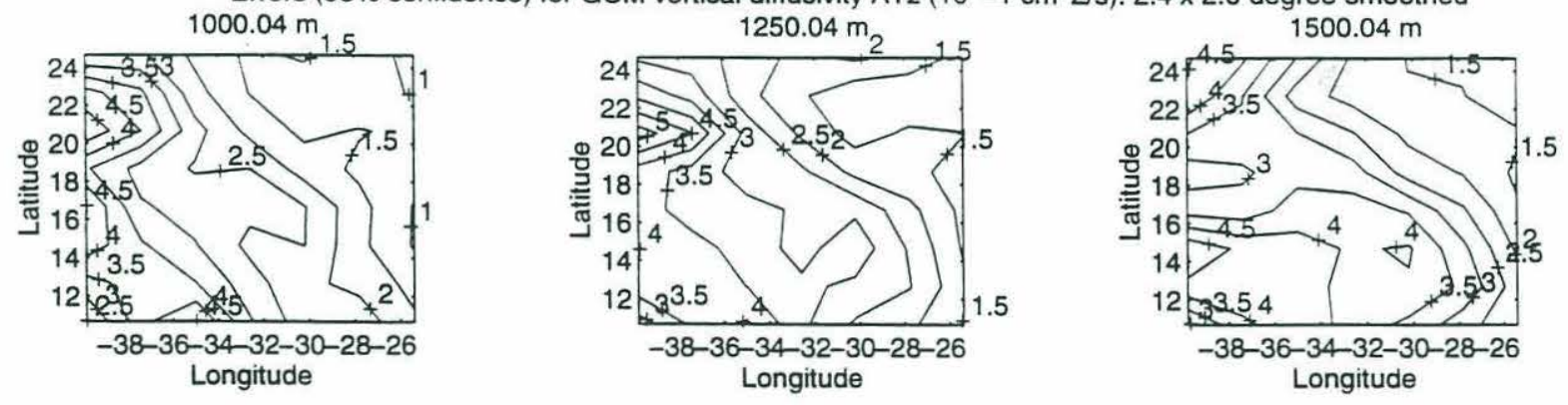

Fig. 4.27. Vertical eddy diffusion coefficients and their statistical errors $\left(\mathrm{cm}^{2} / \mathrm{s}\right)$ computed from the smoothed EGCM vertical eddy fluxes for heat. 

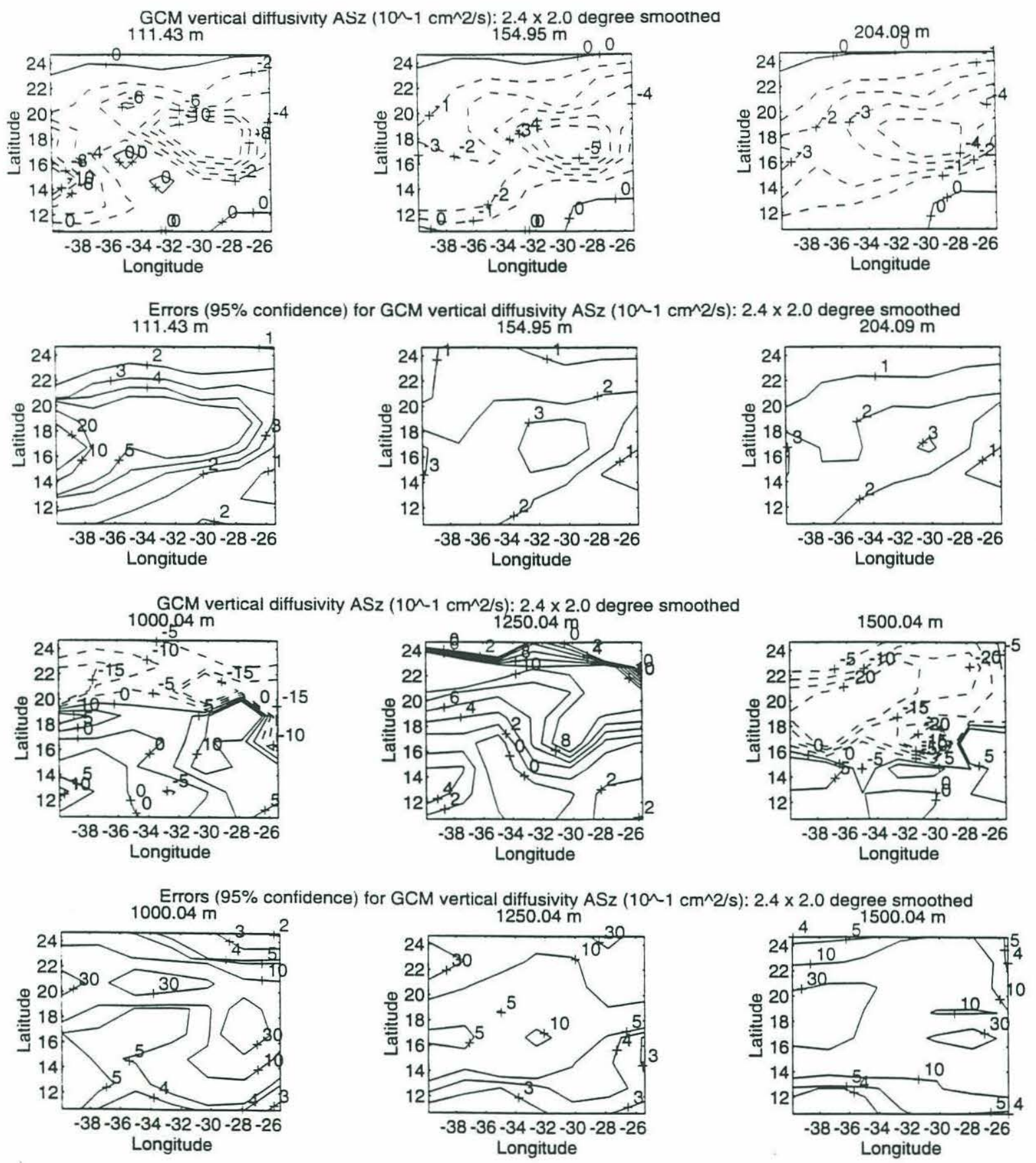

Fig. 4.28. Same as Fig. 4.27 but for salt. 

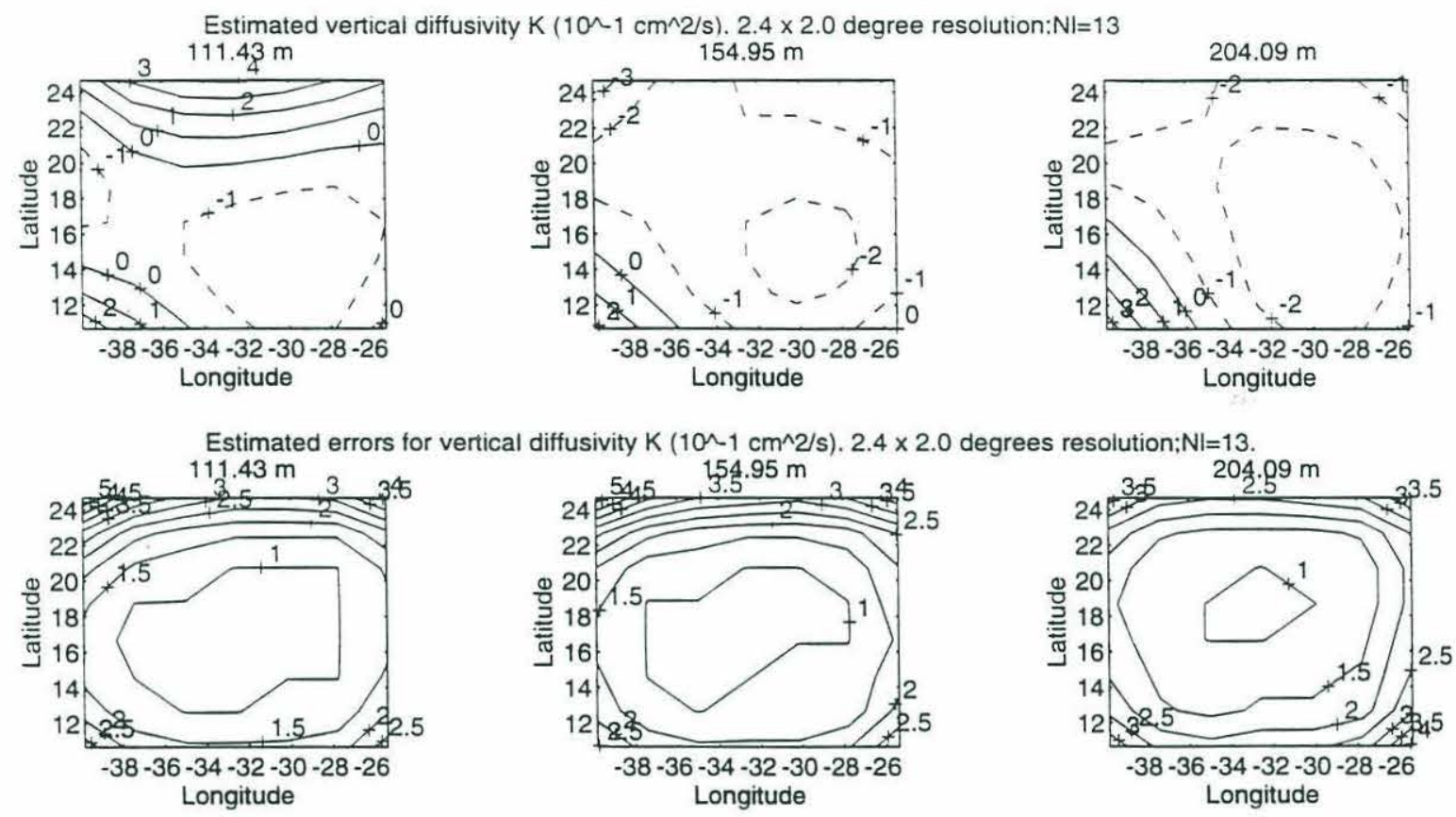

Estimated vertical diffusivity $\mathrm{K}\left(10 \wedge-1 \mathrm{~cm}^{\wedge} 2 / \mathrm{s}\right) .2 .4 \times 2.0$ degree resolution; $\mathrm{Nl}=13$
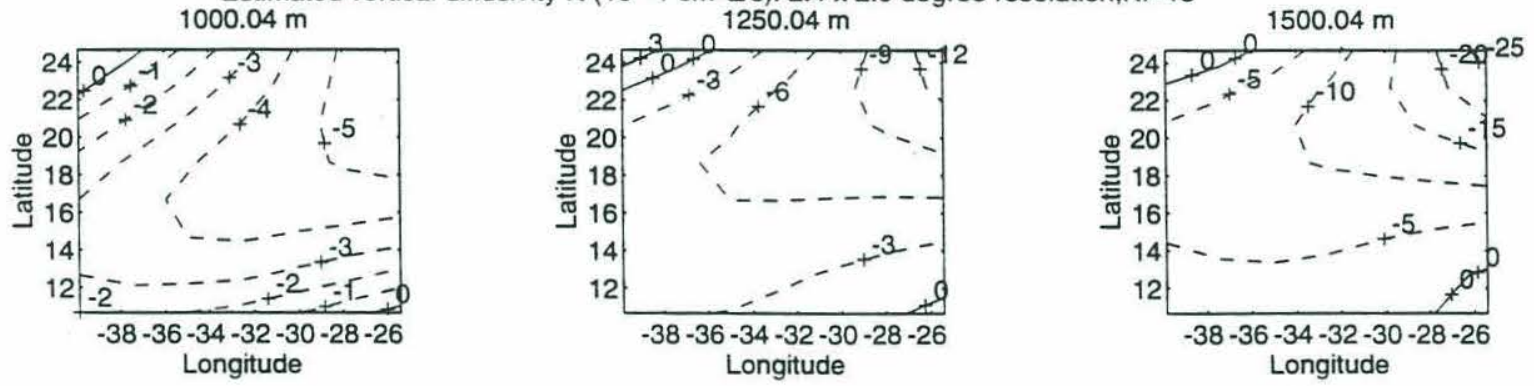

Estimated errors for vertical diffusivity $\mathrm{K}\left(10 \sim 1 \mathrm{~cm}^{\wedge} 2 / \mathrm{s}\right) .2 .4 \times 2.0$ degrees resolution; $\mathrm{Nl}=13$.
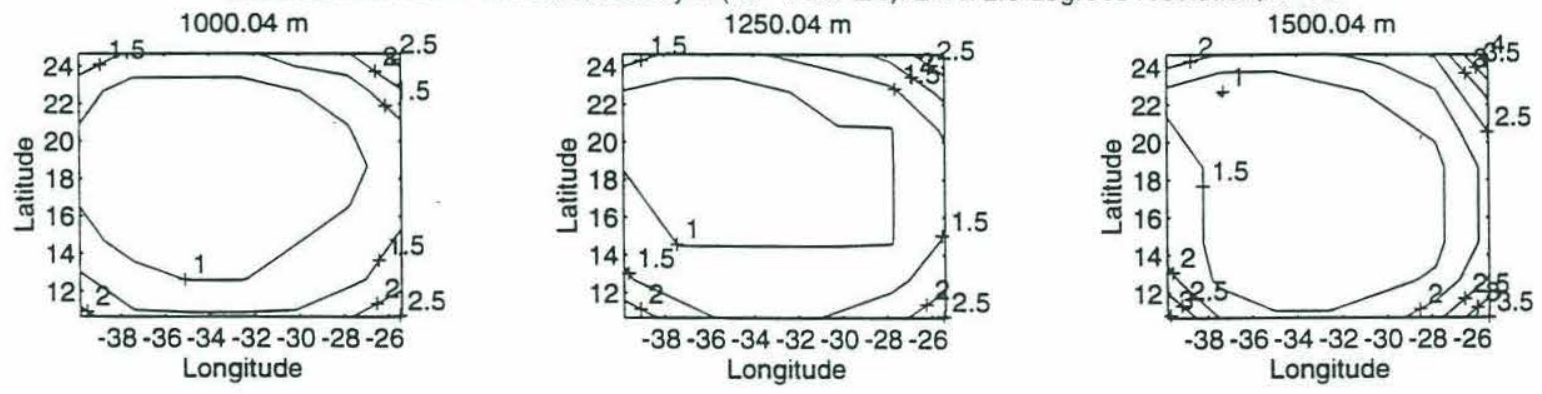

Fig. 4.29. Same as Fig. 4.26 but for vertical diffusion coefficients $\left(\mathrm{cm}^{2} / \mathrm{s}\right)$. 


\subsubsection{Sensitivity of the Inverse Model Results on the Ver- tical Level (Layer) Number}

In section 4.3.1, it was pointed out that the inverse model results are relatively insensitive to the number of the horizontal grid points but are more sensitive to the number of the vertical levels (layers). One example is reported in this section.

The results discussed in the previous section came from the application of the inverse model to the spatially smoothed time-mean hydrographic data of the numerical GCM ocean in a domain consisting of grid points of $9 \times 10 \times 13$ in the $x, y$, and $z$ direction. Vertically there are thirteen levels $(\mathrm{Nl}=13)$ on which the dynamic equation, continuity equation, and conservation equations for heat and salt were formulated. In this section, using the same horizontal and vertical grid resolution of the previous section, the inverse model is applied in the same horizontal domain but only to the upper seven vertical levels $(\mathrm{Nl}=7)$, with the vertical depth extends from $179 \mathrm{~m}$ to $721 \mathrm{~m}$.

As reported before, the 13-level run produces the horizontal circulations (Fig. 4.20) which are consistent with the spatially smoothed time-mean horizontal circulations of the numerical GCM ocean (Fig. 4.19) on all the eight levels above $722 \mathrm{~m}$ (including the level at the depth of $721 \mathrm{~m}$ ). However, the results from the 7-level run are not as good, as shown in Fig. 4.30. Comparisons of this figure with Fig. 4.19 shows that below $462 \mathrm{~m}$, the inversions are significantly different from the spatially smoothed time means of the numerical GCM ocean in both flow patterns

and values. Even at the shallower depths (where the flows are stronger), there are some significant differences between the inversions and spatially smoothed time 
means, especially at the northern edge, although the large scale flow patterns are similar.

Due to the poorer estimations of the horizontal circulations, the estimated vertical velocities and "diffusion" coefficients are worse in terms of closeness to their "true" values in the spatially smoothed time-mean numerical GCM ocean. This experiment and other experiments (which demonstrated that the inverse model results are not so sensitive to the horizontal grid numbers) indicate that, if one is limited by the available computer resource, one should choose a domain which consists of more vertical levels and less horizontal grid points, instead of less vertical levels and more horizontal grid points. 
Estimated horizontal circulations with error ellipses. $2.4 \times 2.0$ degrees resolution, $\mathrm{NI}=7$ $179.52 \mathrm{~m}$
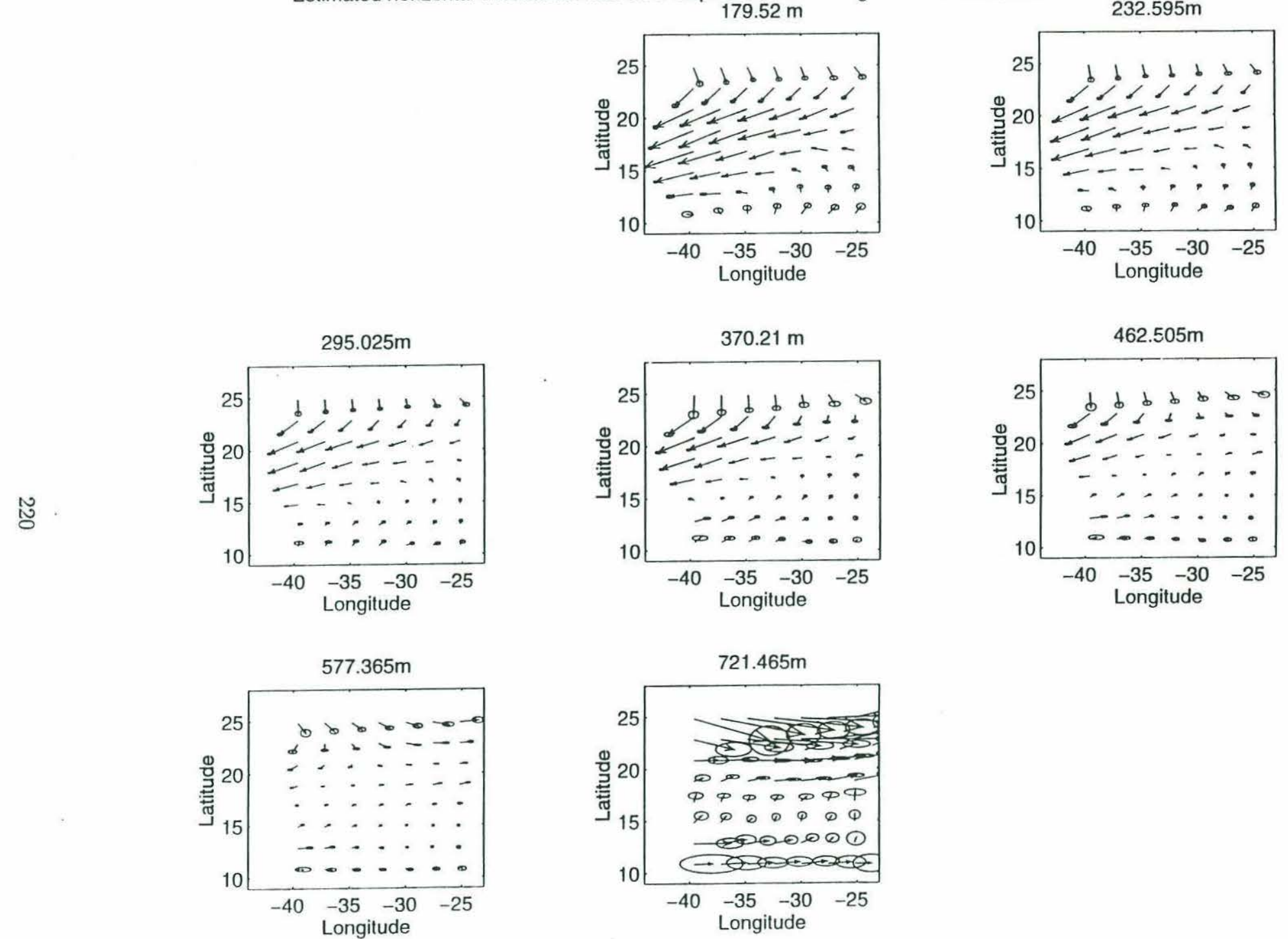

Fig. 4.30. Same as Fig. 4.20 but this run has only seven vertical layers, 


\subsubsection{Summary and Discussion}

In this section, the inverse model was applied to the spatially smoothed timemean hydrographic data of the eddy resolving GCM ocean. The main objective was to test the hypothesis that the large scale (spatially smoothed) time-mean oceanic circulations could be effectively deduced from the spatially smoothed time-mean hydrographic data by the inverse model.

The accuracy of the inverse model equations in the spatially smoothed timemean fields of the numerical GCM ocean in a coarse grid resolution was first examined. It is shown that, unlike the time-mean (unsmoothed) velocity fields which satisfy the continuity equation exactly in the fine grid resolution of the EGCM $\left(2 / 5^{\circ}\right.$ longitude by $1 / 3^{\circ}$ latitude), the spatially smoothed time-mean velocity fields do not satisfy the continuity equations exactly in the coarse grid resolution $\left(2.4^{\circ}\right.$ longitude by $2.0^{\circ}$ latitude). The imbalances (errors) are caused by the subsampling aliasing. These error terms are not important compared to the individual divergence terms in the upper layers (at shallow depths), but they are quite significant compared to the vertical velocity divergence at great depths, although they are much smaller than the individual (zonal and meridional) horizontal velocity divergence terms.

The spatially smoothed time-mean horizontal circulations are in approximate thermal wind balance, with domain-wide averaged relative imbalance (between the absolute velocity shear and the thermal wind shear) of $9 \%$. These imbalances in the coarse grid resolution are larger than those of the (unsmoothed) time-mean velocity fields in the fine grid resolution (with the averaged relative imbalance of 5\%).

The spatially smoothed time-mean temperature and salinity fields are not balanced by the terms proposed in the steady state conservation equations for heat 
and salt of the inverse model (the horizontal and vertical advection terms and eddy diffusion terms (eddy fluxes)). In addition to the errors linked to the spatial smoothing and larger grid spacing mentioned above, there are also some physical terms which are not included in the inverse model. In this coarse grid resolution scheme, although the biharmonic horizontal dissipation terms are greatly reduced and generally insignificant, the temporal variation terms (which arose from the limited time period of the time means) are still important. In the upper layers, the dominant terms in the conservation equations are the horizontal and vertical advection terms. However, in the deep layers, the vertical advection terms are reduced and are not among the dominant terms.

The inverse model estimated horizontal circulations from the spatially smoothed time-mean hydrographic data of the numerical GCM ocean using the coarse grid resolution are very similar to the spatially smoothed time-mean horizontal circulations of the numerical GCM ocean. At the eight levels above $722 \mathrm{~m}$ depth, the inverse model estimations are totally consistent with the spatially smoothed timemean GCM velocities within the statistical errors. In the deep layers, there are some significant differences between the inverse model estimations and those of the spatially smoothed GCM time means in few regions. However, the estimated horizontal circulations still have the same large scale flow patterns as those of the GCM ones, and the values are generally consistent with each other within the error ellipses.

The inverse model solutions are closer to the values of the spatially smoothed time-mean vertical velocity of the GCM ocean in this coarse grid resolution scheme than those of the fine grid resolution scheme in section 4.3. In the upper layers, the inverse estimations are consistent with those of the spatially smoothed time means in both distribution patterns and values within the statistical errors. However, 
significant differences occur in the deep layers. The poor solutions for the vertical velocity in the deep layers are associated with the small amplitude signals of the vertical advection terms in the heat and salt conservation equations (they are smaller than those of the temporal variation terms which are neglected in the inverse model equations). The differences can also be attributed to the not so good estimations of the horizontal circulations in the deep layers. 


\section{Chapter 5. CONCLUSION AND DISCUSSION}

Oceanic circulation is an important mechanism for heat and other property transport in the climate system. However, the picture of the general circulation (the large scale climatological mean circulation) in the world oceans, especially at great depth and in the ocean interior, is far from complete.

The main obstacle in studying the oceanic general circulation is that direct measurement of current in the ocean is extremely difficult. In addition to the difficulty in instrumentation, it is difficult to extract the time-mean part of the circulations from incomplete time series of current measurements. The oceans are full of meso-scale eddies, whose circulations are more energetic (e.g., Schmitz and Hogg, 1983; Tarbell et al., 1994).

On the other hand, water property fields (temperature, salinity, and other tracers) are relatively stable, and they are better defined in the world oceans from historical in-situ observations. The determination of mean oceanic circulations from distributions of water properties dates back to the beginning of physical oceanography. Although direct current measurements are now made more frequently than ever before (like the recent WOCE activities in current-meter moorings, ADCP measurements, floats and drifters), it is very likely that hydrographic data and

chemical tracers as well as biological nutrients will remain the principal source of information, together with the information from satellite measurement, for determining the general oceanic circulation in the world oceans. This is due partly to the expense of making direct measurements, partly to the time length of velocity records required for a reasonable estimate of the time-mean circulation, and partly 
to the increasing number of tracer distributions that are becoming available from large-scale surveys.

In the traditional descriptive method, property distributions of seawater are used to deduce large scale flow patterns qualitatively. On the other hand, in the dynamic method, the density field is used to compute the vertical shears of the lateral velocity quantitatively. These two ideas are combined together in the formalism of inverse models in physical oceanography. Flow fields are sought to simultaneously be consistent with property distributions in the ocean and be in thermal wind balance. The earliest inverse models were formulated for determining the velocities at the reference level, using mass (density) conservation as constraints. Mixing coefficients were incorporated into the inverse models as unknowns when conservation equations for water properties (heat, salt, oxygen and other tracers) were added to the constraints. Some models also estimated the air-sea heat fluxes (e.g., Gaspar et al, 1990).

Inverse modeling activities in oceanography have recently been intensified, aided by the oncoming observational data stream of WOCE and the advance of computer power. The data sets anticipated from WOCE (in situ observations of hydrography and tracers as well as nutrients, floats and drifters, current-meter moorings, altimetry, windstress, direct estimates of water mass fluxes across various straits and sills) will greatly reduce the uncertainty over the physical state of the ocean. The more accurate data bring great opportunity for inverse modelers to study the oceanic general circulation as well as mixing processes, and their roles in the climate system. Inverse models have been and are being developed in a variety of complexity and employ a wide range of mathematical techniques, with the aim to obtain a more objective picture of the general ocean circulation. 
Although inverse methods are self-consistent (in the sense that they can give not only the estimates of the parameter values, but also their uncertainties as well as other information on the data and solutions), direct comparison of inverse model results against reality is difficult, due to the lack of direct current measurements. Bigg (1985) and Killworth and Bigg's (1988) works raised doubts about the reliability of the inverse models they tested. They concluded that results from the beta-spiral method were very poor compared to the GCM "data". "Validation" of inverse models is still an unsolved problem, and this issue has been pursued in the present work.

The inverse model tested in this work is of finite difference type (Hogg, 1987). The assumptions used in the model are that large scale ocean circulations are in approximate geostrophic and hydrostatic balances (the dynamic constraints), and that mass, heat, and salt are approximately conserved in steady state (tracer conservation constraints). Tracers such as temperature and salinity have a small range of variation around a large average value. In order to prevent errors in the advection of this large offset from dominating the residuals and thus disguising the signals from the diffusion terms, an equation is formulated for the tracer anomaly (anomaly from a average value). The unknowns of the model are the streamfunctions for the horizontal circulations (point-wise unknowns), the vertical velocities (point-wise or polynomial functions in space), and the horizontal and vertical "diffusion" coefficients (constant or polynomial functions). Unlike the inverse models formulated for the reference-level velocities, where thermal wind relations are required to be exact, Hogg's model solves the velocities (streamfunctions) on all the vertical levels simultaneously. The dynamic equations are used just as normal constraints like the conservation constraints, and residuals are allowed in all the equations. Experiments 
have shown that the above formulation usually results in an over-determined equation system of full rank (Hogg, 1987; Zhang and Hogg, 1992; also experiments in the present work). The solutions are obtained in the least-squares sense by minimizing the residual norm of the equations. One does not face the problem of minimizing the solution (parameter) norm and the difficulty of justifying this minimization.

Because of the lack of the needed information in the real ocean with which to compare the inverse model results, the examination of the inverse model discussed above is done in the domain of the CME results. The hydrographic data in the numerical GCM oceans are used as input for the inverse model, and the estimated velocities and "diffusion" coefficients are compared to those values of the GCM oceans. Note that although the velocities and diffusion coefficients are known in the GCM oceans, they are not used to constrain the inverse solutions. Instead they are used to evaluate the functioning of the inverse model. In the real ocean, such information is generally not available.

The inverse model is first applied to a simpler, non-eddy resolving numerical GCM ocean, to get a basic understanding of the inverse model results and their reliability against the known parameter values. The grid resolutions, in both the numerical GCM and the inverse model, are $2^{\circ}$ longitude and latitude and 10 levels in the vertical, extending from the sea surface to the ocean bottom of $4000 \mathrm{~m}$. The numerical GCM domain is the North Atlantic Basin, and the subdomain in which the inverse model is applied is chosen as a quiet region in the subtropical eastern North Atlantic, extending from $42^{\circ} \mathrm{W}$ to $26^{\circ} \mathrm{W}$ and from $7^{\circ} \mathrm{N}$ to $25^{\circ} \mathrm{N}$. The large dissipation coefficients in the GCM $\left(1 \times 10^{7} \mathrm{~cm}^{2} / \mathrm{s}\right.$ for horizontal tracer diffusion and $1 \mathrm{~cm}^{2} / \mathrm{s}$ for vertical tracer diffusion) parameterize the sub-grid scale eddy processes. 
The GCM ocean state used to test the inverse model is the "equilibrium" state after 400 years of integration (Spall, 1992). The accuracy of the inverse model assumptions in this GCM ocean is first examined. It is shown that the oceanic circulations are in good agreement with the thermal wind balance except in the surface mixed layer ( 0 to $50 \mathrm{~m}$ depth). The area-averaged relative imbalances between the absolute velocity shear and the thermal wind shear are about $10 \%$ in the meridional direction and $20 \%$ in the zonal direction. The GCM fields do not exactly satisfy the steady inverse model conservation equations. After 400 years of integration (the atmospheric forcing is steady), the temporal variations are still important in the heat and salt balances, especially in the deep ocean where they are in the order of or even larger than the diffusion terms. The spatial structures of the temporal variation terms are similar to those of the vertical velocities in the deep ocean, and are very scattered in space and somehow simulate the random data noises.

In the first run of the experiments, the inverse model is applied to a six-layer water column immediately below the bottom of the surface mixed layer (the UpperLayer Model, or ULM), where the assumptions of the inverse model tend to have higher accuracy. The inverse model estimated horizontal circulations are consistent with the numerical GCM circulations within the estimated errors (which are very small) on all the vertical levels but the bottom one at $1250 \mathrm{~m}$ of this run, where the circulations are very weak (the area-averaged GCM velocity is $0.11 \mathrm{~cm} / \mathrm{s}$ ). These results are much better than Bigg's (1985) application of the beta-spiral method on a much simplified OGCM ocean (rectangular, flat-bottom ocean), where Bigg's inversions are significantly different from his GCM data (Fig.1.1) (the reasons will be summarized at the end). The estimated values for the horizontal and vertical 
diffusion coefficients, in the present work, are $A=(1.08 \pm 0.02) \times 10^{7} \mathrm{~cm}^{2} / \mathrm{s}$ and $K=1.03 \pm 0.01 \mathrm{~cm}^{2} / \mathrm{s}$. These estimates are very close to the data values (which are unity in the units shown above respectively), with relative errors of $8 \%$ and $3 \%$ accordingly. However, these small differences are significantly beyond the even smaller estimated errors.

The small but yet significant differences between the inversions and the GCM data are attributed to the different physics of the numerical GCM and of the inverse model. The thermal wind relation is accurate to about $15 \%$ in the GCM flow field. The GCM temporal variation terms in the heat and salt conservation equations, which are not included in the inverse model formulation, have larger magnitude than the diffusion terms on the deepest two levels of the above Upper-Layer model, although they are smaller than the diffusion terms on other (shallower) levels. In the overdetermined system of full rank, minimization of the equation residual norm forces the "missing" signals into the physical terms included in the inverse model, and thus biases the parameter solutions from their "true" values. The bias may not be significant for the parameters which appear in the terms which are larger than the "missing" signals, while significant for parameters appearing in the terms which are smaller than the "missing" signals. In a Lower-Layer model inversion (from 300 m to $2500 \mathrm{~m}$ ), by allowing $A$ to vary from depth to depth, it is shown that the solutions for $A$ are statistically consistent with the GCM value where the temporal variations are smaller than diffusion, whereas the differences between the solutions and the GCM value of $A$ are significantly out of the estimated error bounds where they are not. In a Full-Layer experiment with known temporal variations (as the right hand side of the equations), the inverse model estimates are totally consistent with the GCM data values for both circulations and diffusion coefficients. These 
experiments show how a correct inverse model could not fail to produce the correct answers, and how incorrect or biased answers could be resulted from an incorrect model. In the appearance of the non-white "noises", the inverse model solutions for the horizontal circulations are relatively robust, while those for the diffusive parameters are more sensitive to the "noises".

The Upper-Layer Model (ULM) estimated vertical velocities at the bottom of the surface mixed layer (the upper boundary of the ULM) are very similar to the GCM vertical velocities at the same depth, and both of them have the same spatial pattern and magnitude as the Ekman Pumping (vertical) Velocities (EPVs) $w_{e}$ (computed from the wind stress which was used in the GCM), but the values are different. It is shown that the small differences between the EPVs and the GCM vertical velocities (and also the ULM estimates) are in the order of the vertical velocities $\left(w_{g}\right)$ associated with the horizontal divergence of the geostrophic component of the horizontal absolute velocity in the surface mixed layer. $w_{g}$ is about $1 / 4$ of $w_{e}$. An experiment specifying the vertical velocity at the bottom of the mixed layer to be $\operatorname{EPV}\left(w_{e}\right)$ in the ULM shows that the solutions are highly biased from their "true" values. For example, the estimates for the diffusion coefficients, $A=(1.43 \pm 0.07) \times 10^{7} \mathrm{~cm}^{2} / \mathrm{s}$ and $K=(1.11 \pm 0.04) \mathrm{cm}^{2} / \mathrm{s}$, have very significant differences from the GCM values.

One of the inverse model assumptions, namely approximate thermal wind balance, does not hold in the surface mixed layer. Using Ekman theory, a surface layer model is developed by including the vertically integrated Ekman transport in the mass, heat, and salt conservation equations to estimate the heat and fresh water fluxes at the air-sea interface. This is done by incorporating the above surface layer model into the deeper layers, but the equations in the surface mixed layer are down 
weighted since they have larger uncertainties and more unknowns. The air-sea heat and fresh water fluxes estimated in this way are nicely consistent with those used in the GCM in both spatial structures and numerical values within the estimated errors. The geostrophic component $\left(\vec{u}_{g}\right)$ of the absolute velocity in the mixed layer is also kept as an unknown and estimated by the inversion. A circulation scheme composed of the estimated $\vec{u}_{g}$ and the vertically averaged wind driven circulation in the mixed layer is also in good agreement with the absolute horizontal circulation of the GCM ocean at $25 \mathrm{~m}$. The estimated solution errors are larger in the mixed layer than in the layers below, as there are more unknowns with the same number of constraints. The estimates for the parameters below the mixed layer are basically the same as those estimated from the Upper-Layer Model without the mixed layer (as the equations in the mixed layer are highly down-weighted), but the uncertainties are enlarged.

After examining its behaviors in various situations, the inverse model is applied to the whole water column (with 10 layers from sea surface to the ocean bottom of $4000 \mathrm{~m}$ ) in the horizontal domain concerned. It is shown that the estimated horizontal circulations are very similar to those in the GCM ocean in both spatial patterns and values from surface to bottom. On four levels at 300, 500, 800 , and $1250 \mathrm{~m}$, the estimated circulations are statistically consistent with the GCM ones. Differences on other levels, although very small, are significantly beyond the even smaller estimated errors. The estimates for diffusion coefficients, $A=(1.09 \pm 0.02) \times 10^{7} \mathrm{~cm}^{2} / \mathrm{s}$ and $K=1.03 \pm 0.02 \mathrm{~cm}^{2} / \mathrm{s}$, have similar characteristics. Given the accuracy of the inverse model physics in the GCM ocean (e.g., around $15 \%$ errors in the thermal wind relation, and the temporal variations in the heat and salt conservation equations are quite significant, especially at great 
depth), the inverse model results are very promising for the large scale circulations and diffusion coefficients.

The estimated vertical velocities from the Full-Layer Model do not have the small scale (actually very scattered) structures of the GCM vertical velocities. Instead they are very smoothed in space and are very similar to the 9-point spatially smoothed structures and values of the GCM vertical velocity. In fact, the differences are insignificant within the estimated error bars most of the time. The inverse model only picks up the large scale signals and filters out the "unknown" small scale "noises", unless the inverse model is explicitly forced by small scale signals.

By including the scattered signals of the temporal variation terms into the inverse model (specified as known right hand side), it is found that the small scale structures of the GCM vertical velocity are recovered by the inversion. This is due to the fact that in the GCM ocean, the large amplitude "peaks" in the vertical advection terms are only balanced by the large amplitude "peaks" in the temporal variation terms. In this case the inverse model estimates are all consistent with the GCM parameter values within the estimated errors, despite the existence errors in the thermal wind relation. For example, the estimated the diffusion coefficients are $A=(1.03 \pm 0.05) \times 10^{7} \mathrm{~cm}^{2} / \mathrm{s}$ and $K=0.99 \pm 0.02 \mathrm{~cm}^{2} / \mathrm{s}$.

Experiments on the parameterization of the variables in the inverse model are carried out in the presence of the data "noise" (the temporal variation terms). It is shown that the solutions for the horizontal and vertical velocities $\overrightarrow{\mathbf{u}}, w$ are relatively robust, while those for the diffusive parameters $A, K$ (especially for $A$ ) are sensitive to the specific form of the parameterization (also see, for example, Olbers, 1989). Parameterizing $A, K$ and air-sea fluxes as third order polynomials while keeping 
other variables (the velocities) as point-wise unknowns results in plausible solutions for all variables on all levels except the $A$ on the deepest three levels, where the "unknown noises" are larger than diffusion terms and non-white.

The application of the inverse model in the above non-eddy resolving numerical GCM ocean showed that the inverse model is quite capable of recovering the GCM circulations and turbulent diffusion coefficients from the hydrographic data. However, the real oceans are under seasonally varying forcing and full of eddy activity. The ubiquity of meso-scale motions and their importance in the ocean general circulation are well established facts in oceanography. The sparsity of current measurements precludes direct estimates of the eddy contribution in heat, salt, and other tracer budgets. The role that ocean eddies play in global climate is still an open question, and this is addressed in the state-of-the art Community Modeling Effort (CME). Examination of inverse model behaviors in such an eddy-resolving numerical GCM (EGCM) ocean is more meaningful and relevant to the application of the inverse models in the real ocean.

The EGCM ocean was generated by running the GFDL primitive equation model of the ocean with a grid resolution of $2 / 5^{\circ}$ longitude by $1 / 3^{\circ}$ latitude and by 30 levels in the vertical with "real" topography (Bryan, personal communication). The horizontal GCM domain is the North Atlantic Basin from $15^{\circ} \mathrm{S}$ to $65^{\circ} \mathrm{N}$. The horizontal dissipation is highly scale-selective biharmonic, with coefficients of $-1.0 \times$ $10^{19} \mathrm{~cm}^{4} / \mathrm{s}$ for momentum and of $-2.5 \times 10^{19} \mathrm{~cm}^{4} / \mathrm{s}$ for tracers. The vertical dissipation is the Laplacian operator with coefficients of $10 \mathrm{~cm}^{2} / \mathrm{s}$ and $0.3 \mathrm{~cm}^{2} / \mathrm{s}$ for momentum and tracers respectively. The model was integrated for 24 years, with initial conditions which were the solution from a previous run, with higher viscosity, at an intermediate time. The evolving model solution was sampled at a 3 -day time 
interval during the final five years of the simulation. The 5-year, 600-sample means were taken as the "climatological" means in this study.

The inverse model is first applied to the time-mean fields of the EGCM ocean, using the same fine grid-resolution of the EGCM and with the objective to examine whether the inverse model solutions from the time-mean hydrographic data represent the time-mean parameter values of the EGCM ocean. The inverse model domain is chosen from $34.8^{\circ} \mathrm{W}$ to $31.6^{\circ} \mathrm{W}, 16.17^{\circ} \mathrm{N}$ to $19.17^{\circ} \mathrm{N}$, and from $92 \mathrm{~m}$ to $2125 \mathrm{~m}$ in the vertical (13 vertical levels). The number of grid points on which the conservation equations are formulated is $8 \times 9 \times 13$. The small horizontal domain is limited by the CPU memory capacity of the computer resources (the CRAYs at MIT and NCAR). This large memory is required for the SVD analysis of the equation coefficient matrix (the data), in order to decide how the solutions should be obtained. Although the solutions themselves can be obtained by other methods (the matrix is sparse), they are insufficient for a complete analysis of the inverse model (especially for the "testing" purpose). In this work we sacrifice the horizontal domain to include more vertical levels, as experiments showed that the inverse model results are more sensitive to the number of vertical levels and relatively insensitive to the size of the horizontal domain.

The accuracy of the inverse model equations in the time-mean fields of the EGCM ocean is first examined. It is shown that the thermal wind relation is well satisfied, with relative imbalances of about $5 \%$. Although the time mean is taken over a five-year period, it is shown that temporal variations, compared to the vertical eddy fluxes, are still important terms in the heat and salt conservation equations, especially in the deep layers. The biharmonic horizontal dissipation, which is also not explicitly included in the inverse model, is significant compared to the eddy 
fluxes too in the fine grid resolution. As the orders of taking the time mean and spatial gradient/divergence are commutable, the continuity equation is, as in the instantaneous fields, exactly satisfied by the time-mean velocity fields.

The inverse model estimated horizontal circulations have similar flow patterns and magnitudes as those of the time-mean EGCM circulations. Although there are some differences, the values are completely consistent with each other within their statistical error ellipses on all the 13 levels. It is interesting to notice that, although both the inversion of the time-mean hydrographic data and the direct time mean (average) of the instantaneous EGCM horizontal velocity result in similar flow patterns (with similar magnitudes), the uncertainties (errors) are very different. On one hand, the statistical errors of the direct time-mean circulations are very large, and the uncertainty of the flows are high. In fact, at the deep levels, the horizontal velocity vectors are totally within the error ellipses (with $95 \%$ confidence. see Fig. 4.5), and statistically we really do not know where the flows go (or they can go in any directions). On the other hand, the estimated errors (uncertainties) from the inverse model are very small, and the flow patterns are statistically well defined on all the vertical levels.

The large uncertainties (errors) in the direct time-mean circulations are caused by the very energetic (high frequency) eddy flow fields, and the small uncertainties (errors) in the inverse estimates (from the time-mean hydrographic data) are associated with the small uncertainties in the time-mean tracer (temperature and salinity) fields (section 4.2). This is consistent with the concept that tracers have very low frequency variations. In this sense, we can say that the inverse model succeeded in extracting (resolving) the "ocean general circulation" from the "climatological" hydrographic data. By resolving it is meant here that the parameters are 
determined with statistical significance (solutions are significantly above the errors or uncertainties).

The vertical velocities estimated by the inverse model are of the same order and in the same spatial distribution patterns as those of the EGCM time means in the upper layers. At great depths, the estimates are visually different from the EGCM data, but the differences are insignificant within the statistical errors for the time means and the estimated errors for the inverse estimates.

In the inverse model, the assumption of Fickian diffusion with isotropic horizontal diffusion coefficients is used with the intention to parameterize the eddy fluxes. In the inversion the diffusion coefficients are parameterized as polynomial functions in space. Although the inverse model estimated "diffusion" coefficients are in the same order as those of the eddy diffusion coefficients directly computed from the GCM eddy fluxes at shallow depths, they are very different at great depths most of the time, although the differences are only marginally significant. The significant discrepancies are accounted by the fact that the inverse model estimated "diffusion" coefficients parameterize not only the effects of the eddy fluxes in the conservation equations, but also those not explicitly included in the inverse model (e.g., the temporal variation and the biharmonic dissipations). In this sense the inverse model estimated "diffusion" coefficients are not the eddy diffusion coefficients any more.

In the real ocean, spatial smoothing (or objective mapping) is used in almost all the climatological data sets. Given the essentially red spectrum of the ocean, it makes sense to look for smooth solutions, such as for the large scale ocean general circulation. Smoothed constraints also act to extend information into regions where 
solution may be indeterminate (e.g., Thacker, 1988). However, when the inverse model is applied to the spatially smoothed hydrographic data with larger grid spacing, the interpretation of the inverse model results becomes more complicated, as, theoretically, the orders of doing inverse and of taking spatial smoothing are generally not commutable. The implied hypotheses in applying inverse models in the real ocean are that the estimated circulations from the spatially smoothed time-mean (climatological) hydrographic (and other tracer) data could be interpreted as the spatially smoothed time-mean (climatological) oceanic circulations, and the estimated "diffusion" coefficients could be interpreted as the eddy diffusion coefficients. The validity of these hypotheses is examined in the last part of this thesis.

A horizontal Gaussian smoothing with radius of six points $\left(2.4^{\circ}\right.$ longitude $2^{\circ}$ latitude) is applied to the 5 -year time means of the fine grid resolution $\left(2 / 5^{\circ}\right.$ longitude by $1 / 3^{\circ}$ latitude) EGCM ocean to generate a spatially smoothed timemean data set. The inversion is done in a domain extending from $42.2^{\circ} \mathrm{W}$ to $23.0^{\circ} \mathrm{W}$, $8.67^{\circ} \mathrm{N}$ to $26.67^{\circ} \mathrm{N}$, and from $92 \mathrm{~m}$ to $2125 \mathrm{~m}$ in the vertical, with grid resolution of $2.4^{\circ}$ longitude by $2.0^{\circ}$ latitude and by 13 layers in the vertical.

The time-mean velocities satisfy the continuity equations exactly in the fine grid resolution of the EGCM. However, this is not true for the spatially smoothed time-mean velocities in the coarse grid resolution, mainly due to the subsampling aliasing effects (as shown in Appendix A, the aliasing of large grid spacing is greatly reduced by spatial smoothing). The imbalances (from the 3-D divergence) are small in the upper layers, but quite large in the deep layers. The imbalances between the shears of the spatially smoothed absolute velocity and the thermal wind shears from the spatially smoothed density field are also increased (from $5 \%$ of the fine grid resolution time means to $9 \%$ of the coarse grid resolution spatially smoothed 
time means), but the smoothed flows are still approximately in the thermal wind balance. With the larger grid spacing, the biharmonic dissipations are greatly reduced, but again the temporal variation terms are still significant compared to the vertical eddy flux terms.

With the spatial smoothing, the statistical errors for the smoothed horizontal circulations are greatly reduced. Even with these much smaller error ellipses, the inverse model estimated horizontal circulations from the smoothed time-mean hydrographic data are generally consistent with the smoothed time-mean circulations of the EGCM. Total consistency is achieved on the eight upper levels above $722 \mathrm{~m}$, and below, consistency is achieve in most of the area - significant differences only occur at few grid points. The large scale flow patterns of the estimations and of the smoothed EGCM ocean are the same on all the 13 vertical levels.

The estimates of the vertical velocities are much improved towards the smoothed time-mean vertical velocities (than in the case of the inversion of the unsmoothed time means in the fine grid resolution). The large scale structures are very similar, especially on the upper levels. The values are also completely consistent with each other in the upper layers, even the statistical errors of the smoothed time-mean vertical velocities are greatly reduced. In the deep layers, significant differences occur at the northwest corners, and the estimates are consistent with the smoothed time-mean vertical velocities in other areas.

In summary, we conclude this chapter with the following points and comments:

1. Inverse model solutions for the horizontal circulation are relatively robust. In all the cases discussed in this work, inverse estimates for the horizontal 
circulations are statistically consistent with the "data" most of the time. Horizontal advections are almost always dominant terms in the tracer budgets, thus it is easier to extract the values of the horizontal velocities from these signals. Also, in addition to the conservation law constraints, horizontal circulations are also constrained by the dynamic equations (thermal wind balances).

2. On the other hand, the determination of the eddy diffusion coefficients is more difficult. The difficulty lies in the fact that, at least for the cases studied in this work, the signals from the eddy flux terms are mixed with or even disguised by the signals from the terms neglected in the steady inverse model (e.g., the temporal variation terms). Minimization of the equation residual norm in obtaining the inverse model solution forces the solutions for the "diffusion" coefficients of the inverse model to be biased from the values of the real eddy diffusion coefficients. In order to get better solutions for the eddy diffusion coefficients, we need either to search for ways to parameterize the temporal variation (and other neglected) terms in the steady inverse model, if they show significance in the tracer budgets, or to use time-dependent inverse models.

3. Experiments showed that, although the inversion of the time-mean hydrographic data and the direct time mean (average) of the velocity time series both results in the same time-mean horizontal circulation schemes, the confidence we can put on them are very different. On one hand, the uncertainties for the directly time-averaged circulations are very large, and in the case studied in this work, statistically the time-mean circulations can flow in any directions most of the time at great depth. On the other hand, the uncertainties for the inverse model estimated horizontal circulations are very small, and the flow regimes are well defined in the presence of the estimated errors. Therefore the inverse model estimated circulation 
from the time-mean hydrographic data might be representative of the "climatological" state of the ocean. This is related to the fact that hydrographic and tracer data have very slow variations, while motions are more energetic.

4. The test of the hypothesis that the circulation deduced from a climatological (usually spatially smoothed time mean) hydrographic data set could be interpreted as the large scale oceanic general circulation is achieved. The estimated horizontal circulations from the inverse model from the spatially smoothed timemean hydrographic data are quite consistent with the directly spatially-smoothed time-mean circulations.

5. Experiments showed that the inverse model results are sensitive to the number of the vertical layers (levels) and relatively insensitive to the size of the horizontal domains.

Over all, in this study both the applications of the inverse model in the noneddy resolving GCM ocean and the eddy-resolving GCM ocean revealed that the inverse model is quite capable of producing the correct (spatially smoothed large scale) ocean general circulations from the (spatially smoothed time-mean) hydrographic data, and also the correct diffusion coefficients in the case that the data "noises" in the equations are smaller that the advection and diffusion terms. These results are very different from the conclusions from Bigg (1985) and Killworth and $\operatorname{Bigg}(1988)(\mathrm{KB})$. There are various possible reasons for the discrepancy between our conclusion and those of Bigg and KB.

First of all, the inverse model used in this work is formulated in a more accurate way than the original beta-spiral method. In Bigg and KB's beta-spiral inverse models, the (horizontal) eddy (diffusion) fluxes (which were included in the 
present work) were ignored, and thus the inverse models are very likely insufficient to represent the GCM oceans, as shown in the present work. It was also shown in the present work that the temporal variations are important players in the heat and salt balances in both the non-eddy resolving GCM ocean and the 5-year time means of the EGCM ocean. It is unclear how important these terms are in Bigg and KB's GCM oceans. Further, point-wise density conservation equations were used in Bigg, and thus large scale water properties, such as conservation of mass flow between hydrographic stations, were not necessarily guaranteed. In the present work, mass (as well as heat and salt) were conserved in all the individual finite difference boxes and thus also large scale sections. In addition to the mass conservation used in Bigg and $\mathrm{KB}$, heat and salt conservation equations were also added in the present work. These constraints provided more information in determining the inverse model solutions for the ocean circulations, and especially for the diffusive parameters. Exact thermal wind balances were required in Bigg and $\mathrm{KB}$, while residuals were allowed in the thermal wind balances in the present work.

Secondly, it was shown in the present work that aliasing of subsampling/large grid spacing could lead to large noises in the finite difference equations with coarse grid resolutions. It is shown in Appendix A that these noises can be greatly reduced by spatial smoothing. In Bigg and KB's inversions with coarse grid resolutions, they did not mention anything about spatial smoothing. Unsmoothed data could lead to further biases of the parameter solutions. Moreover, experiments showed that the inverse model results were sensitive to the exact finite difference forms used in the model, especially in the vertical direction. For example, due to the non-constant spacing and the interplay of the $w$ and $T(S, u, v)$ surfaces in the vertical in the GCM, interpolating $T$ on the $w$ surfaces was necessary in order to compute the vertical 
advection fluxes $w T$. The results of a linear interpolation in depth (or distanceweighted averaging) were different from those of a simple arithmetic averaging as used in the GCM. In the present work, all the finite difference schemes were kept as the same as those in the GCM. It is unclear how this issue was dealt with in Bigg and KB. In the real ocean, properly representing the tracer fields, especially the vertical profiles, is essentially important to the inverse model solutions.

Thirdly, there are some issues related to the specific inverse techniques. For the over-determined systems (e.g., the beta-spiral models), it is unclear how the equations were weighted/scaled in Bigg and KB. For the under-determined systems (e.g., the box inverse model), KB claimed that the equation system was of full rank, and thus all the equations of the inverse model were satisfied exactly by the inverse solutions. But in fact, the inverse model did not perfectly represent the GCM ocean (without errors).

Finally, it should be pointed out that the issue of whether a GCM ocean is properly represented by an inverse model should be distinguished from the issue of whether the real ocean is properly represented by the same inverse model. This is because that the numerical GCM oceans do not perfectly represent the real ocean. In "testing" an inverse model (which is usually formulated for the real ocean) in a GCM ocean, one should first examine how accurate the assumptions of the inverse model are in the GCM ocean. If the inverse model physics is statistically different from that of the GCM ocean, one would not expect to get the correct or unbiased answers from the statistical inferences for all the parameters. As the GCM ocean is not the same as the real ocean, failure of representing a GCM ocean by an inverse model does not guarantee failure of representing the real ocean by the same inverse model, and vice versa. In both the non-eddy resolving and eddy-resolving 
GCM oceans, the importance of the temporal variation terms in the heat and salt conservation equations indicates that the the GCM oceans were still in the process of spinning up. In the real ocean, a climatological data set is usually produced using data collected over a limited time period, and the temporal variations related to the limited period of time means could be important error sources for steady inverse models. 


\section{APPENDIX A: SPATIAL SMOOTHING AND FINITE-DIFFERENCE GRADIENT}

1. Inconsistent schemes used in the spatial smoothing and spatial gradient may cause differences in the gradients of the smoothed parameters and the smoothing of the gradients of the unsmoothed parameters.

$\begin{array}{ccccc}x & x & x & x & x \\ x & & x, j+1 & & \\ & i-1, j & i, j \quad i+1, j & \\ x & x & \text { x } & x & x \\ x & x & x & x & x\end{array}$

Fig. A.1. 5-point average

This point can be illustrated by a simple example as shown in Fig. A.1. For simplicity, define the spatial smoothing as a 5-point arithmetic mean:

$$
\bar{T}_{i, j}=\frac{1}{5}\left(T_{i, j}+T_{i-1, j}+T_{i+1, j}+T_{i, j-1}+T_{i, j+1}\right) .
$$

Then the centered-finite difference form of the zonal gradient of the smoothed field is

$$
\begin{aligned}
& \frac{\partial \bar{T}}{\partial x}=\frac{\bar{T}_{i+1, j}-\bar{T}_{i-1, j}}{2 \Delta x_{j}} \\
= & \frac{\frac{1}{5}\left(T_{i+1, j}+T_{i, j}+T_{i+2, j}+T_{i+1, j-1}+T_{i+1, j+1}\right)-\frac{1}{5}\left(T_{i-1, j}+T_{i-2, j}+T_{i, j}+T_{i-1, j-1}+T_{i-1, j+1}\right)}{2 \Delta x_{j}}
\end{aligned}
$$




$$
=\frac{1}{5}\left[\frac{T_{i+1, j}-T_{i-1, j}}{2 \Delta x_{j}}+\frac{T_{i, j}-T_{i-2, j}}{2 \Delta x_{j}}+\frac{T_{i+2, j}-T_{i, j}}{2 \Delta x_{j}}+\frac{T_{i+1, j-1}-T_{i-1, j-1}}{2 \Delta x_{j}}+\frac{T_{i+1, j+1}-T_{i-1, j+1}}{2 \Delta x_{j}}\right],
$$

where $\Delta x_{j}$ is the step-size in the zonal direction as function of latitude (for step-size of constant in degrees, the zonal step-size in distance varies as a cosine function of latitude). On the other hand, the direct spatial mean of the gradients is

$$
\begin{aligned}
& \left(\frac{\overline{\partial T}}{\partial x}\right)_{i, j}=\frac{1}{5}\left[\left(\frac{\partial T}{\partial x}\right)_{i, j}+\left(\frac{\partial T}{\partial x}\right)_{i-1, j}+\left(\frac{\partial T}{\partial x}\right)_{i+1, j}+\left(\frac{\partial T}{\partial x}\right)_{i, j-1}+\left(\frac{\partial T}{\partial x}\right)_{i, j+1}\right] \\
= & \frac{1}{5}\left[\frac{T_{i+1, j}-T_{i-1, j}}{2 \Delta x_{j}}+\frac{T_{i, j}-T_{i-2, j}}{2 \Delta x_{j}}+\frac{T_{i+2, j}-T_{i, j}}{2 \Delta x_{j}}+\frac{T_{i+1, j-1}-T_{i-1, j-1}}{2 \Delta x_{j-1}}+\frac{T_{i+1, j+1}-T_{i-1, j+1}}{2 \Delta x_{j+1}}\right] .
\end{aligned}
$$

It can be seen that the difference between $\frac{\partial \bar{T}}{\partial x}$ and $\left(\frac{\overline{\partial T}}{\partial x}\right)$ lies in the variation of the zonal step-size with latitude in the last two terms. 


\section{Aliasing associated with the under-sampling in the coarser- grid resolution scheme.}

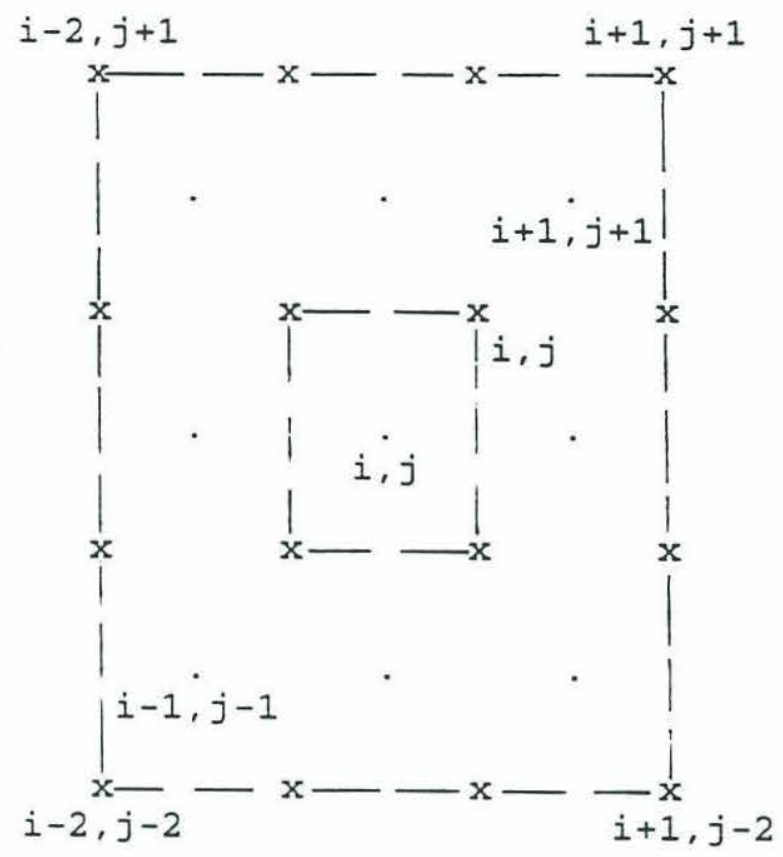

Fig. A.2. u,v at $x$ points; $w$ at . points

As shown in Fig. A.2, suppose the velocity fields are 3-dimensionally nondivergent in the fine-grid resolution (in the small box) (In the numerical GCM, the vertical velocities are actually diagnostically computed from the horizontal velocity divergence on the fine-grid resolution). In finite difference form, assume the velocity divergence is balanced as

$$
\begin{gathered}
\frac{\frac{1}{2}\left[\left(u_{i, j}+u_{i, j-1}\right)-\left(u_{i-1, j}+u_{i-1, j-1}\right)\right]}{\Delta x_{j}}+\frac{\frac{1}{2}\left[\left(v_{i, j}+v_{i-1, j}\right)-\left(v_{i, j-1}+v_{i-1, j-1}\right)\right]}{\Delta y} \\
+\frac{w_{i, j, k+1}-w_{i, j, k}}{\Delta z}=0 .
\end{gathered}
$$

However, in a coarser-grid resolution, say the grid spacing is extended one step-size on each side of the box (Fig. A.2), the same difference scheme results in horizontal 
velocity divergence as

$$
\frac{\frac{1}{2}\left[\left(u_{i+1, j+1}+u_{i+1, j-2}\right)-\left(u_{i-2, j+1}+u_{i-2, j-2}\right)\right]}{3 \Delta x_{j}}+\frac{\frac{1}{2}\left[\left(v_{i+1, j+1}+v_{i-2, j+1}\right)-\left(v_{i+1, j-2}+v_{i-2, j-2}\right)\right]}{3 \Delta y},
$$

while the vertical velocity divergence is still calculated as above, and thus these two velocity divergences generally do not balance each other exactly-residual or imbalance exists. Various spatial smoothing or averaging can reduce the size of the residual, but there is no guarantee that the residual will vanish. This will be further illustrated in the following simple example.

\section{A simple example}

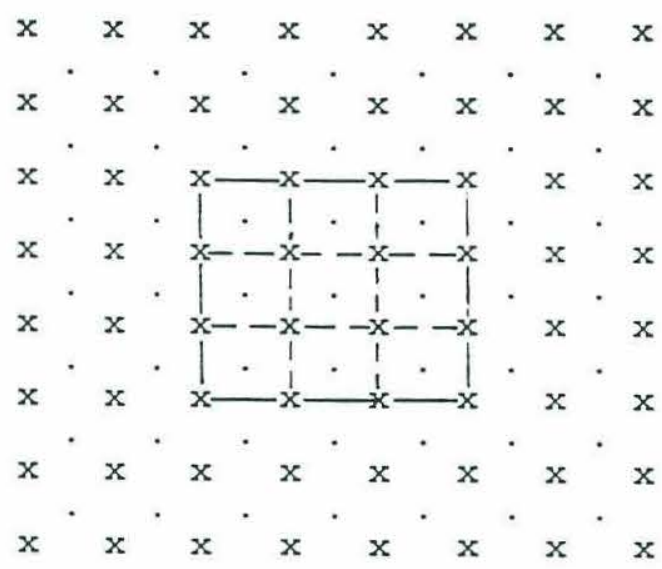

A simple example is designed to illustrate the two points made above. A 3-D velocity field is generated by a random data generator, requiring it to be 3dimensionally nondivergent on a fine grid resolution of $37 \mathrm{~km} \times 37 \mathrm{~km} \times 200 \mathrm{~m}$ (which is about $1 / 3$ degrees in the horizontal) (Fig. A.3). The horizontal velocities $u, v$ 
are located at the " $\mathrm{x}$ " points (the total point number is $8 \times 8$ ), and the vertical velocities at the top and bottom (only one layer) are located at the centers (the . points, with a total number of $7 \times 7)$.

Constructed in this way, the 3 -D velocity divergences on the (constant) finegrid resolution vanish, as shown in Exp.1 of Table A.1.

In the next experiment (Exp.2), effects of variation of zonal step-size with latitude is examined, to illustrate the first of the points made above. Note that in Exp.1 (3-D nondivergent), the step-sizes are all constant. The 3-D velocity divergences with varying zonal step-size, assuming the area ranges from $15^{\circ} \mathrm{N}$ to $17^{\circ} \mathrm{N}$ with a step-size of $1 / 3^{\circ}$, are shown in Exp.2 of Table A.1. With this inconsistent difference scheme (with Exp.1), residuals/imbalances exist in the continuity equation. But it will be shown that these imbalances are much smaller than those caused by the aliasing in the larger-grid spacing (Exp.3).

The third experiment (Exp.3) uses the exact difference scheme as in Exp.1 but with larger-grid spacing (Fig. A.2), aiming at illustrating the second of the points made above. As shown in Exp.3 of Table A.1, with the coarser-grid resolution, residuals/imbalances exist in the continuity equation, and these residuals are larger than those caused by the variation effects of the zonal step-size.

In the fourth experiment (Exp.4), ways are sought to reduce the aliasing effect due to the subsampling as in Exp.3. In this experiment, the vertical velocity divergence at the center of the box (Fig. A.2) is taken as the 9-point arithmetic mean:

$$
\begin{aligned}
\overline{w_{z}}(i, j)= & \frac{1}{9}\left[w_{z}(i, j)+w_{z}(i-1, j)+w_{z}(i+1, j)+w_{z}(i-1, j-1)+w_{z}(i, j-1)\right. \\
& \left.+w_{z}(i+1, j-1)+w_{z}(i-1, j+1)+w_{z}(i, j+1)+w_{z}(i+1, j+1)\right],
\end{aligned}
$$


while horizontal velocity divergences are computed from the mean velocities on the four boundaries. For example, at the west boundary, the zonal velocity is taken as $\left(u_{i-2, j+1}+u_{i-2, j}+u_{i-2, j-1}+u_{i-2, j-2}\right) / 4$. The horizontal resolution is the same as in Exp.3 (i.e. the larger-grid spacing). It can be seen from Exp.4 of Table A.1 that the residuals in the continuity equation are reduced, but they still do not vanish.

The last two experiments are carried out on the spatially smoothed velocity field of $u, v, w$. The smoothed field is generated by a non-uniform weight function of the form

$$
\bar{u}_{i, j}=\frac{1}{2} u_{i, j}+\frac{1}{8}\left(u_{i-1, j}+u_{i+1, j}+u_{i, j-1} u_{i, j+1}\right) .
$$

In Exp.5 of Table A.1, the difference scheme is exactly the same as in Exp.1 (e.g., the step-sizes are all constant and on the fine-grid resolution), but now it is for the smoothed velocity field. When the spatial smoothing and spatial gradient schemes are consistent, the orders of taking spatial smoothing and spatial gradient commute, and thus the residuals in the continuity equation also vanish for the smoothed velocity field on the fine-grid resolution (Exp.5 of Table A.1).

Aliasing due to under-sampling in the coarser-grid resolution still exists for the spatially smoothed velocity field. In Exp.6 the scheme is the same as in Exp.5 but with coarser-grid resolution (as in Exp.3). It can be seen from Exp.6 of Table A.1 that residuals in the continuity equation in the larger grid spacing do not vanish. This experiment is an analog of Exp.3 for the unsmoothed velocity field, but it can be seen that the imbalances in the smoothed velocity divergences (Exp.6 of Table A.1) are much smaller than the imbalances in the unsmoothed velocity 
divergences. It is also noticed that in this data set, the imbalances in the divergences of the smoothed velocity (with a non-uniform weight function) (Exp.6 of Table A.1) are smaller than the imbalances in Exp.3 of Table A.1-those computed from the arithmetic means of the unsmoothed data.

TABLE A.1: Experiments on Velocity Divergence

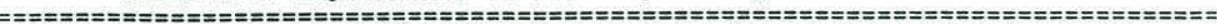

Exp.1: Velocity divergence in the fine-grid resolution

\begin{tabular}{rrrrrrr}
$1.0 \mathrm{e}-21$ & $*$ \\
0.0126 & 0.0136 & 0.0004 & 0.0029 & 0.0386 & -0.0013 & -0.0323 \\
0.0120 & 0.1042 & -0.0104 & 0.0214 & 0.0011 & 0.0851 & -0.0220 \\
-0.0116 & -0.0334 & 0.0422 & -0.0014 & -0.0221 & 0.0064 & 0.0061 \\
-0.0824 & -0.0047 & 0.0999 & -0.0963 & -0.0006 & -0.0403 & -0.0126 \\
-0.0716 & 0.0134 & 0.0131 & -0.0903 & 0.0111 & -0.0189 & 0.0430 \\
-0.0108 & 0.0027 & 0.0065 & 0.0450 & -0.0264 & -0.0070 & -0.0296 \\
0.0758 & -0.0469 & 0.0053 & -0.0350 & -0.0075 & -0.0069 & -0.0052 \\
\hline \\
Exp.
\end{tabular}

Exp.3: Same as Exp.1 but with coarser-grid resolution ---effects (aliasing) of the under-sampling

$\begin{array}{rrrrr}1.0 \mathrm{e}-05 & & & & \\ 0.1580 & -0.0934 & -0.0530 & 0.0728 & -0.0941 \\ 0.2288 & -0.0968 & 0.0731 & 0.0501 & -0.1363 \\ 0.2524 & -0.1983 & 0.1886 & -0.1398 & 0.0472 \\ 0.1579 & -0.1707 & 0.1503 & -0.2052 & 0.1757 \\ 0.0855 & -0.0588 & 0.1152 & -0.1197 & 0.1530\end{array}$

Exp.4: Same as Exp.3 but the divergences are computed as the arithmetic means---averaging of the under-sampling aliases

$\begin{array}{rrrrr}1.0 \mathrm{e}-06 & & & & \\ 0.3949 & -0.2335 & -0.1326 & 0.1821 & -0.2351 \\ 0.5720 & -0.2419 & 0.1826 & 0.1253 & -0.3408 \\ 0.6310 & -0.4958 & 0.4714 & -0.3496 & 0.1180 \\ 0.3947 & -0.4267 & 0.3759 & -0.5130 & 0.4391 \\ 0.2136 & -0.1469 & 0.2881 & -0.2993 & 0.3825\end{array}$

Exp.5: Same as Exp.1 but for the spatially smoothed velocities

$\begin{array}{rrrrr}1.0 \mathrm{e}-21 & * & & & \\ 0.3044 & -0.0969 & -0.2070 & -0.0625 & 0.0137 \\ -0.2308 & -0.0506 & -0.1637 & -0.0597 & -0.1690 \\ -0.2712 & 0.0652 & 0.0160 & 0.1999 & -0.0539 \\ 0.3374 & 0.6621 & -0.2162 & -0.0667 & 0.2846 \\ 0.0883 & -0.1224 & -0.3579 & -0.1333 & 0.1586\end{array}$

Exp. 6: Same as Exp. 5 but with coarser-grid resolution

$\begin{array}{rrr}1.0 \mathrm{e}-06 & * & \\ 0.1120 & 0.0696 & 0.0179 \\ 0.1025 & 0.0640 & -0.0845 \\ 0.0383 & -0.0371 & -0.1149\end{array}$




\section{Acknowledgments}

First of all I would like to thank my thesis advisor, Nelson Hogg, for his patient guidance and support, many invaluable discussions, and the freedom he has given me along the way. His enthusiasm and generous encouragement have been a constant source of inspiration, and his deep insight and imagination have been a great help for me. I am grateful to his encouragement in having the "privilege" of always being the first one reading and correcting the drafts I wrote.

I would also like to thank the members of my thesis committee - Jochem Marotzke, Brechner Owens, Michael Spall, and Carl Wunsch, and the defense chairman, Rui Xin Huang, for many valuable suggestions concerning various aspects of the thesis, and oceanography in general. Discussions with Trevor McDougall are also very helpful. Their constructive criticism of earlier drafts of this thesis have greatly improved the accuracy and the readability of the text.

I am grateful to Mike Spall for providing me the non-eddy resolving GCM ocean data, Frank Bryan the eddy-resolving GCM ocean data, and their and Xin Huang's help in getting familiar with the GFDL model. Dave Chapman's kind help makes me getting around the NCAR CRAY computer much easier.

Along the way in the Joint Program, I have got lot of help from the WHOI and MIT faculty. Steve Lentz served as my first summer advisor, and Mike McCartney and Harry Bryden served in my thesis committee in the early years. I cannot forget all kinds of help from Abbie Alvin in the Education Office. Friendship from the Joint Program students is invaluable. I would like to give my special thanks to Kwoklin Lee for her help in the preparation of the manuscript, and Joe LaCasce and Young-Gyu Park for their help at various occasions. I would also like to thank 
Barbara Gaffron and Anne-Marie Michael for their invaluable assistance when I need it.

Last but not least I would like to thank my family and friends for their support throughout my stay in the Joint Program. Their continual encouragement from the other side of the earth have kept me on going during difficult times.

This research was carried out under National Science Foundation grant OCE90-04396. Computations are partially realized on the NCAR CRAY and MIT CRAY super computer facility. 


\section{References}

Arakawa, A., and V.R. Lamb, 1977. Computational design of the basic dynamical processes of the UCLA general circulation model. Methods in Comput. Phys., 17, Academic Press, 174-265.

Backus, G.E. and J.F. Gilbert, 1967. Numerical application of a formalism for geophysical inverse theory. Geophys. J. Roy. Astron. Soc., 13, 247-276.

Bendat, J.S., and A.G. Piersol, 1986. Random Data: Analysis and Measurement Procedures. 2nd ed. Wiley-Interscience, New York, 407pp.

Bennett, A.F., 1992. Inverse Methods in Physical Oceanography, Cambridge University Press, Cambridge/New York. xvi, 349pp.

Bigg, G.R., 1985. The beta-spiral method. Deep-Sea Research, 32, 465-484.

Bretherton, F.P., R.E. Davis, and C. Fandry, 1976. A technique for objective analysis and design of oceanographic instruments applied to MODE-73. DeepSea Research, 23, 559-582.

Bryan, F., 1987. Parameter sensitivity of primitive equation ocean general circulation models. Journal of Physical Oceanography, 17, 970-985.

Bryan, F., and W. Holland, 1989. A high-resolution simulation of the wind- and thermohaline-driven circulation in the North Atlantic Ocean. In: Parameterization of Small-scale Processes, Muller, P. and D. Henderson (eds), 'Aha Huliko'a Proceedings, Univ. of Hawaii at Monoa, 99-116.

Bryan, K., 1969. A numerical method for the study of the circulation of the world ocean. J. Comput. Phys., 4, 347-376.

Bryan, K., 1979. Models of the world ocean circulation. Dynamics of Atmosphere and Oceans, 3, 327-338.

Bryan, K., and M.D. Cox, 1972. An approximate equation of state for numerical models of ocean circulation. Journal of Physical Oceanography, 2, 510-514.

Bryden, H.L., 1977. Geostrophic comparisons from moored measurements of current and temperature during the Mid-Ocean Dynamics Experiment. Deep-Sea Research, 24, 667-681.

Bryden, H.L., J. Candela and T.H. Kinder, 1994. Exchange through the Strait of Gibraltar. Prog. Oceanogr., 33, 201-248. 
Cox, M.D., 1984. A primitive equation, 3-dimensional model of the ocean. GFDL Ocean Group Tech. Rep. No. 1, 143pp.

Cox, M.D., 1985. An eddy resolving numerical model of the ventilated thermocline. Journal of Physical Oceanography, 15, 1312-1324.

de Boor, C. 1978. A Practical Guide to Splines, Springer-Verlag, New York.

Defant A. 1941. Quantitative Untersuchungen zur Statik und Dynamik des Atlantischen Ozeans. Die absolute Topographie des physikalischen Meeresniveaus und der Drückflachen sowie die Wasserbewegungen im Raum des Atlantischen Ozeans. In Wissenschaftliche Ergebnisse der Deutschen Atlantischen Expedition auf dem Forschungs-und Vermessungsschiff "Meteor" 1925-1927, 6: 2nd Part, 1, pp.191-260.

Fiadeiro, M.E., and G. Veronis, 1984. Obtaining velocities from tracer distributions. Journal of Physical Oceanography, 14, 1734-1746.

Figueroa, H.A., and D.B. Olson, 1989. Lagrangian statistics in the South Atlantic as derived from SOS and FGGE drifters. Journal of Marine Research, 47, $525-546$.

Fu, L.L., 1981. The general circulation and meridional heat transport of the subtropical South Atlantic determined by inverse methods. Journal of Physical Oceanography, 11, 1171-1193.

Gaspar, P., J.C. Andre, and J.M. Lefevre, 1990. The determination of latent and sensible heat fluxes at the sea surface viewed as an inverse problem. Journal of Geophysical Research, 95, No. C9, 16169-16178.

Green, J.S.A., 1970. Transfer properties of the large-scale eddies and the general circulation of the atmosphere. Quart. J. Royal Meteor. Soc., 96, 157-185.

Haidvogel, D.B., and P.B. Rhines, 1983. Waves and circulation driven by oscillatory winds in an idealized ocean basin. Geophys. and Astrophys. Fluid Dyn., 25, 1-63.

Han, Y.J., 1984. A numerical world ocean general circulation model. Part II: A baroclinic experiment. Dyn. Atmos. Oceans, 8, 141-172.

Hellerman, S., and M. Rosenstein, 1983. Normal monthly wind stress over the world ocean with error estimates. Journal of Physical Oceanography, 13, 1093-1104.

Hidaka, K., 1940. Absolute evaluation of ocean currents in dynamic calculations. Proc. Imp. Acad. Tokyo, 16, 391-393. 
Hogg, N., 1987. A least-squares fit of the advective-diffusive equations to Levitus Atlas data. Journal of Marine Research, 45, 347-375.

Holland, W.R., 1986. Quasi-geostrophic modeling of eddy-resolved ocean circulation. In: Advanced Physical Oceanographic Numerical Modeling, J.J. O'Brien (Ed.), NATO ASI Series C: Mathematical and Physical Sciences, 186, Reidel, 203-231.

Holloway, G., 1989. Subgridscale Representation. In: Oceanic Circulation Models: Combining Data and Dynamics, D.L. Anderson and J. Willebrand (Eds.), NATO ASI Series C: Mathematical and Physical Sciences, 284, 513-583.

Jenkins, W.J., 1987. ${ }^{3} \mathrm{H}$ and ${ }^{3} \mathrm{He}$ in the Beta Triangle: Observations of Gyre Ventilation and Oxygen Utilization Rates. Journal of Physical Oceanography, 17, 763-783.

Joyce, T.M., C. Wunsch, S.D. Pierce, 1986. Synoptic Gulf Stream velocity profiles through simultaneous inversion of hydrographic and acoustic doppler data. Journal of Geophysical Research, 91, 7573-7585.

Killworth, P.D., 1986. A Bernoulli inverse method for determining the ocean circulation. Journal of Physical Oceanography, 16, 2031-2051.

Killworth, P.D., and G.R. Bigg, 1988. An intercomparison of inverse methods using an eddy-resolving general circulation model. Journal of Physical Oceanography, 18, 987-1008.

Lawson, C.L., and R.J. Hanson, 1974.Solving Least Squares Problems. PrenticeHall, Inc., Englewood Cliffs, NJ, 340 pages.

Levitus, S., 1982. Climatological Atlas of the World Ocean. NOAA Professional Paper 13, National Oceanic and Atmospheric Administration, Rockville, Maryland. 173 pages.

Louis, A.K., 1992. Medical imaging: state of the art and future development. Inverse Problems, 8, 709-738.

Lozier, M.S., W.B. Owens, and R.G. Curry, 1994. The climatology of the North Atlantic. Submitted to Prog. Oceanogr..

Marotzke, J., 1992. The role of integration time in determining a steady state through data assimilation. Journal of Physical Oceanography, 22, 1556-1567.

Marotzke, J., and C. Wunsch, 1993. Finding the steady state of a general circulation model through data assimilation: application to the North Atlantic ocean. Journal of Geophysical Research, 98, No. C11, 20149-20167. 
Martel, F., and C. Wunsch, 1993a. The North Atlantic circulation in the early 1980s- an estimate from inversion of a finite-difference model. Journal of Physical Oceanography, 23, 898-924.

Martel, F., and C. Wunsch, 1993b. Combined inversion of a finite difference model and altimetric sea surface topography. Manuscripta Geodaetica, 18, 219-226.

McDougall, T.J., 1987. Neutral Surfaces. Journal of Physical Oceanography, 17, 1950-1964.

McDougall, T.J., 1989. Streamfunction for the lateral velocity vector in a compressible ocean. Journal of Marine Research, 47, 267-284.

Memery, L., and C. Wunsch, 1990. Constraining the North Atlantic circulation with tritium data. Journal of Geophysical Research, 95, 5239-5256.

Menke, W., 1984. Geophysical Data Analysis: Discrete Inverse Theory. Academic Press, New York, 260pp.

Mercier, H., 1986. Determining the general circulation of the ocean: a non-linear inverse problem. Journal of Geophysical Research, 91, 5103-5109.

Mercier, H., 1989. A study of the time-averaged circulation in the western North Atlantic by simultaneous inversion of hydrographic and current meter data. Deep-Sea Research, 36, 297-313.

Mercier, H., 1993. An inverse model of the North Atlantic using hydrography, tracers and Lagrangian float data. Workshop on WOCE Data Assimilation, WOCE Report No. 102/93.

Mercier, H., M. Ollitrault, and P.V. Letraon, 1989. A non-linear inverse model of the mid-latitude North Atlantic Ocean-combining float and hydrographic data. CCCO/WOCE Workshop on Inversion of Ocean General Circulation Models, Royal Society, London, WCRP 23, WMO/TD No. 331, A24-26.

Middleton, J.F., and C. Garrett, 1986. A kinematic analysis of polarised eddy fields using drifter data. Journal of Geophysical Research, 91, 5094-5102.

Middleton, J.F., and J.W. Loder, 1989. Skew fluxes in polarized wave fields. Journal of Physical Oceanography, 19, 68-76.

Montgomery, R.B., 1938. Circulation in upper layers of southern North Atlantic deduced with use of isentropic analysis. Papers in Physical Oceanography and Meteorology, 6, 255pp. 
Olbers, D., 1989. Diffusion parameterizations for the climatological circulation of the North Atlantic and the southern ocean. In: Parameterization of Smallscale Processes, Muller, P. and D. Henderson (eds), 'Aha Huliko'a Proceedings, Univ. of Hawaii at Monoa, 181-204.

Olbers, D.J., M.Wenzel, and J. Willebrand, 1985. The inference of North Atlantic circulation patterns from climatological hydrographic data. Rev. Geophys., 23, 313-356.

Owens, W.B., 1979. Simulated dynamic balances for mid-ocean mesoscale eddies. Journal of Physical Oceanography, 9, 337-359.

Owens, W.B., 1991. A statistical description of the mean circulation and eddy variability in the Northwestern Atlantic using SOFAR floats. Prog. Oceanogr., 28, 257-303.

Pedlosky, J., 1987. Geophysical Fluid Dynamics. 2nd ed. Springer-Verlag, 710 pages.

Philander, S.G.H., W.J. Hurlin, and A.D. Siegel, 1987. Simulation of the seasonal cycle of Tropical Pacific Ocean. Journal of Physical Oceanography, 17, 19862002.

Plumb, R.A., 1979. Eddy waves of conserved quantities by small-amplitude waves. J. Atmos. Sci., 36, 1699-1704.

Priestley, M.B., 1981. Spectral Analysis and Time Series. Volume 1: Univariate Series. Volume 2: Multivariate Series, Prediction and Control. Academic Press, London, 890pp.

Redi, M.H., 1982. Oceanic isopycnal mixing by coordinate rotation. Journal of Physical Oceanography, 12, 1154-1158.

Reid, J., 1977. On the characteristics and circulation of the southwestern Atlantic Ocean. Journal of Physical Oceanography, 7, 62-91.

Reid, J., 1989. On the total geostrophic circulation of the South Atlantic Ocean: Flow patterns, tracers, and transports. Prog. Oceanogr., 23, 149-244.

Riley, G.A., 1951. Oxygen, phosphate and nitrate in the Atlantic Ocean. Bulletin of the Bingham Oceanographic Collection, 13:1, 126pp.

Sarmiento, J.L., 1986: On the North and Tropical Atlantic heat balance. Journal of Geophysical Research, 91, 11,677-11,689. 
Schiller, A., 1994. The mean circulation of the Atlantic Ocean north of $30^{\circ} \mathrm{S}$ determined by the adjoint method. submitted to J. Mar. Res..

Schiller, A. and J. Willebrand, 1994. A technique for the determination of surface heat and freshwater fluxes from hydrographic observations, using an approximate adjoint ocean circulation model. Submitted to J. Mar. Res..

Schlitzer, R., 1987. Renewal rates of East Atlantic deep water estimate by inversion of 14C data. Journal of Geophysical Research, 92, 2953-2961.

Schlitzer, R., 1988. Modeling the nutrient and carbon cycles of the North Atlantic. 1. Circulation, mixing coefficients, and heat fluxes. Journal of Geophysical Research, 93, 10,699-10,723.

Schlitzer, R., 1989. Model of the nutrient and carbon cycles in the North Atlantic. An application of linear programming methods. In: Oceanic Circulation Models: Combining Data and Dynamics, D.L. Anderson and J. Willebrand (Eds.), NATO ASI Series C: Mathematical and Physical Sciences, 284, 452-464.

Schlitzer, R., 1993. Determining the mean, large-scale circulation of the Atlantic with the adjoint method. Journal of Physical Oceanography, 23, 1935-1952.

Schmitt, R.W., 1994. Double diffusion in oceanography. Annu. Rev. Fluid Mech., 26, 255-285.

Schmitz, W.J., and N.G. Hogg, 1983. Exploratory observations of abyssal currents in the South Atlantic near Vema Channel. Journal of Marine Research, 41, 487-510.

Schott, F., and H. Stommel, 1978. Beta-spirals and absolute velocities in different oceans. Deep-Sea Research, 25, 961-1010.

Semtner, A.J., and R.M. Chervin, 1988. A simulation of the global ocean circulation with resolved eddies. Journal of Geophysical Research, 93, No. C12, 15502-15522.

Spall, M.A., 1992. Cooling spirals and recirculations in the subtropical gyre. Journal of Physical Oceanography, 22, 564-571.

Spall, M.A., 1994. Mechanism for low-frequency variability and salt flux in the Mediterranean salt tongue. Journal of Geophysical Research, 99, No. C5, 10121-10129.

Spall, M.A,, P.L. Richardson, and J. Price, 1993. Advection and eddy mixing in the Mediterranean Salt Tongue. Journal of Marine Research, 51, 797-818. 
Spitzer, W.S., and W.J. Jenkins, 1989. Rates of vertical mixing, gas exchange and new production: Estimates from seasonal gas cycles in the upper ocean near Bermuda. Journal of Marine Research, 47, 169-196.

Stommel, H., and F. Schott, 1977. The beta spiral and the determination of the absolute velocity field from hydrographic station data. Deep-Sea Research, 24, 325-329.

Stone, P.H., 1972. A simplified radiative-dynamical model for the static stability of rotating atmospheres. J. Atmos. Sci., 29, 405-418.

Tarbell, S., R. Meyer, N. Hogg, and W. Zenk, 1994. A moored array along the southern boundary of the Brazil Basin for the Deep Basin ExperimentReport on a joint experiment 1991-1992. Technical Report, WHOI-94-07.

Thacker, W.C., 1988. Fitting models to inadequate data by enforcing spatial and temporal smoothness. Journal of Geophysical Research, 93, No. C9, 1065510665 .

Tziperman, E., 1987. Mixing and General Circulation Dynamics: Theory and Observations. Ph.D Dissertation, MIT/WHOI Joint Program. 162p.

Tziperman, E., 1988. Calculating the Time-Mean Oceanic General Circulation and Mixing coefficients from Hydrographic data. Journal of Physical Oceanography, 18, 519-525.

Tziperman, E., and W.C. Thacker, 1989. An optimal control/adjoint equations approach to studying the oceanic general circulation. Journal of Physical Oceanography, 19, 1471-1485.

Wallace, J.M., 1978. Trajectory slopes, countergradient heat fluxes and mixing by lower stratospheric waves. J. Atmos. Sci., 35, 554-558.

Wright, R., 1969. Deep Water Movement in the Western Atlantic as determined by use of a box model. Deep-Sea Research, 16, 433-446.

Wunsch, C., 1977. Determining the General Circulation of the Ocean: A preliminary Discussion. Science, 196, 871-875.

Wunsch, C., 1978. The general circulation of the North Atlantic west of $50^{\circ} \mathrm{W}$ determined from inverse methods. Reviews of Geophysics and Space Physics, 16, 583-620.

Wunsch, C., 1980. On using satellite altimetry to determine the general circulation of the oceans with application to geoid improvement. Reviews of Geophysics and Space Physics, 18, 725-745. 
Wunsch, C., 1984. An eclectic Atlantic Ocean circulation model. Part 1: The meridional flux of heat. Journal of Physical Oceanography, 14, 1712-1733.

Wunsch, C., 1988a. Eclectic modeling of the North Atlantic, Part 2: Transient tracers and the ventilation of the eastern basin thermocline. Phil. Tran. Roy. Soc., A325, 201-236.

Wunsch, C., 1988b. Transient tracers as a problem in control theory. Journal of Geophysical Research, 93, 8099-8110.

Wunsch, C., 1989. Tracer Inverse Problems. in Oceanic Circulation Models: Combining Data and Dynamics, D.L. Anderson and J. Willebrand, (Eds.), NATO ASI Series C: Mathematical and Physical Sciences, 284, 1-14.

Wunsch, C., 1994. Dynamically consistent hydrography and absolute velocity in the eastern North Atlantic ocean. Journal of Geophysical Research, 99, No. C7, 14071-14090.

Wunsch. C., and E.M. Gaposchkin, 1980. On using satellite altimetry to determine the general circulation of the oceans with application to geoid improvement. Journal of Geophysical Research, 18, 725-745.

Wunsch, C., and B. Grant, 1982. Towards the general circulation of the North Atlantic Ocean. Prog. in Oceanogr., 11, 1-59.

Wüst, G., 1935. Schichtung und Zirkulation des Atlantischen Ozeans. Die Stratosphäre. In Wissenschaftliche Ergebnisse der Deutschen Atlantischen Expedition auf dem Forschungs-und Vermessungsschiff "Meteor" 1925-1927, 6: 1st Part, 2, $180 p p$.

Zhang, H., and N.G. Hogg, 1992. Circulation and water mass balance in the Brazil Basin. Journal of Marine Research, 50, 385-420. 